\title{
COLLOIDAL PROBE FORCE SPECTROSCOPY
}

\section{A mechanical pathway from Membrane Fusion through out Nuclear Transport Mechanism}

\author{
Dissertation at \\ Georg-August University Göttingen \\ for the award of the degree \\ Doctor rerum naturalium \\ submitted by \\ Jörn Dietz
}

Accomplished within the doctoral program

Physics of Biological and Complex Systems

of the Georg-August University School of Science (GAUSS)

Göttingen 2021 

This doctoral dissertation was carried out in the working group of Prof. Dr. Andreas Janshoff at the Institute of Physical Chemistry at the Georg-August University Göttingen and Advisory services of GGNB.

\section{Thesis Advisory Commitee:}

Prof. Dr. Andreas Janshoff

Institute for Physical Chemistry, Georg-August-Universität Göttingen

Prof. Dr. Tim Salditt

Institute for X-Ray Physics, Georg-August-Universität Göttingen

Prof Dr. Dirk Görlich

Cellular Logistics, Max Planck Institute for Biophysical Chemistry Göttingen

\section{Members of the Examination Board:}

Prof. Dr. Andreas Janshoff

Institute for Physical Chemistry, Georg-August-Universität Göttingen

Prof. Dr. Tim Salditt

Institute for X-Ray Physics, Georg-August-Universität Göttingen

Prof Dr. Dirk Görlich

Cellular Logistics, Max Planck Institute for Biophysical Chemistry Göttingen

\section{Further Members of the Examination Board:}

Prof. Dr. Peter J. Walla

Institute of Physical and Theoretical Chemistry TU Braunschweig

Prof. Dr. Michael Meinecke

University Medical Center Göttingen Dept. of Cellular Biochemistry

Prof. Dr. Marcus Müller

Institute for Theoretical Physics Georg-August-Universität Göttingen

Date of oral examination: 03.05.2021 



\section{Declaration regarding $\S 4$ section 6 RerNat-O:}

I, Jörn Dietz, hereby declare that my doctoral thesis entitled COLLOIDAL PROBE FORCE SPECTROSCOPY - A mechanical pathway from Membrane Fusion through out Nuclear Transport Mechanism has been written independently and with no other sources nor aids than quoted. 



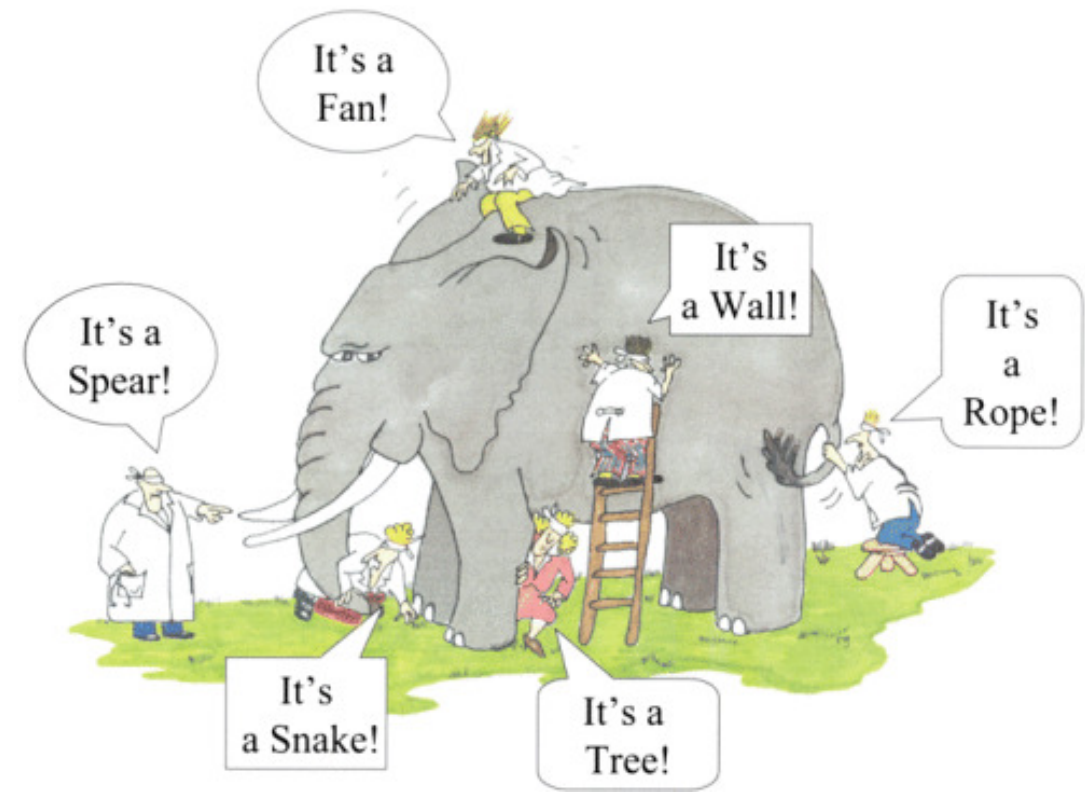

Different scientists touch different parts of the nature:

...truth is always stated from different perspectives. ${ }^{1}$

\footnotetext{
${ }^{1}$ Prisoners in Plato's Cave \& the Blind Man Metaphor: https:/ /www.complexity.org.uk/
} 



\begin{abstract}
This thesis demonstrates how colloidal probe force spectroscopy, in connection with reconstituted biological model systems and theoretical models, allows us to investigate and understand the behavior of a wide variety of physical-biological systems like membrane fusion of vesicles or cells throughout nuclear transport events. We explore the use of colloidal particles of various sizes, which, coupled to different springs, serve to measure forces and break down physical quantities into distributions. We show how these colloidal probes $(\mathrm{CP})$ can examine distinct biology areas and address different time and size scales. This extends from a global cellular level over vesicular orders of magnitude to individual molecular transport sizes. We applied force spectroscopy techniques based on colloidal particles to study mechanical behavior in three different model systems: transport through nuclear pore complexes, myogenesis of myoblast cells, and SNARE-mediated membrane fusion.
\end{abstract}

In chapter 4, the nuclear pore complex-inspired system shows how one can visualize mechanically small and hypothetical porous pathways for biomolecules hidden from optical observation. This approach implies being appropriate for nuclear pore complexes and hydrogels in general, membrane-less organelles, or liquid-liquid-phaseseparated (LLPS) systems overall.

In chapter 5, it shows myoblast cellular fusion and the cellular membrane's mechanical properties in different states of myogenesis. To study it as a small functional system with the influence of the myomerger-ectodomain in detail, we isolated it from the biochemical signals sent by parts of the cell by employing model membrane system components on colloidal particles. It allowed us to distinguish the intrinsic physics of membrane fusion from this biological system apart from the cell's regulatory processes.

Lastly, in chapter 6, we employ colloidal probes attached to distinct spring-potentials to measure nanometer-scale interactions of micrometer-sized colloids (1-15 um) to facilitate areas of weak, specific protein-bilayer interactions in the context of SNAREmediated membrane fusion. Performed in the presence and absence of bilayer-anchored f-Synaptotagmin-1 as Calcium ions were present in solution. We perform these experiments based on Atomic Force Microscopy (AFM), Optical Tweezers (OT), and Holographic Video Particle Tracking (HVPT) as they enable us to first discriminate intuitively intermediate states from step heights and corresponding lifetimes of the fusion pathway. Based on this, we resolve them by colloidal particle motion fluctuation with even greater detail. AFM and OT have been used to measure weak, kT scale inter- 
actions between membrane coated colloids and target bilayer surface as we measure separation-dependent interactions between protein-bilayer binding partners. Our ultimate results demonstrate the use of optical microscopy methods and particle tracking to quantify the connections between potentials of mean force and the crucial behavior of specific protein-bilayer interactions. Our knowledge from AFM and OT allowed us to separate these from fusion events.

Throughout these different systems, colloidal probes connected with optical microscopy, optical tweezers, or atomic force microscopy provide a resourceful means to study biological systems on various length scales and visualize dynamic biological processes with high throughput and minimal reliance on fluorescent labels. Colloidal probes have many potentials to provide further insights into the details of Membrane Fusion of vesicles or cells and Nuclear Transport. 


\section{TABLE OF CONTENTS}

$1 \quad$ Introduction into Systems studied with Colloidal Probes 2

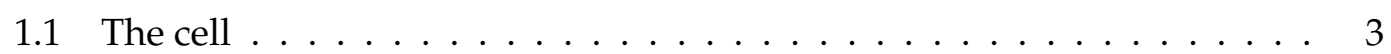

1.2 Artificial membranes and membrane fusion . . . . . . . . . . . . . 4

1.3 The cell nucleus and nuclear pore complexes . . . . . . . . . . . . 7

Reference ............................ . . . 9

2 Introduction of Colloidal Particles as Force and Displacement Probing Sen-

Sor $\quad 14$

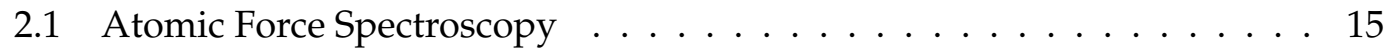

2.2 Mechanical evaluation of indentation experiments . . . . . . . . . . . . . 19

2.2.1 Hertzian contact mechanics: indentation of elastic material [65]] . 19

2.2.2 Tension measurements through membrane tether formation . . . 21

$2.2 .3 \quad$ Linear viscoelasticity [65] . . . . . . . . . . . . . . . . 25

2.2 .4 Poroelastic indentation [65] . . . . . . . . . . . . . . . . 26

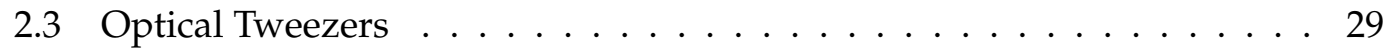

$2.4 \quad$ Free and confined diffusion of colloidal probes in solution . . . . . . . . 31

$2.4 .1 \quad$ Holographic video particle tracking . . . . . . . . . . . . 33

2.4 .2 Tethered particle motion and analysis of video particle tracking

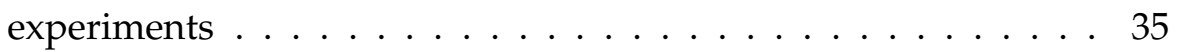

$2.4 .3 \quad$ Introducing principal component analysis and changepoint segmentation . . . . . . . . . . . . . . 40

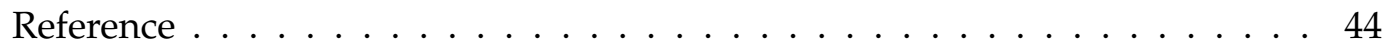

3 Biophysical Materials and Techniques

3.1 Buffers, chemicals and detergents . . . . . . . . . . . . . . . 55

3.1 .1 Buffers . . . . . . . . . . . . . . . . . . . . 55

3.1 .2 Lipids . . . . . . . . . . . . . . . . . . 55

3.1 .3 Proteine . . . . . . . . . . . . . . . . . . 57

3.1 .4 Detergents . . . . . . . . . . . . . . . . . 60

3.1 .5 Fluorescent dyes . . . . . . . . . . . . . . . . 60 
3.2 Materials . . . . . . . . . . . . . . . . . . 61

3.2 .1 Unilamellar vesicles . . . . . . . . . . . . . . . . . 62

3.2 .2 Glass substrate . . . . . . . . . . . . . . . . . 63

3.2 .3 Silica beads . . . . . . . . . . . . . . . . . . . 63

3.2 .4 Polydimethylsiloxan . . . . . . . . . . . . . . . . . . 63

3.3 Artificial bilayers and protein reconsitution $\ldots \ldots \ldots \ldots \ldots 65$

$3.3 .1 \quad$ Preparation of lipid films $\ldots \ldots \ldots \ldots \ldots \ldots$

3.4 Reconstitution of SNARE proteins into liposomes $\ldots \ldots \ldots$. . . . . 65

$3.4 .1 \quad$ Preparation of small proteoliposomes $\ldots \ldots \ldots \ldots$. . . . . . 65

3.4 .2 Reconstitution of syt-1 into liposomes $\ldots \ldots \ldots \ldots$

3.4 .3 Co-reconstitution of syt-1 and syb 2 into liposomes . . . . . . . 66

3.4 .4 Membrane-coated glass spheres $\ldots \ldots \ldots \ldots$. . . . . . . 67

3.5 Analytical fluorescence microscopy $\ldots \ldots \ldots \ldots \ldots$. . . . . . . . 68

3.5 .1 Incident light fluorescence microscopy _... . . . . . . . . . 69

3.5 .2 Confocal fluorescence microscopy $\ldots \ldots \ldots \ldots$

3.5 .3 Fluorescence recovery after photobleaching. . . . . . . . . . 70

3.5.4 Preparation of solid support membranes for influence of $\mathrm{PI}(4,5) \mathrm{P}_{2}$ on SNARE mediated membrane fusion $\ldots \ldots \ldots \ldots 72$

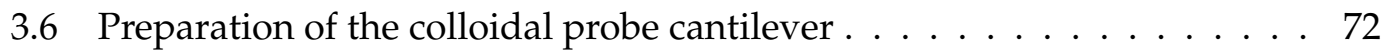

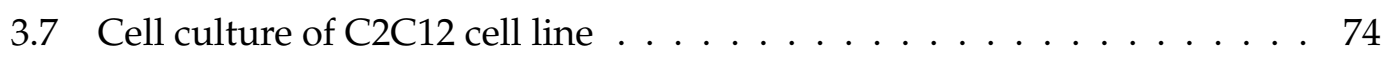

Reference . . . . . . . . . . . . . . . . . . . . . . . . . 77

4 Mechanical Characterization of Phase Separated Droplets and its Implication on Nuclear Transport $\quad 81$

4.1 Mechanical response upon indentation. . . . . . . . . . . . . . . 89

4.2 Poroelastic relaxation response and influence of nuclear transport recep-

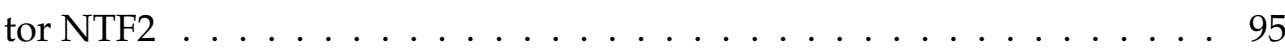

4.3 Destruction of the hydrogel network upon dissolution of underlying

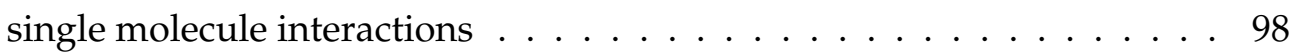

4.4 Conclusion . . . . . . . . . . . . . . . . . . . . . . . . 103

Reference . . . . . . . . . . . . . . . . . . . . . . . . . . 108

5 Cellular Mechanics during Myogenisis of Myoblast Cells 116

5.1 Atomic Force Microscopy indentation and retraction experiments . . . 120

5.2 Conversion into model systems based on colloidal probes. . . . . . . 125

5.3 Conclusion . . . . . . . . . . . . . . . . . . . . . . . . . 128

Reference . . . . . . . . . . . . . . . . . . . . 128

6 SNARE mediated Membrane Fusion and Translation into a Colloidal System 132 
6.1 Discrimination of fusion by identifying interactions and intermediates of membrane fusion mediated by SNAREs . . . . . . . . . . . . 136

$6.1 .1 \quad$ Atomic Force Spectroscopy measurements . . . . . . . . . . . 136

$6.1 .2 \quad$ Optical Tweezer measurements . . . . . . . . . . . . . . . . . . . . 138

6.1 .3 Holographic Video Particle Tracking measurements . . . . . . . . 146

6.2 Influence of $\mathrm{PI}(4,5) \mathrm{P}_{2}$ for calcium-mediated and synaptotagmin-triggered exocytosis . . . . . . . . . . . . . . . . . . . 156

6.2.1 Atomic Force Spectroscopy - membrane interaction and forces of synaptotagmin . . . . . . . . . . . . . . . 157

6.2.2 Interaction landscapes of synaptotagmin from tethered particle motion experiments and its implications on SNARE mediated membrane fusion . . . . . . . . . . . . . . . . . . . . . 160

6.3 Conclusion . . . . . . . . . . . . . . . . . . . . . . . . . . . 175

Reference . . . . . . . . . . . . . . . . . . . . . . . 177

\begin{tabular}{ll}
\hline Appendix & 183
\end{tabular}

\begin{tabular}{ll}
\hline List of Figures & 184
\end{tabular}

\begin{tabular}{ll}
\hline Abbreviations & 189
\end{tabular}

\begin{tabular}{ll}
\hline Acknowledgement & 196
\end{tabular} 


\section{Outline of the Thesis}

After giving a general introduction to three biological systems from large to small length scales in chapter 1, chapter 2 will provide a general introduction to the methodology. This focuses on colloidal particles, which are used to study different time and size scales. Atomic force microscopy, optical tweezers, and video particle tracking were used.

Chapter 3 presents all the materials and evaluation methods used, either developed in-house or made available by collaboration partners.

Chapter 4 examines phase-separated hydrogels' mechanical properties, which are used as a model system for the transport of molecules through nuclear pores.

Chapter 5 examines, on the one hand, the mechanical properties by AFM of the cell membrane when myoblast cells differentiating and fusing and attempts to investigate these in a $2 \mathrm{D}$ particle assay for fusion efficiency.

Chapter 6 ultimately combines three methodological approaches and shows how these can be used to investigate the fusion of artificial membranes in the context of SNAREmediated and synaptotagmin-supported membrane fusion.

In summary, the last three chapters show how we explored complex biological systems with colloidal probes addressing different time and length scales. 


\section{CHAPTER 1}

\section{INTRODUCTION INTO SYSTEMS STUDIED WITH COLLOIDAL PROBES}

Complex biological systems are an interdisciplinary field that studies the nature of a sytem like it is shown in Figure 1.1 starting from single motifs to complex interplays. This conceptual framework develops and uses methods and aspects applicable to various areas, such as biology, chemistry, and physics [3, 21]. We understand a system and its mechanism or behavior by imagine it as a whole and breaking it apart into pieces that we can understand or describe; observing its interaction between itself and its embedding environment, and deriving complex and often subtle trajectories of mostly dynamic behavior that sometimes are stable and reinforcing, or at various boundary conditions can become unstable.

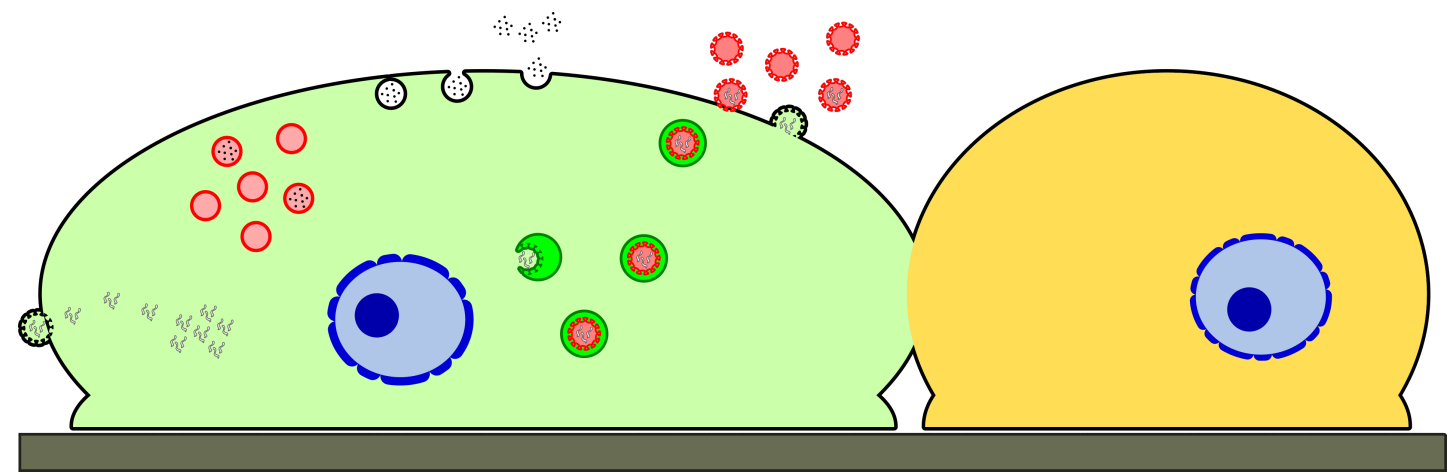

Figure 1.1: Schematic illustration of a complex interplay of two system units like cells fusing through merging their outer bilayers as processes like exo- and endocytosis, viral infection, and nuclear transport into the cell nucleus are present. 


\subsection{The cell}

A cell is a complex system consisting of a fluid envelope, the cell membrane, which encloses the various organelles like the cell nucleus, the cytoskeleton, and the cytosol [1]. In addition to the plasma membrane, membranes can be found in the cell wherever different functional compartments are delimited. Whether it is a compartment within the cytosol or the entire cell interior's delimitation to the outside, membranes consist of various lipids and steroids mostly arranged in a double layer. Embedded in this liquid-crystalline matrix are membrane proteins, which, as functional units, guarantee transport across the dielectric barrier, acting as receptors or have an enzymatic effect [10, 31]. Its composition depends on the type of cell and the particular function of the membrane. The membrane is responsible for delimitation, selective transport of molecules, and signal transmission across the membrane [6].

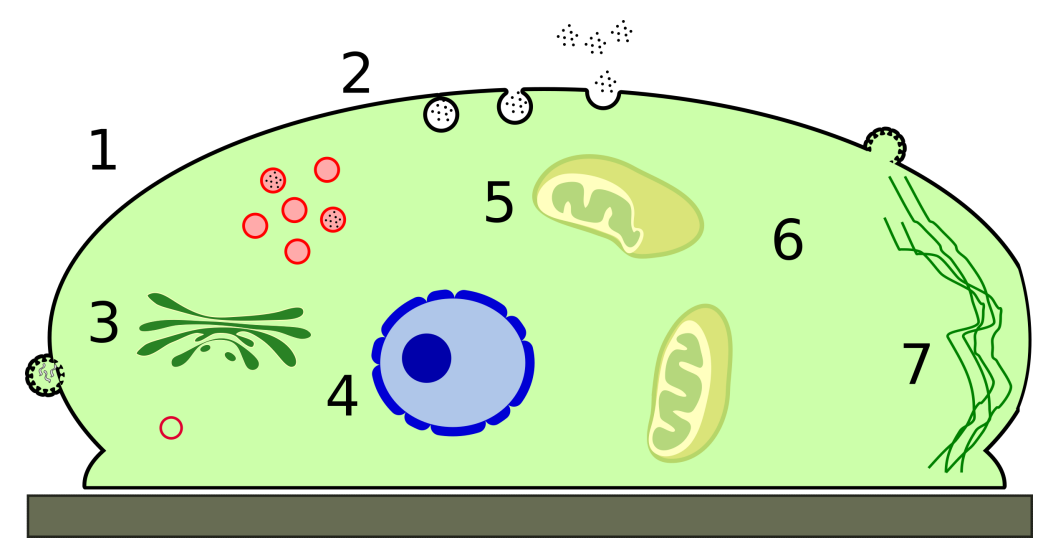

Figure 1.2: Schematic illustration of an eukaryotic cell and its organelles. 1: plasma membrane. 2: secretory vesicles. 3: golgi apparatus. 4: nucleus, surrounded by a nuclear envelope. 5: mitochondria. 6: cytoplasma. 7: actin filaments.

Most importantly, in animal cells, the cell membrane, together with the cytoskeleton, is responsible for its mechanical properties and shape [9]. Wounding drives individual sections and cell layers to change and constraints them to the adaption of shape and their mechanical properties and vice versa. Adaptation can be accompanied by cellular membrane fusion, for example, in the development or regeneration of skeletal muscles [33]. This unique tissue is made up of multinucleated muscle fiber bundles. Each myofiber is the product of the fusion of hundreds or thousands of mononucleated muscle cells known as myoblast cells.

Regardless of length scale, membrane fusion is typically a multistep process that, upon completion, unites two lipid membranes and the volumes they envelop and is vital for multiple biological processes, including the development of syncytial tissues, viral infection, and exocytosis [8, 12, 36]. 


\subsection{Artificial membranes and membrane fusion}

The cellular plasma membrane owns specific properties to carry out its function. A membrane is a two-dimensional, fluid-structure made up of amphiphilic molecules such as lipids, shown in Figure 1.3. The phospholipids' amphiphilic character, which is found in natural membranes, results from a polar or charged, phosphate-bearing head group that is connected to two non-polar hydrocarbon chains via a glycerine [1]. When amphiphilic molecules are placed in water, they spontaneously self-assemble into aggregates like micelles, vesicles, and bilayers. This aggregation is driven by the hydrophobic effect, which describes non-polar molecules' behavior in contact with water. The segregation of non-polar molecules and water leads to spontaneous selfassembly of various aggregates [38]. Regarding lipids, the shape of these aggregates depends on the geometry of the individual molecules. Because of their cylindrical shape, and the self-organization in polar media, phospholipids tend to form a $4-5 \mathrm{~nm}$ thick lipid bilayer as illustrated in Figure 1.3 (D) [19, 25].

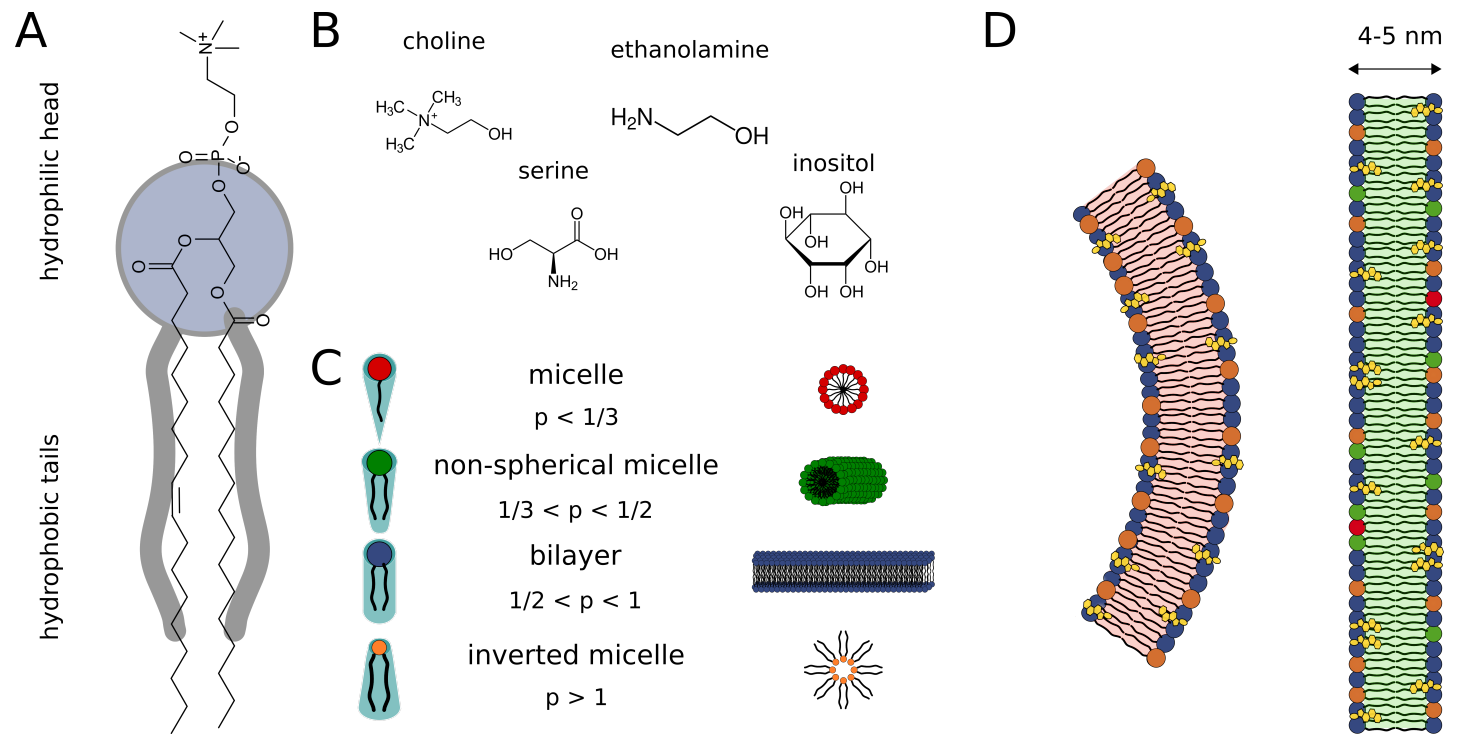

Figure 1.3: Schematic illustration of common phospholipid structures and geometries. A: chemical structure of a phospholipid, highlighted are head and tail group to emphasize amphiphilic character. B: chemical structure of most common alcohols building the hydrophilic head of a lipid. C: packing parameter $p$ predicts the structure of the aggregates formed from lipids placed in aqueous solution. D: curve and planar artificial membrane.

In addition to separating the cell's inner space's compartment from the outside, the plasma membrane is a vital in cell-to-cell contact and intracellular signal initiation. Various cellular proteins interact and affect critical cellular processes with the membrane surfaces. Proteins can guide these interactions towards a particular lipid component within a membrane [7]. Phosphatidylinositol-4,5-bisphosphate $\left(\mathrm{PI}(4,5) \mathrm{P}_{2}\right)$, for instance, 
is the most abundant phospho-inositide in the inner leaflet of mammalian plasma membranes with about $1 \%$ of total lipids, and its list of cellular functions is steadily rising [4, 13, 23]. It plays a crucial role in vesicle exocytosis within neurons, in addition to a large number of protein- $\mathrm{PI}(4,5) \mathrm{P}_{2}$-membrane interactions. Here because of a transient $\mathrm{Ca}^{2+}$-influx through voltage-gated $\mathrm{Ca}^{2+}$-channels evoked by an incoming action potential, synaptic vesicles fuse with the presynaptic membrane [20]. Upon neuronal exocytosis, synaptic vesicles release neurotransmitters into the synaptic cleft for signal transmission.

Transmission in neurons or hormone secretion in neuroendocrine cells are fundamental biological processes, which are mediated by SNARE-(soluble N-ethyl-maleimidesensitive-factor attachment receptor) membrane fusion. This process's exact mechanism, particularly how a transient rise of the intracellular $\mathrm{Ca}^{2+}$-concentration triggers, clamps, or even accelerates fusion, is still a matter of interest [17, 18, 40].

Exocytosis and the fusion of two membranes are processes that are not spontaneous. Instead, they are caused by an interplay of many different proteins, such as Synaptotagmin, Complexin, Munc18, and Munc13, regulated and take place in the so-called active zones [37]. These proteins provide the energy required for fusion and enable comprehensive control of the fusion processes that are taking place. The essential proteins occurring in eukaryotic cells belong to the so-called SNARE family. SNARE proteins mediate the synaptic vesicles' fusion with the axon's presynaptic membrane. The stable core complex consists of the three subunits Synaptobrevin-1-116, Syntaxin-1A and SNAP-25, each of which forms four parallel $\alpha$-helices like a zipper using a heptad repeat sequence. This core complex's interior is formed from 15 layers of hydrophobic amino acids perpendicular to the twist axis. The coiled-coil motif shown in Figure 1.4 of $v$ - and $t$-SNARE proteins, which are located on the vesicle surface and the target membrane, mediates the approach of the vesicles to the membrane surface and thus supports the fusion during exocytosis [16, 41].

A sensitive $\mathrm{Ca}^{2+}$-sensor is needed to ensure this highly controlled synchronous process by $\mathrm{Ca}^{2+}$-influx. One of the critical candidates sensing $\mathrm{Ca}^{2+}$ in neuronal exocytosis was determined to be Synaptotagmin-1 (syt-1) as shown in Figure 1.5. But the molecular mechanism by which syt- 1 activates $\mathrm{Ca}^{2+}$-dependent vesicle fusion remains uncertain, even though a large number of studies have been published on this subject [24, 34]. A model was developed by Honigmann et al. to explain how syt- 1 can bridge two separate membranes, taking account of the syx- 1 microdomains. They propose that $\mathrm{PI}(4,5) \mathrm{P}_{2}$ explicitly links the syt- 1 and syx-1A C2B domains in a $\mathrm{Ca}^{2+}$-independent 


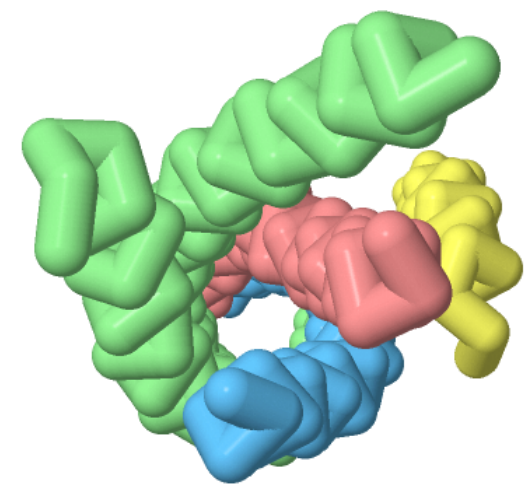

(a) SNAREs and complexin

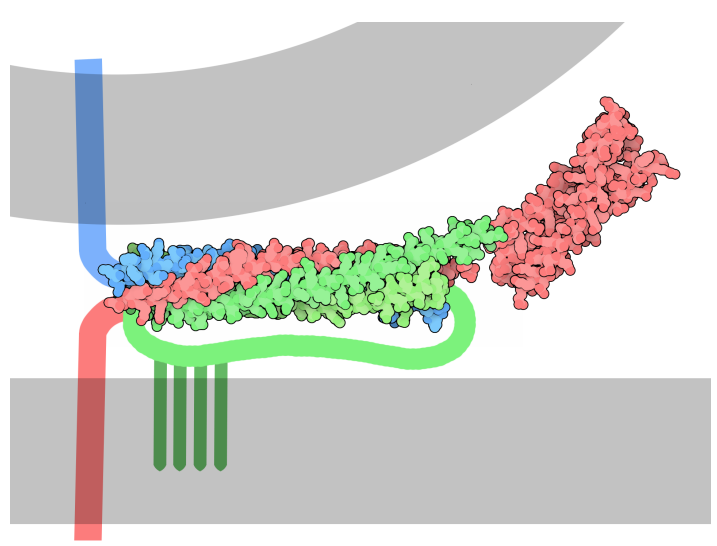

(b) v-, t-SNARE coiled coil complex

Figure 1.4: Illustration of formed four-helix coiled coil SNARE-complexes: syntaxin (red), synaptobrevin (blue), SNAP-25 (green), complexin (yellow).

manner based on the PI $(4,5) \mathrm{P}_{2}$ polyanionic headgroup and the syx-1A (260-KARRKK265) and C2B syx-1A polybase sequences (324-KKKK-327). To tether the two opposite membranes together, $\mathrm{Ca}^{2+}$ is then required [14, 26, 29].

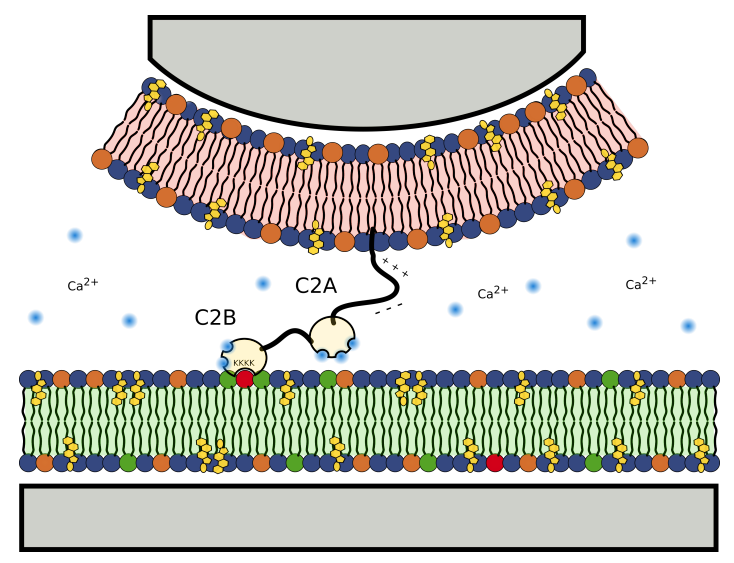

Figure 1.5: Schematic illustration of a proposed model of tethering induced by the C2-domains of syt-1. $\mathrm{Ca}^{2+}$ (blue)-independent binding of the polybasic sequence of synaptotagmins $\mathrm{C} 2 \mathrm{~B}$ domain to microdomains enriched with $\mathrm{PI}(4,5) \mathrm{P}_{2}$ (red). $\mathrm{Ca}^{2+}$ dependent tethering by synaptotagmins $\mathrm{C} 2 \mathrm{~A}$ and $\mathrm{C} 2 \mathrm{~B}$ domains, in which the $\mathrm{C} 2 \mathrm{~B}$ domain interacts with the plasma membrane.

Seventeen isoforms of the family of synaptotagmin (syt) proteins have been discovered. However, we will characterize only one isoform in detail as this study's focus was on syt- 1 and its effect on in vitro SNARE-mediated membrane fusion. Syt- 1 is a protein that binds to $\mathrm{Ca}^{2+}$, specifically located in the membrane of synaptic vesicles and secretory granules in endocrine cells. It consists of the short unstructured intravesicular N- 
terminal part followed by the $\alpha$-helical transmembrane domain (TMD) and the cytosolic region as its central part. The two $\mathrm{Ca}^{2+}$-binding domains, $\mathrm{C} 2 \mathrm{~A}$ and $\mathrm{C} 2 \mathrm{~B}$, which bind 3 and $2 \mathrm{Ca}^{2+}$ ions respectively, are housed in the cytosolic portion of syt- 1 . The aspartate residues that coordinate the $\mathrm{Ca}^{2+}$-ions are located at the distal tips of the $\mathrm{C} 2$-domains in flexible loops [5, 35].

As a requirement for $\mathrm{Ca}^{2+}$-dependent tethering, following previous findings of the high affinity of the $\mathrm{C} 2$-domains to acidic phospholipids in their $\mathrm{Ca}^{2+}$-bound state, the vesicle membrane needs at least $5 \%$ of the anionic lipid phosphatidylserine (PS) [11, 43].

In general, due to the high complexity of biological systems, it makes sense to elucidate fundamental mechanisms in detail using model systems. One can precisely control the external conditions, and the components involved in the model system and observed processes are easier to understand.

We will use colloidal probes as a template to gain insights into this complex field of protein-mediated membrane-membrane interactions up to SNARE-mediated membrane fusion, and membrane mechanics as anticipated in Figure 6.1 and 6.2. This application of artificial lipid membranes, employing solid-supported membranes (SSM), will build a comprehensive model system to mimic plasma membrane fusion processes in living organisms.

\subsection{The cell nucleus and nuclear pore complexes}

The nucleus is a highly specialized organelle which acts as the data processing and administrative center of the cell. This organelle has two primary functions: it stores the genetic material or DNA of the cell and controls the cell's operations, including growth, intermediate metabolism, synthesis of proteins, and reproduction. Only the cells known as eukaryotes have a nucleus. They have a double lipid bilayer called the nuclear envelope (NE) that engulfs their genetic material and determines the heart, in contrast to prokaryotes [1]. With this structure, shown in Figure 1.6, there are several advantages: it protects the genome from external or internal causes of damage due to shear stress or large moving objects, e.g., cytoskeletons and organelles, and mainly acts as a barrier defense against pathogens such as viruses [30].

Despite these benefits, the difficulty of overcoming the NE-barrier is faced by eukaryotes. For instance, the cell must ensure that mature mRNA is exported into the cytoplasm, but at the same time allow transcription factors to reach the nucleus to initiate transcription[27]. Nuclear pore complexes (NPCs) that pierce the NE in large 


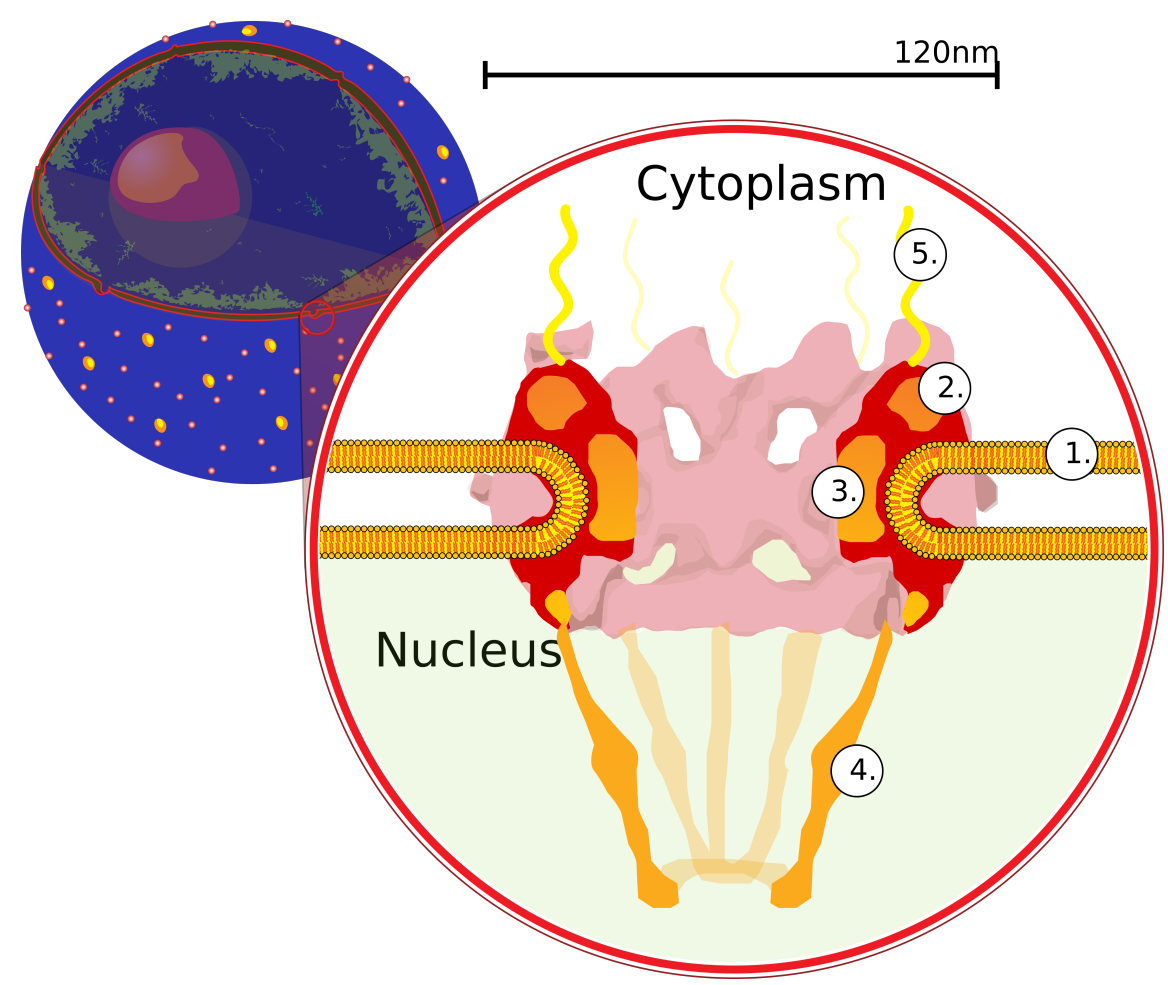

Figure 1.6: Schematic illustration of the nuclear pore complex structure: 1 . outer and inner membrane of the nuclear envelope. 2. outer ring and linker nups. 3. inner ring and central FG-Nups 4. nuclear FG-Nups and basket 5. cytoplasmic FG-Nups and filaments. Figure adapted from wikimedia under the creative commons attribution international license.

numbers and constitute the remaining gateway between the nucleus and the cytoplasm achieve this bidirectional traffic [42].

Around 30 different proteins, called nucleoporins or Nups, are found in each NPC, present in several copies per NPC [15]. The size of the NPC varies between species, but it shares a general 8-fold symmetry pattern [32]. Transmembrane Nups act as anchor points to the NE and constitute the NPCs' central structure of 40-90 nm height that surrounds the aqueous channel, together with linker Nups and inner ring Nups. The pore is lined at its periphery with the central channel Nups and has an inner diameter of 40-75 nm [2]. All central channel Nups contain intrinsically disordered domains rich in so-called FG repeats or FG-Nups, rich in Phenylalanine (F) and Glycine (G), which are presumed to contribute collectively via the NPC to a selective barrier controlling cargo translocation [28].

A sequence of protein-protein interactions accomplishes successful transport between the nucleus and cytoplasm of cargo molecules larger than $5 \mathrm{~nm}$ in size arranged by Karyopherin (Kaps) that exhibit exclusive passage through NPCs [39]. 
Nucleoporins play a role in the assembly and maintenance of the nuclear pore complex. Specifically, Nup98 and Nup96 are involved in bidirectional transport around the NPC. Interestingly, Nup98 binds to complexes of HIV-1 capsid-nucleocapsid (HIV-1 CA-NC) and can facilitate in vitro incorporation of the virus into the host nucleus [22].

\section{Reference}

[1] Bruce Alberts, Alexander Johnson, Julian Lewis, Peter Walter, Martin Raff, and Keith Roberts. Molecular Biology of the Cell 4th Edition: International Student Edition. Routledge, 2002. ISBN 9780815332886. Google-Books-ID: ozigkQEACAAJ.

[2] Aizhan Bestembayeva, Armin Kramer, Aksana A. Labokha, Dino Osmanović, Ivan Liashkovich, Elena V. Orlova, Ian J. Ford, Guillaume Charras, Ariberto Fassati, and Bart W. Hoogenboom. Nanoscale stiffness topography reveals structure and mechanics of the transport barrier in intact nuclear pore complexes. Nature Nanotechnology, 2015. 10(1): 60-64. doi:10.1038/nnano.2014.262.

[3] Enrico Bibbona. Stochastic chemical kinetics. theory and (mostly) systems biological applications, p. erdi, g. lente. springer (2014). Biosystems, 2014. 126: 85-86. doi:10.1016/j.biosystems.2014.10.004.

[4] Luís Borges-Araújo and Fabio Fernandes. Structure and lateral organization of phosphatidylinositol 4,5-bisphosphate. Molecules, 2020. 25(17): 12-14. doi: $10.3390 /$ molecules 25173885 .

[5] Nils Brose, Alexander G. Petrenko, Thomas C. Sudhof, and Reinhard Jahn. Synaptotagmin: A calcium sensor on the synaptic vesicle surface. Science, 1992. 256(5059): 1021-1025. doi:10.1126/science.1589771.

[6] Xiaolin Cheng and Jeremy C. Smith. Biological Membrane Organization and Cellular Signaling. Chemical Reviews, 2019. 119(9): 5849-5880.

[7] Valentina Corradi, Eduardo Mendez-Villuendas, Helgi I. Ingólfsson, Ruo-Xu Gu, Iwona Siuda, Manuel N. Melo, Anastassiia Moussatova, Lucien J. DeGagné, Besian I. Sejdiu, Gurpreet Singh, Tsjerk A. Wassenaar, Karelia Delgado Magnero, Siewert J. Marrink, and D. Peter Tieleman. Lipid-Protein Interactions Are Unique Fingerprints for Membrane Proteins. ACS Central Science, 2018. 4(6): 709-717. doi:10.1021/acscentsci.8b00143.

[8] Su Deng, Mafalda Azevedo, and Mary Baylies. Acting on identity: Myoblast fusion 
and the formation of the syncytial muscle fiber. Seminars in cell $\mathcal{E}$ developmental biology, 2017. 72. doi:10.1016/j.semcdb.2017.10.033.

[9] Daniel a Fletcher and R Dyche Mullins. Cell mechanisms and cytoskeleton. Nature, 2010. 463(7280): 485-492. doi:10.1038/nature08908.Cell.

[10] David E. Green and Robert F. Brucker. The Molecular Principles of Biological Membrane Construction and Function. BioScience, 1972. 22(1): 13-19.

[11] Clémence Gruget, Oscar Bello, Jeff Coleman, Shyam S. Krishnakumar, Eric Perez, James E. Rothman, Frederic Pincet, and Stephen H. Donaldson. Synaptotagmin-1 membrane binding is driven by the $\mathrm{C} 2 \mathrm{~B}$ domain and assisted cooperatively by the C2A domain. Scientific Reports, 2020. 10(1): 1-10. doi:10.1038/s41598-020-74923-y.

[12] Stephen C. Harrison. Viral membrane fusion. Virology, 2015. 479-480: 498-507. doi:10.1016/j.virol.2015.03.043.

[13] Eamonn J. Dickson Hille and Bertil. Understanding phosphoinositides: rare, dynamic, and essential membrane phospholipids Eamonn. Physiology E behavior, 2017. 176(3): 139-148.

[14] Alf Honigmann, Geert van den Bogaart, Emilio Iraheta, Herre Jelger Risselada, Dragomir Milovanovic, Veronika Mueller, Stefan Müllar, Ulf Diederichsen, Dirk Fasshauer, Helmut Grubmüller, Stefan W. Hell, Christian Eggeling, Karin Kühnel, and Reinhard Jahn. Phosphatidylinositol 4,5-bisphosphate clusters act as molecular beacons for vesicle recruitment. Nature structural \& Molecular Biology, 2013. 20(6): 679-686. doi:10.1038/nsmb.2570.Phosphatidylinositol.

[15] Arkaitz Ibarra and Martin W. Hetzer. Nuclear pore proteins and the control of genome functions. Genes and Development, 2015. 29(4): 337-349. doi:10.1101/gad. 256495.114.

[16] Reinhard Jahn and Richard H. Scheller. SNAREs — engines for membrane fusion. Nature Reviews Molecular Cell Biology, 2006. 7(9): 631-643. doi:10.1038/nrm2002.

[17] Volker Kiessling, Alex J. B. Kreutzberger, Binyong Liang, Sarah B. Nyenhuis, Patrick Seelheim, J. David Castle, David S. Cafiso, and Lukas K. Tamm. A molecular mechanism for calcium-mediated synaptotagmin-triggered exocytosis. Nature Structural \& Molecular Biology, 2018. 25(5): 139-148. doi:https: //doi.org/10.1038/s41594-018-0130-9.

[18] Alex J.B. Kreutzberger, Volker Kiessling, Binyong Liang, Patrick Seelheim, Shrutee 
Jakhanwal, Reinhard Jahn, J. David Castle, and Lukas K. Tamm. Reconstitution of calcium-mediated exocytosis of dense-core vesicles. Science Advances, 2017. 3(7).

[19] Barbara A. Lewis and Donald M. Engelman. Lipid bilayer thickness varies linearly with acyl chain length in fluid phosphatidylcholine vesicles. Journal of Molecular Biology, 1983. 166(2): 211-217.

[20] Thomas F.J. Martin. Role of pi(4,5)p2 in vesicle exocytosis and membrane fusion. Subcellular Biochemistry, 2015. 59: 111-130.

[21] Pedro J. Miranda and Giuliano La Guardia. On a relational theory of biological systems: a natural model for complex biological behavior. aRxiv, 2017.

[22] Anne Monette, Nelly Panté, and Andrew J. Mouland. HIV-1 remodels the nuclear pore complex. Journal of Cell Biology, 2011. 193(4): 619-631. doi:10.1083/jcb. 201008064.

[23] Frauke Mücksch, Mevlut Citir, Christian Lüchtenborg, Bärbel Glass, Alexis Traynor-Kaplan, Carsten Schultz, Britta Brügger, and Hans Georg Kräusslich. Quantification of phosphoinositides reveals strong enrichment of PIP2 in HIV-1 compared to producer cell membranes. Scientific Reports, 2019. 9(1): 1-13. doi: 10.1038/s41598-019-53939-z.

[24] Sarah B. Nyenhuis, Anusa Thapa, and David S. Cafiso. Phosphatidylinositol 4,5 bisphosphate controls the cis and trans interactions of synaptotagmin 1. Biophysical Journal, 2019. doi:10.1016/j.bpj.2019.06.016.

[25] Anna Pietuch. Membrane mechanics governs cell mechanics in epithelial cell: how surface area regulation ensures tension homeostasis.

[26] Ramesh Prasad and Huan Xiang Zhou. Membrane Association and Functional Mechanism of Synaptotagmin-1 in Triggering Vesicle Fusion. Biophysical Journal, 2020. 119(6): 1255-1265. doi:10.1016/j.bpj.2020.08.008.

[27] Thomas Schlake, Andreas Thess, Mariola Fotin-Mleczek, and Karl Josef Kallen. Developing mRNA-vaccine technologies. RNA Biology, 2012. 9(11): 1319-1330. doi:10.4161/rna.22269.

[28] Hermann B.roder Schmidt and Dirk Görlich. Nup98 FG domains from diverse species spontaneously phase-separate into particles with nuclear pore-like permselectivity. eLife, 2015. 4: 1-30. doi:10.7554/eLife.04251. 
[29] Alpay B. Seven, Kyle D. Brewer, Liang Shi, Qiu Xing Jiang, and Josep Rizo. Prevalent mechanism of membrane bridging by synaptotagmin-1. Proceedings of the National Academy of Sciences of the United States of America, 2013. 110(34): 3243-3252. doi:10.1073/pnas.1310327110.

[30] Serge Mostowy Shenoy and Avinash R. The cytoskeleton in cell-autonomous immunity: structural determinants of host defence structural determinants of host defence. Nature Reviews Immunology, 2016. 15(9): 559-573. doi:10.1038/nri3877.The.

[31] S. J. Singer and Garth L. Nicolson. The Fluid Mosaic Model of the Structure of Cell Membranes. Science, 1972. 175(4023): 720-731. doi:10.1126/science.175.4023.720.

[32] George J. Stanley, Ariberto Fassati, and Bart W. Hoogenboom. Atomic force microscopy reveals structural variability amongst nuclear pore complexes. Life Science Alliance, 2018. 1(4): 1-13. doi:10.26508/1sa.201800142.

[33] Sebastian J. Streichan, Christian R. Hoerner, Tatjana Schneidt, Daniela Holzer, and Lars Hufnagel. Spatial constraints control cell proliferation in tissues. Proceedings of the National Academy of Sciences, 2014. 111(15): 5586-5591. doi:10.1073/pnas. 1323016111.

[34] Thomas C. Südhof. A molecular machine for neurotransmitter release: Synaptotagmin and beyond. Nature Medicine, 2013. 19(10): 1227-1231. doi:10.1038/nm.3338.

[35] R. Bryan Sutton, Bazbek A. Davletov, Albert M. Berghuis, Thomas C. Sudhof, and Stephen R. Sprang. Structure of the first C2 domain of synaptotagmin I: A novel Ca2+/phospholipid-binding fold. Cell, 1995. 80(6): 929-938. doi:10.1016/ 0092-8674(95)90296-1.

[36] Thomas C. Südhof and Josep Rizo. Synaptic Vesicle Exocytosis. Cold Spring Harbor Perspectives in Biology, 2011. 3(12): a005637. doi:10.1101/cshperspect.a005637.

[37] Thomas C. Südhof. The presynaptic active zone. Neuron, 2012. 75(1): 11-25. doi:https://doi.org/10.1016/j.neuron.2012.06.012.

[38] Charles Tanford. The Hydrophobic Effect and the Organization of Living Matter. Science, 1978. 200(4345): 1012-1018.

[39] Jaclyn Tetenbaum-Novatt, Loren E. Hough, Roxana Mironska, Anna Sophia McKenney, and Michael P. Rout. Nucleocytoplasmic transport: A role for nonspecific competition in karyopherin-nucleoporin interactions. Molecular and Cellular Proteomics, 2012. 11(5): 31-46. doi:10.1074/mcp.M111.013656. 
[40] Thomas F. J. Martin. PI(4,5)P2 -binding effector proteins for vesicle exocytosis. Physiology \& behavior, 2018. 176(1): 139-148. doi:10.1117/12.2549369.Hyperspectral.

[41] Daniel Ungar and Frederick M Hughson. SNARE PROTEIN STRUCTURE AND FUNCTION. Annual Review of Cell and Developmental Biology, 2003. 19: 493-517. doi:10.1146/annurev.cellbio.19.110701.155609.

[42] Susan R. Wente and Michael P. Rout. The nuclear pore complex and nuclear transport. Cold Spring Harbor perspectives in biology, 2010. 2(10): 1-19. doi:10.1101/ cshperspect.a000562.

[43] Zhen Zhang, Enfu Hui, Edwin R. Chapman, and Meyer B. Jackson. Phosphatidylserine regulation of ca2+-triggered exocytosis and fusion pores in pc12 cells. Molecular Biology of the Cell, 2009. 20(24): 5086-5095. doi:10.1091/mbc.e09-08-0691. PMID: 19828732. 


\section{CHAPTER 2}

\section{INTRODUCTION OF COLLOIDAL PARTICLES AS FORCE AND DISPLACEMENT PROBING SENSOR}

The contrast between macroscopic and microscopic objects is apparent in laboratory observations. For example, a glass marble will sink rapidly in water; however, if we grind it into sub-micron-sized particles, these will float or disperse freely in water, producing a visibly cloudy solution that can remain stable for hours or days. In this process, we have created a colloidal dispersion or solution, which can interact with complex surfaces [28].

Three fundamental forces that operate on small particles in solution can be observed:

- a gravitational force, tending to settle or raise particles depending on their density relative to the solvent

- a viscous drag force, which arises as a resistance to motion, since the fluid has to be forced apart as the particle moves through it

- the kinetic energy of the molecules and particles induces irregular Brownian movement

Each particle, independent of size, will have kinetic energy, on average, of around $1 k_{b} T$. A random kinetic motion will dominate small particles' behavior, which will not settle, and the dispersion will be completely stable. Single particles from these dispersions can be used as colloidal probes when combined with Atomic Force Microscopy [58, 71, 97], Optical Tweezers [43, 82], or Video Particle Tracking [102] to access the visualization and manipulate biological processes otherwise invisible rather challenging to access with high precision [37]. Probes in the micrometer range allow addressing length scales ranging from molecular to mesoscopic sized objects. This chapter introduces three methodical approaches illustrated in Figure 2.1 to realize Colloidal Probe Force 
Spectroscopy and shows how to study different biological systems.

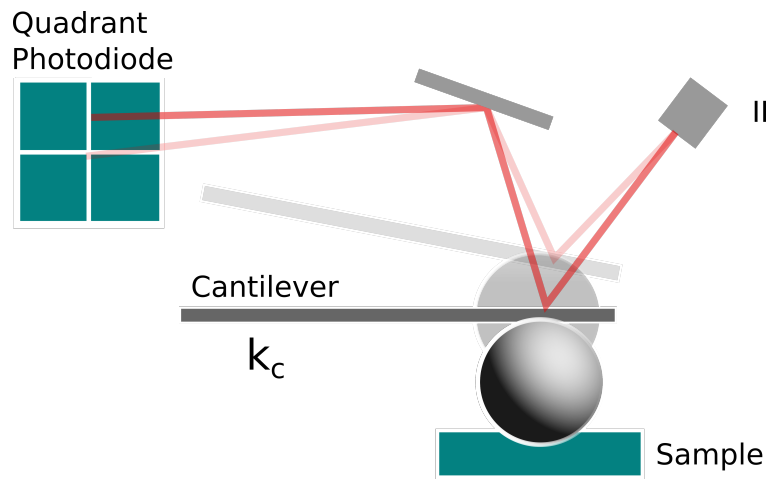

(a) AFM

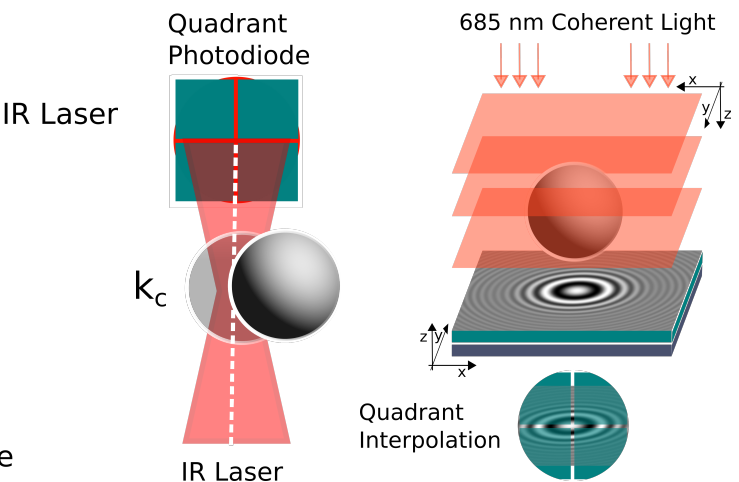

(b) OT

(c) HVPT

Figure 2.1: Schematic illustration of experimental setups to realize colloidal probe force spectroscopy. (a) Atomic Force Microscopy (AFM): an infrared laser beam is aligned on the backside of a cantilever and its reflection is directed via a mirror to a four-quadrant photodiode. (b) Optical Trapping (OT): a colloidal particle is trapped in an optical potential of defined stiffness. A quadrant photodiode records the motion of the particle and its displacement from the trap center. (c) Holographic Video Particle Tracking (HVPT): a microsphere is illuminated by a fiber-coupled collimated red LED (Thorlabs, M455F1, $685 \mathrm{~nm}$ ). Images of holographic diffraction patterns are obtained with a complementary metal-oxide-semiconductor camera. A previously assigned template selects a single diffraction pattern of microspheres for a region of interest of 65 pixels and algorithms, based on cross-correlation and quadrant interpolation are used for determining the position of the bead.

Most strategies to realize Colloidal Probe or Force Spectroscopy experiments, in general, rely on the same principle. A probe is attached to a spring with a known spring constant $k_{c}$ allowing us to calculate the force $F$ by measuring the displacement $\Delta z$ of a spring using Hooke's law $F=k_{c} \Delta z$. The contrast between different force spectroscopic methods is how it realizes the spring and how $\Delta z$ is determined [70].

\subsection{Atomic Force Spectroscopy}

The Atomic Force Microscope (AFM, Fig. 2.2) is a scanning probe microscope. It mainly consists of four parts [12]:

- an AFM controller.

- a piezo-driven 3D-scanner.

- a probe mounted on a flexible AFM cantilever.

- a cantilever-detection detection system based on a laser beam reflected by the 


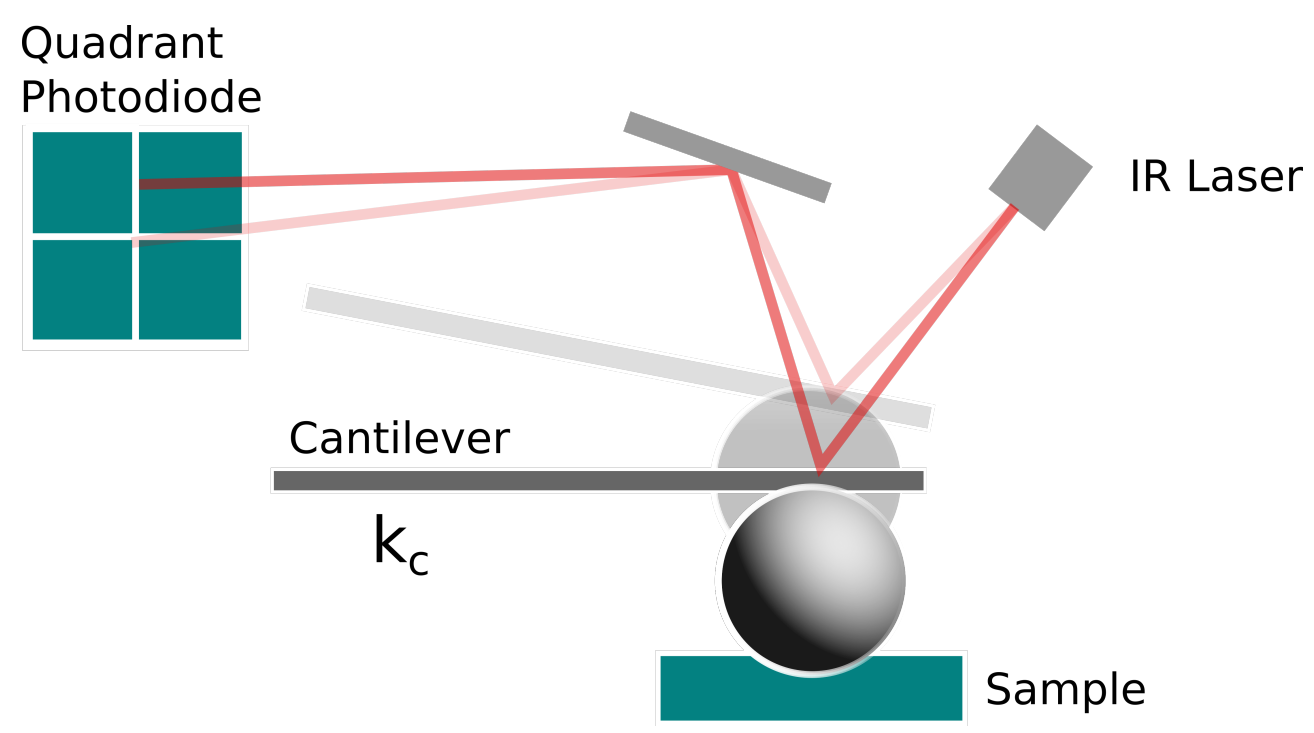

Figure 2.2: Schematic illustration of experimental setup to realize Colloidal Probe Force Spectroscopy by Atomic Force Microscopy: A z-piezo element varies the distance between the spherical tip and the sample surface.

cantilever tip.

In AFM nanoindentation measurements, the AFM cantilever is approached to the sample using the 3D-Piezo-scanner. The contact between samples like cells, a hydrogel, or an solid supported membrane (SSM) and the probe leads to interaction forces detected by a flexible cantilever [19, 23, 54]. Its deflection is sensed via an infrared laser reflected from the cantilever tip to a Position-Sensitive Detector (PSD). Essentially this is a four-segmented photodiode. Each of the four diode segments independently measures the intensity of the incident laser light. A deflection of the cantilever causes a shift of the incident laser spot on the PSD, resulting in changes of the four measured laser intensities. In contrast, the sum of the measured intensities remains constant. Knowing the AFM system's geometry and the Inverse Optical Lever Sensitivity (InvOLS, [m/V]) of the AFM cantilever, the cantilever deflection $d_{c}$ can be calculated differences in laser intensity measured between the diode segments [32, 89].

Typically the InvOLS for a cantilever deflection needed for a difference of one volt is determined by the measurement of Force-Distance Curves on rigid substrates like freshly cleaved mica or glass. By pressing the cantilever against a rigid substrate, the cantilever's deflection $d_{c}$ is equal to the change in the position $z$ of the 3D-scanner, as no indentation appears. Furthermore, as for small deflections, an AFM cantilever follows Hooke's law. The resulting deflection $d_{c}$ is linearly proportional to the interaction force $F_{c}$ acting on the cantilever. 


$$
F=k_{c} \cdot d_{c}
$$

As described above, a cantilever's specific spring constant is needed to determine the force and the strength of a bond between two or more molecules. Generally, cantilever with spring constants between $0.001 \mathrm{~N} / \mathrm{m}$ and $40 \mathrm{~N} / \mathrm{m}$ are commercially available. However, since the spring constant depends on the manufactured shape and size of the cantilever, it has to be calibrated before each measurement to calculate the force [14]: For this purpose, the thermal noise method is used. First, the cantilever is pressed on a clean, rigid substrate, and a force-distance curve is recorded. From the slope of the curve's contact region, the sensitivity in units of $\mathrm{nm} / \mathrm{V}$ can be obtained to convert the photodiode signal into the cantilever bending. By recording the thermal noise of the cantilever, the specific spring constant can be determined [32].
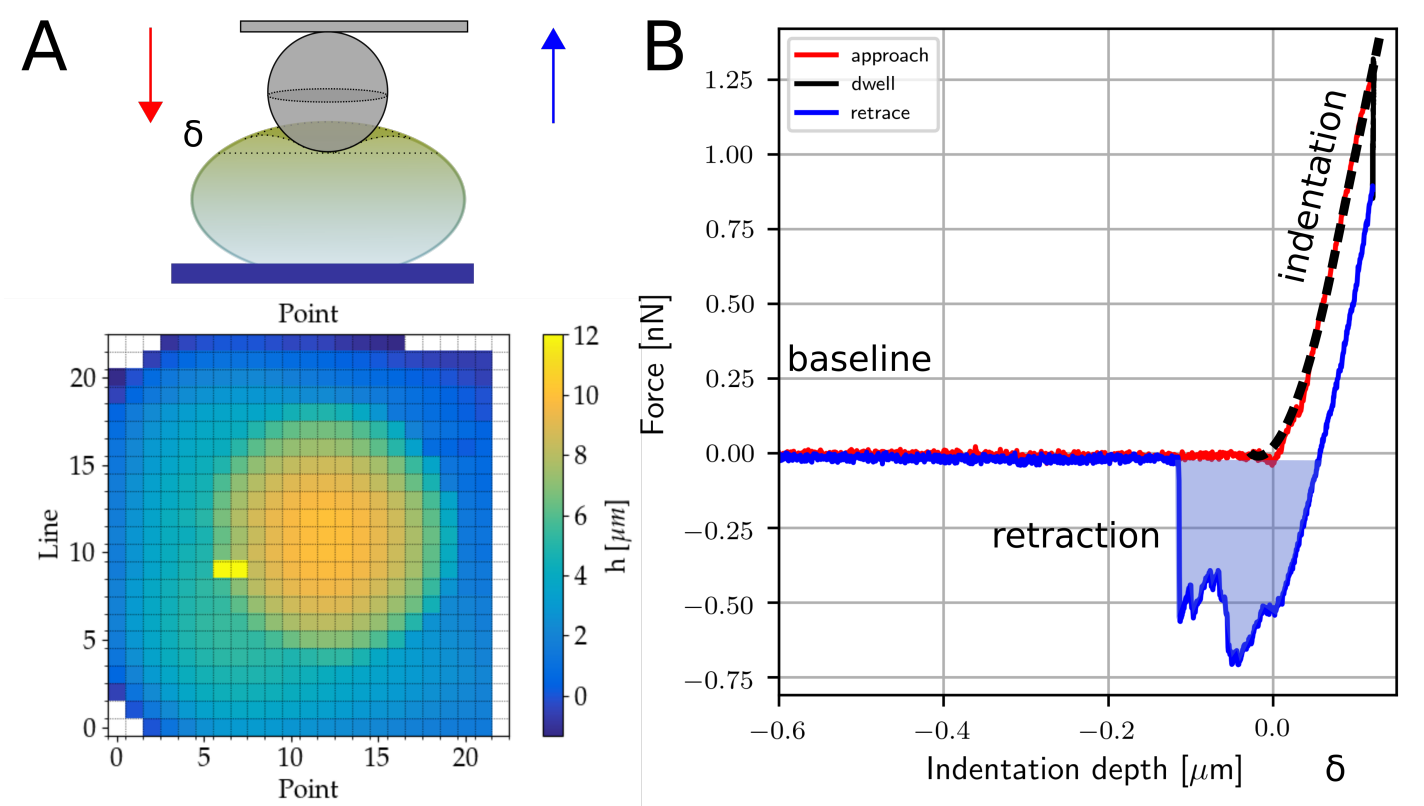

Figure 2.3: Experimental approach for the mechanical evaluation of 2-dimensional spatial indentation experiments. A: Force-Map of Young-Moduli at different points of the underlying sample. Force-map height-profile derived at a set-point of $1 \mathrm{nN}$ from indentation (bottom). B: Evaluation by different mechanical models applied to a selected force indentation curve. Mechanical models are hertzian contact, bilodeau, sneddon-, tension-model and a neo-hookean approach.

The force-distance curve in Figure 2.3 shows that after the first contact with a soft biological sample, the force on the tip increases. After reaching the force setpoint, the cantilever is dwelled or immediately retracting again from the surface. In this retraction, many different adhesion events can be observed. We can gain information 
about how deep the cantilever tip penetrates the sample from the trace curve. The so-called indentation depth $\delta$ is defined as the subtraction of the measured cantilever deflection $d$ from the z-piezo extension $z$ :

$$
\delta=\left(z-z_{0}\right)-\left(d-d_{0}\right)
$$

$z_{0}$ is the position of the z-piezo, where the tip first comes into contact with the sample surface. With this indentation depth, it is possible to calculate the sample's elastic properties from the measured force indentation curve. To fit the curves, different contact mechanical models are used [12, 101], which provide the elastic Young's modulus E. The two most commonly used mechanical models are the Hertzian model and the Sneddon model [46]. The Hertzian model is valid for a cantilever with a spherical indenter on a flat surface and is described as:

$$
F_{\text {Hertz }}=\frac{4}{3} \frac{E}{\left(1-v^{2}\right)} \sqrt{R_{\text {tip }}} \delta^{\frac{3}{2}}
$$

With the radius of the tip $R_{\text {tip }}$ and $v$ for the Poisson's ratio of the sample. For a conical indenter, the Sneddon model is used [36, 87] with the opening half-angle $\alpha$ of the cone:

$$
F_{\text {Sneddon }}=\frac{2}{\pi} \frac{E}{\left(1-v^{2}\right)} \tan (\alpha) \delta^{2}
$$

Sneddon's model follows the classical Hertzian theory for the indentation of an infinite, purely elastic half-space with a uniformly defined conical indenter. For pyramidal indenters, a corrected solution was introduced by Bilodeau [7]. With a tip-angle of $\theta$ following equation obtains the elastic modulus:

$$
F_{\text {Bilodeau }}=\frac{3}{4} \frac{E}{\left(1-v^{2}\right)} \tan \theta \delta^{2}
$$




\subsection{Mechanical evaluation of indentation experiments}

The systematic exploration of biological tissue through indentation can be described mechanically by viscosity, plasticity, and elasticity [27, 35, 57]. Theoretical considerations and derivation in section 2.2.1, 2.2.3, and 2.2.4 are adopted from Moeendarbary et al. [65].

Viscosity describes the time-dependent resistance of liquids and gases to deformation when force is applied, known as viscosity loss. A liquid that shows purely viscous behavior is called a Newtonian fluid. Their relationship between $\sigma$ and the timedependent strain or Shear rate $\gamma$ is positive and linear. The associated slope is viscosity.

Elasticity describes a body's property to be reversibly deformed, i.e., return to the original structure after the end of a force. The slope of an associated stress-strain curve is constant and positive as described to Hook's law that the spring force $F$ depends on the spring constant $\mathrm{k}$ and the deformation $x$ writes, the modulus of elasticity or Young's modulus $E$ describes the relationship between $\sigma$ and $e$.

The elastic moduli of soft media were previously determined by many researchers using rubber elasticity and viscoelasticity theories. The medium was assumed to be a linear, elastic, isotropic, incompressible material, and Poisson's ratio was considered equal to one-half. Most methods employed are compression, macroscopic indentation, atomic force microscopy, or manipulation of embedded spherical beads.

All measurements were performed using a NanoWizard II, IV TM AFM (JPK Instruments) or a MFP-3D ${ }^{\mathrm{TM}}$ (Asylum Research) setup mounted on top of an Olympus IX81 microscope. For colloidal probe force spectroscopy and indentation experiments, forceindentation-curves were recorded at room temperature using cantilevers with a sphere attached to the tip. Either a borosilicate glass microsphere (Duke Scientific Corporation, Palo Alto, Canada) with a diameter of $15 \mu \mathrm{m}$ was glued to a tip-less cantilever or pre-manufactured (CP-PNP-SiO sCube, Germany) cantilevers were used.

\subsubsection{Hertzian contact mechanics: indentation of elastic material [65]}

Here the Landau and Lifshitz approach is presented for studying the contact problem for spherical linear isotropic elastic bodies, which is known as Hertz's contact problem [52]. It can be shown that when two smooth surfaces contact each other without deformation, one can express separation $h$ along the z-direction in terms of their principal relative radii of curvature. For the contact of two spheres with radii of $R_{1}$ and $R_{2}$, the separation takes the form: 


$$
h \approx \frac{1}{2}\left(\frac{1}{R_{1}}+\frac{1}{R_{2}}\right)\left(x^{2}+y^{2}\right)=\frac{\left(x^{2}+y^{2}\right)}{2 R^{*}}
$$

where $\mathrm{x}$ and $\mathrm{y}$ are coordinates lying on the tangent plane of the contact with the contact point as the origin and $R^{*}=R_{1} \cdot R_{2} / R_{1}+R_{2}$ the relative radius of curvature.

Now let us apply a total compressive load of $F$ to the touching spheres such that the centers of the two spheres approach one another by an amount $\delta=\delta_{1}+\delta_{2}$. The separation distance between two bodies at a given height of $z$, can be calculated.

Considering the Hertzian conditions, i.e., continuous, non-conforming, and frictionless surfaces, small elastic strains where the radius of contact circle $a$ is much smaller than the relative radius of curvature $a<<R^{*}$ and that one can view each sphere as an elastic half-space. One can write the normal displacement of each surface in terms of pressure $P$ on the surface.

All parameters can be expressed in terms of the total load $F$ as in practice this parameter is specified:

$$
F=\frac{8}{3} \frac{\mu^{*}}{R^{*}} a^{3}=\frac{8}{3} \mu^{*} \sqrt{R^{*}} \delta^{3 / 2}
$$

where $a=\sqrt{R^{*} \delta}$ the contact radius and $\mu^{*}$ as the equivalent shear modulus.

$$
\frac{1}{\mu^{*}}=\frac{1-v_{1}}{\mu_{1}}+\frac{1-v_{2}}{\mu_{2}}
$$

One particular case of the Hertz contact problem, which is of interest to us, is where one sphere is rigid $\mu_{1} \rightarrow \infty$ and of finite radius $R_{1}$ and the other sphere is elastic $R_{2} \rightarrow \infty$. This is the case of indentation of a semi-infinite object, and for this case, the force-indentation relationship is:

$$
F=\frac{8 \mu_{2}}{3\left(1-v_{2}\right)} \sqrt{R_{1}} \delta^{3 / 2}
$$

For measurement of indentation depth, the approach of AFM force-distance curves was analyzed to extract the elastic modulus $E$ which is linearly related to the shear modulus 
$G$ through the Poisson ratio $E=2 G(1+v)$. For estimation of the elastic modulus, we assumed a Poisson ratio $v=0.3-0.5$. The contact point between the tissue and the AFM tip was found using the method described before. The indentation depth $\delta$ was calculated by subtracting the cantilever deflection $d$ from the piezo translation $z$ after $\operatorname{contact} \delta=z-d$. E was estimated by fitting the curve's contact region with a Hertzian contact model between a sphere and an infinite half-space. The relationship between the applied force $F$ and the indentation depth is:

$$
F=\frac{8}{3} \frac{G}{1-v} R^{1 / 2} \delta^{3 / 2}
$$

with $v$ the Poisson ratio, and $R$ the radius of the spherical indenter. To minimize errors arising from finite cell thickness, we excluded data points where the indentation depth $\delta$ was more than a quarter of the sample height $h$ [17].

\subsubsection{Tension measurements through membrane tether formation}

In this work, planar solid-supported and cellular membranes were used to mimic the membrane topology of fusion in the biological systems at different membrane tension. For this purpose, the fluid membrane is viewed as a two-dimensional elastic body that expands until the interaction of the membrane with its environment is in equilibrium with the repulsion of individual lipids with one another [5, 100]. This minimization of the surface energy determines the possible ratio of membrane area to lipid molecule, so that a DOPC molecule at $30^{\circ} \mathrm{C}$ has an area of $72.4 \pm 0.5 \AA$, for example [50]. Elastic bodies generally have the Property of reversibly changing shape under the action of an external force. If the deformation is small, Hook's law applies.

This force can deform the membrane in several ways. Possible examples of membrane deformation are:

- bending

- shear

- thickness compression

- lateral extension and compression

Using a planar model membrane, the following should only refer to the elongation. The elastic energy that has to be expended to stretch an unstretched membrane with an 
area $A_{0}$ to $A>A_{0}$ is given by

$$
E_{\text {stretch }}=\frac{1}{2} K_{A} \frac{\left(A-A_{0}\right)^{2}}{A_{0}}
$$

The area compressibility module $K_{A}$ is the proportionality constant between the area's quadratic deflection from its unstretched state and its corresponding energy. The compressibility modulus describes the force needed to increase the intermolecular distance between the lipid molecules. With the surface tension of the interface between water and the aliphatic $\gamma=50 \mathrm{mN} \mathrm{m}-1$, the compressibility module for a lipid monolayer is $K_{A}=2 \gamma$, and in a first approximation for a lipid bilayer $K_{A}=4 \gamma$ [3]. For phospholipids, $K_{A}$ 's typical values are measured in the range between $230-250 \mathrm{mN} \mathrm{m}-1$. With a constant number of lipids $N$, the membrane tension $\tau$ is given by the derivation of the energy according to the area [39, 56, 78]:

$$
\tau=\left(\frac{\partial E_{\text {streteh }}}{\partial A}\right)_{N}=K_{A} \frac{A-A_{0}}{A_{0}}
$$

There is a linear relationship between the tension and elongation of the membrane with the dimensionless relative change in area $\epsilon=\left(A-A_{0}\right) / A_{0}$, like the relation in Hook's law.

$$
\tau=K_{A} \cdot \epsilon
$$

The membrane tension and the area compressibility module can be determined experimentally in different ways. Several methods have become established to measure membrane tension[2]:

- Compression of cells or vesicles between two plane-parallel discs

- Aspiration using micropipettes

- Adding lipid material and changing the osmolarity

- Pulling on membrane tubes with atomic force microscopes or optical tweezers 
The latter methods, in particular, can be used to change the membrane tension. The membrane tension regulates the balance between endocytosis and exocytosis. If the membrane tension is high, lipid material is introduced into the membrane by fusion during exocytosis, and the membrane tension is thus reduced. In endocytosis, membrane tension is increased by removing lipid material. It could be shown on free-standing membranes that the tension serves as a driving force for expanding fusion pores [22].

Here, force curves were analyzed according to the tension model in the cells' context. In this model, the cell is viewed as a pre-stressed surface around a liquid body. In a micropipette setup, the surface tension of cells originates from the shell built up by the cytoskeletal cortex and the surrounding membrane [26, 77, 85].

For the origin of the isotropic tension $T$, the cells pre-tension $T_{0}$ and the area dilatation upon indentation has to be taken into account:

$$
T=T_{0}+K_{A} \frac{\Delta A}{A_{0}}
$$

$K_{A}$ is the area compressibility modulus, and $\Delta A$ is the area change compared to the initial area $A_{0}$. For large area expansion, membrane from reservoirs such as protrusions or caveolae has to be recruited as the membrane would otherwise rupture under the elongation of more than $\approx 2-3 \%$ [68].

For small indentation $T_{0}$ is predominant which composes the membrane tension $T_{m}$ and tension contributions from the contractile actomyosin cortex $T_{\text {act }}$ [29, 77].

$$
T_{0}=T_{m}+T_{a c t}
$$

Excess membrane material $\mathrm{A}_{\mathrm{ex}}$ is stored in wrinkles and infoldings. This additional material can accommodate deeper indentation of the cell with an AFM tip and hinders lysis of the plasma membrane. To take this effect into account, $\mathrm{K}_{\mathrm{A}}$ is replaced by an apparent area compressibility modulus $\tilde{K}_{A}$; cf. Pietuch and Brückner [76]

$$
\tilde{K}_{\mathrm{A}}=\tilde{K}_{\mathrm{A}} \frac{A_{0}}{A_{0}+A_{\text {ex }}}
$$


Before analyzing the experimental data, the force curves' baselines were corrected for the overall offset and slope of the individual force-distance-curve (FDC) and the contact point determined by visual inspection. After indentation, force curves that did not show a distinct contact point or exhibited an unusual slope in force were excluded from the analysis. The contact regimes of the processed curves were fitted with a polynomial function (see equation 2.17), calculating the Force $(F)$ from the indentation depth $(d)$, using coefficients depending on the apparent cellular pre-tension and the apparent area compressibility modulus $\left(\tilde{K}_{A}\right)$ as fit parameters. $\tilde{T}_{0}$ and $\tilde{K}_{A}$ can be transformed into the pre-tension $T_{0}$ and the area compressibility modulus $K_{A}$ by geometrical factors

$$
F_{(d)}=\tilde{T}_{0} \cdot d+\tilde{K}_{A} \cdot d^{3}
$$

Indentation, though, is not the only way to probe the mechanical properties of biological samples. Pulling out small nanotubes provides further details of the mechanical behavior of cells [77]. The force $F_{t}$ measured during tether rupture is related to the membrane tension as indicated in Equation 2.18. It combines contributions from the membranes' in-plane tension and contributions from the cytoskeleton's attachment to the membrane. Hochmuth described the first experiments of tether pulling out of red blood cells in 1982 [29]. Besides micropipette aspiration, magnetic or optical tweezers, membrane tether can be drawn out of a membrane using an atomic force microscope. For this purpose, a strong binding between tip and membrane is required. Lectins, like Concanavalin A (Con A), are regularly used for tip functionalization. They bind to specific sugars at the plasma membrane and enable attachment and the deformation membrane [98].

After indenting the membrane with a functionalized probe, a strong interaction between tip and sample occurs. A small tether is pulled out during retraction, reflected in a constant force in the curve's force-distance plateau. At a certain point, the tether ruptures, resulting in a specific step in the force-distance curve. One can obtain the tether length and the rupture force from these measurements.

The tether rupture force $\mathrm{F}_{\text {tether, }}$, measured at constant pulling velocity $v$ can be written as shown in Equation 2.18

$$
F_{\text {tether }}=2 \pi \sqrt{2 \kappa \cdot t_{t}}+\frac{2 \pi \eta v_{t}}{C}
$$


where $\eta$ describes the membrane viscosity and $C$ is a correction factor [10]. A typical value used for cell membranes is $C=1.6$. As an approximation, viscous contributions described by the second term can be neglected. The bending rigidity $\kappa$ depends on the amount of cholesterol in the membrane. When $\kappa$ is known, the membrane tension can be calculated. A typical value of $\kappa=2.5 \cdot 10^{-19} \mathrm{~J}$, representing fluid lipid bilayers, is used to calculate the membrane tension [40, 86].

\subsubsection{Linear viscoelasticity $[65$}

A viscoelastic material undergoing deformation simultaneously stores and dissipates mechanical energy. Viscoelasticity is the phenomenological theory which describes the time-dependent response of such material. In this framework, regardless of the material's microstructure, the relaxation processes are represented by exponential or power-law functions of time [1, 27] as observed in force-time curves by AFM indentation experiments, which is shown in Figure 2.4.

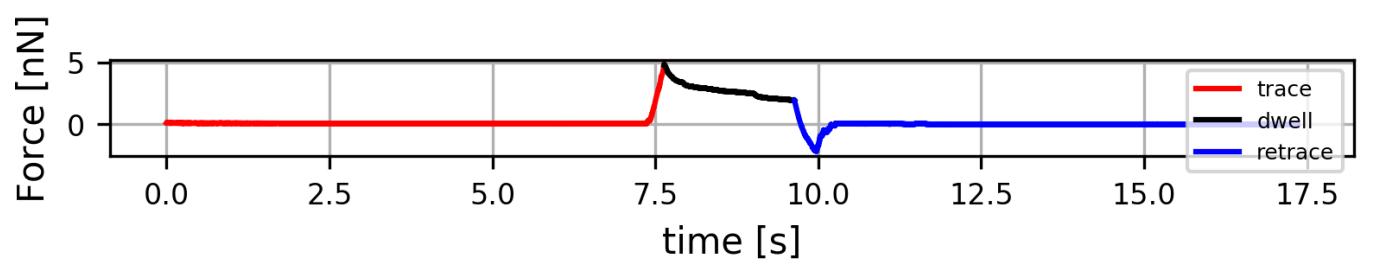

Figure 2.4: Schematic illustration of indentation experiment from AFM as a plot of force vs. time: approach trace (red), dwell contact time of $3 \mathbf{s}$ (black) and retraction retrace (blue) . Time-dependent force ralaxation process during dwelling of contact is observed.

In models based on exponential relaxation processes, stress or strain functions can be represented by combining spring and dashpot elements. In such models, the resultant exponential functions can have a single relaxation time if there is only one dashpot element or relaxation time distributions if there are several [57]. On the other hand, another type of relaxation model that cannot be represented by simple mechanical analogs but is encountered experimentally widely in complex materials dynamics is power-law relaxation. Most of the work to date uses a viscoelastic description that suggests that a single-phase homogenous substance is apparent [27]. Although these models match well with experimental evidence, they do not relate the rheological properties measured to all the tissue structural or biological parameters. An alternative investigation is based on the poroelasticity framework [30, 72, 88]. 


\subsubsection{Poroelastic indentation [65]}

Spherical nanoindentation has become a standard method for the mechanical characterization of materials, including materials with time-dependent behaviour, such as hydrogels [45, 72, 96]. Time-dependent behavior has a considerable influence on the mechanical response of high compliance materials. Most nanoindentation studies incorporated viscoelastic and viscous-elastic-plastic models. Polymers i.e. are known to exhibit viscoelastic behavior, but it has recently been suggested that hydrogels, such as those used in the manufacture of contact lenses, exhibit response more akin to poroelastic materials [1, 20, 83]. Conventional engineering methods such as compression, tensile and shear tests have limitations for the characterization of very soft materials due to difficulties in accessing small samples. Furthermore these methods do not have the necessary accuracy and sensitivity to study the time-dependent dynamics of soft materials with short time scale responses. One of the best methods for characterization of very soft materials, especially gels or cells, is an indentation test conducted with AFM indentation experiments to study rheology in the framework of visco- and poroelasticity [18, 66, 69].

The theory of poroelasticity studies the behavior of a porous medium consisting of an elastic solid matrix infiltrated by an interconnected network of fluid saturated pores [84].

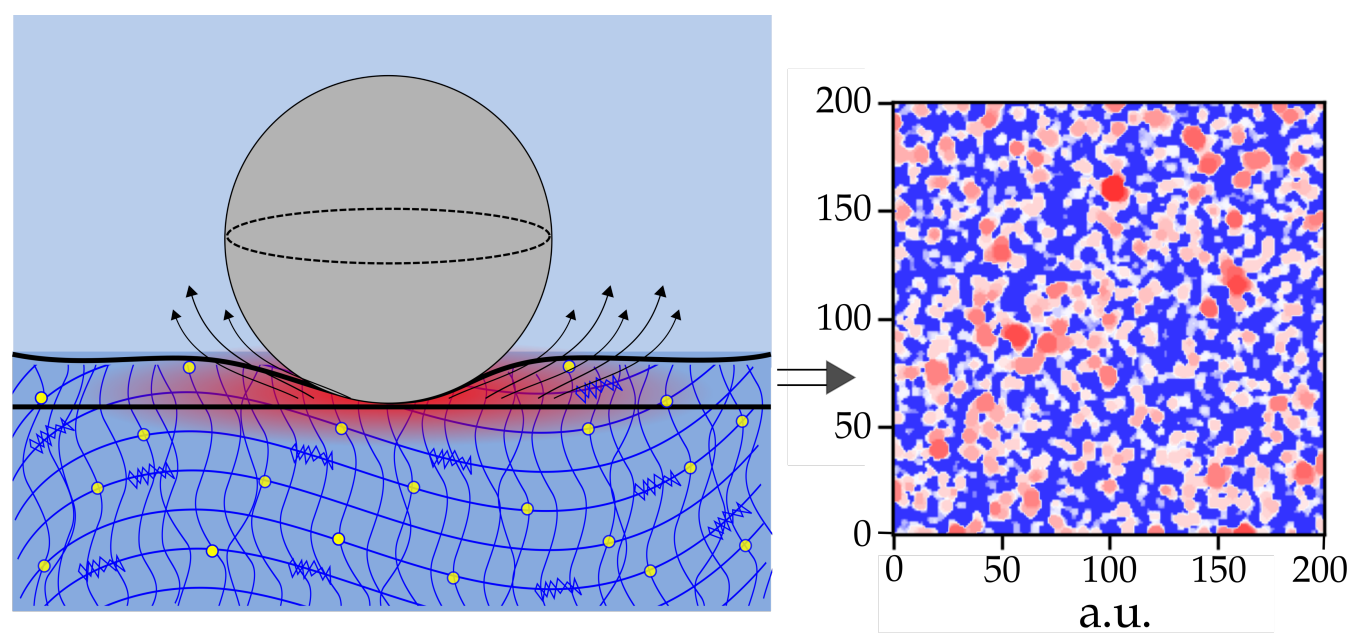

Figure 2.5: Schematic illustration of an indentation experiment of a poroelastic material with a spherical AFM-tip. Increased pore pressure is reduced through the re-distribution of water content inside from the FG-hydrogel after application of a loading force by AFM-indentation (left). Artificial image of porous materials for illustration generated by PoreSpy (right) [24].

A critical consequence of considering a poroelastic tissue is that the measured macroscopic mechanical properties can be related to some coarse-grained microstructural 
parameters. The hydraulic pore size $\xi$ of a tissue can be determined as a first step to understanding a body's microstructure by applying linear isotropic poroelasticity. The following constitutive equations are adopted from Moeendarbary [66] and are an extension of linear elasticity to poroelastic materials first introduced by Biot [61, 84].

As poroelastic parameters assume their limiting conditions, the Biot formulation can be simplified. The material exhibits its greatest poroelastic effect under the incompressible constituents condition, and the Biot poroelastic theory can be mathematically converted into the biphasic model [69]. We consider the quasi-static process with constant porosity of the isotropic, totally saturated poroelastic medium. The constitutive equation relates the total stress tensor $\sigma$ to the solid phase infinitesimal strain tensor $\epsilon$ and the pressure of the pore fluid $p[16,61,66,84]$ :

As a first step, we assume that pores within the solid matrix have an average radius of $\xi$ to understand the relationship between microstructure and hydraulic permeability [72]. A basic comparison between a Poiseuille flow with radius $\xi$ within a tube and flow through the porous media with porosity $\varphi$ leads to the following hydraulic permeability relationship $K$ :

$$
K=\frac{\varphi}{4 \kappa} \frac{\xi^{2}}{\mu}
$$

where $\mu$ is the viscosity of the fluid and $\kappa$ is a constant taking into account the irregularity, interconnectivity and tortuosity of the pores as depicted in Figure 2.5 (bottom). In the framework of poroelasticity the viscoelastic properties of the sample are observed by the time needed for redistribution of fluids in response to applied mechanical stresses. As the response of the sample to force application depends here on a single experimental parameter: the poroelastic diffusion constant:

$$
\begin{aligned}
D_{p} & =\frac{2 G_{s}\left(1-v_{s}\right)}{\left(1-2 v_{s}\right)} K \\
D_{p} & =\left(\frac{\left(1-v_{s}\right)}{\left(1+v_{s}\right)\left(1-2 v_{s}\right)} \frac{\varphi}{4 \kappa}\right) \frac{E \xi^{2}}{\mu}
\end{aligned}
$$

As a first approximation, all of the parameters inside the parenthesis can be assumed to be a constant $\alpha$ and the functional dependence of all parameters with respect to the porosity of the structure is neglected. Therefore, a fundamental scaling law for 
poroelastic cytoplasm takes the form [61]:

$$
D_{p}=\alpha \frac{E \xi^{2}}{\mu}
$$

where $\mu$ is interpreted as the interstitial fluid viscosity, and $E=2 G_{s} \cdot\left(1+v_{s}\right)$ the elasticity of the constituent solid network.

For indentation of a purely elastic material, there presently exist several closed-form formulations such as Hertz's equation 2.10 for obtaining cellular elastic properties such as the shear modulus. Some closed-form expressions have also been proposed for characterization of time-dependent viscoelastic materials such as indentation of a standard linear solid known as Zener model of a viscoelastic material. However due to the higher complexity of the poroelastic equations, geometry and boundary conditions, there exists no simple closed-form analytical solution that can be used to fit experimental data.

Therefore in this work, and others [31, 51, 73], an empirical expression (eq:2.26) derived from finite element simulations was employed to fit experimental relaxation responses from AFM indentation experiments to access poroelastic parameters by a spherical indenter and contact radius of $a=\sqrt{R \delta}$ :

$$
\begin{aligned}
F(0) & =\frac{16}{3} G \cdot a \cdot \delta \\
F(\infty) & =\frac{8}{3\left(1-v_{s}\right)} \cdot G \cdot R^{1 / 2} \cdot \delta^{3 / 2} \\
\frac{F_{(t)}-F_{(\infty)}}{F_{(0)}-F_{(\infty)}} & =g\left(\frac{D \cdot \tau}{a^{2}}\right) \\
g(\tau) & =0.491 \exp (-0.908 \cdot \sqrt{\tau})+0.509 \exp (-1.679 \cdot \tau)
\end{aligned}
$$

The comparison of the long time-scale and short time-scale limits enables the Poisson ratio of the solid matrix to be estimated:

$$
F(0) / F(\infty)=2\left(1-v_{s}\right)
$$


Charras et al. has performed the establishment of the experimental conditions for measuring cellular poroelastic diffusion constant on HeLa Cells [66]. The indentation depth increased by an average of $5 \%$ over a $2.5 \mathrm{~s}$ relaxation period after rapid local force application by AFM $6 \mathrm{nN}$ applied during a rise time $t_{r}$ of $35 \mathrm{~ms}$ on HeLa cells, while force relaxed by $35 \%$. Therefore, they supposed that the depth of indentation and the region of contact remained constant. In conclusion, their experimental conditions are those of a problem of force-relaxation.

$$
\frac{F_{(t)}-F_{(\infty)}}{F_{(0)}-F_{(\infty)}}=g\left(\frac{D \cdot \tau}{a^{2}}\right)
$$

In poroelasticity, relaxation is due to the movement of water out of the porous matrix in the displaced area after applying a force. Therefore to measure the poroelastic properties, a regime needs to be formed where deformation is applied faster than water can leave the volume displaced. This can be expressed simply by comparing the rise time $t_{r}$ needed to reach an indentation depth $\delta$ with the time necessary for water to diffuse out from the displaced volume $t_{p} \approx L 2 / D_{p}$ with $L \approx R \delta$ ) with $R$ the indenter radius. In the Charras et al. experiments, an indenter with a radius of $R=7.5 \mu \mathrm{m}$, a standard indentation depth of $\delta=1 \mu \mathrm{m}$, was used, and previous experiments indicated $D_{p} \approx 1$ to $100 \mu \mathrm{m}^{2} \mathrm{~s}^{-1}$ in cells with poroelastic characteristics of $t_{p} \approx 0.1$ to $10 \mathrm{~s}$.

\subsection{Optical Tweezers}

Arthur Ashkin found in 1970 that light can exert forces on micron-sized particles, and thereby particles can be trapped in a light beam [4]. He applied this principle in the upcoming years and invented the first optical trap or optical tweezer. Nowadays the optical tweezers are a widely used measuring instrument in the natural sciences that can manipulate microscopic objects at length scales ranging from $\mathrm{nm}$ to $\mu \mathrm{m}$. A highly focused laser beam exerts a force in the range of piconewtons on dielectric, transparent particles as illustrated in Figure 2.6.

An optical trap allows fixing the position of a small particle without mechanically touching it. It helps to position and arrange small particles very precisely. At the same time, the forces which are acting on the trapped particle can be controlled precisely with sub-nanometer resolution. With force values mostly in the pico-Newton range, therefore much smaller than in typical atomic force microscopy experiments. For example, this allows measuring the RNA polymerase's motor protein steps or forces during 


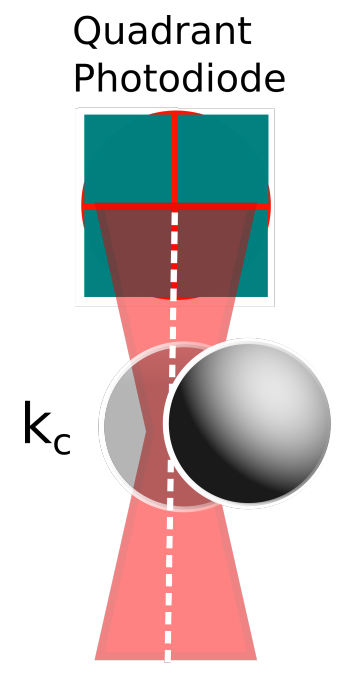

IR Laser

Figure 2.6: Schematic illustration of experimental setups to realize colloidal probe force spectroscopy. Optical Trapping (OT): A colloidal particle is trapped in an optical potential of defined stiffness. A quadrant photodiode records the motion of the particle and its displacement from the trap center.

transcription [79]. The trap produces a harmonic potential. In which the reinstalling force $F$ is linear depending on the particle's displacement $x$ of the particle out of the focal axis. So this behavior follows the same laws again as a typical mechanical spring:

$$
F_{(x)}=k_{x} \cdot x
$$

The proportionality factor is the spring constant of the trap or traps stiffness $k_{x}$ in axial direction. This linear approximation only holds for displacements up to $\approx 200 \mathrm{~nm}$. At large displacements, the sphere will overcome the escape force and leave the trap.

Recording fluctuations and transforming them into Fourier space allows one to analyze the particles movements' power spectral density [11, 91]. It reveals how active the bead is on which time scales as the frequency is reciprocal to time. According to theoretical calculations of the force distributions, one fits the measured power spectral density of a trapped particle with a Lorentz function:

$$
S_{(f)}=\frac{k_{b} T}{\gamma \pi^{2}\left(f_{c}^{2}+f^{2}\right)}
$$


Since $f_{c}=k /(2 \pi \gamma)$ one obtains $k$ from the corner frequency. $S$ is calculated by comparing the measured power spectrum in units of $\mathrm{V} / \mathrm{Hz}$ with the expected power spectrum in $\mathrm{nm} / \mathrm{Hz}$ from the assumption of a spherical particle with a given size in a medium with defined viscosity.

$\gamma$ is the coefficient of friction for the particle in the liquid and results from the hydrodynamic radius $R_{0}$.

$$
\gamma=6 \cdot \pi \eta \cdot R_{0}
$$

According to the fluctuation-dissipation theorem, these particles' equilibrium thermal 'Brownian' motions are linked to the surrounding medium's properties. A simple way to measure this is to track the movement of these particles and calculate a mean squared displacement (MSD), which gives direct accessible information [90]:

$$
M S D=\left\langle r_{(\tau)}^{2}\right\rangle=\frac{1}{T-\tau} \sum_{t=1}^{T-\tau}\left(r_{(r-\tau)}-r_{(t)}\right)^{2}
$$

As illustrated in Figure 2.6, a quadrant photodiode placed in the back-focal plane of the microscope of a laser trap provides a high-resolution position sensor. In addition to the lateral displacement of a trapped sphere, its axial position can be measured by the ratio of the intensity of scattered laser light to the total amount of the light reaching the detector. This offers true three-dimensional position detection of particles in aqueous solution, creating, together with a position control, a photonic force microscope with nanometer spatial and microsecond temporal resolution. The measured position signals are explained as interference of the unscattered trapping laser beam with the laser light scattered by the trapped bead.

\subsection{Free and confined diffusion of colloidal probes in solution}

This section describes the diffusive motion of free and tethered particles. The diffusive motion of a tethered particle can in the limit of short timescales be described as free diffusive motion. At longer timescales the diffusive motion deviates from free diffusion, as the tether confines the particle to an attachment point. Furthermore, the proximity of the particle to the substrate leads to hydrodynamic interactions and to adhesion 
between the particle and the substrate, for which the diffusion constant needs to be corrected [41]. The expectation value of the diffusive excursion of a free particle in bulk liquid is described by the equation:

$$
\left\langle r^{2}\right\rangle_{\text {normal }}=2 n D t
$$

where is given the position of the particle at a time from its starting point, observed in $n$ dimensions, and its motion is described by the diffusion constant $D$ of the particle in the liquid. The diffusion coefficient originates from the general definition $D=\mu \cdot k_{b} \cdot T$, in which $\mu$ is the mobility of the diffusing element. The mobility is defined as the ratio between the terminal drift velocity and the applied force. For smooth, spherical particles in a low Reynolds number flow, the drag on a particle as function of the particle's speed is given by Stokes' relation:

$$
F_{d}=6 \pi \eta r v
$$

In which $\eta$ is the dynamic viscosity of the fluid, $\mathrm{r}$ is the particle's radius, and $v$ the speed of the particle through the fluid. Using the definition $\mu=\frac{v}{F_{d}}$ of the mobility yields:

$$
\mu=\frac{1}{6 \pi \eta r}
$$

which then yields the Stokes-Einstein relationship:

$$
D=\frac{k_{b} T}{6 \pi \eta r}
$$

for the diffusion of a particle in a fluid. This relationship is valid in a low-Reynolds laminar flow regime for smooth spherical, non-interacting particles. In tethered particle motion measurements, particle tracking is essential, because the particle movements need to be monitored far below the illumination light wavelength with precision in 
nanometers' order [25, 38, 53, 64].

\subsubsection{Holographic video particle tracking}

Standard particle tracking based on quantitative analysis of video microscopy images has become a conventional technique in science and engineering branches. It has numerous applications in biophysics, particularly in magnetic and optical tweezers. Time-resolved particle trajectories obtained from sequences of video images have been used to study the interactions between colloidal particles, address fundamental questions in statistical physics, probe the viscoelastic properties of soft media, and measure the dynamical properties of single polymers [21, 48, 62]. For the observation, three different types of illumination can be used, as shown in Figure 2.7. Brightfield illumination yields dark objects on a bright background and is the most straightforward technique for optical microscopy. In brightfield illumination, the light source is positioned beneath the sample. The light then propagates through the sample and is observed by the objective lens and sensor set above the sample. In fluorescence microscopy, the sample is illuminated with light of a specific wavelength absorbed by the fluorophores, causing them to emit light of longer wavelengths. The illumination light is separated from the much weaker emitted fluorescence through the use of a spectral emission filter enabling specific fluorescent particle tracking of bright spots.

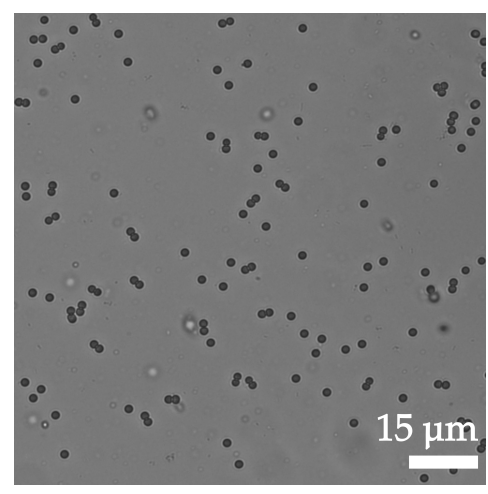

(a) bright field



(b) fluorescent light

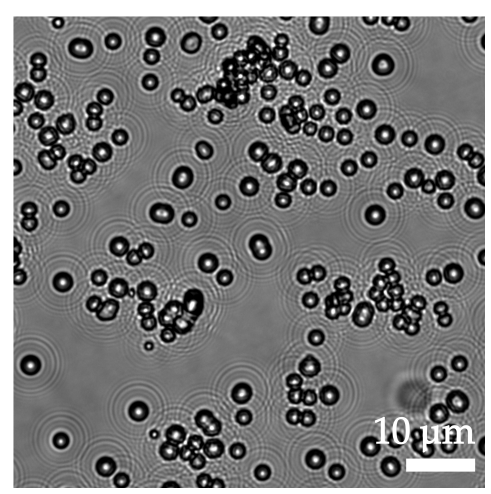

(c) coherent light illumination

Figure 2.7: Comparison of different types of illuminations: bright-field, fluorescent light, and coherent light illumination of micrometer sized silica particles coated with a nanometer thick bilayer containing a fluorescent dye.

The latter type creates holograms, fringe records of light formed by the interference between particles and the light beam by coherent light illumination. They enable the image of thoroughly 3D images. We use Holographic Video Particle Tracking (HVPT) as a tool for three-dimensional particle tracking many objects, namely colloidal probes [53, 93]. 


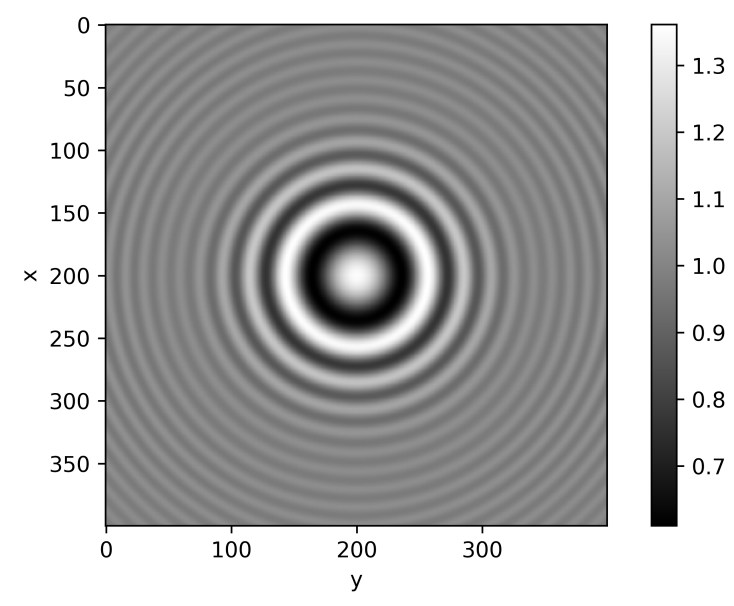

Figure 2.8: Holographic particle pattern. The calculated diffraction pattern of a bead as in-line hologram is generated by a plane wave scattering from a microsphere for medium index of 1.33, illumination wavelength of $685 \mathrm{~nm}$ and a sphere radius of $\mathrm{r}=0.5$ with HoloPy. Arbitrary units and coordinates are used [6]. A collimated laser beam illuminates the sample. Light scattered by the sample interferes with the unscattered portion of the beam in the focal plane of the objective lens. The interference pattern is magnified, recorded and then analyzed to obtain measurements of the particle's position.

Megapixel-size cameras immediately allow the monitoring of multiple objects, and fast acquisition rates enable cameras to be used for single-particle tracking in the $\mathrm{kHz}$ range. A fiber-coupled collimated red LED (Thorlabs, M455F1, $685 \mathrm{~nm}$ ) was used into the imaging path for illumination. A 40x microscope objective (Nikon, CFI Plan Fluor 40x, NA 0.65) was used to image the illuminated microspheres with holographic patterns as shown in Figure 2.8 and 2.7 (c). Images were obtained with a complementary metaloxide-semiconductor (CMOS) camera (UI324xCP-M, 1.280 x 1.024 pixels; Thorlabs) with a full-chip frame rate of $59.8 \mathrm{~Hz}$ [59, 60].

The tracking software used is provided with the stand-alone LUMICKS system [59, 60, 99]. Acquired images were processed in real-time to extract the microsphere positions in three dimensions. A previously assigned template selects the holographic diffraction patterns of microspheres for a Region of Interest (ROI) of 65 pixels, like it is shown in Figure 2.8 and 2.9. Algorithms for determining the bead's position are based on cross-correlation (XCOR) and Quadrant interpolation (QI) ${ }^{1}$ [47, 93]. Before measuring the z-dimension, a lookup table (LUT) was made. Acquired images were processed in real-time to extract the microsphere positions in three dimensions. To determine the $x$ and y position, we applied a cross-correlation algorithm. For the z-position, the LUT was used, which contained a library of radial profiles previously acquired as a function

\footnotetext{
${ }^{1}$ Tracking software is freely available at http://figshare.com/articles/AFS_software/1195874
} 


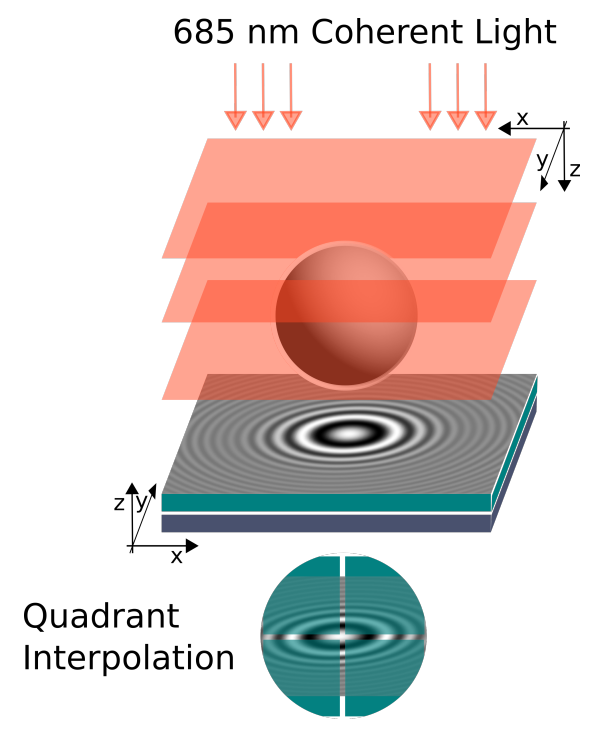

Figure 2.9: Schematic illustration of HVPT setup to realize colloidal probe force spectroscopy. Holographic Video Particle Tracking: A microsphere is illuminated by a fiber-coupled collimated red LED (Thorlabs, M455F1, $685 \mathrm{~nm}$ ). Images of holographic diffraction patterns are obtained with a complementary metal-oxide-semiconductor (CMOS) camera with a full-frame rate of $59.8 \mathrm{~Hz}$. A previously assigned template selects a single diffraction pattern of microspheres for a Region of Interest (ROI) of 65 pixels and Algorithms, based on cross-correlation (XCOR) and Quadrant interpolation (QI) are used for determining the position of the bead.

of microsphere z-position. The precision of $\mathrm{x}$ - and $\mathrm{y}$-position determination was about $1.5 \mathrm{~nm}$, and for z-position determination, it was about $3 \mathrm{~nm}$, at an acquisition rate of $50 \mathrm{~Hz}$ [41, 42].

\subsubsection{Tethered particle motion and analysis of video particle tracking expe- riments}

Tethered particle's motion (TPM) is the motion of a micro- or nanoparticle tethered to a substrate by a macromolecule. It is a system that has proven to be extremely useful for its ability to reveal physical features of the tether because the thermal motion of the bound particle reports sensitively on parameters like the length, the rigidity, or the folding state of its tether [81]. Many experimental methods to probe bonds at the single-molecule level are currently available, including AFM, optical tweezers, magnetic tweezers, and acoustic force spectroscopy. A TPM indicates the probability of the position distribution of the particle (Fig. 2.10) [63]. For a system to become ergotic, sufficient time is needed; then, the position distribution observed accurately reflects the system's position distribution. Parameters that measure the formation of motion patterns will be defined in this section to interpret the shape of motion patterns quantitatively. TPM emerged from single-particle tracking (SPT) [80]. Single-particle- 
tracking is a microscopy technique used to trace, e.g., colloidal particles, organelles in cells, vesicles in and on cells, or (single) molecules labeled with dyes. The tracking of particles is essential in tethered particle motion measurements. The particles can be tracked with accuracy on nanometers' order, well below the illumination light wavelength [63].

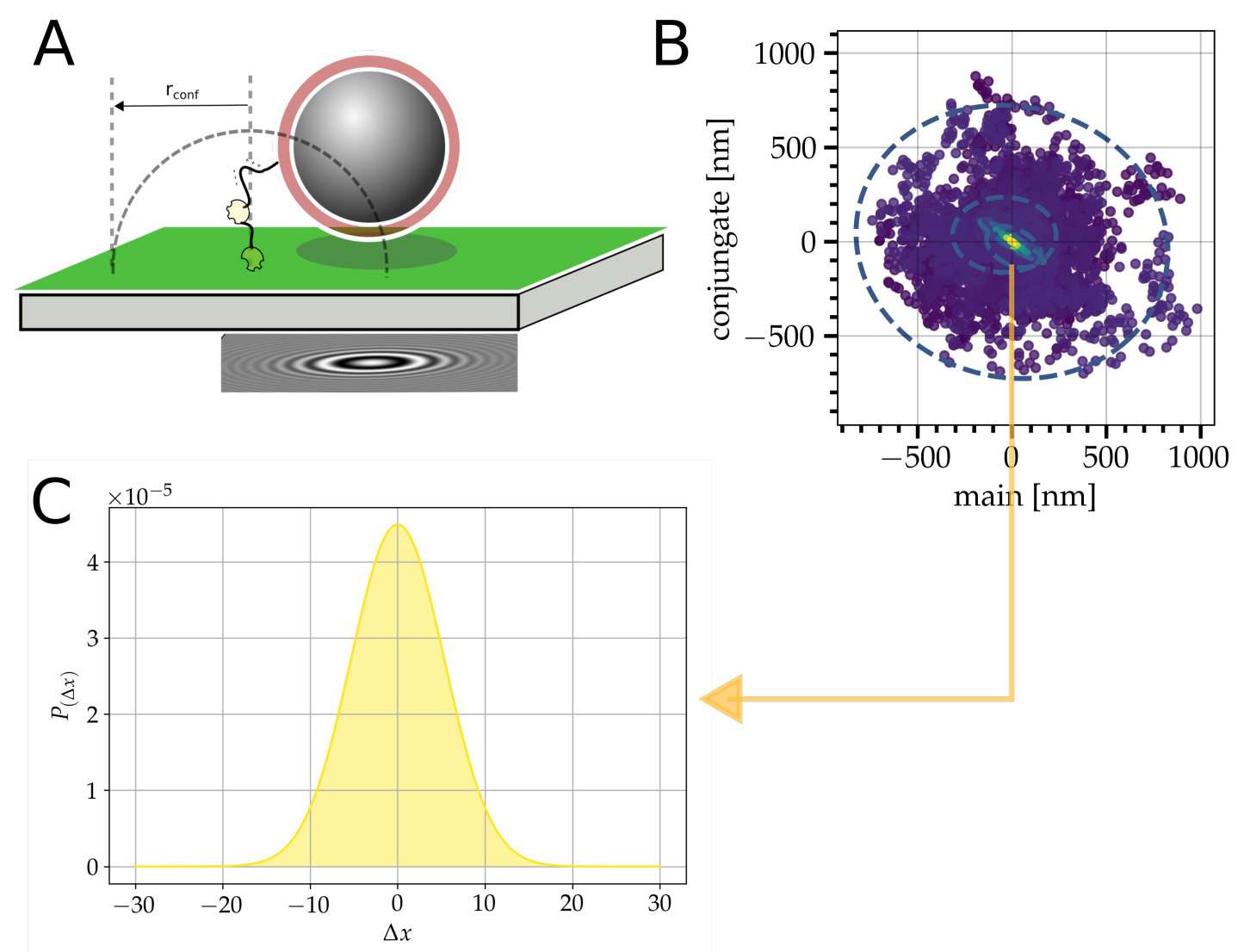

Figure 2.10: Schematic illustration of a tethered particle motion experiment. A: the particle's movement, observed by holographic video particle tracking, is confined through the mechanical length of a single molecule of interest. B: depending on mechanistic detail of the underlying biophysical system different confinent radii and symmetry are observed. C: measured probability of the tethered particle's position distribution resolved from multiple confinements in a time-dependent manner.

The technique is based on an approach for measuring bond kinetics by observing a molecularly tethered particle's motion that can form secondary or tertiary bonds with a substrate [94]. The data obtained in such an experiment in its most unprocessed form consists of a time sequence containing different motion patterns. Blumberg et al. describe a simple method for defining the motion pattern based on the position data's covariance. The covariance matrix is calculated from the position data of the particle [8, 74, 94]. 


$$
C=\left(\begin{array}{ll}
\sigma_{x_{1} x_{1}} & \sigma_{x_{1} x_{2}} \\
\sigma_{x_{2} x_{1}} & \sigma_{x_{2} x_{2}}
\end{array}\right)
$$

Motion symmetry includes the symmetric in-plane motion of a tethered particle around its anchor point and is determined from the covariance matrix in which

$$
\sigma_{x_{i} x_{j}}=\frac{1}{N} \sum_{k=1}^{N} x_{i}^{k} x_{j}^{k}-\bar{x}_{i} \bar{x}_{j}
$$

are the second moments of the bead's position. Here $N$ is the number of video frames and $x_{1}^{k}, x_{2}^{k}$ are the in-plane coordinates (i.e. the position $\mathrm{x}, \mathrm{y}$ ) of the microsphere for frame $k$ as obtained from the drift-corrected data. The eigenvalues $\left(\lambda_{1}, \lambda_{2}\right)$ of the covariance matrix indicate the squares of the major and minor axes corresponding to the in-plane displacement of the bead and are equal for a perfectly symmetric motion.

$$
s=\sqrt{\lambda_{\max } / \lambda_{\min }<1.0}
$$

If first confinement passes this symmetry analysis, TPM motion will serve as a qualified single tether or molecular bond. Multiple tethers may cause asymmetric bead trajectories [94]. Uniformity qualifies tethers based on their motion consistency over time. It eliminates beads showing non-specific binding events, such as binding tether molecules to the bead or glass surface for short periods. To detect these events automatically, one refine a procedure where one first divides the entire time series into a number of subsets labeled by $i=1, \ldots 10$. In subset $i$, a calculate $\mathrm{RMS}_{4 \mathrm{~s}}$ over for example each $4 \mathrm{~s}$ window will be calculated and then averaged, defining $A_{i}=\left\langle R M S_{4 s}\right\rangle_{i}$. Defining a standard deviation of, normalized by the overall average $\mathrm{RMS}_{4} \mathrm{~s}$.

$$
\mathrm{RMS}_{t}=\sqrt{\left\langle(x-\bar{x})^{2}+(y-\bar{y})^{2}\right\rangle_{t}}
$$

Nevertheless, detecting and discriminating events resulting from specific and unspecific binding events, including forming multiple tethers, is crucial for each biological system 
[62, 63, 74, 94]. The dynamic bond formation is crucial when exploring for example mechanistic details on how SNAREs and Syt-1 act together when bilayer merging.
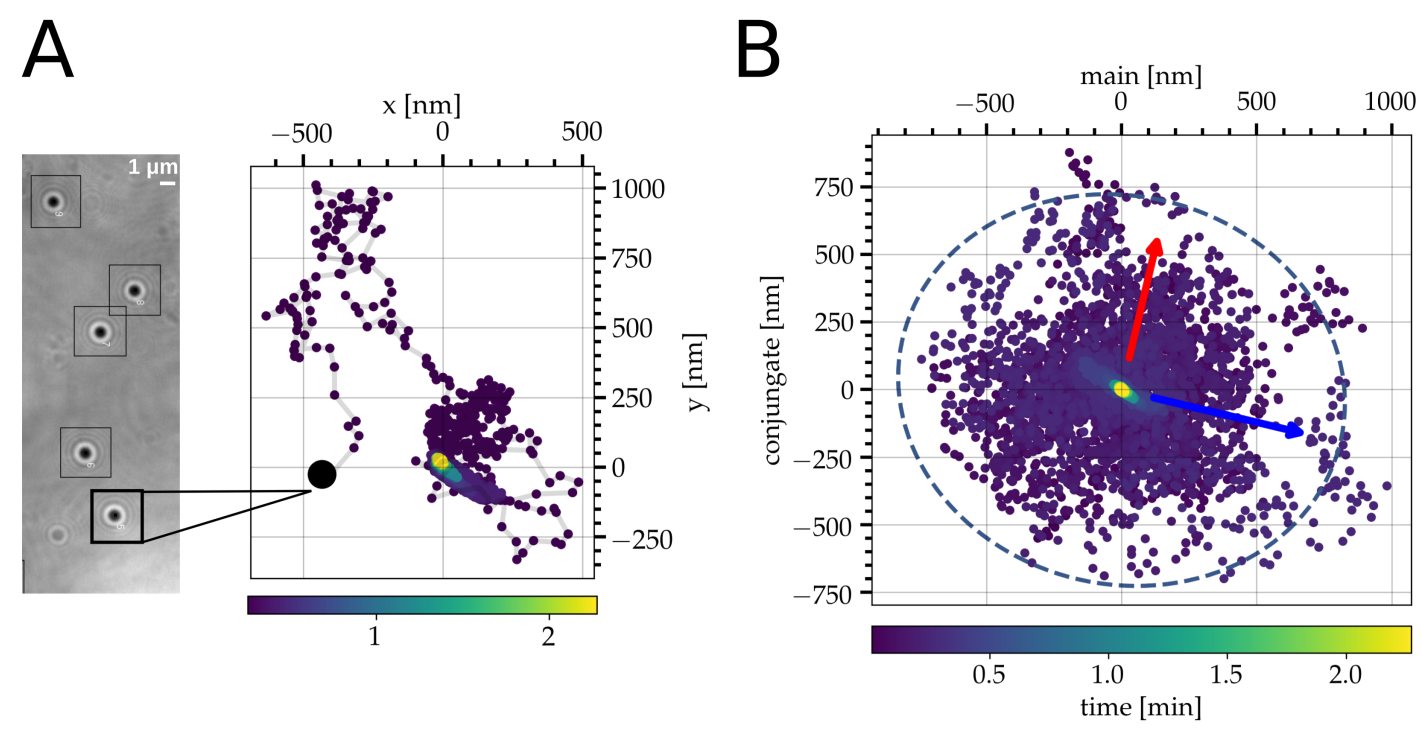

Figure 2.11: Illustration of a tethered particle motion experiment. A: the detection algorithm acting on experimental data. The experimental data provide the motion data as the position of the particle projected onto the xy-plane parallel to the substrate. B: motion corresponding to the position of the particle at one moment in time.

For this purpose, we employed an analysis of the Brownian motion when tethers are formed. The analysis is based on a principal component analysis of de-drifted data and automatic segmentation using a Pruned Exact Linear Time (PELT) method for exact segmentation to detect change points in our dataset (as shown in Figure 2.11).

\section{Brownian Motion of tethered particles}

The diffusive motion of a tethered particle can in the limit of short timescales be described as free diffusive motion. At longer timescales the diffusive motion deviates from free diffusion, as the tether confines the particle to the attachment point. Furthermore, the proximity of the particle to the substrate leads to hydrodynamic interactions between the particle and the substrate [15, 62, 67].

When analyzing TPM experiments and monitoring bond formation, by observing tether particle motion through their MSDs, one must discriminate at least three different cases for diffusive motion, as depicted in Figure 2.12 

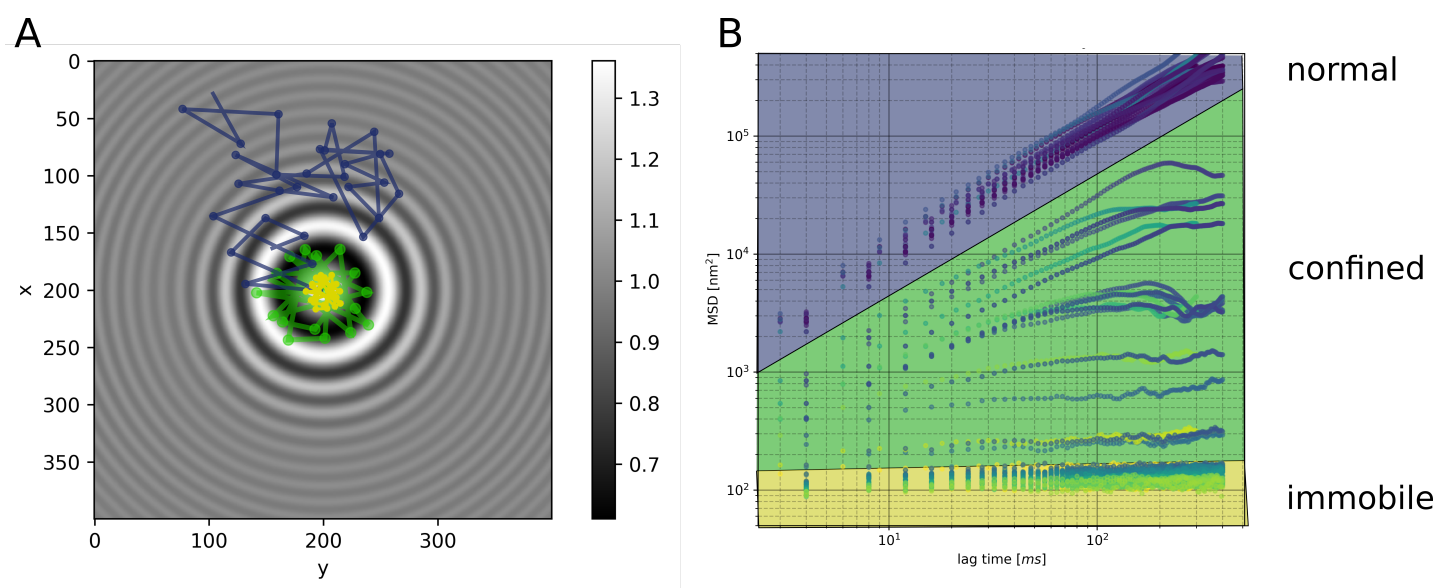

Figure 2.12: Different states of particle motion and their categorization by mean square displacement. A: simulated holographic particle image and schematic trajectories according to normal diffusive (blue), confined (green) and immobile motion (yellow). B: categories of exemplary MSDs obtained from TPM experiments.

$$
\begin{aligned}
\left\langle r^{2}\right\rangle_{\text {normal }} & =2 n D t \\
\left\langle r^{2}\right\rangle_{\text {anomalous }} & =D t^{\alpha} \text { with } \alpha<1 \\
\left\langle r^{2}\right\rangle_{\text {directed }} & =2 n D t+v^{2} t^{2} \\
\left\langle r^{2}\right\rangle_{\text {confined }} & =R_{\text {conf }}^{2} \cdot\left(1-e^{-\frac{t}{\tau}}\right)
\end{aligned}
$$

Confined diffusion occurs when a particle undergoes an interaction that confines the particle to a limited space, such as a particle confined by a tether. The initial diffusion of the particle matches the free diffusion, up to the point in time where the particle starts to experience the effect of the confinement, captured in the parameter $r_{\text {conf }}$. The characteristic confinement time depends on the diffusion coefficient $D$ and the confinement radius. By equating the diffusive speed of a confined particle to a freely diffusing particle at $=0$, the characteristic confinement time is found to be $=R_{\text {conf }}^{2} / 2 n D$ in $n$ dimensions. The diffusion constant can be deducted from the characteristic time $\tau$. Confined diffusion is of special interest for tethered particle motion, because the tether confines the particle to an excursion that relates it to the tether length from the radius of the tethered particle [13, 38, 49, 55, 95].

Adequate observation time is needed to measure complete motion patterns due to the diffusive character of the motion of a tethered particle. A tethered particle's motion is approximated as a particle confined by the tether to a maximum excursion of $R$ from the point of attachment. The diffusive motion is then defined as the diffusive timescale, in which $D$ is the particle's effective diffusion constant near the substrate. 

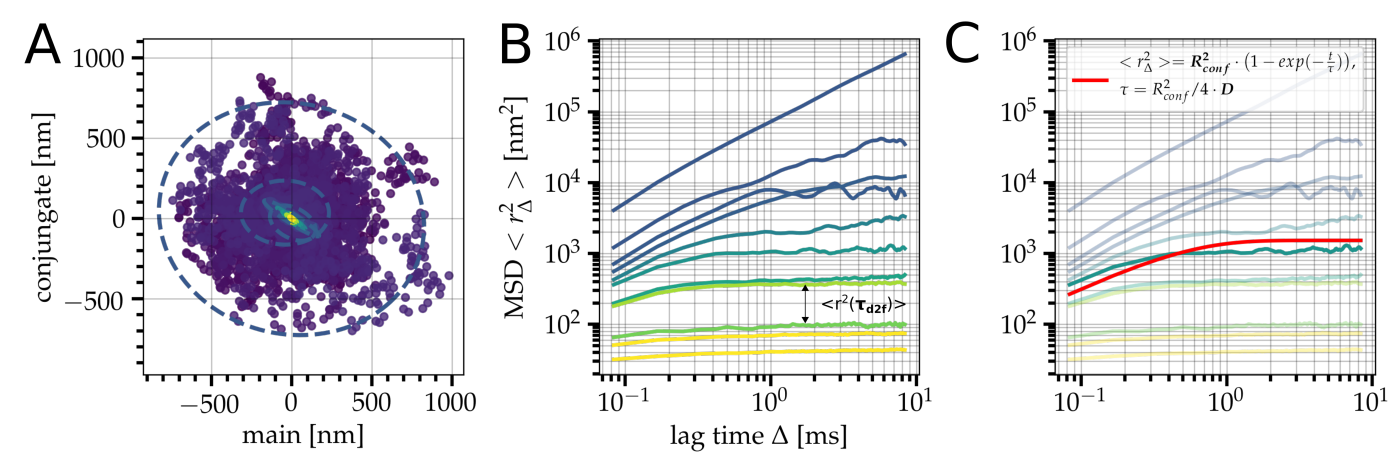

Figure 2.13: Schematic workflow for the analysis of a tethered particle motion experiment. The particle's movement is confined through the mechanical length of a molecule of interest. A: Principal Component Analysis (PCA) and Pruned Exact Linear Time (PELT) reveal segments of different confinements as a function of time used for analysis by their mean square displacements (MSD). B: Color-coded from blue to yellow as a function of time are MSD obtain from each individual segment. C: Fitting of $\left\langle r^{2}\right\rangle_{\text {confined }}=R_{\text {conf }}^{2} \cdot\left(1-e^{-\frac{t}{\tau}}\right)$ reveals the confinement radius $r_{\text {conf }}$ for further characterization.

The diffusive timescale refers to the standard timescale at which the particle's motion begins to sample the pattern of movement uniformly.

Experiments were perfomed in an temperature controlled, integrated flow chamber provided by the LUMICKS AFS TM Stand-alone G2 System or in a 6 channel $\mu$-Slide suitable for flow experiments and for immunofluorescence assays (Ibidi, $\mu$-Slide VI 0.5, glass bottom).

\subsubsection{Introducing principal component analysis and changepoint segmen- tation}

Principal Component Analysis (PCA), also known as Karhunen-Loeve transformation, is a statistical method for extracting patterns from complex data sets and reducing the dimension of complex signals. PCA involves transforming a set of data into a linear superposition of orthogonal components, arranged such that the first principal component has the largest possible variance; i.e., it accounts for the largest contribution to the variation in the data.

Therefore PCA is a fast and flexible unsupervised method for dimensionality reduction in data. Its behavior is most suitable to visualize by looking at a two-dimensional dataset like it is obtained by HVPT and shown in Figure 2.14 and 2.11. In this particular case, an application of PCA to the analysis of particle motion, the principal components' orthogonality gives PCA the ability to decompose the particle motion 
into its component frequencies.

We use an approach to search for changepoints, which is exact and under mild conditions has a linear computational cost in the number of data points [44]: the Pruned Exact Linear Time (PELT) method. This approach is based on the algorithm of Jackson et al. [34] but involves a pruning step within the dynamic program. This pruning reduces the computational cost of the method but does not affect the resulting segmentation's exactness. It can be applied to find changepoints under a range of statistical criteria such as penalized likelihood, quasi-likelihood [9], and cumulative sum of squares [33, 75].

It is implemented in a Python library for off-line change point detection called ruptures [92]. This package provides methods for the analysis and segmentation of nonstationary signals. Implemented algorithms include exact and approximate detection for various parametric and non-parametric models. Ruptures focus on ease of use by providing a well-documented and consistent interface.

From HVPT, we collect $\mathrm{x}-, \mathrm{y}^{-}$, and $\mathrm{z}$-positions. First, smoothing each trajectory with a Savitzky-Golay filter to average away particle localization errors, making a onedimensional distance axis. From this, we perform a Principal component analysis (PCA, Fig. 2.14) from sklearn.decomposition module for a linear dimensionality reduction using Singular Value Decomposition (SDV) to project it to a lower-dimensional space. Following a transformation of data into a linear superposition of orthogonal components, arranged such that the first principal component has the largest possible variance; i.e., it accounts for the largest contribution to the data variation. It is dividing the dataset into main and conjugated components for a chosen number of components of two. Each component is then analyzed by Pruned Exact Linear Time (PELT, Fig. 2.15) method to search for changepoints in the bead's motion pattern equivalent to binding and unbinding events of single tethers or of multiple tether formation aka interaction landscape (Fig. 2.14). PCA-Analysis-PELT-Segments are persistent for bond lifetimes. Afterward, each segment is characterized by its mean square displacements in two dimensions and its position fluctuation $\Delta x$. Confinement radii $r_{\text {conf }}$ and characteristic confinement time $\tau$ are then extracted by fitting $\left\langle r^{2}\right\rangle_{\text {confined }}=r_{\text {conf }}^{2} \cdot\left(1-e^{-\frac{t}{\tau}}\right)$ via non-linear least-squares minimization. The diffusion constant can be deducted from the characteristic time $\tau$. 

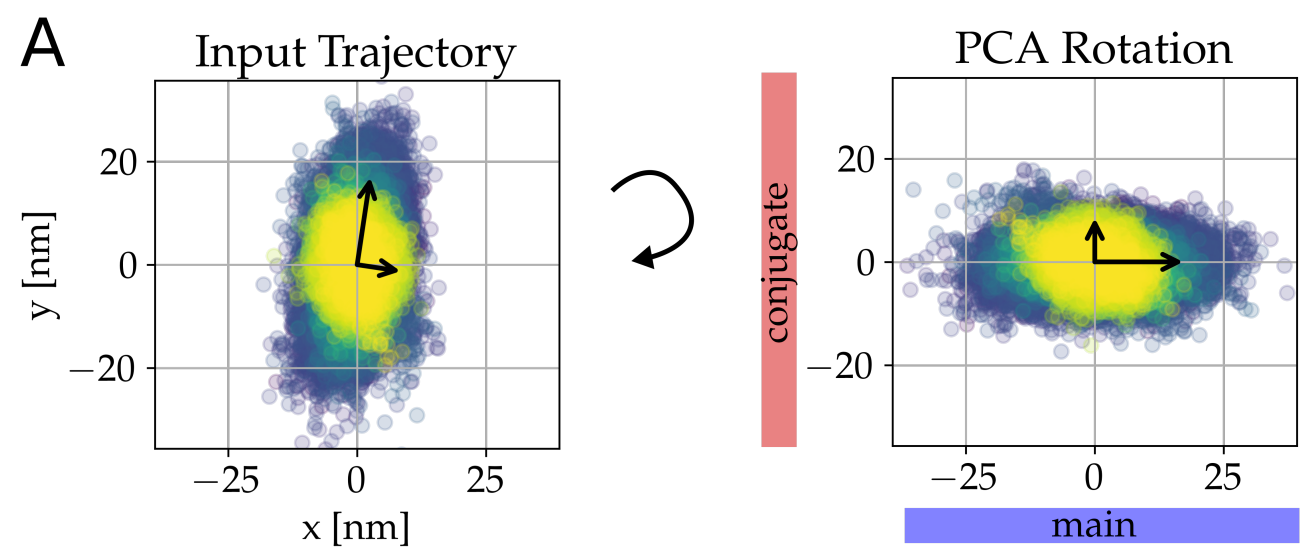

B
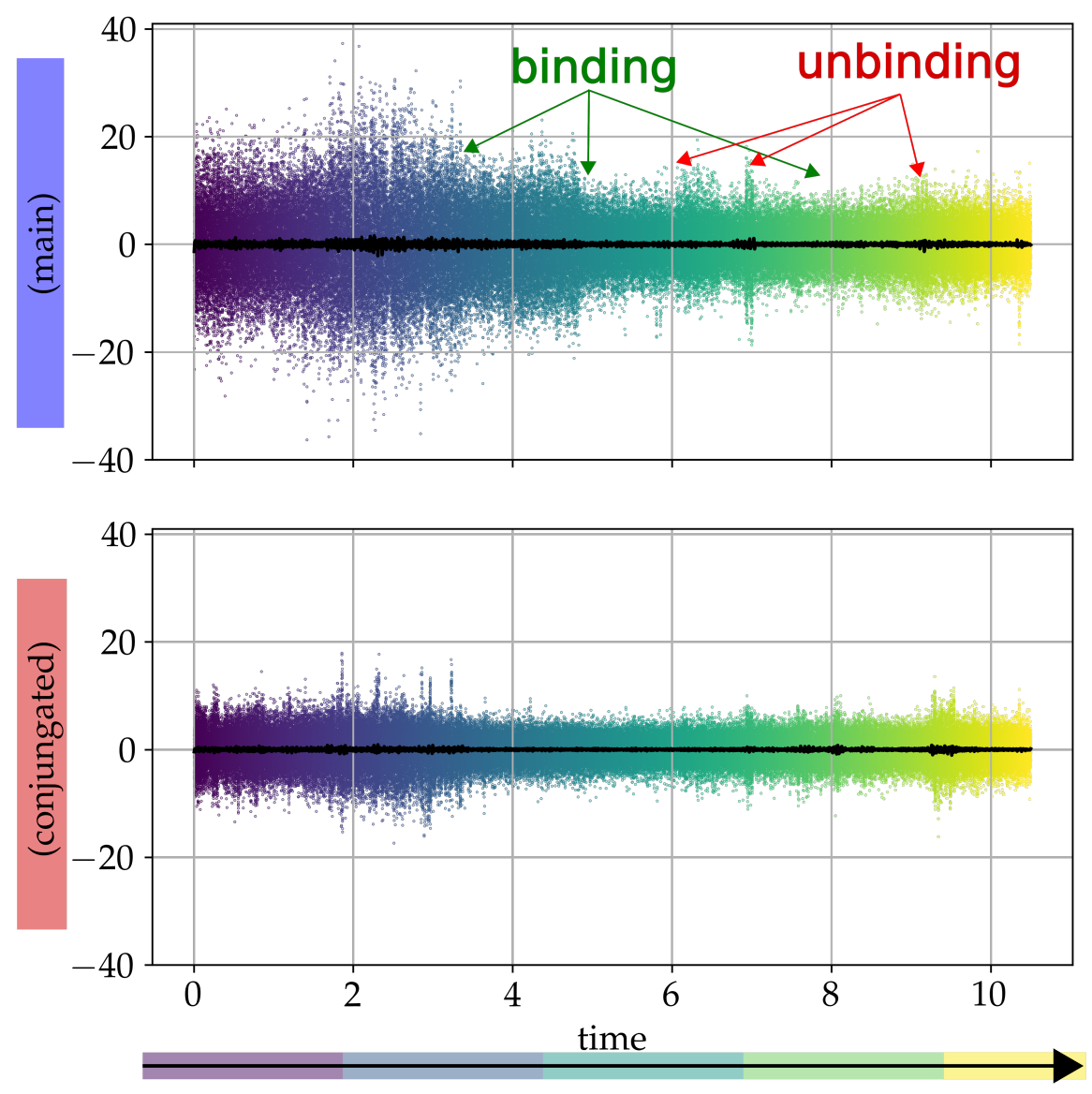

Figure 2.14: Application of PCA to the analysis of particle motion. A: trajectory position data is used as input for rotation into main and conjugate component axis. B: main and conjugate principal components are separated in their time courses. Both axis can be addressed individually for detection of binding or unbinding events via PELT. 

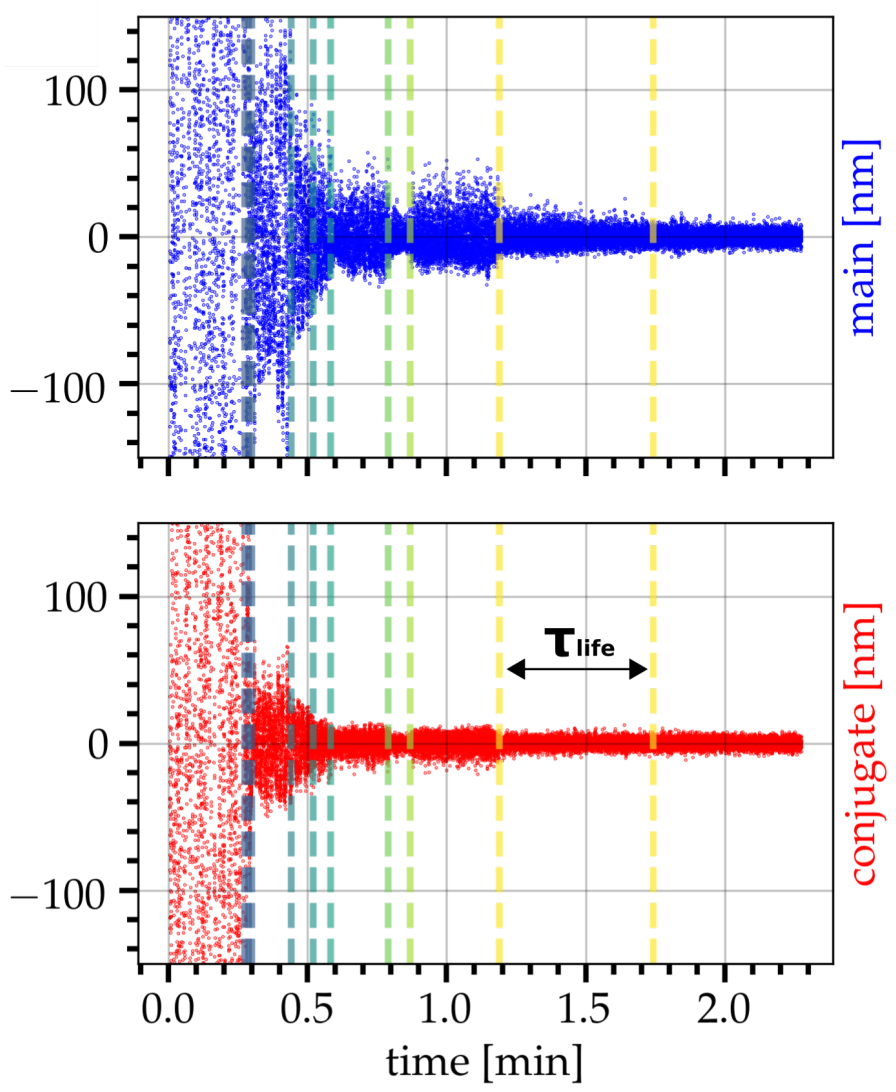

Figure 2.15: Illustration of a segmented tethered particle motion experiment. The PELT detection algorithm acting on experimental data after PCA. The experimental data provide the motion data as the position of the particle projected onto the $\mathrm{x}$ - or $\mathrm{y}$-plane parallel to the substrate. Motion corresponding to the position of the particle at different states as function of time from which a state lifetime $\tau_{\text {life }}$ can be extracted. 


\section{Reference}

[1] M. T. Abba and S. R. Kalidindi. Protocols for studying the time-dependent mechanical response of viscoelastic materials using spherical indentation stressstrain curves. Mechanics of Time-Dependent Materials, 2020. doi:10.1007/ s11043-020-09472-y.

[2] Daniel A. Alba Diz-Muñoz, A. Fletcher and D Orion Weiner. Use the force: Membrane tension as an organizer of cell shape and motility. Pesticide Biochemistry and Physiology, 2011. 100(2): 130-134. doi:10.1016/j.pestbp.2011.02.012.

[3] Bruce Alberts, Alexander Johnson, Julian Lewis, Peter Walter, Martin Raff, and Keith Roberts. Molecular Biology of the Cell 4th Edition: International Student Edition. Routledge, 2002. ISBN 9780815332886. Google-Books-ID: ozigkQEACAAJ.

[4] A. Ashkin. Acceleration and Trapping of Particles by Radiation Pressure. Physical Review Letters, 1970. 24(4): 156-159. doi:10.1103/PhysRevLett.24.156.

[5] Xavier Banquy, Kai Kristiansen, Dong Woog Lee, and Jacob N. Israelachvili. Adhesion and hemifusion of cytoplasmic myelin lipid membranes are highly dependent on the lipid composition. Biochimica et Biophysica Acta (BBA) - Biomembranes, 2012. 1818(3): 402-410. doi:10.1016/j.bbamem.2011.10.015.

[6] Solomon Barkley, Thomas G. Dimiduk, Jerome Fung, David M. Kaz, Vinothan N. Manoharan, Ryan McGorty, Rebecca W. Perry, and Anna Wang. Holographic Microscopy with Python and HoloPy. Computing in Science and Engineering, 2020. 22(5): 72-82. doi:10.1109/MCSE.2019.2923974.

[7] G. G. Bilodeau. Regular Pyramid Punch Problem. Journal of Applied Mechanics, 1992. 59(3): 519-523. doi:10.1115/1.2893754.

[8] Seth Blumberg, Arivalagan Gajraj, Matthew W. Pennington, and Jens-Christian Meiners. Three-Dimensional Characterization of Tethered Microspheres by Total Internal Reflection Fluorescence Microscopy. Biophysical Journal, 2005. 89(2): 1272-1281. doi:10.1529/biophysj.105.061242.

[9] Jerome V. Braun, Ruedi K. Braun, and Hans-Georg Müller. Multiple changepoint fitting via quasilikelihood, with application to dna sequence segmentation. Biometrika, 2000. 87: 301-314.

[10] F. Brochard-Wyart, N. Borghi, D. Cuvelier, and P. Nassoy. Hydrodynamic narrowing of tubes extruded from cells. Proceedings of the National Acade- 
my of Sciences of the United States of America, 2006. 103(20): 7660-7663. doi: $10.1073 /$ pnas.0602012103.

[11] Ann A.M. Bui, Anatolii V. Kashchuk, Marie Anne Balanant, Timo A. Nieminen, Halina Rubinsztein-Dunlop, and Alexander B. Stilgoe. Calibration of force detection for arbitrarily shaped particles in optical tweezers. Scientific Reports, 2018. 8(1): 1-12. doi:10.1038/s41598-018-28876-y.

[12] Hans Jürgen Butt, Brunero Cappella, and Michael Kappl. Force measurements with the atomic force microscope: Technique, interpretation and applications. Surface Science Reports, 2005. 59(1-6): 1-152. doi:10.1016/j.surfrep.2005.08.003.

[13] Marco A. Catipovic, Paul M. Tyler, Josef G. Trapani, and Ashley R. Carter. Improving the quantification of Brownian motion. American Journal of Physics, 2013. 81(7): 485-491. doi:10.1119/1.4803529.

[14] Andrea Mareike Cordes. Quantification of forces involved in carbohydrate induced attachment of carcinoma cells. .

[15] Paweł Czajka, Jan M. Antosiewicz, and Maclej Długosz. Effects of Hydrodynamic Interactions on the Near-Surface Diffusion of Spheroidal Molecules. ACS Omega, 2019. 4(16): 17016-17030. doi:10.1021/acsomega.9b02618.

[16] Emmanuel Detournay and Alexander H.-D. Cheng. Chapter 5: Fundamentals of Poroelasticity. Comprehensive Rock Engineering: Principles, Practice and Projects, Vol II: Analysis and Design Method, 1993. II: 113-171.

[17] Jan Domke and Manfred Radmacher. Measuring the elastic properties of thin polymer films with the atomic force microscope. Langmuir, 1998. 14(12): 33203325. doi:10.1021/la9713006.

[18] Mohammad Hadi Esteki, Ali Akbar Alemrajabi, Chloe M. Hall, Graham K. Sheridan, Mojtaba Azadi, and Emad Moeendarbary. A new framework for characterization of poroelastic materials using indentation. Acta Biomaterialia, 2020. 102: 138-148. doi:10.1016/j.actbio.2019.11.010.

[19] Jie Fang, Alexander Mehlich, Nobuyasu Koga, Jiqing Huang, Rie Koga, Xiaoye Gao, Chunguang Hu, Chi Jin, Matthias Rief, Juergen Kast, David Baker, and Hongbin Li. Forced protein unfolding leads to highly elastic and tough protein hydrogels. Nature Communications, 2013. 4. doi:10.1038/ncomms3974.

[20] Christian Ganser, Caterina Czibula, Daniel Tscharnuter, Thomas Schöberl, Chris- 
tian Teichert, and Ulrich Hirn. Combining adhesive contact mechanics with a viscoelastic material model to probe local material properties by AFM. Soft Matter, 2017. 14(1): 140-150. doi:10.1039/c7sm02057k.

[21] Yuval Garini, Guy Nir, and Moshe Lindner. Three dimensional tethered particle motion for dna-protein interactions studies. Biophysical Journal, 2012. 102(3): 386a. doi:10.1016/j.bpj.2011.11.2113.

[22] Nils C Gauthier, Olivier M Rossier, Anurag Mathur, James C Hone, and Michael P Sheetz. Plasma Membrane Area Increases with Spread Area by Exocytosis of a GPI-anchored Protein Compartment. Molecular biology of the cell, 2009. 20: 3261-3272.

[23] Emel I. Goksu, Juan M. Vanegas, Craig D. Blanchette, Wan-Chen Lin, and Marjorie L. Longo. AFM for structure and dynamics of biomembranes. Biochimica et Biophysica Acta (BBA) - Biomembranes, 2009. 1788(1): 254-266. doi: 10.1016/J.BBAMEM.2008.08.021.

[24] Jeff Gostick, Zohaib Khan, Thomas Tranter, Matthew Kok, Mehrez Agnaou, Mohammadamin Sadeghi, and Rhodri Jervis. PoreSpy: A Python Toolkit for Quantitative Analysis of Porous Media Images. Journal of Open Source Software, 2019. 4(37): 1296. doi:10.21105/joss.01296.

[25] Yesul Han, Jaeran Lee, Yumi Lee, and Sok Won Kim. Measurement of the Diffusion Coefficients of Fluorescence Beads and Quantum Dots by Using Fluorescence Correlation Spectroscopy. Journal of the Korean Physical Society, 2011. 59(5(1)): 3177-3181. doi:10.3938/jkps.59.3177.

[26] Alina Hategan, Richard Law, Samuel Kahn, and Dennis E. Discher. Adhesivelytensed cell membranes: Lysis kinetics and atomic force microscopy probing. Biophysical Journal, 2003. 85(4): 2746-2759. doi:10.1016/S0006-3495(03)74697-9.

[27] Fabian M. Hecht, Johannes Rheinlaender, Nicolas Schierbaum, Wolfgang H. Goldmann, Ben Fabry, and Tilman E. Schäffer. Imaging viscoelastic properties of live cells by AFM: power-law rheology on the nanoscale. Soft Matter, 2015. 11(23): 4584-4591. doi:10.1039/C4SM02718C.

[28] J. Manuel Hernández-Meza, J. R. Vélez-Cordero, Bernardo Yáñez-Soto, A. Ramírez-Saito, S. Aranda-Espinoza, and J. L. Arauz-Lara. Interaction of colloidal particles with biologically relevant complex surfaces. Colloids and Sur- 
faces A: Physicochemical and Engineering Aspects, 2019. 580(June): 123778. doi: 10.1016/j.colsurfa.2019.123778.

[29] R. M. Hochmuth and E. A. Evans. Extensional flow of erythrocyte membrane from cell body to elastic tether. I. Analysis. Biophysical Journal, 1982. 39(1): 71-81. doi:10.1016/S0006-3495(82)84492-5.

[30] Yuhang Hu and Zhigang Suo. Viscoelasticity and poroelasticity in elastomeric gels. Acta Mechanica Solida Sinica, 2012. 25(5): 441-458. doi:10.1016/S0894-9166(12) 60039-1.

[31] Yuhang Hu, Xuanhe Zhao, Joost J. Vlassak, and Zhigang Suo. Using indentation to characterize the poroelasticity of gels. Applied Physics Letters, 2010. 96(12): 121904. doi:10.1063/1.3370354.

[32] Jeffrey L. Hutter and John Bechhoefer. Calibration of atomic-force microscope tips. Review of Scientific Instruments, 1993. 64(7): 1868-1873. doi:10.1063/1.1143970.

[33] Carla Inclan and George C. Tiao. Use of cumulative sums of squares for retrospective detection of changes of variance. Journal of the American Statistical Association, 1994. 89: 913-923.

[34] Brad Jackson, Jeffrey D. Scargle, David Barnes, Sundararajan Arabhi, Alina Alt, Peter Gioumousis, Elyus Gwin, Paungkaew Sangtrakulcharoen, Linda Tan, and Tun Tao Tsai. An algorithm for optimal partitioning of data on an interval. IEEE Signal Processing Letters, 2005. 12(2): 105-108. doi:10.1109/LSP.2001.838216.

[35] Ingo Mey, Jan Rother, Helen Nöding and Andreas Janshoff. AFM-based microrheology reveals significant differences in the viscoelastic response between malign and benign cell lines. Nature Chemistry, 2014. 6(3): 1-23. doi: 10.1038/NCHEM.1850.

[36] Yuekan Jiao and Tilman E. Schäffer. Accurate height and volume measurements on soft samples with the atomic force microscope. Langmuir, 2004. 20(23): 1003810045. doi:10.1021/la048650u. PMID: 15518491.

[37] Alexandr Jonáš, Martin Kochanczyk, Alexandro D. Ramirez, Michael Speidel, and Ernst Ludwig Florin. Mechanical Contact Spectroscopy: Characterizing Nanoscale Adhesive Contacts via Thermal Forces. Langmuir, 2019. 35(17): 58095820. doi:10.1021/acs.langmuir.8b04074. 
[38] Thomas Jue. Fundamental Concepts in Biophysics: Volume 1. Humana Press, 2010. ISBN 9781617378614. Google-Books-ID: 1lECfAEACAAJ.

[39] Thomas Jue, Subhash H. Risbud, Marjorie L. Longo, and Roland Faller, editors. Biomembrane Frontiers: Nanostructures, Models, and the Design of Life. Handbook of Modern Biophysics. Humana Press, 2009. ISBN 9781607613138. doi:10.1007/ 978-1-60761-314-5.

[40] J.W. Dai and M.P. Sheetz. Membrane tether formation from blebbing cells. Biophysical journal, 1999. 77(6): 3363-3370.

[41] Douwe Kamsma. Acoustic Force Spectroscopy (AFS) From single molecules to single cells. 2018. ISBN 9789402810097.

[42] Douwe Kamsma, Ramon Creyghton, Gerrit Sitters, Gijs J.L. Wuite, and Erwin J.G. Peterman. Tuning the Music: Acoustic Force Spectroscopy (AFS) 2.0. Methods, 2016. 105: 26-33. doi:10.1016/j.ymeth.2016.05.002.

[43] Andrea Keidel, Tobias F. Bartsch, and Ernst Ludwig Florin. Direct observation of intermediate states in model membrane fusion. Scientific Reports, 2016. 6(March): 1-11. doi:10.1038/srep23691.

[44] R. Killick, P. Fearnhead, and I. A. Eckley. Optimal detection of changepoints with a linear computational cost. Journal of the American Statistical Association, 2012. 107(500): 1590-1598. doi:10.1080/01621459.2012.737745.

[45] Julie C. Kohn and Donna M. Ebenstein. Eliminating adhesion errors in nanoindentation of compliant polymers and hydrogels. Journal of the Mechanical Behavior of Biomedical Materials, 2013. 20: 316-326. doi:10.1016/j.jmbbm.2013.02.002.

[46] Stylianos-Vasileios Kontomaris*. The Hertz Model in AFM Nanoindentation Experiments: Applications in Biological Samples and Biomaterials, 2018.

[47] L. Kou, L. Jin, H. Lei, C. Hu, H. Li, X. Hu, and X. Hu. Real-time parallel 3D multiple particle tracking with single molecule centrifugal force microscopy. Journal of Microscopy, 2019. 273(3): 178-188. doi:10.1111/jmi.12773.

[48] Daniel T. Kovari, Eric Weeks, David Dunlap, and Laura Finzi. Tuning up tethered particle motion. Biophysical Journal, 2017. 112(3): 70a. doi:10.1016/j.bpj.2016.11. 421.

[49] Sandip Kumar, Carlo Manzo, Chiara Zurla, Suleyman Ucuncuoglu, Laura Finzi, 
and David Dunlap. Enhanced tethered-particle motion analysis reveals viscous effects. Biophysical Journal, 2014. 106(2): 399-409. doi:10.1016/j.bpj.2013.11.4501.

[50] Norbert Kučerka, Stephanie Tristram-Nagle, and John F. Nagle. Structure of Fully Hydrated Fluid Phase Lipid Bilayers with Monounsaturated Chains. The Journal of Membrane Biology, 2006. 208(3): 193-202. doi:10.1007/s00232-005-7006-8.

[51] Yang Lai and Yuhang Hu. Unified solution for poroelastic oscillation indentation on gels for spherical, conical and cylindrical indenters. Soft Matter, 2017. 13(4): 852-861. doi:10.1039/c6sm02341j.

[52] L.D. Landau, E.M. Lifshitz, A.M. Kosevich, J.B. Sykes, L.P. Pitaevskii, and W.H. Reid. Theory of Elasticity: Volume 7. Course of theoretical physics. Elsevier Science, 1986. ISBN 9780750626330.

[53] Sang-Hyuk Lee, Yohai Roichman, Gi-Ra Yi, Shin-Hyun Kim, Seung-Man Yang, Alfons van Blaaderen, Peter van Oostrum, and David G. Grier. Characterizing and tracking single colloidal particles with video holographic microscopy. Optics Express, 2007. 15(26): 18275. doi:10.1364/oe.15.018275.

[54] Matthew Leivers, John M. Seddon, Marc Declercq, Eric Robles, and Paul Luckham. Measurement of Forces between Supported Cationic Bilayers by Colloid Probe Atomic Force Microscopy: Electrolyte Concentration and Composition. Langmuir, 2019. 35(3): 729-738. doi:10.1021/acs.langmuir.8b03555.

[55] Tongcang Li and Mark G. Raizen. Brownian motion at short time scales. Annalen der Physik, 2013. 525(4): 281-295. doi:10.1002/andp.201200232.

[56] Reinhard Lipowsky. Coupling of bending and stretching deformations in vesicle membranes. Advances in Colloid and Interface Science, 2014. 208: 14-24. doi: 10.1016/j.cis.2014.02.008.

[57] Enrique A. López-Guerra, Babak Eslami, and Santiago D. Solares. Calculation of standard viscoelastic responses with multiple retardation times through analysis of static force spectroscopy AFM data. Journal of Polymer Science, Part B: Polymer Physics, 2017. 55(10): 804-813. doi:10.1002/polb.24327.

[58] Bärbel Lorenz, Rabea Keller, Eva Sunnick, Burkhard Geil, and Andreas Janshoff. Colloidal probe microscopy of membrane-membrane interactions: From ligand-receptor recognition to fusion events. Biophysical Chemistry, 2010. 150(1): 54-63. doi:10.1016/j.bpc.2010.02.008. 
[59] Lumicks. G2 AFS Customer Hardware Guide.

[60] Lumicks. User Guide Acoustic Force Spectroscope.

[61] Andrea Malandrino and Emad Moeendarbary. Poroelasticity of Living Tissues. Elsevier Inc., 2017. ISBN 9780128012383. doi:10.1016/b978-0-12-801238-3.99932-x.

[62] Manoel Manghi, Nicolas Destainville, and Annaël Brunet. Statistical physics and mesoscopic modeling to interpret tethered particle motion experiments. Methods, 2019. 169: 57-68. doi:10.1016/j.ymeth.2019.07.006.

[63] Koen E. Merkus, Menno W.J. Prins, and Cornelis Storm. Single-Bond Association Kinetics Determined by Tethered Particle Motion: Concept and Simulations. Biophysical Journal, 2016. 111(8): 1612-1620. doi:10.1016/j.bpj.2016.08.045.

[64] Mario M. Modena, Bastian Rühle, Thomas P. Burg, and Stefan Wuttke. Nanoparticle Characterization: What to Measure? Advanced Materials, 2019. 31(32): 1-26. doi:10.1002/adma.201901556.

[65] Emad Moeendarbary. Time-dependent mechanics of living cells.

[66] Emad Moeendarbary, Léo Valon, Marco Fritzsche, Andrew R. Harris, Dale A. Moulding, Adrian J. Thrasher, Eleanor Stride, L. Mahadevan, and Guillaume T. Charras. The cytoplasm of living cells behaves as a poroelastic material. Nature Materials, 2013. 12(3): 253-261. doi:10.1038/nmat3517.

[67] Anne Mongruel. Near-wall hydrodynamic interactions between a settling sphere and a wall. Journal of Physics: Conference Series, 2012. 392(1). doi: 10.1088/1742-6596/392/1/012011.

[68] C.E. Morris and U. Homann. Cell Surface Area Regulation and Membrane Tension. The Journal of Membrane Biology, 2001. 179(2): 79-102. doi:10.1007/ s002320010040.

[69] V. C. Mow, S. C. Kuei, W. M. Lai, and C. G. Armstrong. Biphasic Creep and Stress Relaxation of Articular Cartilage in Compression: Theory and Experiments. Journal of Biomechanical Engineering, 1980. 102(1): 73. doi:10.1115/1.3138202.

[70] KC Keir C Neuman and Attila Nagy. Single-molecule force spectroscopy: optical tweezers, magnetic tweezers and atomic force microscopy. Nature methods, 2008. 5(6): 491-505. doi:10.1038/nmeth.1218.Single-molecule.

[71] Marieelen Oelkers, Hannes Witt, Partho Halder, Reinhard Jahn, and Andreas 
Janshoff. SNARE-mediated membrane fusion trajectories derived from forceclamp experiments. Proceedings of the National Academy of Sciences, 2016. 113(46): 13051-13056. doi:10.1073/pnas.1615885113.

[72] M. L. Oyen. Mechanical characterisation of hydrogel materials. International Materials Reviews, 2014. 59(1): 44-59. doi:10.1179/1743280413Y.0000000022.

[73] Michelle L Oyen. Poroelastic nanoindentation responses of hydrated bone. Journal of Materials Research, 2018. 23(5): 1307-1314. doi:10.1557/JMR.2008.0156.

[74] $\dagger$ Philip C. Nelson, $\ddagger$ Chiara Zurla, $\ddagger$ Doriano Brogioli, $†$ John F. Beausang, § Laura Finzi, , and || David Dunlap*. Tethered Particle Motion as a Diagnostic of DNA Tether Length, 2006. doi:10.1021/jp0630673.

[75] Franck Picard, Emilie Lebarbier, Mark Hoebeke, Guillem Rigaill, Baba Thiam, and Stéphane Robin. Joint segmentation, calling, and normalization of multiple CGH profiles. Biostatistics, 2011. 12(3): 413-428. doi:10.1093/biostatistics/kxq076.

[76] Anna Pietuch, Bastian R. Brückner, and Andreas Janshoff. Membrane tension homeostasis of epithelial cells through surface area regulation in response to osmotic stress. Biochimica et Biophysica Acta (BBA) - Molecular Cell Research, 2013. 1833(3): 712-722. doi:https://doi.org/10.1016/j.bbamcr.2012.11.006.

[77] Anna Pietuch, Bastian Rouven Brückner, Tamir Fine, Ingo Mey, and Andreas Janshoff. Elastic properties of cells in the context of confluent cell monolayers: impact of tension and surface area regulation. Soft Matter, 2013. 9(48): 1149011502. doi:10.1039/C3SM51610E.

[78] W Rawicz, K C Olbrich, T McIntosh, D Needham, and E Evans. Effect of Chain Length and Unsaturation on Elasticity of Lipid Bilayers. Biophysical Journal, 2000. 79(1): 328-339.

[79] Maurizio Righini, Antony Lee, Cristhian Cañari-Chumpitaz, Troy Lionberger, Ronen Gabizon, Yves Coello, Ignacio Tinoco, and Carlos Bustamante. Full molecular trajectories of rna polymerase at single base-pair resolution. Proceedings of the $\mathrm{Na}$ tional Academy of Sciences, 2018. 115(6): 1286-1291. doi:10.1073/pnas.1719906115.

[80] Nadia Ruthardt, Don C. Lamb, and Christoph Bräuchle. Single-particle tracking as a quantitative microscopy-based approach to unravel cell entry mechanisms of viruses and pharmaceutical nanoparticles. Molecular Therapy, 2011. 19(7): 1199-1211. doi:10.1038/mt.2011.102. 
[81] Matthias Schickinger and Hendrik Dietz. Dna-origami-based tethered particle motion for the single-molecule study of bimolecular interactions. Biophysical Journal, 2017. 112(3): 301a. doi:10.1016/j.bpj.2016.11.1627.

[82] Kai D Schleicher. Reduction of Dimensionality in Karyopherin Mediated Transport on FG Domains. 2016.

[83] Alastair Phillip Selby. Nanoindentation of soft contact lens materials. 2012.

[84] A. P. S. Selvadurai, editor. Mechanics of Poroelastic Media, volume 35. Springer Netherlands, 1996. ISBN 978-90-481-4513-3. doi:10.1007/978-94-015-8698-6.

[85] Shamik Sen, Shyamsundar Subramanian, and Dennis E. Discher. Indentation and Adhesive Probing of a Cell Membrane with AFM: Theoretical Model and Experiments. Biophysical Journal, 2005. 89(5): 3203-3213. doi:10.1529/biophysj. 105.063826.

[86] Michael P. Sheetz. Cell control by membrane-cytoskeleton adhesion. Nature Reviews Molecular Cell Biology, 2001. 2(5): 392-396. doi:10.1038/35073095.

[87] Ian N. Sneddon. The relation between load and penetration in the axisymmetric boussinesq problem for a punch of arbitrary profile. International Journal of Engineering Science, 1965. 3(1): 47-57. doi:https://doi.org/10.1016/0020-7225(65) 90019-4.

[88] Daniel G T Strange, Timothy L. Fletcher, Khaow Tonsomboon, Helen Brawn, Xuanhe Zhao, and Michelle L. Oyen. Separating poroviscoelastic deformation mechanisms in hydrogels. Applied Physics Letters, 2013. 102(3): 3-7. doi:10.1063/ 1.4789368 .

[89] Fidan Sumbul, Nahid Hassanpour, Jorge Rodriguez-Ramos, and Felix Rico. OneStep Calibration of AFM in Liquid. Frontiers in Physics, 2020. 8(September): 1-10. doi:10.3389/fphy.2020.00301.

[90] Aartjan J.W. Te Velthuis, Jacob W.J. Kerssemakers, Jan Lipfert, and Nynke H. Dekker. Quantitative guidelines for force calibration through spectral analysis of magnetic tweezers data. Biophysical Journal, 2010. 99(4): 1292-1302. doi: 10.1016/j.bpj.2010.06.008.

[91] Simon F. Tolić-Nørrelykke, Erik Schäffer, Jonathon Howard, Francesco S. Pavone, Frank Jülicher, and Henrik Flyvbjerg. Calibration of optical tweezers with 
positional detection in the back focal plane. Review of Scientific Instruments, 2006. 77(10): 103101. doi:10.1063/1.2356852.

[92] Charles Truong, Laurent Oudre, and Nicolas Vayatis. Selective review of offline change point detection methods. Signal Processing, 2020. 167: 107299. doi: 10.1016/j.sigpro.2019.107299.

[93] Marijn T.J. Van Loenhout, Jacob W.J. Kerssemakers, Iwijn De Vlaminck, and Cees Dekker. Non-bias-limited tracking of spherical particles, enabling nanometer resolution at low magnification. Biophysical Journal, 2012. 102(10): 2362-2371.

[94] Emiel W.A. Visser, Junhong Yan, Leo J. Van IJzendoorn, and Menno W.J. Prins. Continuous biomarker monitoring by particle mobility sensing with single molecule resolution. Nature Communications, 2018. 9(1): 1-10. doi: $10.1038 / \mathrm{s} 41467-018-04802-8$.

[95] Giorgio Volpe, Sylvain Gigan, and Giovanni Volpe. Simulation of the active Brownian motion of a microswimmer. American Journal of Physics, 2014. 82(7): 659-664. doi:10.1119/1.4870398.

[96] Jie Wei, Barbara L. McFarlin, and Amy J. Wagoner Johnson. A multi-indent approach to detect the surface of soft materials during nanoindentation. Journal of Materials Research, 2016. 31(17): 2672-2685. doi:10.1557/jmr.2016.265.

[97] Hannes Witt and Andreas Janshoff. Using Force Spectroscopy to Probe CoiledCoil Assembly and Membrane Fusion. Springer New York, New York, NY. ISBN 978-1-4939-8760-3, 2019. 145-159. doi:10.1007/978-1-4939-8760-3_8.

[98] Ewa P. Wojcikiewicz, Xiaohui F. Zhang, and Vincent T. Moy. Force and compliance measurements on living cells using atomic force microscopy (afm). Biological Procedures Online, 2008. 6:1-9.

[99] J L Wuite. Manual for AFS particle tracking and data analysis software. Lumicks B V Manual, 2018: 1-22.

[100] Goh Haw Zan, Cheemeng Tan, Markus Deserno, Frederick Lanni, and Mathias Lösche. Hemifusion of giant unilamellar vesicles with planar hydrophobic surfaces: a fluorescence microscopy study. Soft Matter, 2012. 8(42): 10877-10886. doi:10.1039/C2SM25702E.

[101] Joanna Zemła, Justyna Bobrowska, Andrzej Kubiak, Tomasz Zieliński, Joanna Pabijan, Katarzyna Pogoda, Piotr Bobrowski, and Małgorzata Lekka. Indenting 
soft samples (hydrogels and cells) with cantilevers possessing various shapes of probing tip. European Biophysics Journal, 2020. 49(6): 485-495. doi:10.1007/ s00249-020-01456-7.

[102] Hanqing Zhang, Tim Stangner, Krister Wiklund, Alvaro Rodriguez, and Magnus Andersson. UmUTracker: A versatile MATLAB program for automated particle tracking of 2D light microscopy or 3D digital holography data. Computer Physics Communications, 2017. 219: 390-399. doi:10.1016/j.cpc.2017.05.029. 


\section{CHAPTER 3}

\section{BIOPHYSICAL MATERIALS AND TECHNIQUES}

In the following chapter, all chemicals used in this work, such as buffers, lipids, proteins, detergents, and fluorescent dyes, materials used, such as PDMS and glass beads, as well as all preparative and analytical methods used for evaluation are presented.

\subsection{Buffers, chemicals and detergents}

All chemicals used were obtained from commercial suppliers (Sigma Aldrich, Avanti Polar Lipids, GE Healthcare Life Sciences, etc.) and used without further purification. All experiments and purification by column chromatography were carried out with freshly prepared buffers which had previously been degassed and filtered under reduced pressure.

\subsubsection{Buffers}

The buffer systems used in this work and listed in Table 3.1.1 were prepared with ultra-pure water from MilliQ Systems (EMD-Millipore, Merck Darmstadt, Germany) and, filtered (cellulose acetate filter, $0.2 \mu \mathrm{m}$ pore size) and degassed for about $2.5 \mathrm{~h}$ under reduced pressure and constant stirring. All buffers were adjusted to a $\mathrm{pH}$ of 7.4. Suspended solids were removed by filtering them through a cellulose acetate membrane (Minisart, Satorius, Göttingen, Germany). The osmolarity of the buffer was determined with an osmometer (Osmomat 3000, Gonotec, Berlin, Germany).

\subsubsection{Lipids}

Each type of cell membrane has its characteristic lipid composition, which differs in the type of fatty acid chains and the nature of the head groups. For the investigation 


\begin{tabular}{|c|c|c|c|}
\hline Label & Composition & $\mathrm{pH}$ or $\mathrm{T}$ & Usage \\
\hline HEP100A & Hepes $20 \mathrm{mM}, \mathrm{KCl} 100 \mathrm{mM}$ & 7.4 & colloidal probe assay \\
\hline HEP100B & Hepes $20 \mathrm{mM}, \mathrm{KCl} 100 \mathrm{mM}$, EDTA $1 \mathrm{mM}$, DTT 1mM & -"- & $v$ - und $t$-SNARE \\
\hline HEP300SP & Hepes $10 \mathrm{mM}, \mathrm{NaCl} 300 \mathrm{mM}$ & -"- & membrane coated beads \\
\hline HEP150 & Hepes $150 \mathrm{mM}, \mathrm{KCl} 150 \mathrm{mM}$, EGTA $1 \mathrm{mM}$, DTT $1 \mathrm{mM}$ & -"- & Syt-1 w/o Calcium, AFM \\
\hline HEP150-Ca & Hepes $150 \mathrm{mM}, \mathrm{KCl} 150 \mathrm{mM}, \mathrm{CaCl}_{2} 0.1 \mathrm{mM}$, DTT1mM & -"- & Syt-1 w/ Calcium, AFM \\
\hline $\mathrm{NaC} 20$ & Na-Citrate $20 \mathrm{mM}, \mathrm{KCl} 50 \mathrm{mM}, \mathrm{NaN}_{3} 0.1 \mathrm{mM}$ & 4.8 & membrane spreading \\
\hline HEP100 & Hepes $20 \mathrm{mM}, \mathrm{KCl} 100 \mathrm{mM}$, EGTA $1 \mathrm{mM}$, DTT $1 \mathrm{mM}$ & 7.4 & Syt-1 w/o Calcium, HVPT \\
\hline HEP100-Ca & Hepes $20 \mathrm{mM}, \mathrm{KCl} 100 \mathrm{mM}, \mathrm{CaCl}_{2} 0.1 \mathrm{mM}$, DTT $1 \mathrm{mM}$ & -"- & Syt-1 w/ Calcium, HVPT \\
\hline FG-SAB & Tris/HCl $20 \mathrm{mM}, \mathrm{NaCl} 130 \mathrm{mM}, \mathrm{MgCl}_{2} 2 \mathrm{mM}, \pm$ DTT $1 \mathrm{mM}$ & 7.5 & FG AFM indentations experiments \\
\hline FG-PBS & Phosphate buffered saline, $\mathrm{NaCl} 137 \mathrm{mM}, \mathrm{KCl} 2.68 \mathrm{mM}, \mathrm{Na}_{2} \mathrm{HPO}_{4} 1.47 \mathrm{mM}$ & 7.4 & FG Hydrogel poroelastic relaxation \\
\hline GM & $450 \mathrm{~mL}$ DMEM, $4.5 \mathrm{~g} / \mathrm{L}$ Glucose with L-Glutamin $4 \mathrm{mM}, 50 \mathrm{~mL}$ FCS Fetal Bovine Serum & $37^{\circ} \mathrm{C} 7.5 \% \mathrm{CO}_{2}$ & growth medium $\mathrm{C} 2 \mathrm{C} 12$ cell line \\
\hline DM & DMEM containing $5 \%$ heat-inactivated horse serum $(\mathrm{HS})+$ antibiotics & -"- & differentiation medium $\mathrm{C} 2 \mathrm{C} 12$ cell line \\
\hline
\end{tabular}

Table 3.1: Overview of the buffers used and their composition.

of biological membranes in vitro, different types of model membrane systems have been developed mimicking the natural composition of cellular plasma membranes. The composition of lipids used in this work is based on the natural lipid composition of synaptic vesicles with $40 \%$ phosphatidylcholines, $32 \%$ phosphatidylethanolamines, $12 \%$ phosphatidylserines, $5 \%$ phosphatidylinositol and 10\% cholesterol $w t / w t$. [4] In order to achieve a higher fluidity of the lipids at room temperature, the following 1,2-dioleyl-phosphatidyls (18:1) were used instead of 1-palmityl-2-oleyl-phosphatidyls $(16: 0,18: 1)$ in a mass ratio of 55:22:11:11:1 [32].

1,2-Dioleoyl-sn-glycero-3-phosphocholin (DOPC) with a phase transition temperature from $T_{M}=-17^{\circ} \mathrm{C}$ is a fluid ionic lipid from the group of phosphatidylcholines (PC). [32] It is almost cylindrical and has a negative curvature. [6]<smiles>CCCCCC/C=C\CCCCCCCCC(=O)O[C@H](COC(=O)CCCCCCCC/C=C\CCCCCCCC)COP(=O)(O)OCC[N+](C)(C)C</smiles>

1,2-Dioleoyl-sn-glycero-3-phosphoethanolamine (DOPE) with a phase transition temperature of $T_{M}=-16^{\circ} \mathrm{C}$ is a phosphatidylethanolamine (PE), which can be labeled via the amino group at the end of the alkyl residue with fluorescent dyes (ATTO label). PE lipids have a strong negative curvature and can stabilize the stalk intermediate and increase the rate of fusion. [21, 25]<smiles></smiles>

1,2-Dioleoyl-sn-glycero-3-phosphoserin (DOPS) with a phase transition temperature of 
$T_{M}=-11^{\circ} \mathrm{C}$ is a phosphatidylserin (PS). Due to their small head group in proportion to the hydrophobic side chain, they can stabilize the fusion-stalk. [3]<smiles>CCCCCC/C=C\CCCCCCCC(=O)O[C@H](COC(=O)CCCCCCCC/C=C\CCCCCCCC)COP(=O)(O)OCC(N)C(=O)O</smiles>

Cholesterol (Chol) is a particularly readily soluble component in lipids that ensures the stability of the membrane especially at physiological temperatures. [14, 22] Also, cholesterol binds to the vesicular protein synaptophysin and thus modulates the interaction with synaptobrevin on the active Zones at the synaptic cleft. [16, 31]

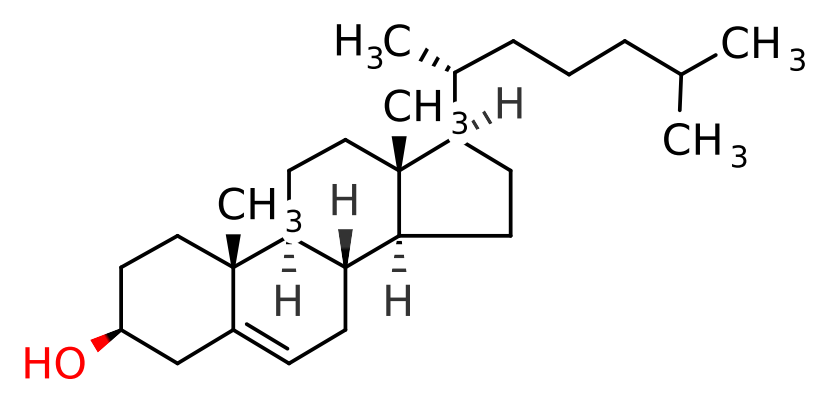

1,2-Dihexadecyl-sn-glycerophosphoethanolamine is a synthetic phosphatidylethanolamine (PE), which connects to the dye Texas $\operatorname{Red}^{\circledR}(\mathrm{TR})$ via the amino group at the end of the alkyl residue and is used as a fluorescent probe. [11]<smiles>CCCCCCCCCCCCCCCCCCO[C@H](COCCCCCCCCCCCCCCC)COP(=O)(O)OCCN</smiles>

\subsubsection{Proteine}

\section{Ectodomain of myomerger}

For investigation of tension generation during Myoblast membrane fusion in model systems, a ectodomain of full-length Myomerger was used ${ }^{1}$. Generated recombinant

\footnotetext{
${ }^{1}$ Frozen aliquots of the Myomerger Ectodomain was kindly provided by the Lab of Dr. Leonid Chernomordik.
} 
proteins using either $p M A L X$ or a modified $p E T 28 a$ backbone were provided. Each of these plasmids contains maltose-binding protein (MBP) as an N-terminal fusion protein. However, the modified pET28a contains an N-terminal 6x histidine tag, thrombin cleavage site, and a PreScission Protease (PP) cleavage site after MBP. They cloned full-length Myomerger into pET28a-His-thrombin-MBP-PP and the ectodomain of Myomerger (amino acids 26-84) into both plasmids. The cDNA encoding full-length Myomerger or its ectodomain was obtained by amplifying the region from full-length Myomerger as a template [17].

\section{Recombinant SNARE proteins [10]:}

Sequences of recombinant SNARE proteins from the genus Rattus norvegicus were reconstituted in unilamellar vesicles to mediate the fusion process ${ }^{1}$. This involved the sequence for the protein Synaptobrevin-1-116 (M=12.691 kDa) and for the stabilized $\Delta \mathrm{N} 49$ acceptor complex $(\mathrm{M}=40.963 \mathrm{kDa})$ from Syntaxin-1A, SNAP-25th and f-Syt-1

The t-SNARE SNAP25a is anchored in the presynaptic membrane via four palmitoyl linkers, post-translationally connected to cysteines at positions 85, 88, 90, and 92 in the amino acid sequence. For exclusion of side reactions during heterologous expression of SNAP25a in E. coli and prevention of cross-linking, a mutant was used in which the four cysteines were replaced by alanine. SNAP25a has a total length of 206 amino acids. It exhibits two $\alpha$-helical parts (residues 7-82 and residues 142-201) harboring the two SNARE-motifs involved in the formation of the parallel four-helix SNARE-bundle.

The t-SNARE syntaxin-1A (syx-1A) was used in a shortened version, lacking the Nterminal Habc-domain, which constitutes a substantial part of the cytosolic region of the protein. The $\mathrm{H}_{\mathrm{abc}}$-domain was shown to regulate the opening and closing of the t-SNARE under physiological conditions. To gather control over open and closed states, regulatory factors and proteins like munc-18 are needed. So, for simplification and enhancement of fusogenic in our model system, the mutant syx-1A (residues 183-288) was used, comprising the SNARE-motif (residues 192-254) and the transmembrane domain (TMD, residues 266-288) as central parts of the protein.

Synaptobrevin 2 (residues 1-116, syb2), also referred to as full-length syb2, is a SNAREprotein localized in vivo in the membrane of synaptic vesicles and, therefore, belongs to the class of v-SNAREs. Syb 2 exhibits a C-terminal transmembrane domain (TMD, residues 95-114) with $\alpha$-helical character serving as a membrane anchor. The SNAREmotif (residues 31-91), also showing an $\alpha$-helical secondary structure is connected to the

\footnotetext{
${ }^{1}$ Frozen aliquots for reconstitution of SNAREs and Synaptotagmin were kindly provided by the Lab of Prof. Dr. Claudia Steinem and the Lab of Prof. Dr. Reinhard Jahn.
} 
TMD via a short linker region. Besides the full-length syb2, also shortened versions of the protein were used. So, for the formation of the stabilized t-SNARE acceptor complex $\triangle \mathrm{N} 49$, the fragment syb2 (residues 49-96) was needed. For control experiments to verify SNARE specificity, the water-soluble fragment syb2 (residues 1-96) carrying the full SNARE-motif was utilized.

Synaptotagmin-1 (residues 1-421, syt-1) is one of the leading candidates as a $\mathrm{Ca}^{2+}$ sensor in neuronal signal transmission. It is comprised of a short and unstructured intravesicular part (residues 1-57) at the N-terminus, followed by a single $\alpha$-helical transmembrane domain (TMD, residues 58-79) and a cytosolic part (residues 80-421). An unstructured linker region in the cytosolic part (residues 80-142) connects the TMD with the two $\mathrm{Ca}^{2+}$ binding domains C2A (residues 143-244) and C2B (residues 274-377).

\section{Nucleoporin 98 and nuclear transport factor 2}

The NPC barrier in Xenopus relies primarily on the intrinsically disordered FG domain of Nup98. All Nup98 FG phases rejected inert macromolecules and yet allowed far larger NTR cargo complexes to rapidly enter ${ }^{1}$.

FG domains were purified using $\mathrm{Ni}(\mathrm{II})$ chelate chromatography under denaturing conditions (100 mMTris pH 8, $8 \mathrm{M} \mathrm{GuaHCl}, 10 \mathrm{mM}$ DTT). Elution was with imidazole

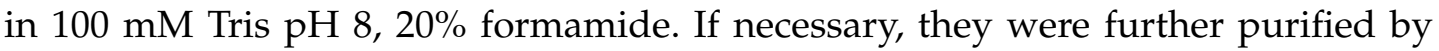
covalent chromatography. An engineered C-terminal cysteine was allowed to form a disulfide bond with a 2-thiopyridine-activated $\mathrm{SH}$-silica matrix, and elution was achieved by reducing the disulfides with DTT. FG domains were re-buffered to $20 \%$ acetonitrile, $0.08 \%$ TFA, and lyophilized.

All NTRs, transport substrates and inert molecules were purified under $\mathrm{N}$-terminal His-tags and native $\mathrm{Ni}$ (II) chelate chromatography. Elution was performed with either imidazole or by on-column protease cleavage [8]. The tags of all imidazole-eluted proteins were cleaved off in solution with TEV protease, and proteins were further purified by gel filtration on a Superdex 200 column equilibrated with $44 \mathrm{mM}$ Tris $\mathrm{pH}$ 7.5, $290 \mathrm{mM} \mathrm{NaCl}, 4.4 \mathrm{mM} \mathrm{MgCl}_{2}, 5 \mathrm{mM} \mathrm{DTT}$, and eventually snap-frozen in liquid nitrogen after addition of $250 \mathrm{mM}$ sucrose [15].

\footnotetext{
${ }^{1}$ Both were kindly provided by Sheung Chun Ng and the Lab of Prof. Dr. Dirk Görlich, Max Planck Institute, Göttingen.
} 


\subsubsection{Detergents}

$n$-Octyl- $\beta$-D-glucoside is a detergent molecule mainly used in the reconstitution of integral membrane proteins into lipid membranes. It promotes lipid detergent micelles above its critical micelle concentration, which completely dissolves the proteins without denaturing them. [13, 20, 36]<smiles>CCCCCCCCO[C@H]1O[C@H](CO)[C@@H](O)[C@H](O)[C@H]1O</smiles>

\subsubsection{Fluorescent dyes}

Because of the complexity of living cells, protein-mediated in vitro membrane fusion assays are a successful alternative approach to a molecular-level understanding of cell-based assays. Many artificial model systems and methods have been set up so far to investigate the mechanism of membrane fusion. These models are primarily based on liposomes, consisting of different lipids, fluorophores, and are usually composed of one or two fusogenic. Fluorescence spectroscopy is widely used to research such models in bulk phase solution. From the change in fluorescence intensity, lipid mixing and material mixing are established [7, 15].

To enable observation through fluorescence microscopy, lipid-bound fluorescent dyes are added to lipid mixtures with a concentration of $1 \mathrm{~mol} \%$. The fluorescent probes are phospholipids like 1,2-Dihexadecanoyl-sn-glycero-3-phosphoethanolamine (DHPE) or DOPE, on whose head group the individual dye molecule (TR or ATTO 390/488) are bound.

Figure 3.2 shows that the emission spectrum of ATTO 390, in contrast to ATTO 488, only slightly overlaps with that of Texas Red. 
<smiles>CC1=CC(=O)Cc2cc3c(cc21)C(C)CC(C)(C)N3CCCC(=O)O</smiles>

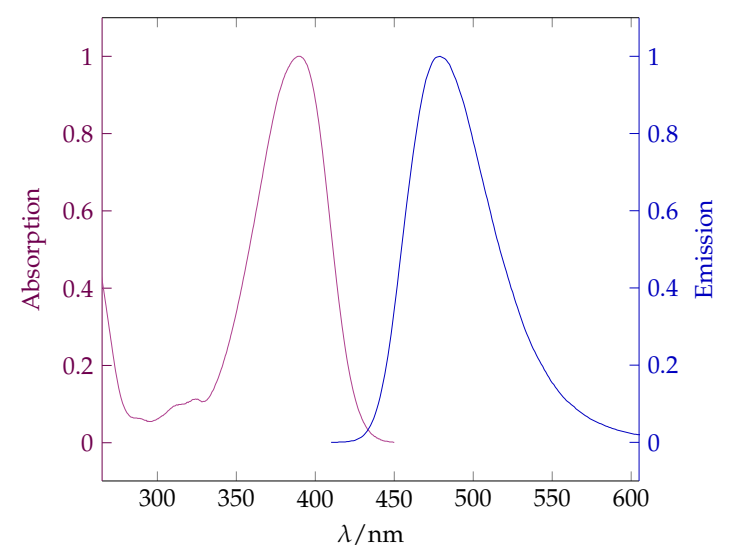

(a) ATTO 390: excitation at $\lambda_{\max }=390 \mathrm{~nm}$, emission at $\lambda_{\max }=479 \mathrm{~nm}$

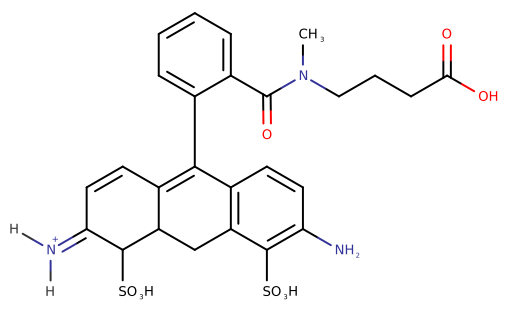

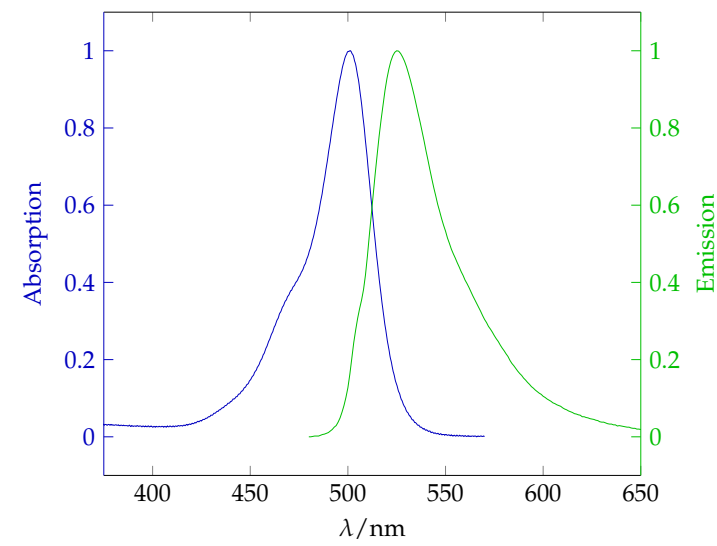

(b) ATTO 488,: excitation at $\lambda_{\max }=501 \mathrm{~nm}$, emission at $\lambda_{\max }=525 \mathrm{~nm}$<smiles></smiles>

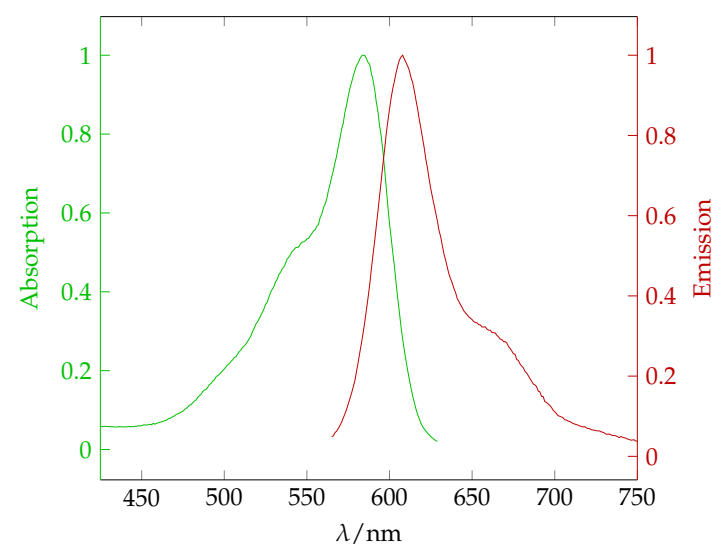

(c) Texas Red ${ }^{\circledR}$, excitation at $\lambda_{\max }=584 \mathrm{~nm}$, emission at $\lambda_{\max }=608 \mathrm{~nm}$

Figure 3.1: Structural formula (left) and absorption or emission spectrum (right) from ATTO390, ATTO488 and Texas Red ${ }^{\circledR}$

\subsection{Materials}

All materials are purchased from commercially available suppliers (Dow Corning, Sigma Aldrich, and Kisker BioTech) and used without further purification. 


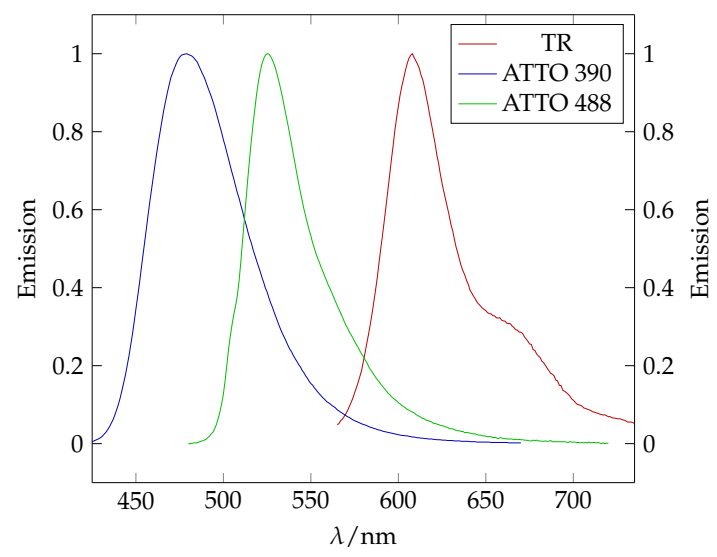

Figure 3.2: Comparison of the emission spectra of the fluorescent dyes used Texas $\operatorname{Red}^{\circledR}$, ATTO 390 and ATTO 488.

\subsubsection{Unilamellar vesicles}

Liposomes, or lipid vesicles, are artificially prepared spherical structures composed of one or a few lipid bilayers formed in vitro in an aqueous medium. This closed system is energetically desirable because it prevents the water from exposing the hydrophobic tails. Liposomes are also differentiated according to the size and scale of their lamellarity. Depending on lamellarity, liposomes can be divided into unilamerllar vesicles and mutilamerllar vesicle. Unilamellar vesicles are a model system that is easy to prepare and, compared to free-standing membranes, a widely applicable model system for mimicking cell membranes' biophysical properties. All vesicles consist of a spherical lipid double layer that includes an aqueous compartment. There are generally three different size ranges of unilamellar lipid vesicles [21]:

- small unilamellar veiscle , $\mathrm{d}=20-50 \mathrm{~nm}$

- large unilamellar veiscle, $\mathrm{d}=100-1000 \mathrm{~nm}$

- giant unilamellar veiscle, $\mathrm{d}=$ up to $150 \mu \mathrm{m}$

Various methods have been developed to prepare liposomes [1, 28]. First, to ensure a homogeneous mixture, a lipid mixture of the desired composition is dissolved in an organic solvent. Then the solvent is evaporated to produce a dry lipid film. By applying an aqueous solution and agitating above the lipids' transition temperature, hydration of the dry lipid layer is achieved, creating multilamellar vesicles (MLVs) of various size distributions. Once a stable MLV suspension form, extrusion or sonication will downsize the vesicles. LUVs of the appropriate size can be produced by extrusion through a polycarbonate filter with given pore size. Sonication will interrupt the suspension to create bilayer fragments and assemble them into SUVs ultimately. 


\subsubsection{Glass substrate}

Supported membranes can be formed upon a variety of solid substrates. These solid supports rigidly confine the membrane to their surface and thereby suppress the membrane's freedom to bend in response to interactions with proteins and other membrane-active matter. For functionalization, and because of optical transparency for visual inspection of bilayer formation, glass substrates were used as solid supports. Optical microscopy experiments and force spectroscopy experiments were conducted on glass-bottom Petri-dishes (Mattek Corporation, Ashland, MA, USA), $\mu$-Slides ( $\mu$ Slides 0.5, Ibidi, Martinsried, Germany), plasma-activated silicon dioxide wafer, and PDMS. For the preparation of a homogenous lipid membrane on glass substrates, the glass substrates were incubated in a 1-2\% Hellmanex III (Hellma Analytics, Müllheim, Germany) solution at room temperature overnight, rinsed multiple times with ultrapure water (MilliQ), before dried under a gentle stream of nitrogen.

\subsubsection{Silica beads}

The silica beads were used as a membrane template to prepare solid-supported bilayer beads, called membrane-coated beads. These uniform, non-porous silica beads of different mean diameters $0.9 \mu \mathrm{m}$ to $15 \mu \mathrm{m}$, were collected from Bangs Laboratories (Fisher, IN, USA). Silica beads were kept at $4{ }^{\circ} \mathrm{C}$ in DI water and used without any further modification as obtained.

\subsubsection{Polydimethylsiloxan}

PDMS is a colorless, transparent, inorganic polymer made of dimethylsilyl groups connected in a chain via oxygen bridges. It is considered non-poisonous as well as being chemically inert. When preparing this as elastic support, the used material was a two-component kit from Dow Corning (West Midland, USA). The kit is sold commercially as Sylgard 184 and consists of a prepolymer and a hardener, which initiates polymerization. If both components are put together, they polymerize into one solid and cross-linked elastomer with defined mechanical properties. The hardening can occur at room temperature or, for a shorter period, under heat in the drying cabinet. After curing, the surface was modified in oxygen plasma to ensure the membrane's adhesion to the PDMS support. The treatment in the oxygen plasma generates free hydroxyl groups on the surface, which increases surface tension. On the one hand, this creates covalent bonds to other substrates, such as glass carriers, glass spheres, or PDMS, and promotes the spreading of vesicles on the surface. [9]. We used this approach to covalently attach $15 \mu \mathrm{m}$ spheres to a transparent PDMS substrates and 
spread a lipid bilayer afterward onto these spheres, creating a stable three-dimensional curved lipid wall for optical tweezer experiments (Fig. 3.4). Or to make non-continuous bilayer patches with defined diameters in $\mu \mathrm{m}$ range on PDMS (Fig. 3.3). The used method produced small bilayer patches of $5 \mu \mathrm{m}$ diameter.

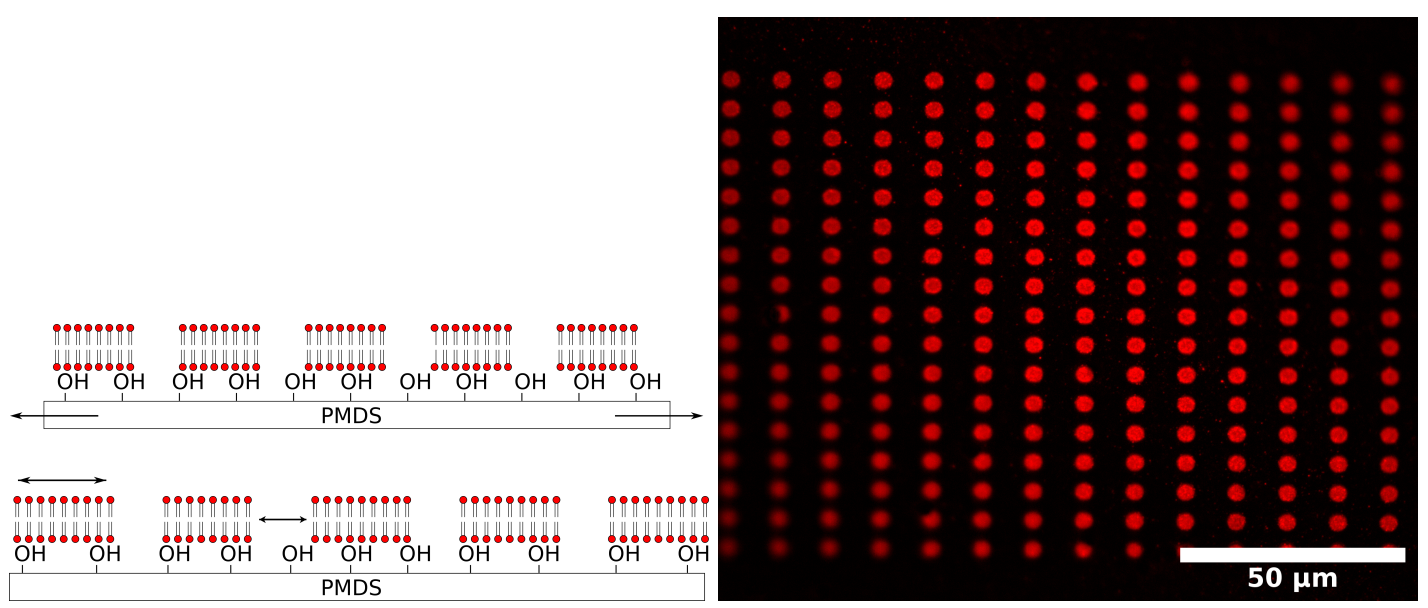

Figure 3.3: Membrane bilayer patches. Illustration of membrane bilayer patches of defined size adhered on oxidized PDMS substrate (left). Fluorescence microscope image of DOPC:DOPS:DOPE:Chol:TR bilayer patches on PDMS (right).
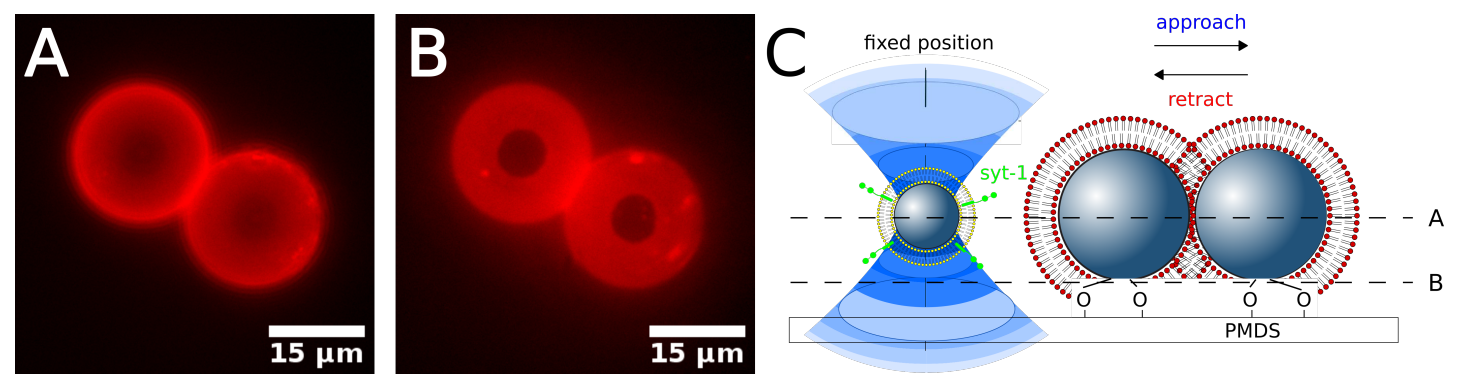

Figure 3.4: Vertical bilayer support for OT-experiments. A-B: fluorescence images of membrane coated beads covalently bond to PDMS from center (A) and bottom (B) vertical focal plane acting as target membrane DOPC:DOPS:DOPE:CHOL:PIP 2 :TexasRed (46:20:20:11:1:2) for an optical tweezer force spectroscopy approach C. C: Illustration of OT experimental approach for force spectroscopy of Syt-1 DOPC:DOPS:DOPE:Chol/OG (62:5:20:11:2) binding against a target membrane.

PDMS was prepared in an Ibidi polymer coverslip bottom dish as described above. A silicon microsieve chip covered it with $5 \mu \mathrm{m}$ pore size in the $\mathrm{SiO}_{2}$ porous area (rectangular, Aquamarijn, Zutphen, Netherlands). This facilitated oxidation of defined $5 \mu \mathrm{m}$ patches of PDMS by oxygen plasma (30s at 20 mbar and 20\% power, Diener Zepto plasma cleaner, Diener Electronic GmbH, Ebhausen, Germany). Subsequently, the microsieve chip was removed, and vesicle spreading was performed as described in the following. 


\subsection{Artificial bilayers and protein reconsitution}

Small unilamellar vesicles (SUVs) are used to produce bilayers by spreading vesicles composed of the desired lipid mixture on glass, silicon dioxide $\left(\mathrm{SiO}_{2}\right)$ or $\mathrm{O}_{2}$ oxidized PDMS substrates above the highest main phase transition temperature of the used lipids. The attachment and fusion of the vesicles on the chosen substrate result in a homogenous coverage of the surface with a lipid bilayer.

\subsubsection{Preparation of lipid films}

The lipid mixtures used for the preparation of proteoliposomes consisted of the phospholipids DOPC, POPE, POPS, PI $(4,5) \mathrm{P}_{2}$, and cholesterol. The ratios of the named phospholipids and cholesterol were varied depending on the focus of the experiment. For the preparation of lipid mixtures with defined composition, the individual lipids' stock solutions were combined. The stock solutions were prepared by dissolving the respective lipid in chloroform with the exception of $\mathrm{PI}(4,5) \mathrm{P}_{2}$, which was dissolved in methanol/chloroform/water (2:1:0.8). After combining each lipid stock solution's defined volumes to achieve the desired molar ratios, the solvents were evaporated by purging with nitrogen gas and heating to $30^{\circ} \mathrm{C}$. To remove any residual solvent, the lipid films were further dried under vacuum for at least $3 \mathrm{~h}$ at $30^{\circ} \mathrm{C}$. All lipid films were prepared with a total mass of $0.25 \mathrm{mg}$. If not used immediately, the obtained lipid films were sealed with Parafilm and stored at $4{ }^{\circ} \mathrm{C}$, protected from light.

\subsection{Reconstitution of SNARE proteins into liposomes}

Freshly degassed SNARE buffer (20 mM HEPES, 100 mM KCl, 1 mM DTT, 0.1 mM EGTA, pH 7.4) was used for all preparation steps. DTT was always added freshly after 30 min of degassing under vacuum and the buffer was stored protected from light.

\subsubsection{Preparation of small proteoliposomes}

The reconstitution of SNARE-proteins into small unilamellar vesicles (SUVs) was performed in accordance with a protocol developed by Hernandes [102]. That protocol is based on a detergent mediated protein reconstitution forming mixed micelles composed of lipids, detergent and protein in the first step. The subsequent formation of SUVs doped with the applied transmembrane protein out of the mixed micelles suspension was done by rapid dilution and multiple gel filtration steps to efficiently remove the detergent. The following reconstitution protocol is the general procedure used for the 
insertion of syb 2 and $\Delta N 49$ into SUVs. For the co-reconstitution of syb 2 and syt- 1 into liposomes the protocol had to be changed due to a higher sensitivity for denaturation of syt-1.

Solubilization of the lipid film in SNARE-buffer $(50 \mu \mathrm{l})$ containing the detergent n-octyl$\beta$-D-thioglucopyranoside $\mathrm{n}$-OG, $\mathrm{c}(\mathrm{n}$-OG $)=75 \mathrm{mM})$ started the reconstitution procedure. A final protein to lipid ratio of $\mathrm{p} / 1=1: 500$ was applied to this mixture by changing the protein solution. The resulting mixed micelles suspension was incubated at $0{ }^{\circ} \mathrm{C}$ for $45 \mathrm{~min}$. The detergent was removed in SNARE-buffer through rapid dilution and size exclusion chromatography (Illustra NAP-10 G25 column, GE Healthcare, Little Chalfont, UK). This extracts salts and residual detergent yielding proteo-SUVs in buffer that served as the source for the formation of solid supported membranes on planar and colloidal templates.

\subsubsection{Reconstitution of syt-1 into liposomes}

The next step was to reconstitute syt- 1 into artificial liposomes to analyze its $\mathrm{Ca}^{2+}$ -independent binding affinity for solid supported membranes (SSMs) doped with the polyanionic phospholipid $\mathrm{PI}(4,5) \mathrm{P}_{2}$. To this point, syt-1 was introduced in a protein to lipid ratio of $\mathrm{p} / 1=1: 1000$ into small unilamellar vesicles (SUVs) using the comicellization protocol according to Dr. Raphael Hubrich [10] or provided by Dr. Angel Perez Lara [18, 27].

\subsubsection{Co-reconstitution of syt-1 and syb 2 into liposomes}

The so-called direct insertion method was applied for the co-reconstitution of syb-2 and syt- 1 into small unilamellar vesicles. In this process, with the detergent n-OG, preformed SUVs were destabilized, followed by adding the relevant proteins from frozen aliquots. Size exclusion chromatography and dialysis were performed to remove the detergent subsequently.

The co-reconstitution protocol [10] started with the preparation of SUVs. For this purpose, a lipid film of $0.5 \mathrm{mg}$ was allowed to swell in SNARE buffer (500 $\mu \mathrm{l})$ for $30 \mathrm{~min}$ at room temperature. During this incubation period, the mixture was vortexed three times for $30 \mathrm{~s}$ in a $5 \mathrm{~min}$ time interval, yielding an opaque suspension of multilamellar vesicles, and transferred to a $1.5 \mathrm{ml}$ microcentrifuge tube. SUVs were obtained by sonication for 30 minutes in a Bandelin Sonopuls HD2070 sonifier equipped with a BR30 beaker resonator and an EH3 reaction cup holder (Bandelin Electronic, Berlin, Germany), at $65 \%$ power and a $4 \times 10 \%$ pulsed cycle. The resulting SUVs were destabilized 
by addition of $\mathrm{n}-\mathrm{OG}(\mathrm{c}(\mathrm{n}-\mathrm{OG})=26 \mathrm{mM})$, followed by incubation for $10 \mathrm{~min}$ at room temperature. Subsequently, protein solutions of syb-2 and syt- 1 were added, adjusting a protein/lipid-ratio of 1:500 and 1:1000, respectively. This mixture was further incubated at room temperature for $30 \mathrm{~min}$, followed by size exclusion chromatography in MilliQ/SNARE buffer (9:1) using a G-25 pre-packed column (Illustra NAP-25 G-25, GE Healthcare, Little Chalfont, UK). After size exclusion, the proteo-SUVs containing fraction $(850 \mathrm{ml})$ were used to prepare membrane coated glass spheres and the subsequent fusion experiments.

\subsubsection{Membrane-coated glass spheres}

Membrane-coated glass spheres were prepared according to the method described by Bao et al.. [2] A suspension of glass spheres (10 $\mu \mathrm{l}, 10 \mathrm{wt} \%)$ with $250 \mu \mathrm{l}$ spreading buffer (10 mM HEPES, $300 \mathrm{mM} \mathrm{NaCl}$, pH 7.4) is incubated with $250 \mu \mathrm{l}$ SUV solution for $30 \mathrm{~min}$ and swirled continuously on a pulse vortexer to ensure that the vesicles spread evenly on the spherical surface. Excess SUVs in the supernatant were removed by repeated centrifugation ( $5 \mathrm{~s}, 6600 \mathrm{rpm}, \mathrm{LMS}$ MCF-2360 mini-centrifuge, Heidelberg, Germany) and rinsed with $6 \times 500 \mu l$ buffer separated from the sphere suspension.

Hence proteins on solid supported membranes can be prone to physical damage after insertion and co-reconstitution; removing excessive proteo-SUVs was performed by sedimentation of beads through a $5 \mathrm{ml}$ pipette tip as shown in Figure 3.5 .
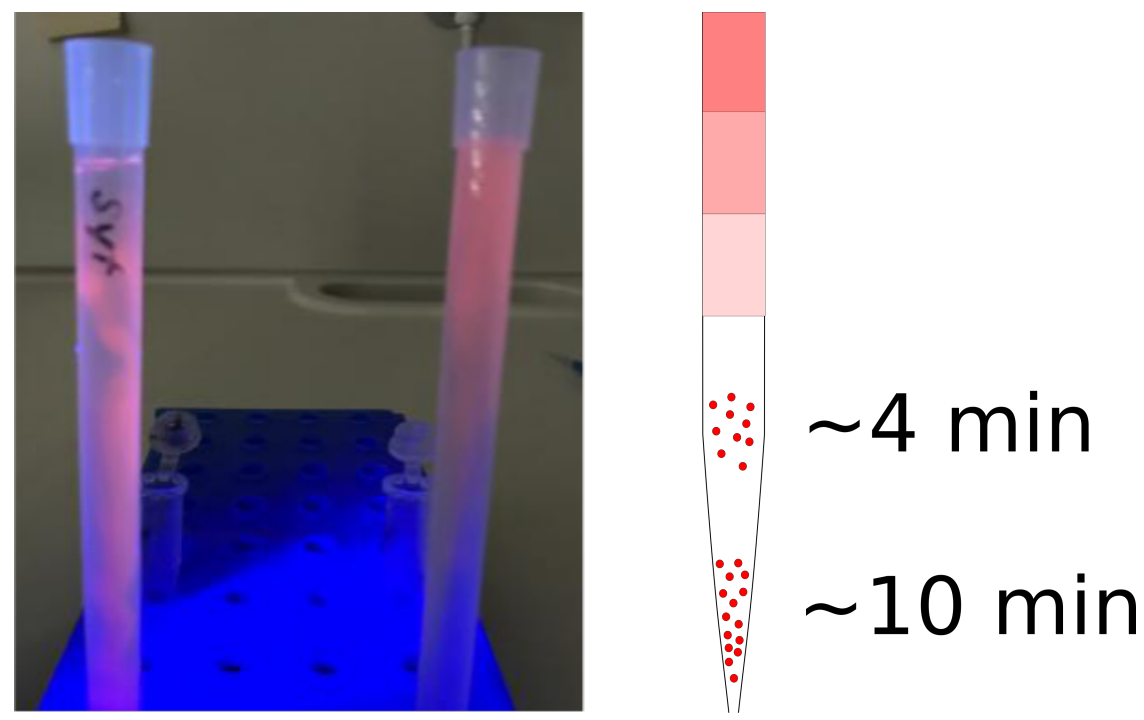

Figure 3.5: Fluorescence excitation of TexasRed labeled membrane-coated glass spheres with a particle size of $4.7 \mu \mathrm{m}$ sedimenting through a pipette column.

Successful preparation of membrane-coated glass spheres was verified using a fluorescent microscopy (Fig. 3.6) and FRAP analysis. The membrane-coated spheres produced 
in this way were stored at $4{ }^{\circ} \mathrm{C}$ for further use under exclusion of light or used imminently.

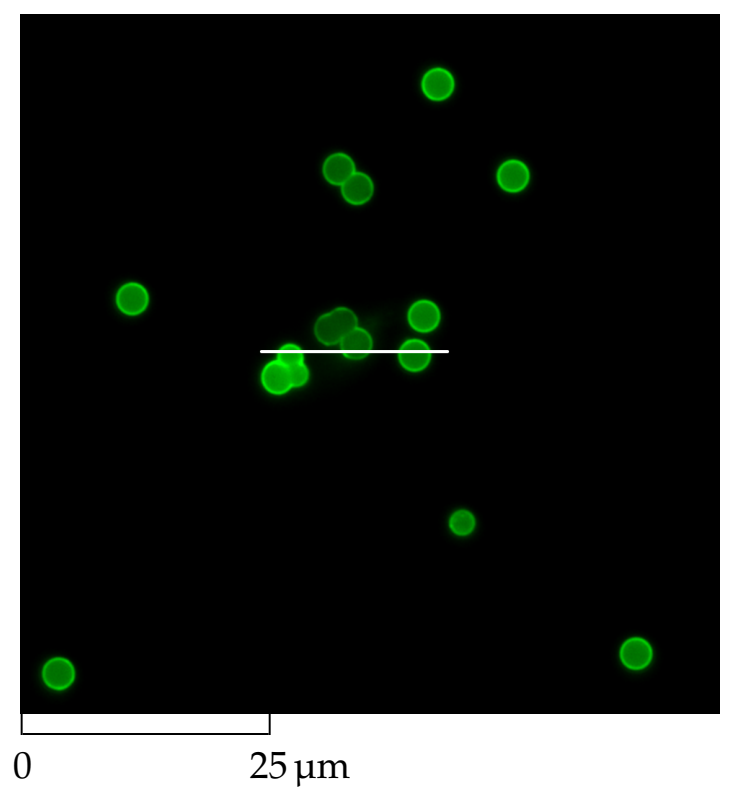

(a) fluorescence microscope image of various membrane-coated glass spheres

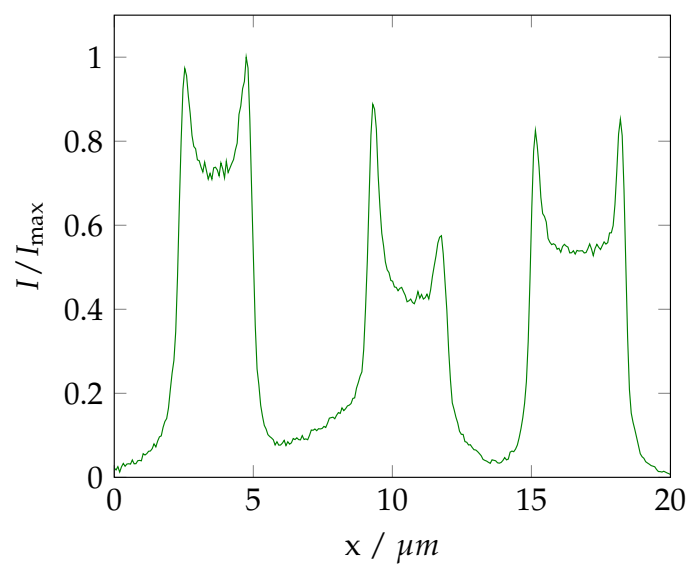

(b) line profile

Figure 3.6: Fluorescence microscope image of ATTO 488 DOPE labeled membranecoated glass spheres (left) with a particle size of $4.7 \mu \mathrm{m}$, with a corresponding line profile (right).

\subsection{Analytical fluorescence microscopy}

Two fluorescence microscopes were used in this work. The upright microscope $B X$ 51 (Olympus, Tokyo, Japen) with a CCD camera (DP 71, Olympus, Tokio, Japan) was used for the fluorescence microscopic examination of the membrane spreading on oxidized PDMS surfaces, and the control of all preparative steps was performed using DP 71, Olympus, Tokyo, Japan) using a LUM-Plan-FLN water immersion objective (Olympus, Tokyo, Japan) with 20, 40 or 100 times magnification and a mercury vapor lamp (X-Cite 120Q, Lumen Dynamics Group, Ontario, Canada). An upright confocal laser scanning microscope was used for long-term fluorescence microscopic recordings, the photobleaching process, and the fusion experiments. The setup used consisted of a AXIO LSM 710 (Zeiss, Jena, Germany) and a W-Plan Apochromat water immersion objective (Zeiss, Jena, Germany) with 20-, 40 or 63 times magnification. An argon laser (Lasos Lasertechnik, Jena, Germany) was used as the light source. To determine the intensity values, either the software ZEN (Zeiss, Jena, Germany) or the platformindependent image processing and image processing program ImageJ. Written by Wayne Rasband in Java used. 


\subsubsection{Incident light fluorescence microscopy}

Incident light fluorescence microscopy is a fundamental investigation technique in which the spontaneous emission of light occurs during the transition from the vibronic ground state of an electronically excited singlet state $S_{1}$ back to the ground state of low energy $\mathrm{S}_{0}$ (see Fig. 3.7 a). A mercury-vapor lamp is used as the light source. The light hits an excitation filter that only allows the light of a specific wavelength to pass through. This light is focused on the sample via a dichroic mirror and focused through the objective. The irradiated light excites the fluorophore to fluorescence. The emitted light hits an emission filter through the lens and the dichroic mirror, ensuring that only emitted light of a particular wavelength is allowed. The light is captured by a digital camera with a CCD sensor. Figure $3.7 \mathrm{~b}$ illustrates the schematic structure of such a fluorescence microscope.

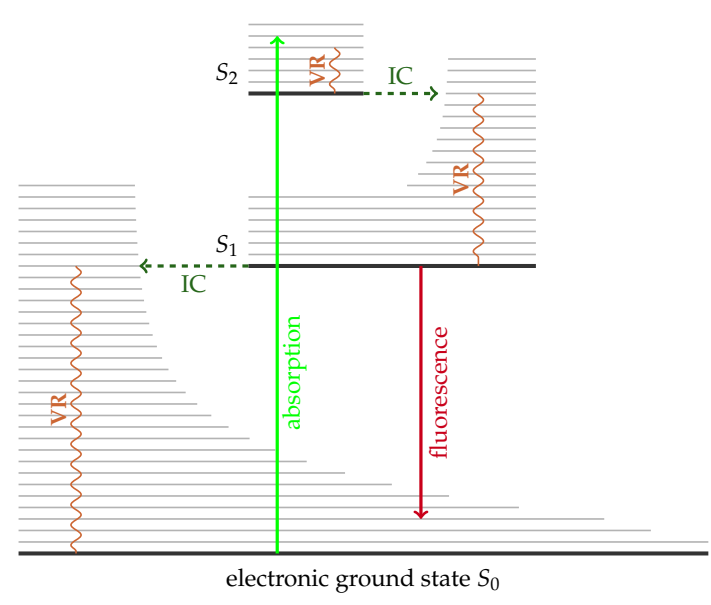

(a) JABLONSKI-diagram

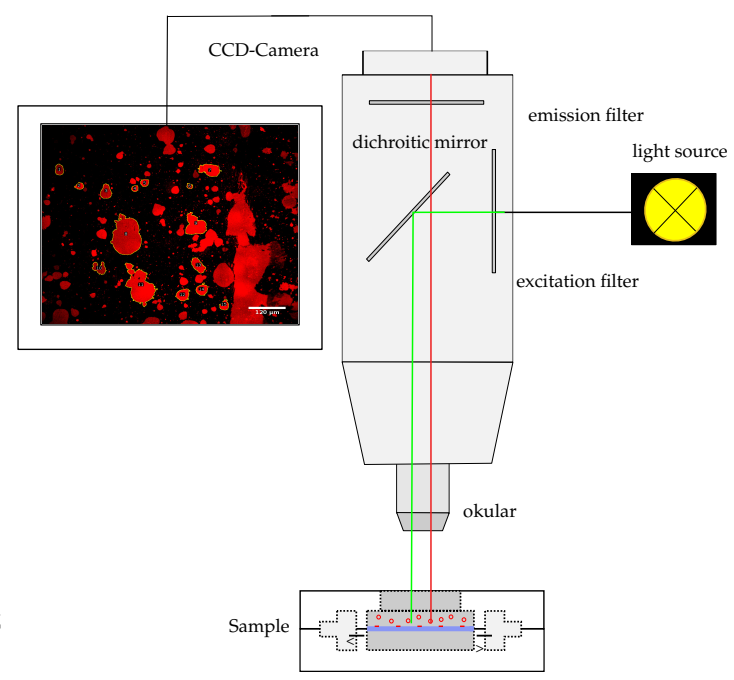

(b) Beam path

Figure 3.7: Jablonski diagram of possible transitions between the electronic states S0, S1 and $\mathrm{S} 2$ as well as the schematic structure and beam path of a reflected light fluorescence microscope.

\subsubsection{Confocal fluorescence microscopy}

In confocal microscopy, a special light microscope is used, which only keeps one point of the sample in focus at any one time. A point-shaped light source is focused on the sample via a lens or an objective, the spatial extent of the focal point being determined by the wavelength of the light source and the objective lens used. The focused image point is then aligned through the same lens to a pinhole in front of the detector. The pinhole's size is chosen so that only light from the beam path's sharply imaged area is allowed through, and light from other planes is blocked. The point in the middle 
of the pinhole and the illumination point in the specimen are confocal (lat. con, focal: having the same focus) to one another. This significantly increases the microscopic image's contrast since only light from the focal plane reaches the detector, and hardly any backscatter is measured.

\subsubsection{Fluorescence recovery after photobleaching}

Fluorescence recovery after photobleaching FRAP was used to investigate qualitative information about the dynamic and structural properties of the adhered lipid bilayers and their lateral mobility to ensure bilayer deposition. The quantitative evaluation was carried out by determining the half-life of the fluorescence return and by calculating the diffusion coefficient [24, 34].

A high-energy laser pulse is applied to the dye in a defined region of the sample (region of interest, ROI) irreversibly decomposed, so that a fading bleaching of the dye can be observed in the ROI. Subsequently, the exchange of bleached molecules with lipids from the environment, i.e. the temporal recovery of the fluorescence intensity, is observed through diffusion (Fig. 3.8).

In the case of the approximation formula, the half-life was read off graphically from the plot of intensity against time. The Gaussian radius was calculated from the area of the bleaching spot. The diffusion coefficient could be calculated from the half-life $\tau_{1 / 2}$ of the exponential fluorescence return.

Assuming a Gaussian profile for the bleaching point and a round bleaching area $\left(\omega^{2}\right)$, the diffusion coefficient $D$ was calculated by simple-exponential adjustment of certain half-lives according to the following first approximation:

$$
\begin{aligned}
f_{(t)} & =A \cdot(1-\exp (\tau \cdot t)) \\
\tau_{1 / 2} & =\frac{\ln (0.5)}{-\tau} \\
D & =\frac{\omega^{2}}{4 \cdot \tau_{1 / 2}}
\end{aligned}
$$

In order to compare the FRAP experiments, as described by Phair et al. [19, 29], The measured FRAP data was normalized twice by subtracting the background signal 


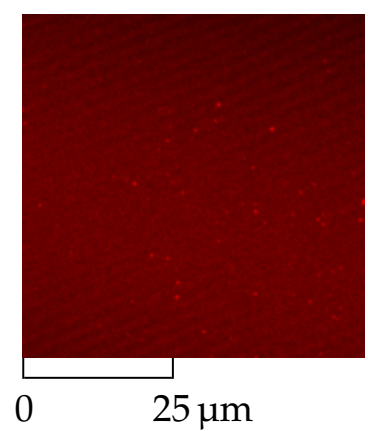

(a)

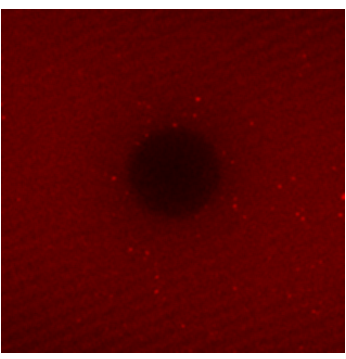

(b)

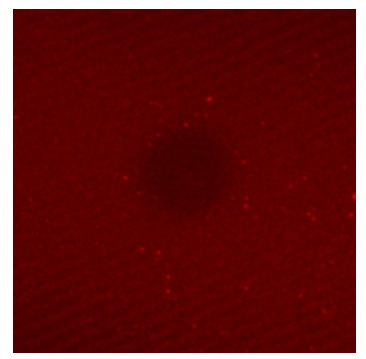

(c)

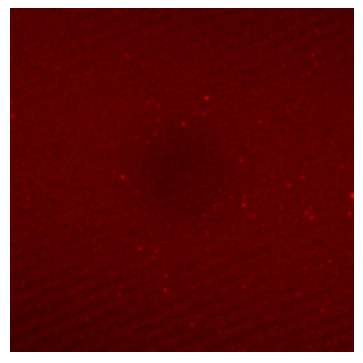

(d)
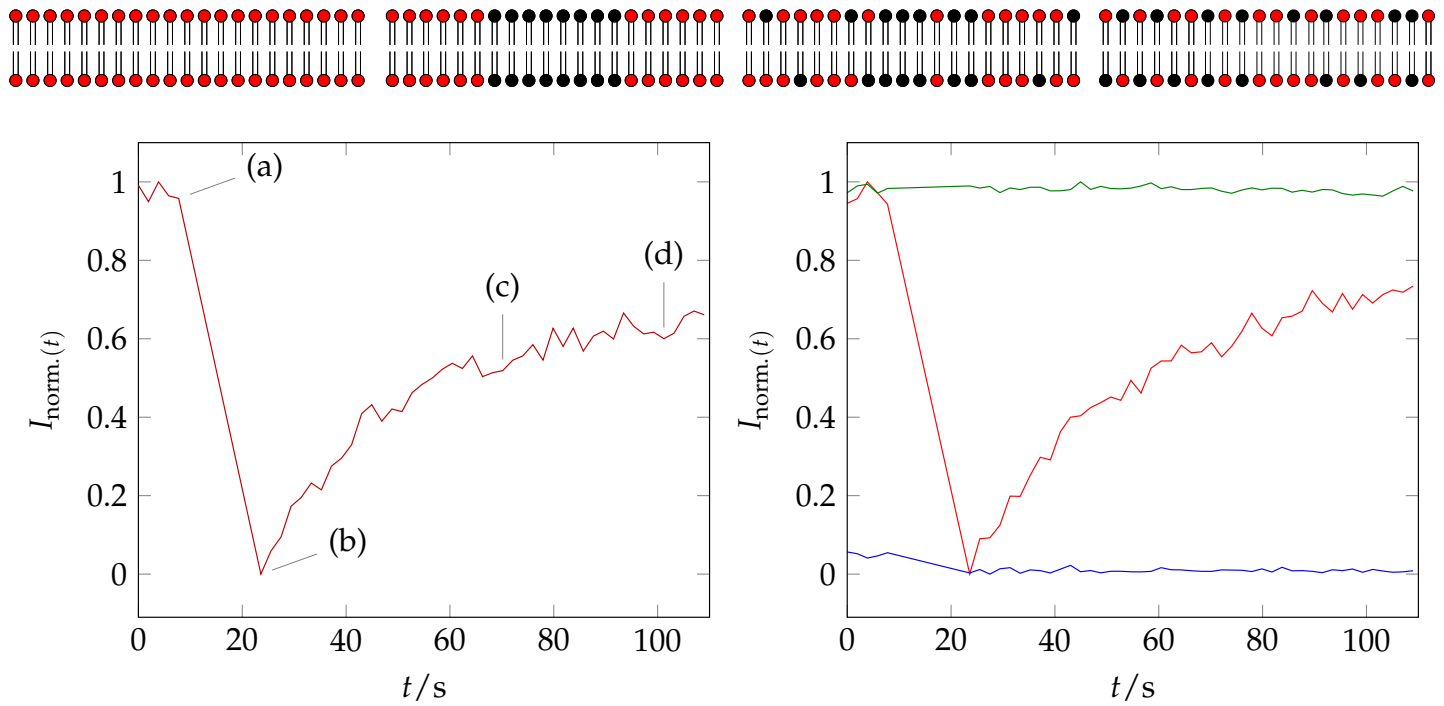

Figure 3.8: Exemplary representation of a FRAP experiment: (a) fluorescence intensity before bleaching; (b) quenching the fluorescence in the ROI by bleaching; (c) regeneration of the fluorescence intensity in the ROI by diffusion; (d) compared to fully regenerated fluorescence with reduced intensity (a). - FRAP, - reference, - background.

with normalization to the signal intensity at the point in time before the sample was bleached with simultaneous correction of one reference ROIs so that the FRAP intensity remains distinguishable from the general bleaching of the entire sample during the measurement [29].

$$
I_{\text {norm. }}(t)=\frac{I_{\text {ref,pre }}}{I_{\text {ref }}(t)-I_{\text {back }}(t)} \cdot \frac{I_{\text {frap }}(t)-I_{\text {back }}(t)}{I_{\text {frap pre }}}
$$

With no background ROI, a simple normalization of the FRAP data was performed:

$$
I_{\text {norm. }}(t)=\frac{I_{\text {frap }}(t)-I_{\text {back }}(t)}{I_{\text {frap,pre }}}
$$


We routinely carried out FRAP measurements to ensure lateral bilayer mobility and successful membrane formation after vesicle spreading. Figure 3.9 shows exemplary fluorescence images of target SSMs prepared on a glass surface to study the influence of PIP $(4,5)_{2}$ on the SNARE mediated membrane fusion.

\subsubsection{Preparation of solid support membranes for influence of $\mathrm{PI}(4,5) \mathrm{P}_{2}$ on SNARE mediated membrane fusion}

The preparation of SSM started with the addition of SUVs composed of DOPC:POPE: POPS:Chol:PI(4,5) $\mathrm{P}_{2}\left(45: 20: 10: 20: 5,0.2 \mathrm{mg} \mathrm{ml}^{-1}\right)$ in citrate buffer NaC20 (20 mM Nacitrate, $50 \mathrm{mM} \mathrm{KCl}, 0.1 \mathrm{mM}$ EDTA, $0.1 \mathrm{mM} \mathrm{NaN}_{3}$, $\mathrm{pH} 4.8$ ) to a glass, silicon dioxide $\left(\mathrm{SiO}_{2}\right)$ or $\mathrm{O}_{2}$ oxidized PDMS substrates and was finished after $30 \mathrm{~min}$. The citrate buffer with a $\mathrm{pH}$ of 4.8 was needed to facilitate the spreading process in case of $\mathrm{PI}(4,5) \mathrm{P}_{2}$ concentrations of $5 \mathrm{~mol} \%$ to reduce the negative surface charge of the SUVs by protonating the $\mathrm{PI}(4,5) \mathrm{P}_{2}$ headgroup. For this reason a buffer exchange was carried out after the completed formation of the SSM by rinsing the measuring chamber in an open system with SNARE buffer with at least $10 \mathrm{ml}$.

Planar bilayer formation and coverage of the substrate was controlled by confocal laser fluorescence microscopy and fluorescence recovery after photobleaching (FRAP) measurements (Fig 3.9). Only samples with a surface coverage over $95 \%$ and mobile bilayer formation were used for subsequent experiments.

\subsection{Preparation of the colloidal probe cantilever}

Colloidal probe cantilevers, as shown in Figure 3.10, were prepared by attaching a borosilicate glass microsphere with a diameter of $15 \mu \mathrm{m}$ (Duke borosilicate glass 9015, Duke Scientific Corporation, Palo Alto, CA, USA) to a tipless MLCT-O10 cantilever (Bruker AFM Probes, Camarillo, CA, USA). The attachment was achieved by using melted epoxy resin at a temperature of above $110^{\circ} \mathrm{C}$ (Epikote 1004, Brenntag GmbH, Mühlheim, Germany 24) as a glue. An upright light microscope (Olympus BX 51, Hamburg, Germany) with a 20x magnification equipped with a nano manipulator (MM3A-LS, Kleindiek Nanotechnik GmbH, Reutlingen, Germany) was used for positioning.

Before bilayer preparation on the colloidal probe, the cantilevers were cleaned in argon plasma for at least $30 \mathrm{~s}$. Afterward, the colloidal probe cantilever was mounted to the AFM-head and incubated with a (proteo)-liposome solution ( $80 \mu \mathrm{l}$ to $100 \mu \mathrm{l}, 3 \mathrm{mM}$ in HP150 buffer) in a free-hanging droplet at least $25 \mathrm{~min}$ at room temperature. Excess 


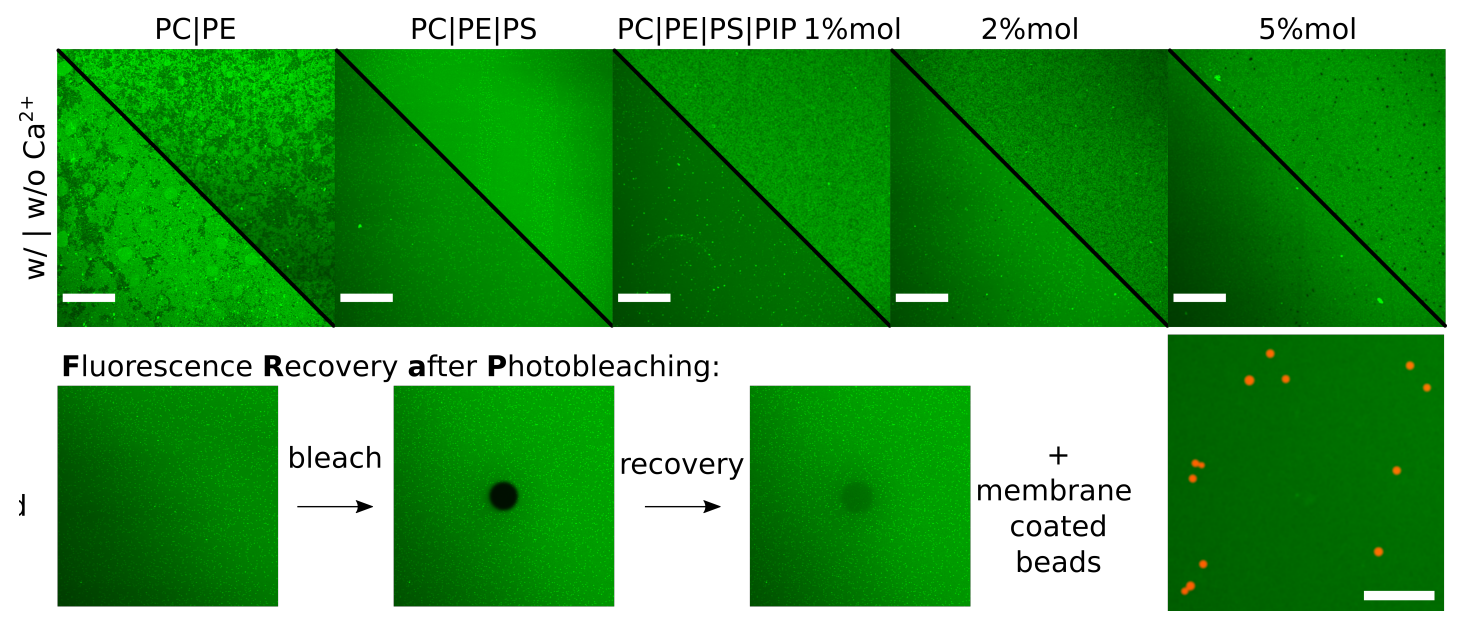

Figure 3.9: (A) Exemplary fluorescence images of target SSMs prepared on a glass surface to study the influence of $\operatorname{PIP}(4,5)_{2}$ on the SNARE mediated membrane fusion. As target membrane DOPC:POPE:POPS:Chol:PIP $(4,5)_{2}$ :Atto488-DPPE varying with PS and different PIP $(4,5)_{2}$ concentraions $n$ (50:29:0:20:0:1, 50:19:10:20:0:1, 50- $n: 19: 10: 20: n: 1$ $n=1 \%, 2 \%, 5 \%$ ) are used. (B) Exemplary FRAP measurements on a glass surface covered with a supported lipid bilayer containing Atto488-DPPE as fluorescent dye. The fluorescent images shown in a time series before and after bleaching. A $561 \mathrm{~nm}$ LASER bleached the dye lipid in the SSM in a defined region of interest (ROI). Recovery occurs due to the diffusion of the fluorescently labeled lipids from the surrounding of the bleached area. After time the fluorescence homogenity recovers and ensures mobility of deployed bilayers. (C) Addition of $1 \mu \mathrm{m}$ glass microspheres covered with lipid membrane consisting of DOPC:POPE:Chol:TR-PE (50:29:20:1) with or without reconstituted $\mathrm{f}-\mathrm{Syt}-1$ under containing and non-containing $\mathrm{CaCl}_{2}$-buffer conditions were performed. Scale bar $25 \mu \mathrm{m}$

vesicle solution was removed by rinsing with HP150 buffer $(1.5 \mathrm{ml})$.

Force-distance measurements were performed with an MFP3D (Asylum Research, Santa Barbara, CA, USA). The cantilevers spring constants were calibrated using the thermal noise method and obtained to be in a range of 6-12 $\mathrm{pN} / \mathrm{nm}$.

All force-distance cycles were operated with a forward velocity of $500 \mathrm{~nm} / \mathrm{s}$, varying retraction velocity $(100 \mathrm{~nm} / \mathrm{s}, 500 \mathrm{~nm} / \mathrm{s}, 1000 \mathrm{~nm} / \mathrm{s}, 2500 \mathrm{~nm} / \mathrm{s}$, and $5000 \mathrm{~nm} / \mathrm{s})$ and contact time ( $3 \mathrm{~s}$ and $10 \mathrm{~s}$ ) at a loading force of $200 \mathrm{pN}$. The measurements were performed in HP150 buffer or in HP150-Ca buffer (20 mM HEPES, $150 \mathrm{mM} \mathrm{KCl}, 1 \mathrm{mM}$ $\left.\mathrm{CaCl}_{2}, \mathrm{pH} 7.4\right)$, respectively.

For each set of parameters, at least 20x20 force-distance cycles over an area of 20x20 $\mu^{2}$ were performed. 


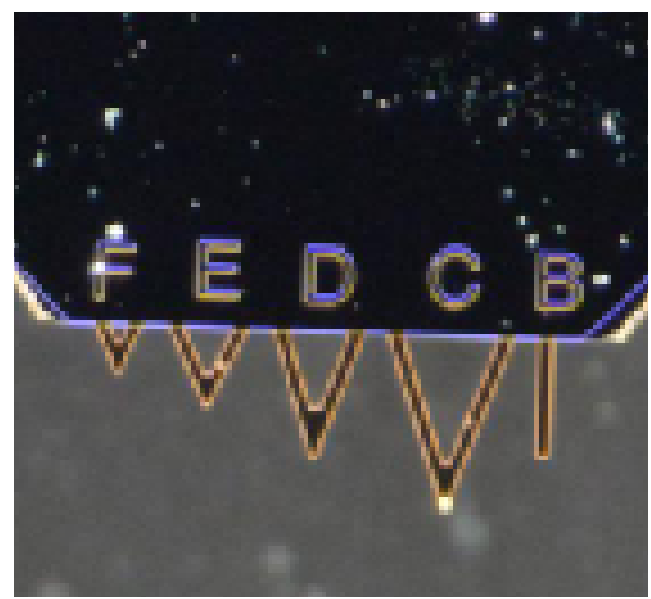

(a) cantilever chip

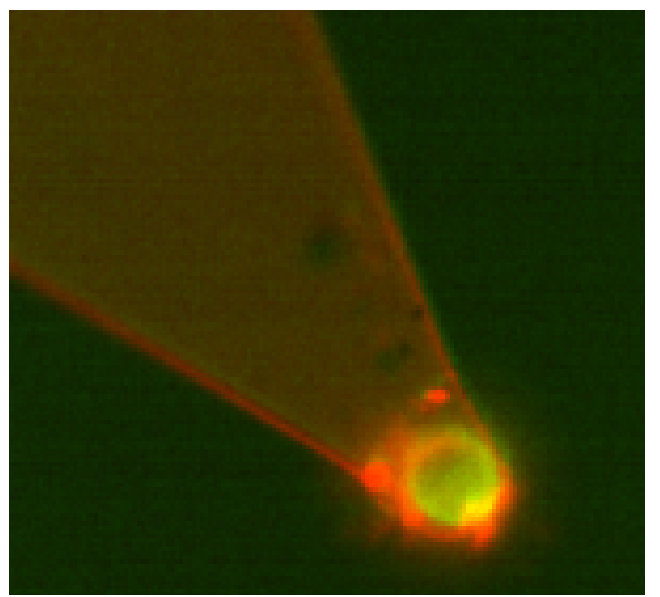

(b) colloidal probe enveloped with a lipid membrane

Figure 3.10: Images of a borosilicate glass microsphere with a diameter of $15 \mu \mathrm{m}$ glued to a tip-less cantilever: (a) Incident-light micrograph with 10x magnification of a cantilever chip (b) Fluroescence micrograph with 60x magnification of an enveloped SSLM as colloidal probe.

\subsection{Cell culture of $\mathrm{C} 2 \mathrm{C} 12$ cell line}

Myocytes are long tubular cells that arise from myoblasts and make up muscle fibers in human beings [33]. C2C12 is an immortalized mouse myoblast cell line. The C2C12 cell line is a subclone of myoblasts that were originally obtained by Yaffe and Saxel at the Weizmann Institute of Science in 1977 [35]. C2C12 cells demonstrate rapid development and maturation into functional skeletal muscle cells or cardiac muscle cells, having the ability to contract and generate force [23]. The rate of muscle formation from C2C12 cells can be controlled by the introduction of loss-of-functions mutations vital for the fusion of myoblasts and myogenesis [5]. These cells provide in vitro models to study differentiation of myoblasts and osteoblasts and mechanistic biochemical pathways [30] ${ }^{1}$.

Cells were purchased from American Type Culture Collection and propagated in DMEM (Gibco) containing 10\% heat-inactivated bovine growth serum (BGS) and supplemented with antibiotics. $\mathrm{C} 2 \mathrm{C} 12$ cells were differentiated by switching to media containing $2 \%$ heat-inactivated horse serum (HS) and antibiotics (Differentiation Medium, DM).

For experiments, cells were seeded in tissue culture treated petri dishes (ibidi $\mu \mathrm{m}$-Dish, low, sometimes with grid (Grid-500), Martinsried, Germany) with $2 \mathrm{ml}$ medium. In case

\footnotetext{
${ }^{1}$ The cell lines used in this work were thankfully cultivated by Angela Rübeling and Dr. Bastian Brückner (University of Göttingen, Germany).
} 


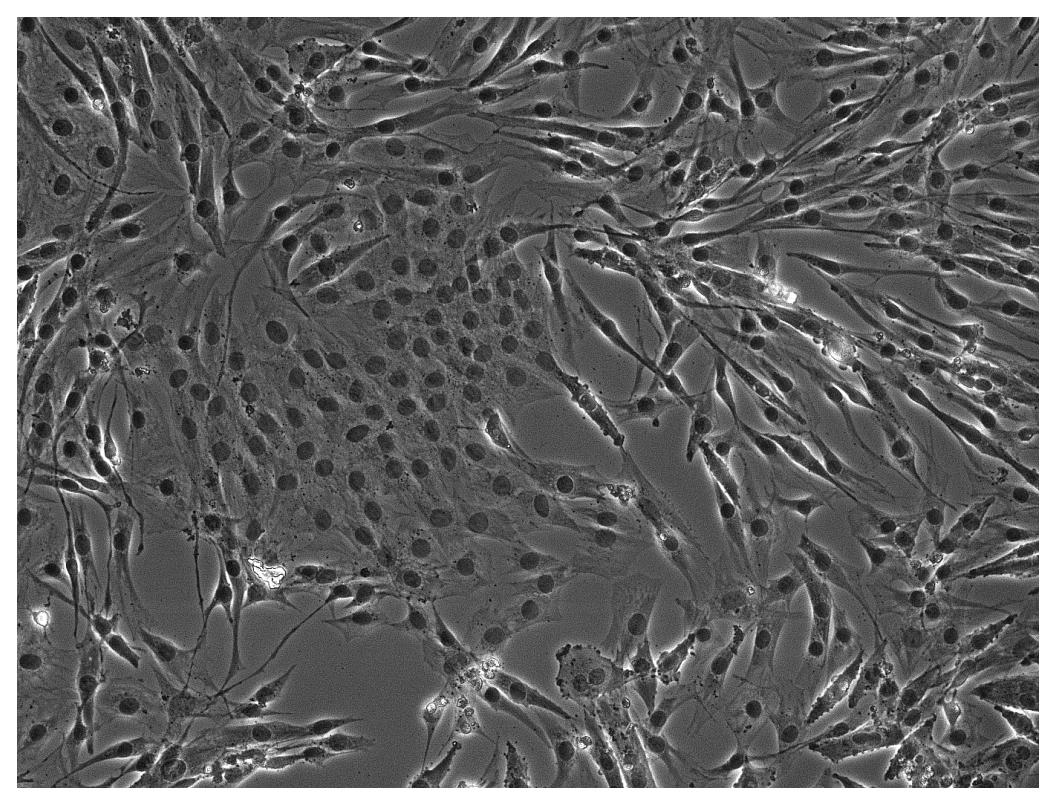

(a) prior differentiation

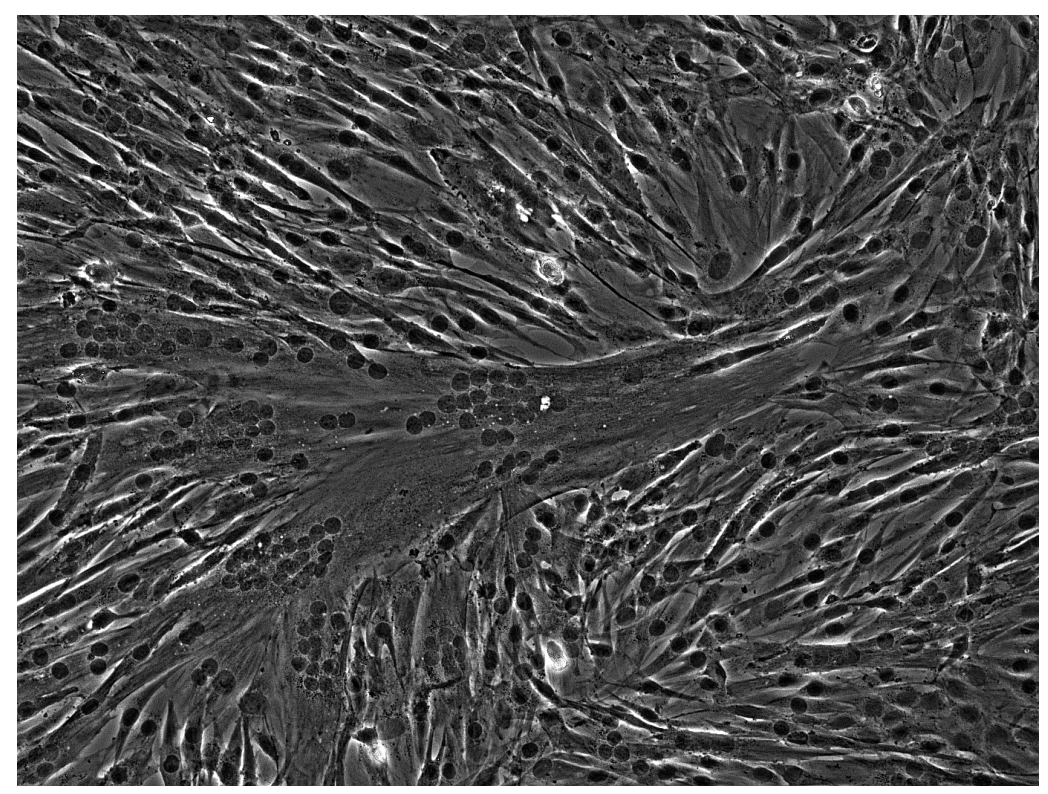

(b) 24 hours after change to DM

Figure 3.11: Phase contrast images of $\mathrm{C} 2 \mathrm{C} 12$ cells at different stages of differentiation $24 \mathrm{~h}$ before and after change of medium.

of no $\mathrm{CO}_{2}$ supply during the experiment, $15 \mathrm{mM}$ HEPES (Biochrom, Berlin, Germany) was added to the culture medium for differentiated and undifferentiated cells. The heating setup (petri dish heater, JPK Instruments, Berlin, Germany) was set to $37^{\circ} \mathrm{C}$. Medium was changed every 2 hours to compensate the slow $\mathrm{pH}$ change observed also in the HEPES buffered medium.

For synchronized fusion, Lysophosphatidylcholine (LPC) was applied 2-3 hours after co-plating cells [12, 17, 26]. The cells were incubated in the LPC-containing complete 
medium for 16 hours. Fusion was scored $30 \mathrm{~min}$ after LPC removal and thus 68 hours after placing the cells into differentiation medium. 


\section{Reference}

[1] Abolfazl Akbarzadeh, Rogaie Rezaei-Sadabady, Soodabeh Davaran, Sang Woo Joo, Nosratollah Zarghami, Younes Hanifehpour, Mohammad Samiei, Mohammad Kouhi, and Kazem Nejati-Koshki. Liposome: Classification, preparation, and applications. Nanoscale Research Letters, 2013. 8(1): 1. doi:10.1186/1556-276X-8-102.

[2] Chunxiao Bao, Gesa Pähler, Burkhard Geil, and Andreas Janshoff. Optical fusion assay based on membrane-coated spheres in a 2D assembly. Journal of the American Chemical Society, 2013. 135(3): 12176-12179.

[3] G Basanez, FM Goni, and A Alonso. Effect of single chain lipids on phospholipase C-promoted vesicle fusion. A test for the stalk hypothesis of membrane fusion. Biochemistry, 1998. 37(11): 3901-3908.

[4] F Benfenati, P Greengard, J Brunner, and M Bähler. Electrostatic and hydrophobic interactions of synapsin I and synapsin I fragments with phospholipid bilayers. The Journal of cell biology, 1989. 108(5): 1851-62.

[5] Barbara A. Bour, Malabika Chakravarti, Joshua M. West, and Susan M. Abmayr. Drosophila SNS, a member of the immunoglobulin superfamily that is essential for myoblast fusion. Genes and Development, 2000. 14(12): 1498-1511. doi:10.1101/ gad.14.12.1498.

[6] Leonid V. Chernomordik and Michael M. Kozlov. Protein-Lipid interplay in fusion and fission of biological membranes. Annual Review of Biochemistry, 2003. 72(1): 175-207.

[7] Hoekstra D. Fluorescence assays to monitor membrane fusion: potential application in biliary lipid secretion and vesicle interactions. Hepatology (Baltimore, $M d$ ), 1990. 12(3 Pt 2): 61S-66S.

[8] Steffen Frey and Dirk Görlich. A new set of highly efficient, tag-cleaving proteases for purifying recombinant proteins. Journal of Chromatography A, 2014. 1337: 95-105. doi:10.1016/j.chroma.2014.02.029.

[9] Jennifer S. Hovis and Steven G. Boxer. Patterning and composition arrays of supported lipid bilayers by microcontact printing. Langmuir, 2001. 17(11): 34003405.

[10] Raphael Hubrich. Pore-spanning membranes - a versatile tool to analyze SNARE- 
mediated single vesicle fusion. Dissertations at Georg-August-Universität Göttingen eDiss, 2018: .

[11] Peter Jönsson, Magnus P Jonsson, Jonas O Tegenfeldt, and Fredrik Höök. A method improving the accuracy of fluorescence recovery after photobleaching analysis. Biophysical journal, 2008. 95(11): 5334-5348.

[12] Manuel Jose Prieto, Ritva Tikkanen, Joseph Szule, Rainer A Böckmann, Jing Han, and Kristyna Pluhackova. The Multifaceted Role of SNARE Proteins in Membrane Fusion. Frontiers in Physiology | wwwfrontiersinorg, 2017. 1: 5. doi:10.3389/fphys. 2017.00005.

[13] John F. W. Keana and Richard B. Roman. Improved Synthesis of $<\mathrm{i}>\mathrm{n}</ \mathrm{i}>$-octyl$\beta$-D-Glucoside: A Nonionic Detergent of Considerable Potential in Membrane Biochemistry. Molecular Membrane Biology, 1978. 1(3-4): 323-327.

[14] S Komura, H Shirotori, P. D Olmsted, and D Andelman. Lateral phase separation in mixtures of lipids and cholesterol. Europhysics Letters (EPL), 2004. 67(2): 321-327.

[15] Aksana A. Labokha, Sabine Gradmann, Steffen Frey, Bastian B. Hülsmann, Henning Urlaub, Marc Baldus, and Dirk Görlich. Systematic analysis of barrierforming FG hydrogels from Xenopus nuclear pore complexes. EMBO Journal, 2013. 32(2): 204-218. doi:10.1038/emboj.2012.302.

[16] Thorsten Lang, Dieter Bruns, Dirk Wenzel, Dietmar Riedel, Phillip Holroyd, Christoph Thiele, and Reinhard Jahn. SNAREs are concentrated in cholesteroldependent clusters that define docking and fusion sites for exocytosis. EMBO Journal, 2001. 20(9): 2202-2213.

[17] Evgenia Leikina, Dilani G. Gamage, Vikram Prasad, Joanna Goykhberg, Michael Crowe, Jiajie Diao, Michael M. Kozlov, Leonid V. Chernomordik, and Douglas P. Millay. Myomaker and Myomerger Work Independently to Control Distinct Steps of Membrane Remodeling during Myoblast Fusion. Developmental Cell, 2018. 46(6): 767-780.e7. doi:10.1016/j.devcel.2018.08.006.

[18] Chao Chen Lin, Jan Seikowski, Angel Pérez-Lara, Reinhard Jahn, Claudia Höbartner, and Peter Jomo Walla. Control of membrane gaps by synaptotagmin-Ca 2+ measured with a novel membrane distance ruler. Nature Communications, 2014. 5: 1-7. doi:10.1038/ncomms6859.

[19] Jennifer Lippincott-Schwartz, Erik Lee Snapp, and Robert D. Phair. The Develop- 
ment and Enhancement of FRAP as a Key Tool for Investigating Protein Dynamics. Biophysical Journal, 2018. 115(7): 1146-1155. doi:10.1016/j.bpj.2018.08.007.

[20] X. Lu. Membrane Fusion Induced by Neuronal SNAREs Transits through Hemifusion. Journal of Biological Chemistry, 2005. 280(34): 30538-30541.

[21] Hana Robson Marsden, Itsuro Tomatsu, and Alexander Kros. Model systems for membrane fusion. Chemical Society Reviews, 2011. 40(3): 1572-1585. doi: 10.1039/C0CS00115E.

[22] Stefanie Marx, Jörg Schilling, Erich Sackmann, and Robijn Bruinsma. Helfrich repulsion and dynamical phase separation of multicomponent lipid bilayers. Physical review letters, 2002. 88(13): 138102.

[23] D. K. McMahon, P. A. Anderson, R. Nassar, J. B. Bunting, Z. Saba, A. E. Oakeley, and N. N. Malouf. C2C12 cells: biophysical, biochemical, and immunocytochemical properties. American Journal of Physiology-Cell Physiology, 1994. doi: 10.1152/ajpcell.1994.266.6.C1795.

[24] James G McNally. Quantitative FRAP in analysis of molecular binding dynamics in vivo. Methods in cell biology, 2008. 85(08): 329-351.

[25] Sutapa Mondal Roy and Munna Sarkar. Membrane fusion induced by small molecules and ions. Journal of lipids, 2011. 2011: 528784.

[26] Yongsoo Park, Javier M. Hernandez, Geert Van Den Bogaart, Saheeb Ahmed, Matthew Holt, Dietmar Riedel, and Reinhard Jahn. Controlling synaptotagmin activity by electrostatic screening. Nature Structural and Molecular Biology, 2012. 19(10): 991-999. doi:10.1038/nsmb.2375.

[27] Ángel Pérez-Lara, Anusa Thapa, Sarah B Nyenhuis, David A Nyenhuis, Partho Halder, Michael Tietzel, Kai Tittmann, David S Cafiso, and Reinhard Jahn. PtdInsP2 and PtdSer cooperate to trap synaptotagmin-1 to the plasma membrane in the presence of calcium. eLife, 2016. 5: 1-22. doi:10.7554/elife.15886.

[28] STEPHEN PERRETT, MICHAEL GOLDING, and W. PATRICK WILLIAMS. A simple method for the preparation of liposomes for pharmaceutical applications: Characterization of the liposomes. Journal of Pharmacy and Pharmacology, 1991. 43(3): 154-161. doi:https://doi.org/10.1111/j.2042-7158.1991.tb06657.x.

[29] Robert D Phair, Stanislaw A Gorski, and Tom Misteli. Measurement of Dynamic 
Protein Binding to Chromatin In Vivo, Using Photobleaching Microscopy. Methods in Enzymology, 2003. 375(2000): 393-414.

[30] Saravanan Rajan, Huan Chu Pham Dang, Haig Djambazian, Harry Zuzan, Yaroslav Fedyshyn, Troy Ketela, Jason Moffat, Thomas J. Hudson, and Rob Sladek. Analysis of early C2C12 myogenesis identifies stably and differentially expressed transcriptional regulators whose knock-down inhibits myoblast differentiation. Physiol Genomics, 2012. 44(2): 183-197. doi:10.1152/physiolgenomics.00093.2011.

[31] Jeffrey Rohrbough and Kendal Broadie. Lipid regulation of the synaptic vesicle cycle. Nature Reviews Neuroscience, 2005. 6(2): 139-150.

[32] M Sass, W Pohle, C Selle, M H J Koch, K Wolfrum, and J Lo. Chain fluidity and phase behaviour of phospholipids as revealed by FTIR and sum-frequency spectroscopy. Journal of Molecular Structure, 1999. 481: 407-411.

[33] Wayne B. Scott, Jennifer E. Stevens, and S A Binder-Macleod. Human skeletal muscle fiber type classifications., 2001.

[34] B SPRAGUE and J MCNALLY. FRAP analysis of binding: proper and fitting. Trends in Cell Biology, 2005. 15(2): 84-91.

[35] David Yaffe and Saxel Ora. Serial passaging of myogenic cells isolated from dystrophic mouse muscle. Nature, 1977. 270(5639): 725-727.

[36] Tae-Young Yoon, Burak Okumus, Fan Zhang, Yeon-Kyun Shin, and Taekjip Ha. Multiple intermediates in SNARE-induced membrane fusion. Proceedings of the National Academy of Sciences of the United States of America, 2006. 103(52): 1973119736. 


\section{CHAPTER 4}

\section{MECHANICAL CHARACTERIZATION OF PHASE SEPARATED DROPLETS AND ITS IMPLICATION ON NUCLEAR TRANSPORT}

Research has been increasing that the formation of membrane-less compartments in cells underlie the liquid-liquid phase separation (LLPS) [2, 33, 62]. This has motivated significant efforts to identify biomolecular condensates' function in normal cells and their involvement in contexts ranging from tumor to age-related diseases [26]. The list of cell compartments believed to be created by the LLPS mechanism is growing increasingly and affects various cell functions [41]. In addition to the formation of membrane-less organelles, other subcellular structures are often formed through LLPS and share common underlying interactions and physical properties. These structures include, for example, heterochromatin, the transport channel in the nuclear pore complex, and cell membrane receptor clusters [19, 26]. Researchers have also obtained insights into the molecular basis of disease through the study of these condensates [7]. Early examples of research in this field focused on the effect of, for example, stress granules on cell survival and their contribution to the progression of amyotrophic lateral sclerosis (ALS) [26, 54]. As macromolecule solutions, such as proteins or nucleic acids, undergo LLPS, they condense into a dense phase that resembles liquid droplets, and this dense phase coexists with a dilute phase. The driving force behind LLPS is the exchange of macromolecule-water interactions for macromolecule-macromolecule and water-water interactions under conditions which are energetically favorable [33], as depicted in Figure 4.1 A. Principles on the thermodynamic driving forces of LLPS and the framework for how multivalent interactions of polymers affect the formation have been discussed widely [62]. Significantly, whether a solution undergoes phase separation depends heavily on the concentration and identity of the macromolecules in solution, as well as on other environmental factors, including temperature, salt form and concentration, $\mathrm{pH}$, and the volume excluded by other macromolecules [22, 33, 46]. 


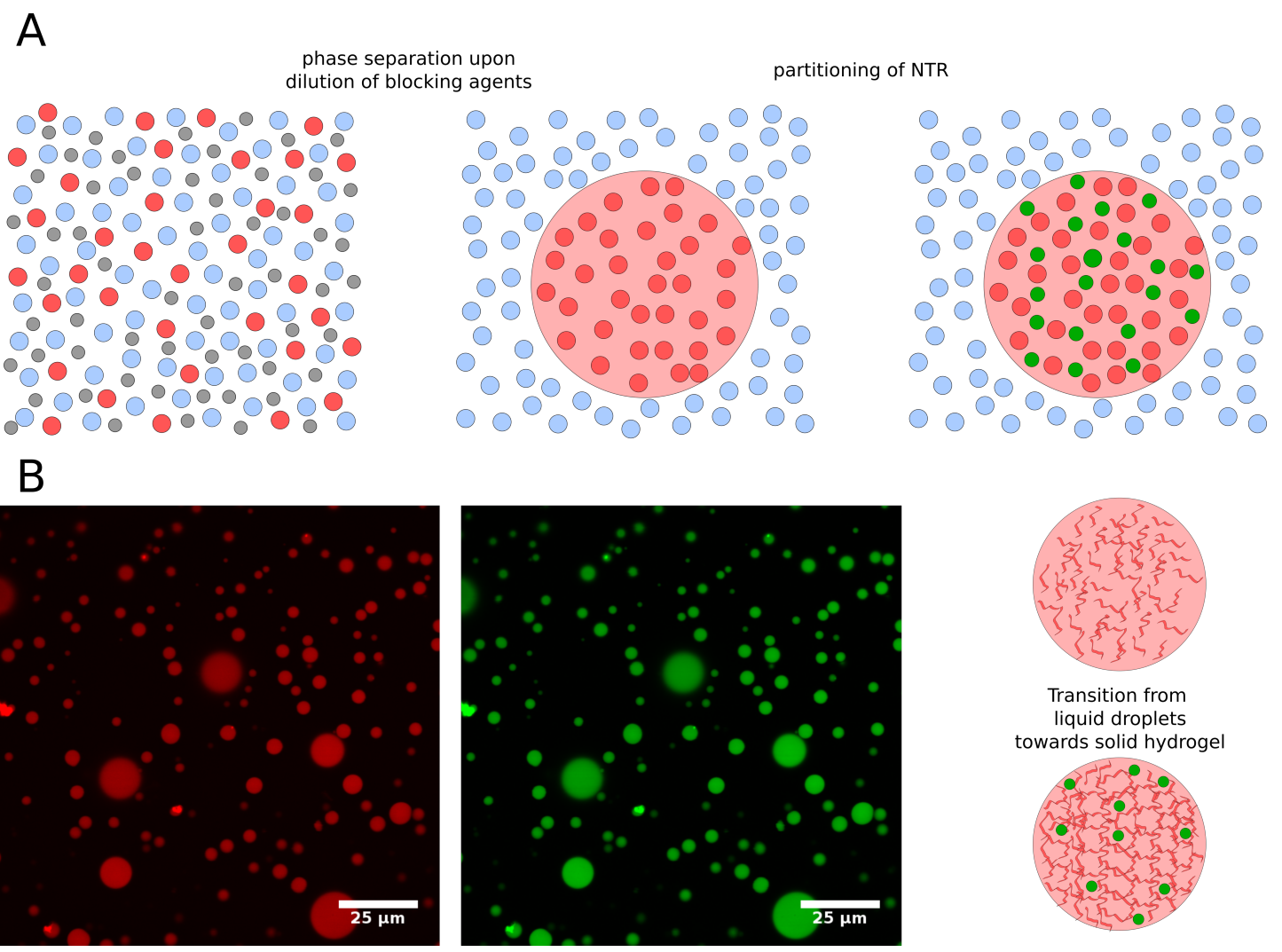

Figure 4.1: A: Schematic illustration of liquid-liquid phase-separation into assemblies of protein droplets (A) and the partitioning of $r$ NTR2 into MacNup98A FG-particle modeled as a hydrogel (B). B: Fluorescence image of the self-assembled FG-particle after dilution of blocking agent (Guanidinium chloride, $4 \mathrm{M} \mathrm{Gua-HCl}$ ). Shown are fluorescence images of the self-assembled FG-particle with incorporated nuclear transport receptor: Alexa 488-labeled hsNTF2 (green, right) and Alexa 647-labeled FG protein (red, right). Images were taken 15 min after mixing FG-protein and NTR2 protein. After dilution $5-25 \mu \mathrm{m}$ protein droplets sediment towards the bottom surface ( $\approx 30 \mathrm{~min}$ ). Scale bar $25 \mu \mathrm{m}$.

Assemblies may fuse or coalesce, which are liquids' typical properties and determined by their surface tension [65]. If the dense phases have liquid-like properties, the polymer molecules are always mobile in the dense phase [33, 62]. The creation of condensed phases by LLPS is also reversible. However, condensates can again undergo more transformations, e.g., gel transitions, mainly if they occur under conditions deep within a two-phase regime. In literature, there can be a further transition to hydrogels as formed by amyloid-like fibers, which are formed irreversibly or require a change in high salt or denaturant conditions to dissolve [1, 7]. In consideration of these potential transformations, LLPS does not have to resolve in liquid, readily fusing assemblies; instead, biomolecular condensates may follow a range of material properties in both physiological and pathological contexts [41]. In vitro phase-separation assays have been commonly used in the study of intrinsically disordered proteins [22]. Intrinsically 
disordered FG domains for example range from 200 to 600 aa, and contain up to 50 motifs [40, 58]. Regions comprised of FG motifs are also varied in length and sequence [30, 36, 51]. As phase separation happens above a critical concentration, FG-Nup's condensed solution emerges from a denaturing buffer that is created through quick dilution into a physiological buffer. Similarly, FG-Nup hydrogels were formed either from lyophilized powder or by rapid dilution of condensed denatured solution [61, 66].

The interaction properties between these hydrogel particles and different cargo proteins have gained particular attention. Many experiments on FG-Nup hydrogel particles have shown that they are rapidly enriched with signal-bearing cargoes recognized by nuclear transport receptors such as Importin- $\beta$ in facilitated import [5]. Inert loads such as dextran $(70 \mathrm{kDa})$ or mCherry without an NTR or Importin- $\beta$ binding domain are excluded from these particles [38, 47]. Hydrogel particles doped with fluorescent FG-Nups did not recover in FRAP experiments when bleached, showing reduced mobility in line with the development of a tough FG-Nup gel particle hydrogel state [61]. This permeability barrier effect was considered by hypothesizing the existence of a mesh structure that eventually translates into mechanical stability through creating a tough hydrogel [38]. Here we propose an approach to describe these phase-separated droplets' hydrogel state and derive elastic and poroelastic parameters from deducing mechanical results and their implications on nuclear transport mechanisms by AFM colloidal probe indentation experiments. Even with modern high-resolution methods it is currently not available to display delicate structures of nuclear pore complexes and the arrangement of the underlying network structures in the presence and absence of NTR [3, 63]. For this purpose, we try to find out the mesh size of these structures mechanically.

As introduced, the difference between an eukaryotic and a prokaryotic cell is the existence of a nucleus that spatially separates distinct cellular processes such as transcription and translation. One particular question of interest is how do the cytoplasm and the nucleus interact and bi-directionally exchange molecules?

The nucleus is surrounded by a double lipid bilayer perforated with nuclear pore complexes (NPCs). All transport must, in principle, take place via the NPCs. Tiny molecules up to 60 kilodaltons can move freely through the NPCs, but larger molecules need transport receptors to allow their transportation through the NPCs. NPC are large, barrel-like protein structures composed of around 30 different proteins, each present in eight copies per NPC with an eight-fold symmetry structure [34, 43]. Phenylalanineglycine-rich (FG) repeats are found in a subset of nuclear pore proteins, nucleoporins (Nups), which are essential for controlling nuclear transport. These FG Nups are special 
in that they are structurally flexible, and they interact with other Nups' FG repeats. The cargo carriers bind reversibly to FG Nups during facilitated transport, import receptors or importins to pass from the cytoplasm to the nucleus. Conversely, cargo from the nucleus to the cytoplasm is carried by export receptors or exportins. Valuable insights into nuclear transport have been provided by fluorescence microscopy combined with kinetic analyses of different transport receptor-bound cargos. While almost everyone agrees that FG Nups form a barrier that enables specific molecules to travel through the NPC selectively, there are several hypotheses about how exactly FG Nups interact with transport receptor-bound cargos to travel through the NPCs [14, 66].

There are four major models for how in the NPCs the FG Nups mediate selective barrier function [15]:

(a) Hydrogel model [29, 32]: FG Nups interact weakly to form a gel in which transport receptor factors and their cargo travels through binding to FG Nups and dissolving FG interactions.

(b) Polymer brush model [63, 69]: FG Nups collapse after being bound by transport factors to form a sheet along the surface of a cylindric channel. This matrix is impenetrable for inert macromolecules but permeable to transport factors. Inert molecules are being pushed away from the central channel.

(c) Reduction in dimensionality model [55, 60]: FG-repeats filaments diffuse along their tether, while other molecules are removed from this area. Transport factors are carried along by binding to the FG Nups and can also serve as a molecular brush that collapses until transport receptors are bound to other molecules.

(d) Trees and bushes in forest model [67]: FG Nups form two types of disordered filaments: collapsing coils, which are gel-like, and expanded coils, which are brushlike. Transport factors can move through both configurations, but macromolecules are excluded.

Given a large number of widely discussed models, it seems reasonable to suspect that the underlying structure of these Nup98-LLPS droplets is foreign. It is not known whether this is a hydrogel, liquid crystals, a complex fluid or soft glassy material. But in this work, we assume that the hydrogel model is best applicable for a mechanical investigation. It has been acknowledged for more than a decade that the FG repeat domain phase-separated into stable hydrogels. Importantly, these gels fully reproduce the NPC permeability barrier properties; i.e., they exclude inert macromolecules and are rapidly penetrated by NTR-cargo complexes [28]. In general, Hydrogels are three- 
dimensional networks of hydrophilic, cross-linked polymer chains capable of absorbing and holding significant water volumes [10,67]. Depending on the production technique, hydrogels can be produced in bulk, thin films, or flakes. Hydrogel particles in the range of $100 \mathrm{~nm}$ to $100 \mu \mathrm{m}$ are referred to as microgels. They can be subdivided into two distinct classes: colloidal gels on a sub-micrometer scale and non-colloid gels on a micrometer scale and beyond [31]. Microgels have essential benefits due to their small dimensions, such as a very high surface-to-volume ratio and effective mass transfer to and from the polymer matrix, but they are challenging to address mechanically. Protein solutions in vitro can be phase-separated to form protein-dense droplets. Such droplets can serve as liquids. In some instances, though, these liquid-like compounds' dynamics slow down over time and become more solid-like as they build tight water-saturated networks. 
In this context, we use AFM and colloidal probes to characterize these hydrogel spheres' mechanical properties on the one hand and draw conclusions on the structure of the underlying network on the other. In particular, the latter is intended to investigate the structure and function of nuclear pore complexes and FG gels as a model system.

The phase-separated hydrogel spheres, which are labeled with the fluorescent dye Alexa-647 (Fig. 4.1 B) are formed from the dilution of the stock solution and the washing out of $4 \mathrm{M}$ guanidinium $\mathrm{HCl}$. The particles sediment on a collagen-I coated glass surface and are examined with the AFM cantilevers mechanical response. The typical way of looking at it is microscopic incident light microscopy. In the incident light experiment, as shown in 4.2 , the spheres appear round and symmetrical. The cantilever is positioned in the center of these droplets in a piezo-controlled manner. From this centered position, an area of $1-2 \mu \mathrm{m}^{2}$ is scanned at points. The sample is being indented in the $z$-direction with an indentation depth of approximately $1 \mu \mathrm{m}$. This is shown in Fig. 4.2 C and D from the side-view. The side experiment shows that the spheres wet the surface, resulting in a half-capped spherical curvature. Therefore, we model the investigation as a half-capped sphere with radius $R$, which is pressed in by a round probe sphere $\left(R_{p}\right)$ with Hertz contact geometry.

This chapter describes the phase-separated material's mechanical properties and how those are linked to its internal structure. We show the Young Moduli under native buffer conditions and with a variation of the sodium chloride concentration. When the droplets are indented, a time-dependent deformation can be observed, which we analyze with a poroelastic model in order to obtain a mesh size of the underlying network from the combination of the rigidity of the material. This size is then to be correlated and confirmed with the results from experiments of dissolution of singlemolecule bonds. Figure 4.3 clarifies this approach schematically. The investigation is divided into three segments, of which the results are presented and discussed in the following subsections. 


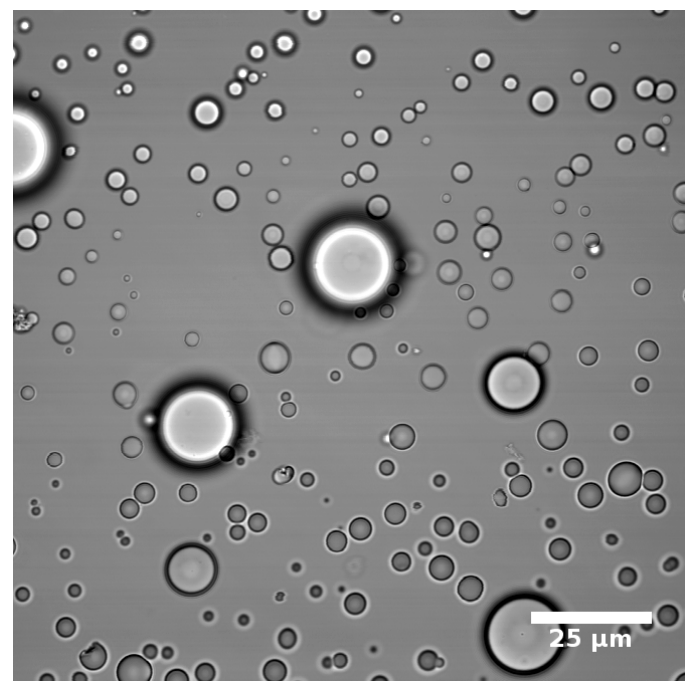

(a) bright-field illumination

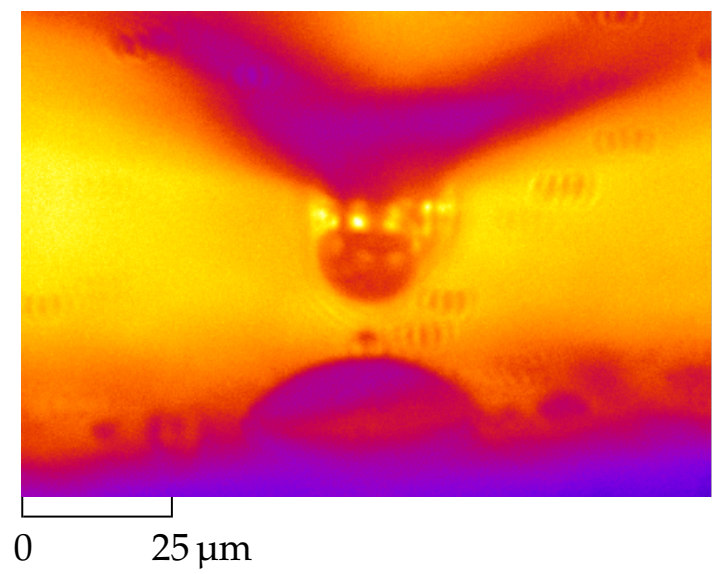

(c) AFM sideview experiment

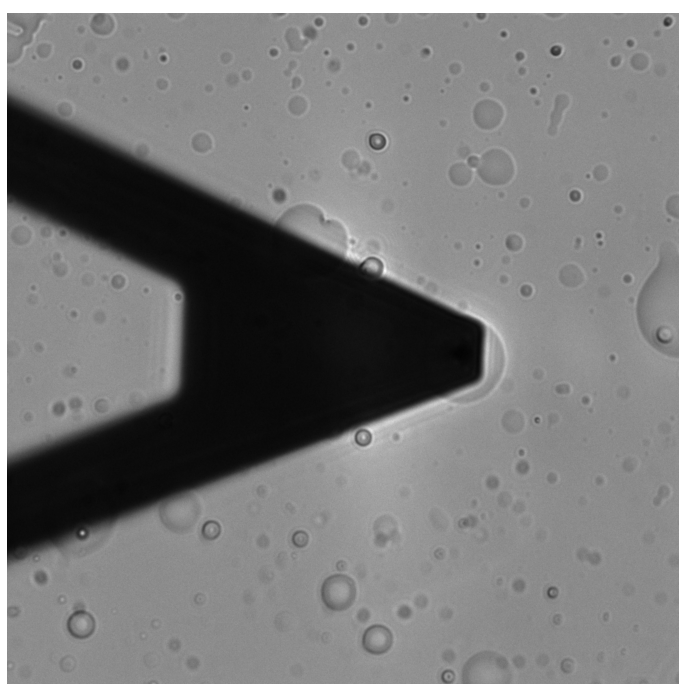

(b) AFM topview experiment

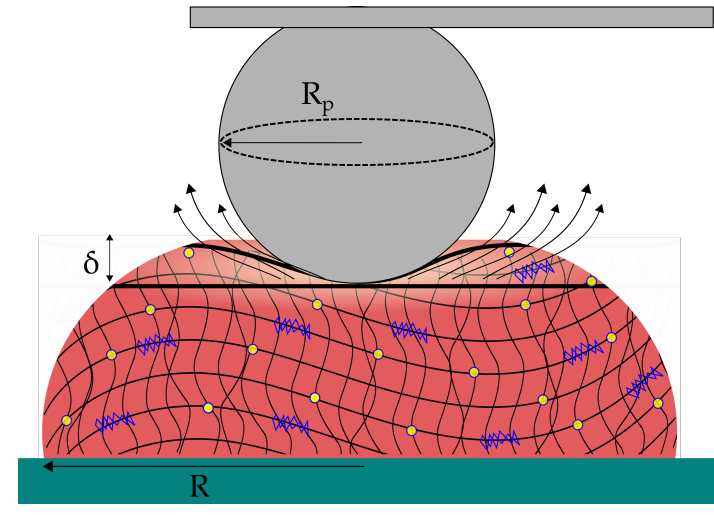

(d) schematic experiment

Figure 4.2: Mechanical approach by AFM with colloidal probe force spectroscopy. A: Transmission image of the self-assembled FG-particle. B-C: Fluorescence images of the self-assembled FG-particle with incorporated nuclear transport receptor. Images were taken 40 min after mixing FG-protein and NTR2 protein. After dilution 5-25 $\mu$ protein droplets sediment towards the bottom surface. Scale bar $25 \mu \mathrm{m}$. B: AFM-experiment equipped with a sideview cantilever holder. A $15 \mu \mathrm{m}$ bead indenter mounted on a V-shaped cantilever in contact with the target FG-particle adhered on glass. B and C: Shown are top- and sideview experiments. D: Schematic showing a typical experimental setup for the indentation of a FG-particle using atomic force microscopy. A particle of radius $\mathrm{R}$ is indented by a distance $\delta$ with a probe of radius $\mathrm{R}_{\mathrm{p}}$ attached to the AFM cantilever. 
A

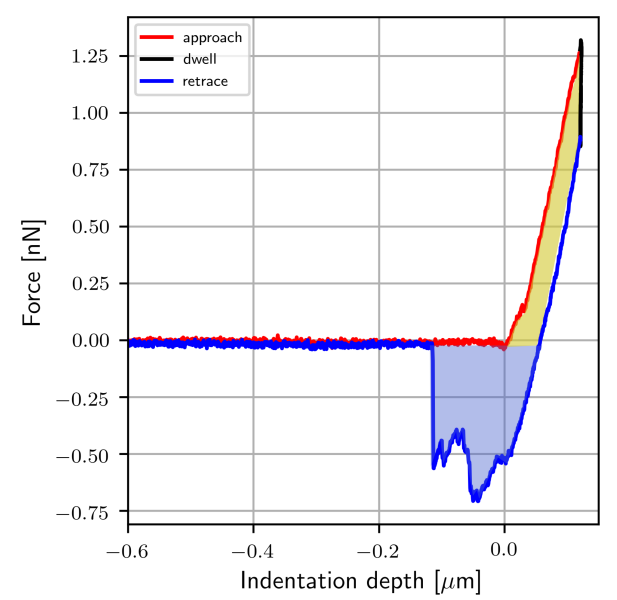

B

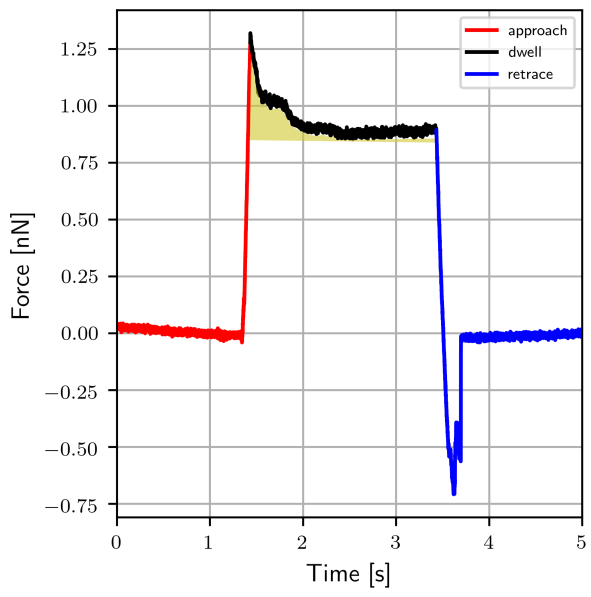

C

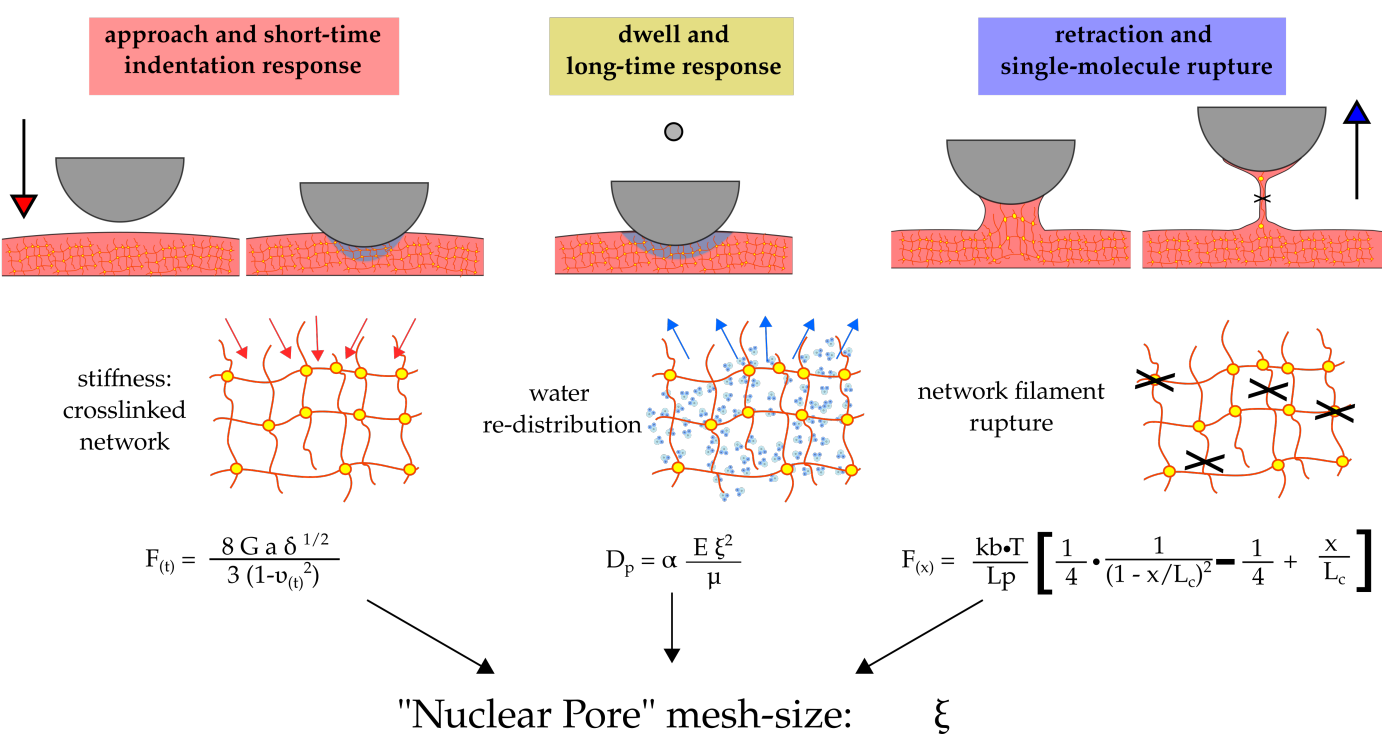

Figure 4.3: Mechanical response upon indentation of FG-Hydrogel particle with a diameter of $20 \mu \mathrm{m}$. A: Force-indendation-curve with associated AFM-sequence: approach, dwell and retraction. Work of adhesion is shown in blue. Peaks correspond to filament and intermolecular bond rupture of the hydrogel network. B: Measured force and indentation depth as functions of time obtained from AFM using a colloidal probe. Force-Time-Curve shows time dependent relaxation of force during dwell. Dwell time fits to a stretched exponential model of poroelastic relaxation with a characteristic relaxation time. Water is being excluded from FG-Hydrogel in the contact area underneath the spherical glass probe. $\mathrm{C}$ : Schematic representation of contact mechanics of the underlying microstructure upon deformation. A poroelastic FG-particle of radius $R$ is indented by a distance $\delta$ with a probe of radius $R_{p}$ attached to the AFM cantilever. The probe is held against the particle for a period of time, and the resultant applied force $F(t)$ is measured. The poroelastic particle is characterised by the shear modulus $G$, the drained Poisson's ratio $v$, and the diffusivity $D$ of re-distributing water content. Symmetric indentation of a poroelastic particle by spherical indenters Hertzian geometry is assumed. 


\subsection{Mechanical response upon indentation}

The atomic force microscope is beneficial for probing the mechanical response of samples like the elastic modulus of proteins. Cantilevers serve as soft nanoindenters allowing local sample testing of biological tissues like cells or bones [18]. Young's modulus for different biological materials can address as low as $100 \mathrm{~Pa}$ and as high as up to $10 \mathrm{GPa}$ [37, 70]. Young modulus is a mechanical property that measures the tensile strength of a solid material. Tensile stress quantifies the relationship between force and strain during elongation. When you bend a solid material, it elastically deforms. It is reversible as the material returns to its original shape after the load is removed. Studies have shown that for example, collagen gel stiffness increased with concentration, $\mathrm{pH}$, or the crosslinking procedure's temperature [4]. The mechanical properties of collagen gels vary due to changing polymerization conditions [16]. To address how a phase of FG supplies the central channel of the nuclear pore and what rigidity the phase transition materials provide, we perform atomic force microscopy measurements and extraction of Young's moduli of the FG microgels. Experiments were performed at room temperature and under physiological buffer conditions, in the presence and absence of DTT, and under $\mathrm{NaCl}$ variation (0, 50, 300, $600 \mathrm{mM}$; see Chapter 3 Table 3.1). Adjustment of salt concentration might affect only the permeability of adsorbed phase. The mean diameter of FG hydrogel particles prepared was $33 \pm 5 \mu \mathrm{m}$. We attempted that microgels droplets that we approached had a narrow size distribution. The droplets had a well-defined, perfect circular shape.

The results from this section are shown in Table 4.1 and Figure 4.4. In general, the measurements have shown that the hydrogel particles' mechanical response as being indented by the cantilever is very stiff at $112 \pm 50 \mathrm{kPa}$ compared to, for example, cells [37]. In general, the E-modules are widely distributed and mostly mechanically dense. Different distributions from the data set obtained show different characteristics, indicating that the mechanical properties change during the measurement. This aspect becomes more important for the time-dependent consideration of hydrogels' mechanical properties in the later chapter. The distribution of Figure 4.4 A shows experiments using the addition of $2 \mathrm{mM}$ dithiothreitol (DTT) a fraction with soft characteristics (I: 10-25 $\mathrm{kPa}$ ), which is overlapped by the harder fraction (I: $135 \mathrm{kPa}$ ). On average, these are $134 \pm 60 \mathrm{kPa}$ hard. The experiment in Figure 4.4 A $300 \mathrm{mM}$, on the other hand, shows a very soft mechanical response of $38 \pm 31 \mathrm{kPa}$ with a left-skewed distribution. Due to the skewed distribution, the superposition of soft and hard fractions, and the fact that each AFM experiment is recorded as it progresses in time, we follow the idea that the hydrogel samples are hardened during the experiment, which would explain the wide distribution of values. 


\begin{tabular}{c|cccccc}
$E_{\text {Young }}[\mathrm{kPA}]$ & $\mathrm{w} / \mathrm{DTT}$ & $\mathrm{w} / \mathrm{o}$ DTT & $0 \mathrm{mM}$ & $50 \mathrm{mM}$ & $300 \mathrm{mM}$ & $600 \mathrm{mM}$ \\
\hline \hline (Mean +- SD) & $134 \pm 60$ & $176 \pm 50$ & $103 \pm 60$ & $136 \pm 67$ & $38 \pm 31$ & $89 \pm 32$ \\
& & & & & & \\
\hline
\end{tabular}

Table 4.1: Young Moduli from AFM-based elasticity measurements carried out on FG-Hydrogel droplets with a mean diameter of $33 \pm 5 \mu \mathrm{m}$ under variation of $\mathrm{NaCl}$ concentration. Force distance curves were obtained for maximum force setpoint of $1 \mathrm{nN}$ and an approach velocity of $1 \mu \mathrm{m} \mathrm{s}^{-1}$. Measurements were performed at RT in Tris buffer and variation of $\mathrm{NaCl}(50 \mathrm{mM}$ Tris, $\mathrm{pH}=7.5)$.

It was a matter of interest that the sodium chloride concentration could influence the permeability of the FG phase. By varying the sodium chloride concentration, we could not determine any systematic changes in the mechanical properties and osmotic or isoelectric regulation of the underlying network structure. Interestingly, the distribution and the experimental observations differ from each other. The distribution of $0 \mathrm{mM}$ and $600 \mathrm{mM}$ hardly differ from their central position. Both are relatively soft $\approx 100 \mathrm{kPa}$. The $50 \mathrm{mM}$ distribution again shows a hard $(>100 \mathrm{kPa})$ and a soft fraction $(<100 \mathrm{kPa})$. For the sake of completeness, it should be mentioned that there were a fraction of experiments in a period less than 30 minutes after the LLPS in which the cantilever could not be approached to the sample or the modulus of elasticity was exceptionally soft $(\approx 0.5-2 \mathrm{kPA})$. It is known from the literature that LLPS materials initially have liquid properties [12, 19]. These can then assumely transform into a gel-like state, which is then usually followed by a solid-state in a pathological context [54]. It was also observed that the FG phase is neither liquid nor stable as a solid. We supported this observation and assumed that the hydrogel state is in a range between 10-150 kPa. The time dependency of the measurements carried out is another effect that must be taken into account. During the dwell time between the cantilever's approach and retraction, a time-dependent relaxation of the force response is observed (Fig. 4.3 A and B, yellow). Considering the state of the hydrogel, we assume that this relaxation is because the water from the swollen hydrogel is redistributed to the cantilever's contact surface under the influence of the pressure applied. The redistribution is strongly dependent on the indentation speed when the cantilever and the sample approach. This dependency is the direct basis of a poroelastic viewpoint. If the sample is slowly indented, the water content initially distributes very quickly with the given viscosity of water and depending on the underlying network density, so that the apparent stiffness of the network is relatively high. A dried-out network is mainly deformed. The Young module is big. If, on the other hand, the index is very fast with approach speeds $>25 \mu \mathrm{m} \mathrm{s}^{-1}$, the network is mainly swollen with water, and the mechanical response is soft. 

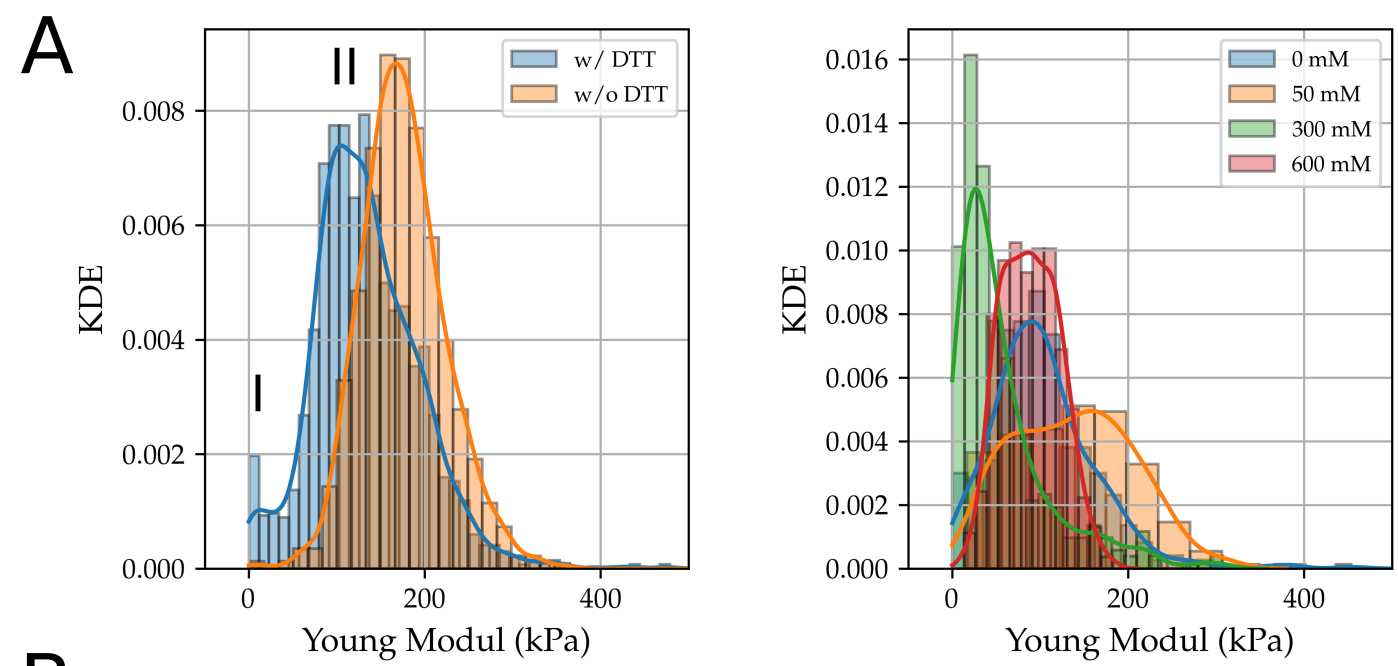

B
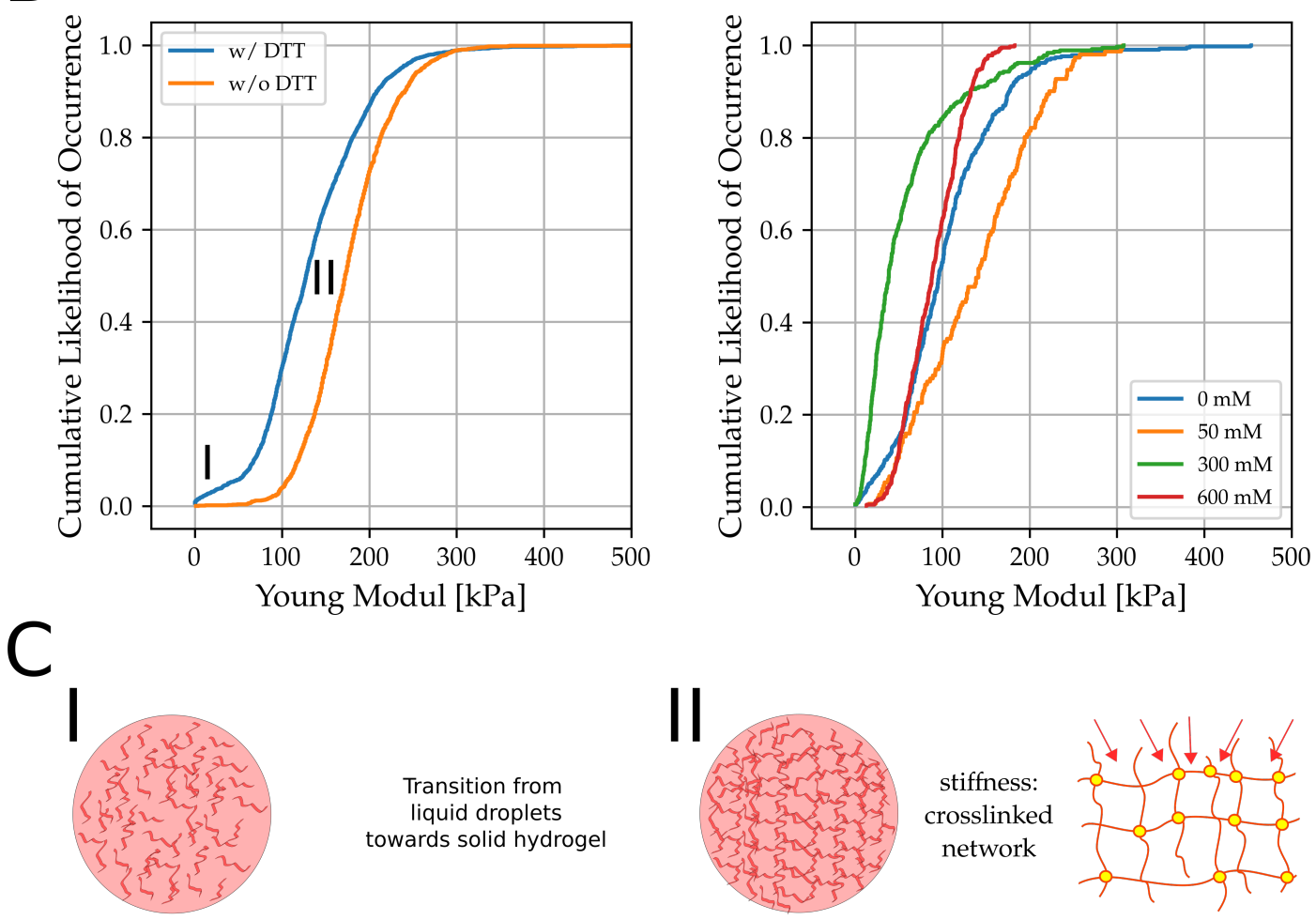

Figure 4.4: AFM-based elasticity measurements carried out on FG-Hydrogel droplets with a mean diameter of $33 \pm 5 \mu \mathrm{m}$. A: Histograms showing the distribution of Young's modulus of FG-Hydrogel determined from the Hertz fit of 1000 force-indentation curves perfomed under different buffer conditions. B: Cumulative histograms derived from A. C: Schematic interpretation of soft (I) and stiff (II) response corresponding to hydrogel and solid states as observe in A. Measurements were performed at RT with assay buffer ( $50 \mathrm{mM}$ Tris, $150 \mathrm{mM} \mathrm{NaCl}, \mathrm{pH}=7.5$ ) in presence and absence of $2 \mathrm{mM}$ DTT (A).

Hydrogels can be used as scaffolds in tissue engineering. Hydrogels are soft and wet materials that contain $50-80 \%$ water. Conventionally produced hydrogels have low Young's modulus (1-200 kPa) [6, 10, 70], low tensile and compression strength, and 
low fracture energy (10 joules per square meter) [25, 57]. Hydrogels are useful in most biomedical and other applications due to their liquid-like properties. We need to tune the mechanical properties and physical makeup of our hydrogels in a controllable way. In literature, crosslinked acrylamide and other crosslinked polyacrylamide networks are discussed [9].

\begin{tabular}{c|ccc}
$\begin{array}{c}\text { (Mean } \pm \text { SD) } \\
\text { w/o } \\
\mathrm{w} / \mathrm{NTF} 2\end{array}$ & $22 \mu \mathrm{m}$ & $6.5 \mu \mathrm{m}$ & $15 \mu \mathrm{m}$ \\
\hline \hline \multirow{\delta}{*}[\mu\mathrm{m}]{} & $0.407 \pm 0.097$ & $1.11 \pm 0.40$ & $0.83 \pm 0.27$ \\
& $1.09 \pm 0.30$ & $1.17 \pm 0.39$ & $0.48 \pm 0.17$ \\
$\tau_{1}$, & $0.71 \pm 0.05$, & $0.63 \pm 0.24$, & $0.91 \pm 0.23$, \\
$\tau_{2}[s]$ & $0.069 \pm 0.016$ & $0.043 \pm 0.016$ & $0.076 \pm 0.045$ \\
& $0.56 \pm 0.29$, & $0.53 \pm 0.05$, & $0.94 \pm 0.35$, \\
& $0.048 \pm 0.071$ & $0.040 \pm 0.007$ & $0.063 \pm 0.05$ \\
& $14.29 \pm 4.62$ & $70.12 \pm 28.41$ & $23.90 \pm 9.08$ \\
$D_{\text {pore }}\left[\mu m^{2} / s\right]$ & $88.11 \pm 28.18$ & $82.39 \pm 28.53$ & $18.23 \pm 8.96$ \\
\hline & & &
\end{tabular}

Table 4.2: AFM-based poro-elasticity and relaxation and the influence of NTR (NTF2) carried out on FG-Hydrogel droplets with a mean diameter of $50 \mu \mathrm{m}$ under variation of AFM-tip size: 2, 6.5, and $15 \mu \mathrm{m}$ diameter. Shown are indentation depth $\delta, 1$ st and 2nd relaxation time from double exponential fitting $\tau_{1,2}$, and Diffusion coefficients $D_{\text {poro }}$ from Poroelastic fitting of dwell force-time relaxation. Force distance curves were obtained at maximum force setpoint of 7-8 $\mathrm{nN}$ for a target indentation depth of $1 \mu$ at an approach velocity of $50 \mu / \mathrm{s}$. Measurements were performed at RT with assay buffer (50 mM Tris, $150 \mathrm{mM} \mathrm{NaCl}, \mathrm{pH}=7.5$ )

For the further extraction of the mesh-size, a poroelastic model is used, which requires the mechanical model of a sponge saturated with water. FG hydrogels with high approach speeds were examined in the following, and the time-dependent force relaxation was characterized. The description is based on the indentation depth, the relaxation time between short time and long time response, and the poroelastic diffusion coefficient (Table 4.2 and Figure 4.5). On the one hand, the indenter's contact geometry is considered here, as we vary its diameter, and on the other hand, the influence of NTF2 ( $\approx 30 \mathrm{kDa}$ transport receptor that is known to interact with the FG domain [66]). The latter is described in more detail in the following section for two indenter sizes.

The relaxation times, which were determined by double exponential fit, always show a fast and slow relaxation. These are attributable to the rearrangement of water molecules on the one hand and a restructuring of the network on the other [11, 44, 45]. The mechanical response and the apparent Young's modulus mainly determines the poroelastic 
mesh size during rapid deformation. This indicates a type of network density. The poroelastic model is based on the assumption that, with constant indentation depth, our hydrogel's water content is distributed according to its viscosity depending on the pore size [49, 53]. The relaxation process is mostly always the same. However, it is dependent on the pressure applied and thus the contact area of the indenter. For this reason, the force response of Poroelastic relaxation curves is normalized and represented in a standardized way. The time of relaxation is scaled to the diffusion as well as to the contact surface so that after a purely poroelastic process, all curves obtained are subject to a universal behavior - a so-called master curve [23] and are then be fitted with equation 2.26. Figure 4.5 A (superimposition of force-time curves for three indenters, right) clearly shows that this is only partially the case for the FG hydrogel. These deviations can be caused by irreversible deformation of the specimen during the indentation. There is a possibility that this deformation increases the adhesion surface, and thus, the curves are no longer uniformly scaled with $a^{2}$ [9, 17, 50]. Another case would be that the contact area is too small for the poroelastic indentation so that a more solid-like network and not a network saturated with water is indented [52]. The measurement with a small indenter with a diameter of $2 \mu \mathrm{m}$ shows a more rigid elasticity than with the other indenter. The target penetration depth is only half as considerable here with $\approx 0.407 \pm 0.097$. Here the force relaxes more slowly, and the water content diffuses less. The influence of NTF2 on the mechanical behavior of the FG hydrogels was essential for us. Indenter 6.5 and 15, on the other hand, show a relatively good overlap with short relaxation times, which is why these two are used for further discussion of the influence of NTR on permeability. In the presence of NTF2, the FG hydrogels actually show very different mechanical properties than the wt-FG particles without NTF2. In general, these are softer by a factor of 2-3. The relaxation times are also shorter so that the diffusion coefficient is increased. This has the consequence that the hydrogel's pore-size would increase, which will be discussed in the following section. 

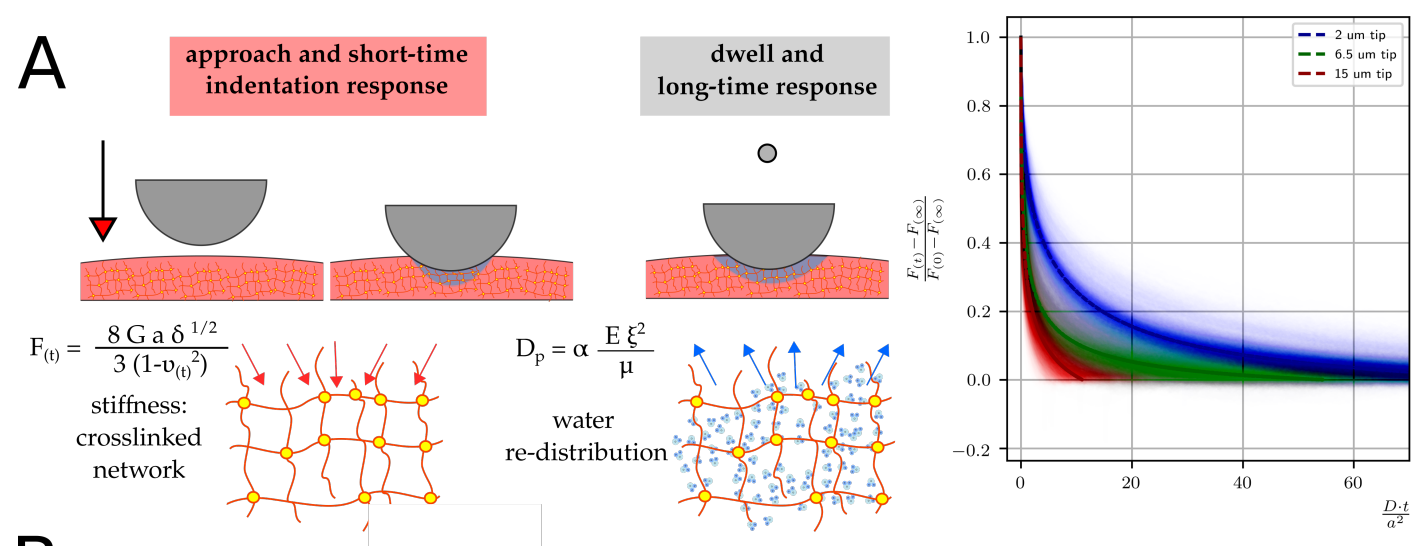

B
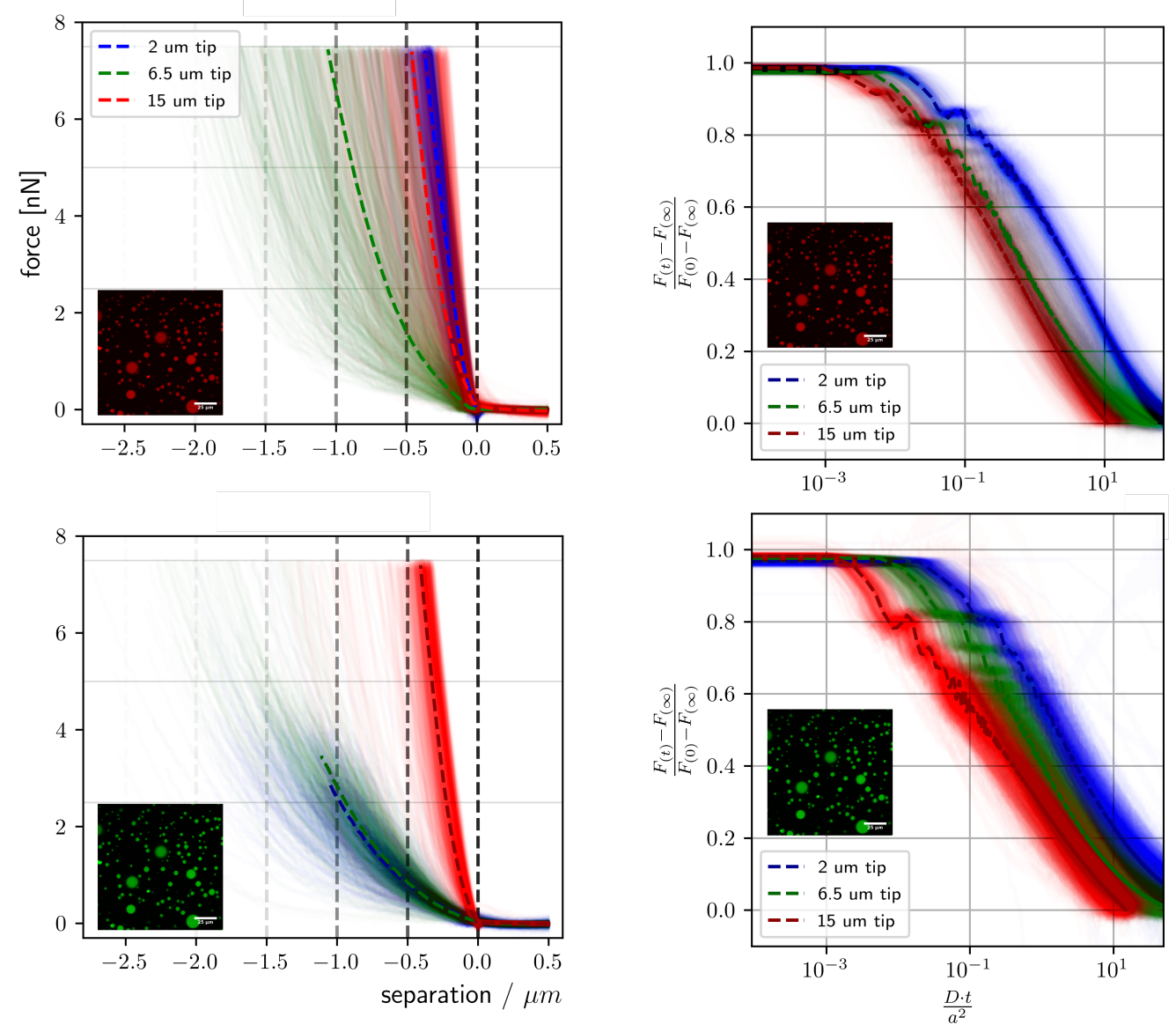

Figure 4.5: AFM-based poro-elasticity measurements and the influence of NTR (NTF2) carried out on FG-Hydrogel droplets with a mean diameter of $50 \mu$ m under variation of AFM-tip size: 2 blue, 6.5 green, and $15 \mu \mathrm{m}$ red diameter. Force-Relaxation times are recorded for a Force Setpoint of $4-8 \mathrm{nN}$ corresponding to a target indentation depth of $\delta \approx 1 \mu \mathrm{m}$. A: schematic representation of poro-elasticity measurements and the corresponding force relaxation curve obtained from dwell response of $3 \mathrm{~s}$ after indentation of the sample with a velocity of $25 \mu \mathrm{m} \mathrm{s}^{-1}$. Shown are superposition of 900 force-distance curves with a median curve (dashed lines). Experiment performed in Assay buffer $(50 \mathrm{mM}$ Tris, $150 \mathrm{mM} \mathrm{NaCl}, \mathrm{pH}=7.4$. B: Influence of NTR on indentation (left) and relaxation (right) of FG-Hydrogel. Force relaxation curves are normalised between short and long-time response and scaled with $D_{p}$ and corresponding indentation contact area $a^{2}$ from spherical indentation depth. Shown is a logarithmic comparison of different tip sizes. 


\subsection{Poroelastic relaxation response and influence of nuclear transport receptor NTF2}

Nuclear transport factor $2(\mathrm{NTF} 2,33 \mathrm{kDa})$ is a small, heterodimeric protein that binds to RanGDP and yeast Nsp1p. NTF2 is a critical component for the efficient transfer of RanGDP [66]. The interaction between NTF2 and RanGDP is crucial for efficient nuclear protein import, and recent work has indicated that a critical role of NTF2 is to mediate the nuclear import of RanGDP. However, the precise mechanism by which NTF2 facilitates Ran's nuclear import is unknown. Although nuclear import rates generally have a positive effect on the nuclear size, NTF2 levels have a significant impact on the nuclear size, despite the NTF2's role in recycling the Ran factor [8, 27]. The poroelastic AFM indentation approach is vital to understand how nuclear "pore "size is regulated.

In this chapter, we compare the poroelastic AFM experiment with indenters of 6.5 and $15 \mu \mathrm{m}$ in diameter in the presence and absence of NTF2. When NTF2 is present, the FG-hydrogels actually have different mechanical properties from the controls. More extensible by a factor of 2-3. The relaxation times are shortened for a higher diffusion coefficient. This is an influence from $70.12 \pm 28.41$ to $82.39 \pm 28.53$ (mean \pm SD) as NTF2 incorporates into the gel. The maximum of the distribution shown in Figure 4.6 shifts from 57.4 to $71.7 \mu \mathrm{m}^{2} / \mathrm{s}$. Because of the proportionality between $E, D_{p}$, and $\xi_{\text {hydrogel }}$, the network stiffness is directly influenced when increasing the hydrogel mesh size and vice versa. In a wider network, water can diffuse more freely, given more interaction by NTF2. The transport receptor softens the gel. Relaxation time remains constant. Non-covalent bonds can cause a range of other interactions. They are thus increasing the mobility of the gel phase while the transporter is present. As a consequence, the presence of the transporter facilitates its own and the incorporation of bigger molecules.

We observe a direct increase in the poroelastic diffusion coefficient due to an enlarged mesh size. Figure 4.6 B shows that as a consequence the maximum of the distribution from $7.7 \mathrm{~nm}( \pm 9.7 \mathrm{~nm}, \mathrm{SD})$ shifts to $18.0 \mathrm{~nm}(20.8 \pm 8.3 \mathrm{~nm}$, median \pm MAD) at presence of the transporter. Both distributions are not normally distributed. The pure Nup98 hydrogel mainly follows a log-normal distribution. Considering a porous structure, this means that there are a large number of small pores in addition to a few large pores. The maximum pore size is approximately $55 \mathrm{~nm}$. In a biological context of the cells nucleus, two Nup98 filaments arranged in parallel would span the core pore and thus form a pore. Calculated downwards and based on the smaller pore size, smaller than $7 \mathrm{~nm}, 7-8$ filaments would be sufficient to build an efficient sieve-like barrier. In the presence of NTF2, this is expanded, which shifts the maximum of the distribution. It 

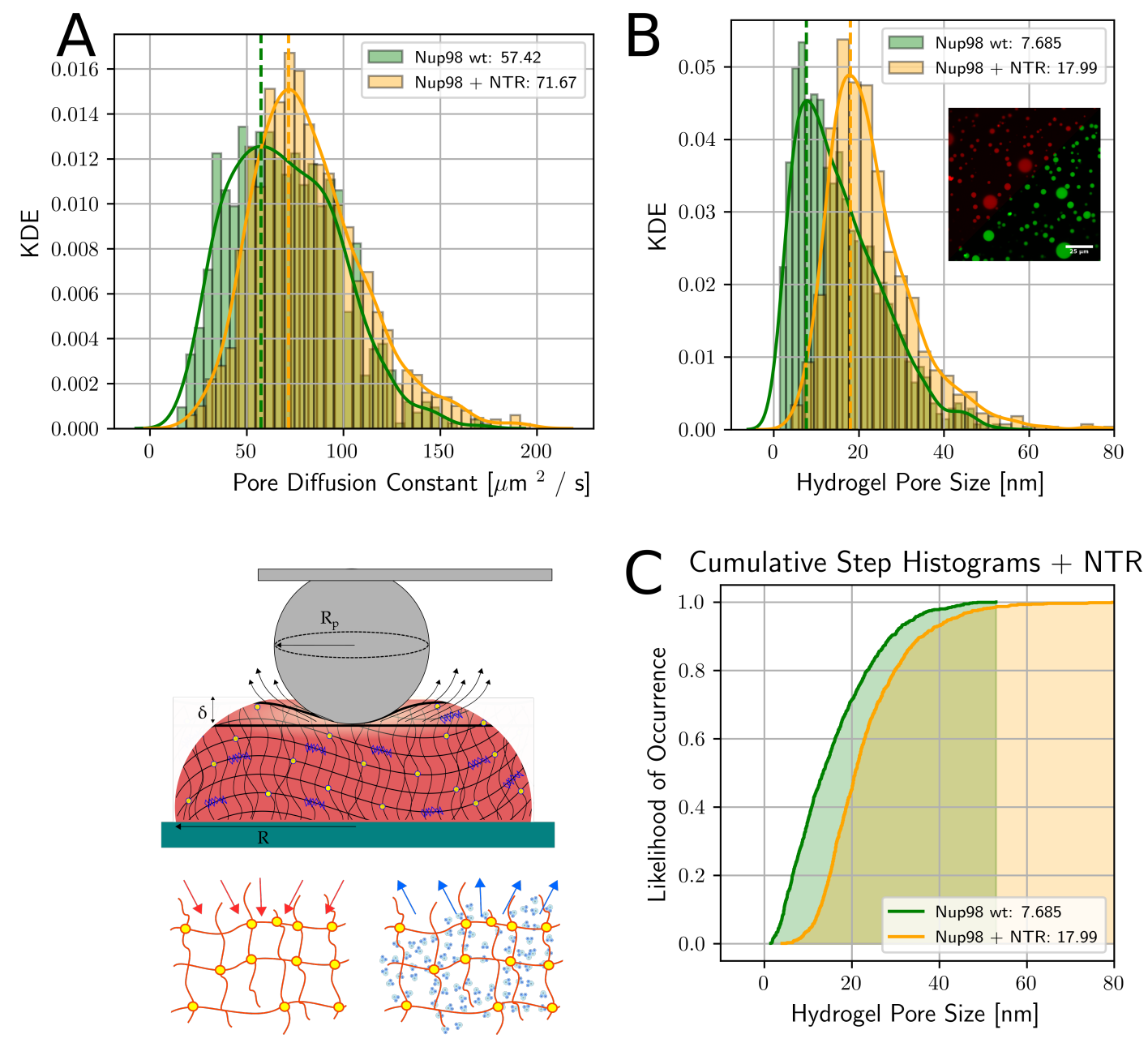

Figure 4.6: Effect of NTF2 on the poroelastic diffusion coefficient and the connected hydrogel pore size. AFM-based poro-elasticity measurements performed with an indenter diameter of $R_{p}=6.5 \mu \mathrm{m}$ and a targe indentation of $\delta=1 \mu \mathrm{m}$. Shown are Nup98-wt in the absence of NTR in green and in the presence of NTR in orange. A: Histograms showing the distribution of Pore diffusion coefficients characterizing the re-distributing water content of the FG-Hydrogel after indentation. B: Histograms showing the distribution of the corresponding hydrogel pore size and an increase in pore diameter in the presence of NTR. C: Cumulative histograms derived from B.

can also be observed that the second distribution tends to follow a normal distribution. From a macroscopic point of view, in the presence of NTF2, interconnections in the network, which has formed tiny pores, are broken, and bigger passages are created. This would also have the consequence that the observed network stiffness decreases and the gel becomes softer. We emphasized that this kind of mechanism might be reversible. However, we see no way to prove this, as we do not see any possibility of removing the transporter from the network.

The further measurement in which the sample was indented with a $15 \mu \mathrm{m}$ tip shows 

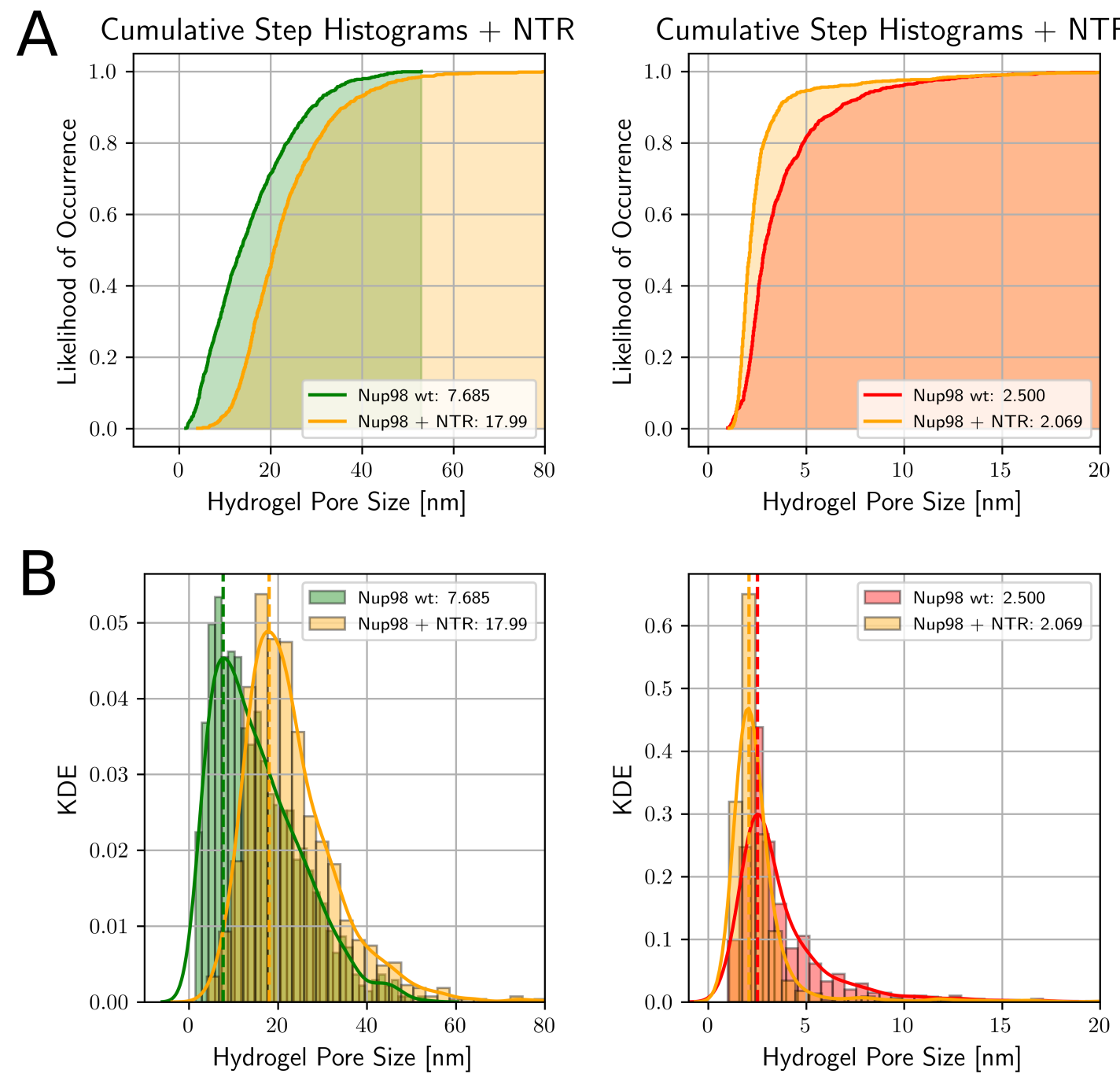

Figure 4.7: Comparison of the effect of NTF2 on the hydrogel pore size for two different indenter sizes corresponding to a soft and a stiff mechanical response upon indentation. AFM-based poro-elasticity measurements performed with an indenter diameter of $R_{p}=6.5 \mu \mathrm{m}$, green and $R_{p}=15 \mu \mathrm{m}$, red. Shown are Nup98-wt in the absence and in the presence of NTR in orange.

a very firm and stiff mechanical response. In each of the poroelastic measures, care was taken to ensure that the first experiment of a consecutive series of experiments was carried out with an indentation depth of $1 \mu \mathrm{m}$. However, as shown above, we observe that the $15 \mu \mathrm{m}$ experiments, in particular, became progressively harder. We, therefore, assume that the sample will age during the measurement. This aging can also be induced mechanically by stress-stiffening. Water is continuously pressed out of the sample without diffusing back into the hydrogel quickly enough. We, therefore, assume an irreversible deformation and a draining network. In practical terms, this would mean that the smallest pore size to be expected would also be $2.5 \mathrm{~nm}$ from a 
log-normal distribution. An influence of NTF2 on such a type of destroyed network could not be observed as shown in Figure 4.7 B. Likewise, these networks did not soften in the presence of the transporter. The adequate pore size decreased slightly even after the incorporation of the NTR. At this point, the author is not aware of any further results in the literature that clearly show whether and which structures the Nup98 assemblies form. For confirmation of a poroelastic network's hypothesis and estimated pore-sizes, single-molecule demolition experiments were carried out and presented in the following.

\subsection{Destruction of the hydrogel network upon dissolution of underlying single molecule interactions}

This section uses AFM and force spectroscopy to investigate the interaction between a cantilever tip and a FG-Hydrogel as the cantilever is retracted from the surface. Mostly force spectroscopy probes the relevant characteristics of single molecules and molecular assemblies [20]. The crux of this method is an analysis of the histograms obtained at different force-loading rates into the force-dependent lifetimes measurable [48]. Classically one interprets the force-dependent lifetimes by deriving a generalization of Bell's formally exact formula within the framework of Kramer's theory [68]. Over the years, mechanical forces are increasingly recognized to play a growing role in biology. Particular on a molecular level. Therefore single-molecule force spectroscopy presents a deceptively simple approach to probing intermolecular interaction by measuring forces needed to pull the molecules apart.

The importance of mechanically stretching single molecules that can change their shape has increased since the first reports on unfolding titin were published [56, 59]. The disordered proteins were assumed to behave as worm-like chains (WLC) for which the stretching force is described [13]. For this problem, one studies at $\mathrm{L}_{C}$ the contour length of the molecule, $k_{B}$ the Boltzmann constant, $\mathrm{T}$ the absolute temperature, and $\mathrm{x}$ the pulling distance. But molecular assemblies like Hydrogels and especially from intrinsically disordered proteins often don't show exclusive single-molecule characteristics. In complex materials, one observes many different alternating effects, including distributions of short and long-lasting bonds. It can therefore be very consuming and challenging to isolate and assign individual events. Many natural materials depend on the interactions between weak and strong bonds and their combination as well. One exciting concept is one of the therefore formed sacrificial bonds which can toughen a material. 
Sacrificial bonds with a hidden length were discovered, with single-molecule force spectroscopy, to function as a mechanical origin for the toughness of natural fibers, composites, and adhesives [24, 25]. The part from the length of a polymer molecule that can be hidden from external forces when working on stretching the molecule isolates it with a sacrificial bond. For stretching a molecule, a force is needed to apply against entropic force and enthalpic force. These bonds and the hidden molecular-lengths in structural molecules and composites greatly increase biomaterials' durability by providing a reversible, molecular-scale energy-dissipation mechanism [21]. In networks of cross-linked molecules, large amounts of length can be hidden from an applied load by a variety of cross-links, including both sacrificial bonds and entire cross-linking molecules. One can think of this as interlocking molecular loops within a network forming different mesh sizes, where water can diffuse through. With AFM singlemolecule spectroscopy, the resulting force spectrum is moderately disordered and complex.

Practically, the rupture distance of a specific adhesion event will depend on the molecule's extended length. In a dense and cross-linked network formed by LLPS, one will observe many bonds and interactions resulting from multiple strands, single filaments, weak and robust cross-links, and single and bulk interactions, as shown in Figure 4.8. Figure 4.8 A shows an exemplary force-distance curve for an extension of the z-piezo driven cantilever from zero to $500 \mathrm{~nm}$. The fitting indicates an application of the WLC-model (see Fig. 4.13 S4). We divided this curve into two segments; first, there is a segment with many small rupture events at high forces up to an extension of $200 \mathrm{~nm}$. Here we observe many peaks belonging to sacrificial bonds systems. We name this region bulk-rupture events. A catenoid between the tip and FG-Particle is formed as shown in Figure 4.8 A I and Figure 4.9 A. This segment is a region where we rupture parts of the whole assembly apart. Secondly, a segment with prominent peaks and WLC characteristics at small and large forces after a tip-sample separation of $200 \mathrm{~nm}$ follows. In Supporting Figure 4.13 S4, we show scatter-plots and the corresponding histograms of persistence and contour length color-coded for the peak-to-peak distance $\Delta L_{C}$ between the peaks of this region. The distribution of persistence length is skewed to values below $0.4 \mathrm{~nm}$, resulting in a relatively stiff mechanical response. Consequently, these peaks belong to multiple filaments or fibers being pulled out of the gel due to a highly cross-linked gel. These can extend up to several micrometers, as shown in Figure $4.8 \mathrm{~B}$ and Figure 4.9 A.

Instead of looking at the single filament mechanics, we focus on the bulk mechanics, where cantilever retractions destroy the entire network assembly. Nanomechanical interactions of phenylalanine-glycine nucleoporins studied by single-molecule force- 

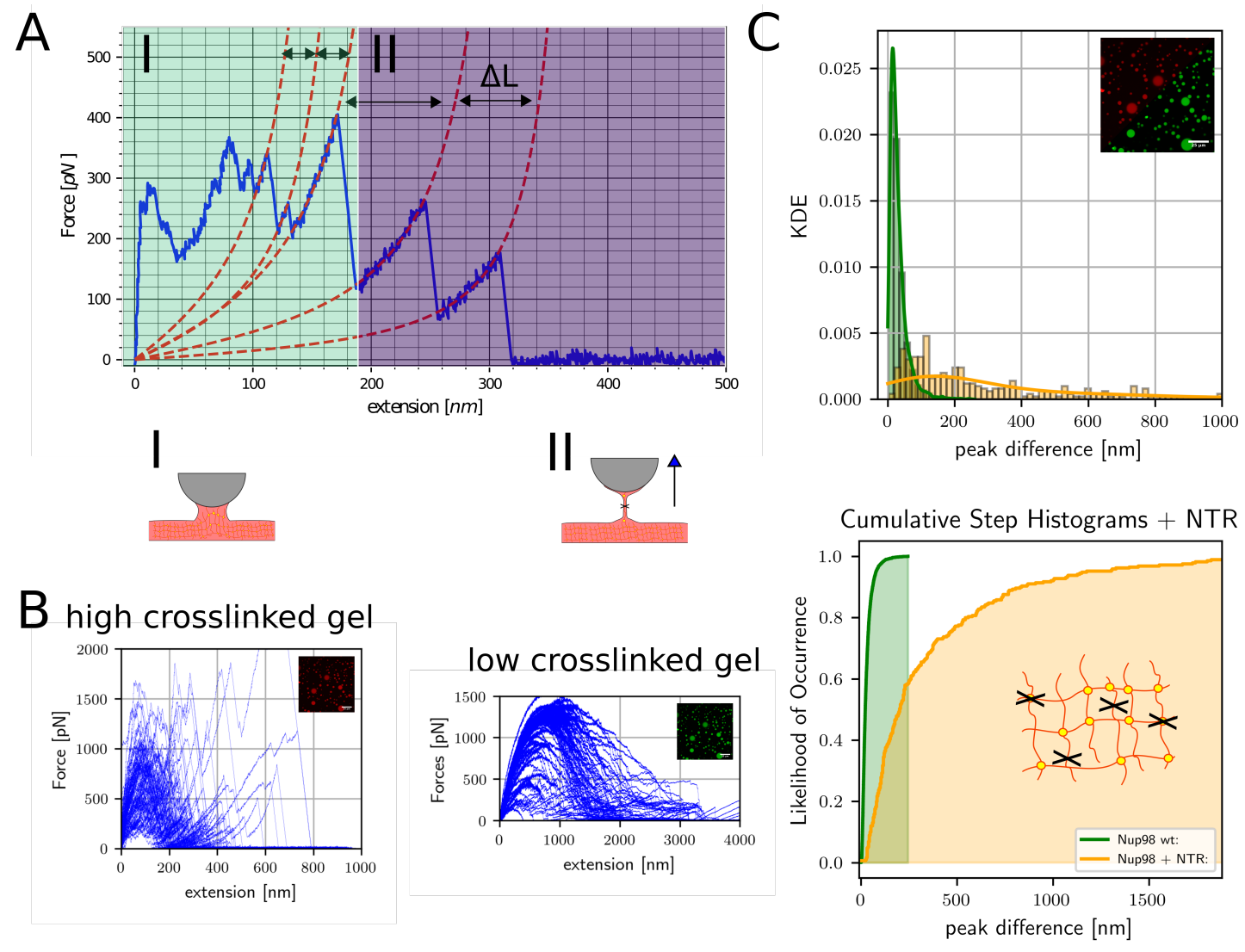

Figure 4.8: Influence of NTR on bulk and single filament rupture events obtained from AFM retraction measurements. A: Representative Force-Extension curve showing sacrificial bond rupture events (I) down to rupture events of single filaments (II) as pull off apart from the underlying FG-Hydrogel matrix. B: Superposition of 1000 ForceExtension curves in the absence (left) and in the presence (right) of NTF2. Comparison of FE-curves reveal softer mechanical responce and a reduction of intermolecular bonds in the presence of NTF2, which is referred to as a low crosslinked gel. C: Histograms and cumulative distributions showing the distribution of distances between single peaks below the contour lenght of Nup98 in the absence of NTR in green and in the presence of NTR in orange.

volume spectroscopy has previously shown that single Nup158 filaments don't show sacrificial bonds and hidden length characteristics from intra-FG-FG-interactions [42]. However, we focus on bulk mechanics, which clearly show hidden-length and we assume that FG-interactions can occur to give rise to a hydrophobic meshwork. This is a region in the force-position histogram below $200 \mathrm{~nm}$ extension as shown in the green marking in Figure $4.9 \mathrm{~B}$ and C. Because we are interested in the influence of NTR on the mechanics and the structure of the hydrogel network, we draw the distribution of position differences in the presence and the absence of NTF2 from this region. Further, we compare the superimposition of force-distance curves as shown in Figure $4.8 \mathrm{~B}$. In the presence of the transporter, the gel changes its mechanical behavior on the single- 
molecule and the bulk level. We believe that it reduces the number of cross-linked interactions and presents a low cross-link gel when NTF2 is incorporated. We make the observation that one can extend strands of the gel up to four times longer than in the absence of NTF2. In addition, the appearance of most of the characteristic bonds vanishes. In the absence of the transporter, therefore, we believe it is a highly crosslinked gel. To estimate the corresponding mesh size, we look at the peak differences between individual rupture events below an extension of $200 \mathrm{~nm}$ as shown in Figure $4.8 \mathrm{C}$ and Figure 4.9 C+D. The maximum of this distribution is $14.2 \mathrm{~nm}$. Therefore, the demolition events between the individual network structures offer a similar order of magnitude to the pore sizes predicted by the poroelastic model. 

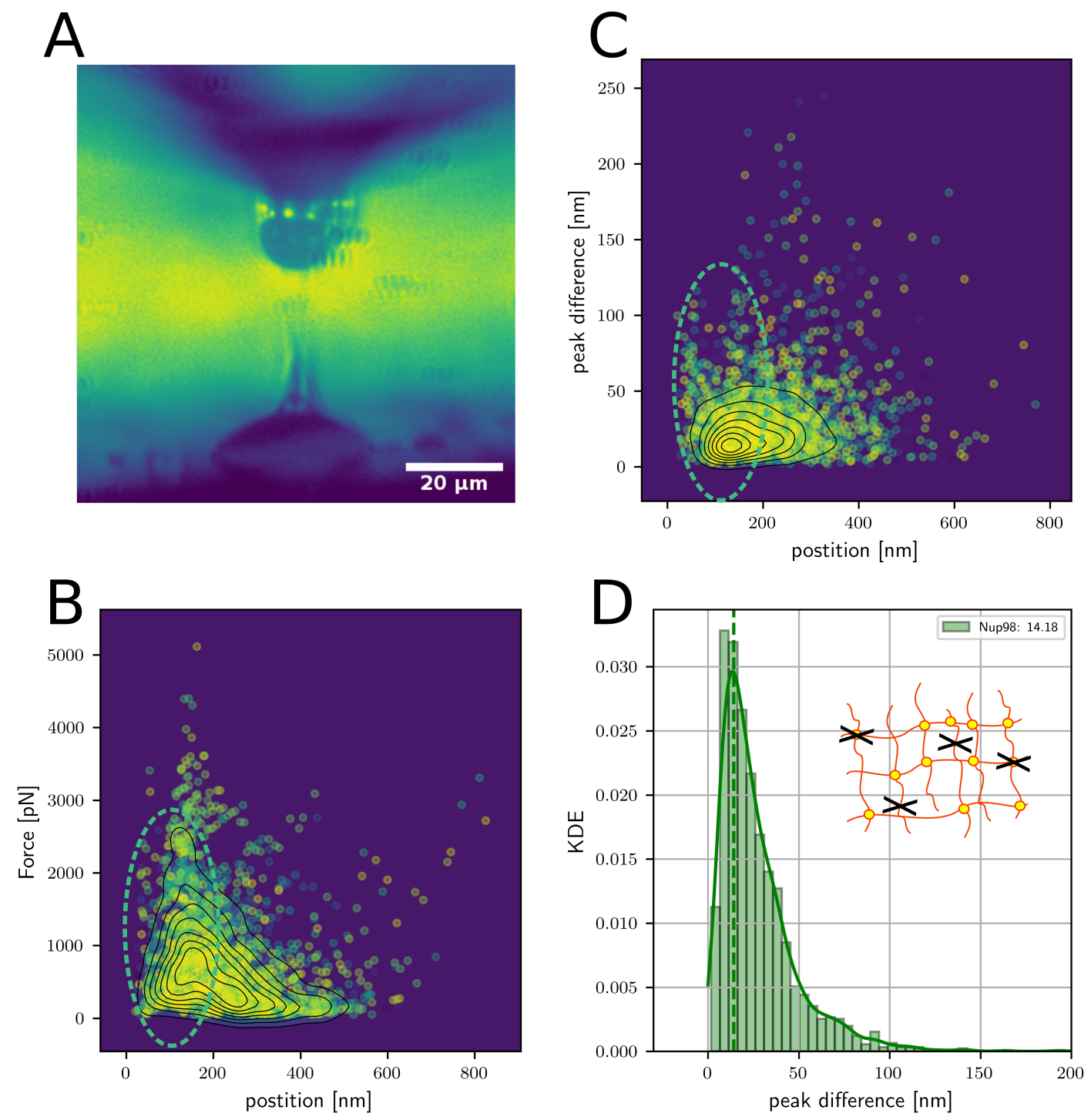

Figure 4.9: Determination of mesh size from bulk rupture events and discrimination from single filament rupture events obtained from AFM retraction measurements. A: AFM-Side-View experiment during retraction phase of AFM cantilever from the FGHydrogel surface. Catenoid bulk is clearly visible between spherical tip and sample surface. Upon further retraction catenoid is thinning towards single filaments. B: Contour plot showing rupture forces as a function of the event from the surface (position). Shown is Force-position-map of interactions between the hydrogel and tip surface at a retraction velocity of $1 \mu \mathrm{m} \mathrm{s}^{-1}$. C: Contour plot showing differences between rupture peaks from B. D: Histogram showing the distribution of distances between single peaks below a tip sample separation of $200 \mathrm{~nm}$. 


\subsection{Conclusion}

In this chapter, we performed the mechanical characterization of phase-separated protein aggregates with Colloidal Probe Force Spectroscopy. This has helped us gather more information about the structure of nucleoporin 98 aggregates, which can be found in the central channel of nuclear pores. The microgel droplets produced from LLPS form a solid model system to investigate nuclear pore transport properties. We also present a method to understand these and other LLPS systems.

In short, it is safe to assume that the FG hydrogel has neither liquid nor completely solid properties. Furthermore, it illustrates a time-dependent inquiring behavior of energy relaxation. The gel is in a condition that is neither entirely liquid nor completely solid. These half liquid-like and half solid-like properties cause several interesting behaviors of relaxation that are not present in either a pure solid or a pure liquid material. We have uncovered an area in which it seems certain that poroelastic behavior is the case. Typically, time-dependent relaxation processes are strongly linked to viscoelastic behavior. Therefore, it is necessary to separate viscoelastic behavior from poroelastic behavior for complete characterization. From AFM microrheology experiments, it is known that these can be clearly separated from each other. Since both relaxation processes can be interlinked depending on frequency, it is crucial to find an experimental area and characterize their proportions [39, 50, 64]. Hu et al. demonstrated how to decipher both components and their ratios with a unified solution for poroelastic oscillation indentation on gels for spherical, conical, and cylindrical indenters. [39].

Assuming pure poroelastic behavior, we have shown that the hydrogel's pore size is in the order of 2 to $15 \mathrm{~nm}$. Considering a formed hydrogel, this will depend on the swelling ratio. Therefore, different osmotic conditions can cause the assemblies to change their structure, mechanical properties, or aging processes of the gel [35]. We have also shown that the mechanical properties change in a transporter's presence and soften the sample. There may even be a possibility that this increases the proportion of viscoelastic behavior. To clarify this, further Rheology experiments would have to be carried out. Of course, under this framework, other mutations, other nuclear pores, and other transporters are of interest. The Poroelastic concept studied with Colloidal Probes is suitable for explaining a liquid and solid insemision of the FG-droplets and combining the sieve-likes exclusion of small molecules below $30 \mathrm{kDa}$ with a water-filled hydrogel. 


\section{Supporting Figures:}

A

Cumulative Step Histograms: Ctr
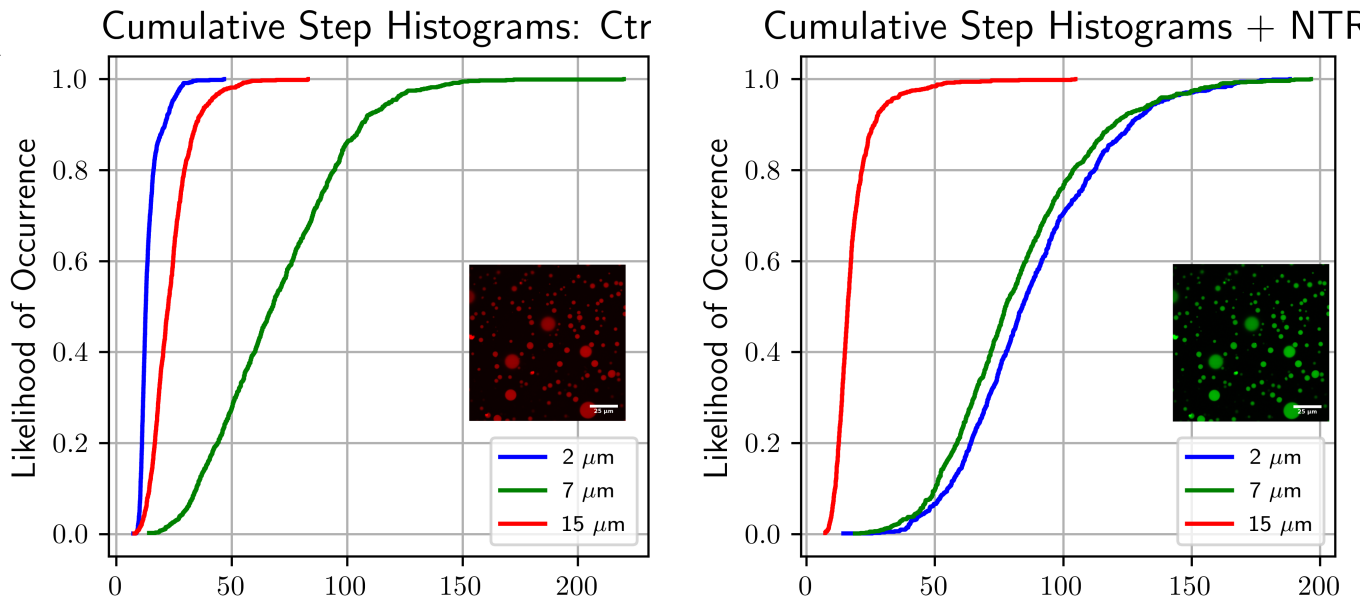

Poroelastic Diffusion Coeffizient $/ \mu \mathrm{m}^{2} \cdot s^{-1}$

Poroelastic Diffusion Coeffizient $/ \mu \mathrm{m}^{2} \cdot s^{-1}$

B

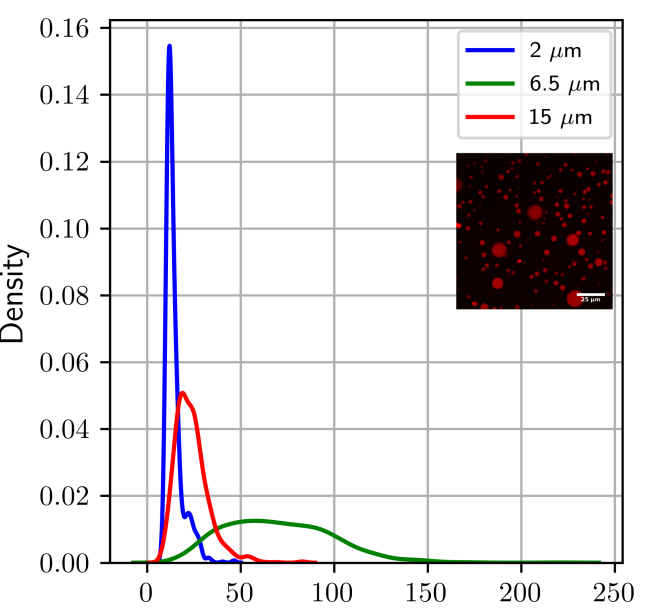

Poroelastic Diffusion Coeffizient $/ \mu \mathrm{m}^{2} \cdot s^{-1}$

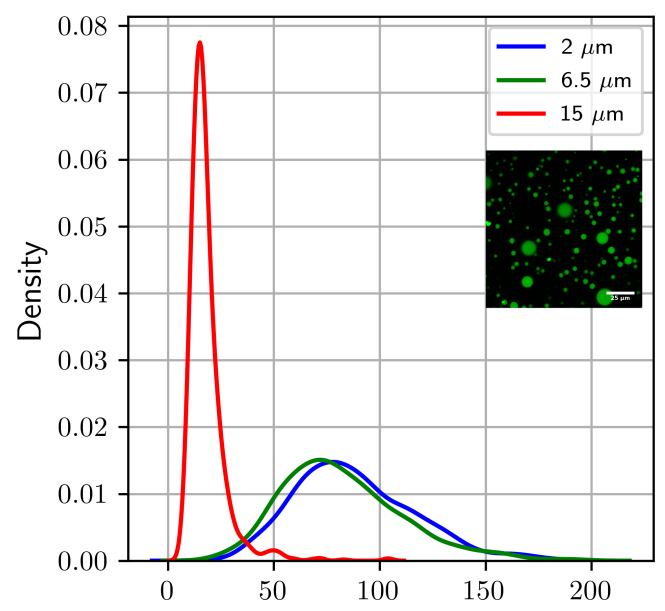

Poroelastic Diffusion Coeffizient $/ \mu \mathrm{m}^{2} \cdot s^{-1}$

Figure 4.10: S1. AFM-based poro-elasticity measurements and the influence of NTR (NTF2) carried out on FG-Hydrogel droplets with a mean diameter of $50 \mu \mathrm{m}$ under variation of AFM-tip size: 2 blue, 6.5 green, and $15 \mu \mathrm{m}$ red diameter. A: Cumulative histograms showing the distribution of pore diffusion coefficients characterizing the re-distributing water content of the FG-Hydrogel after indentation in the absence (left) and in the presence (right) of NTF2. B: Kernel density estimated distributions of A. Experiment performed in Assay buffer (50 mM Tris, $150 \mathrm{mM} \mathrm{NaCl}, \mathrm{pH}=7.5$ ). 

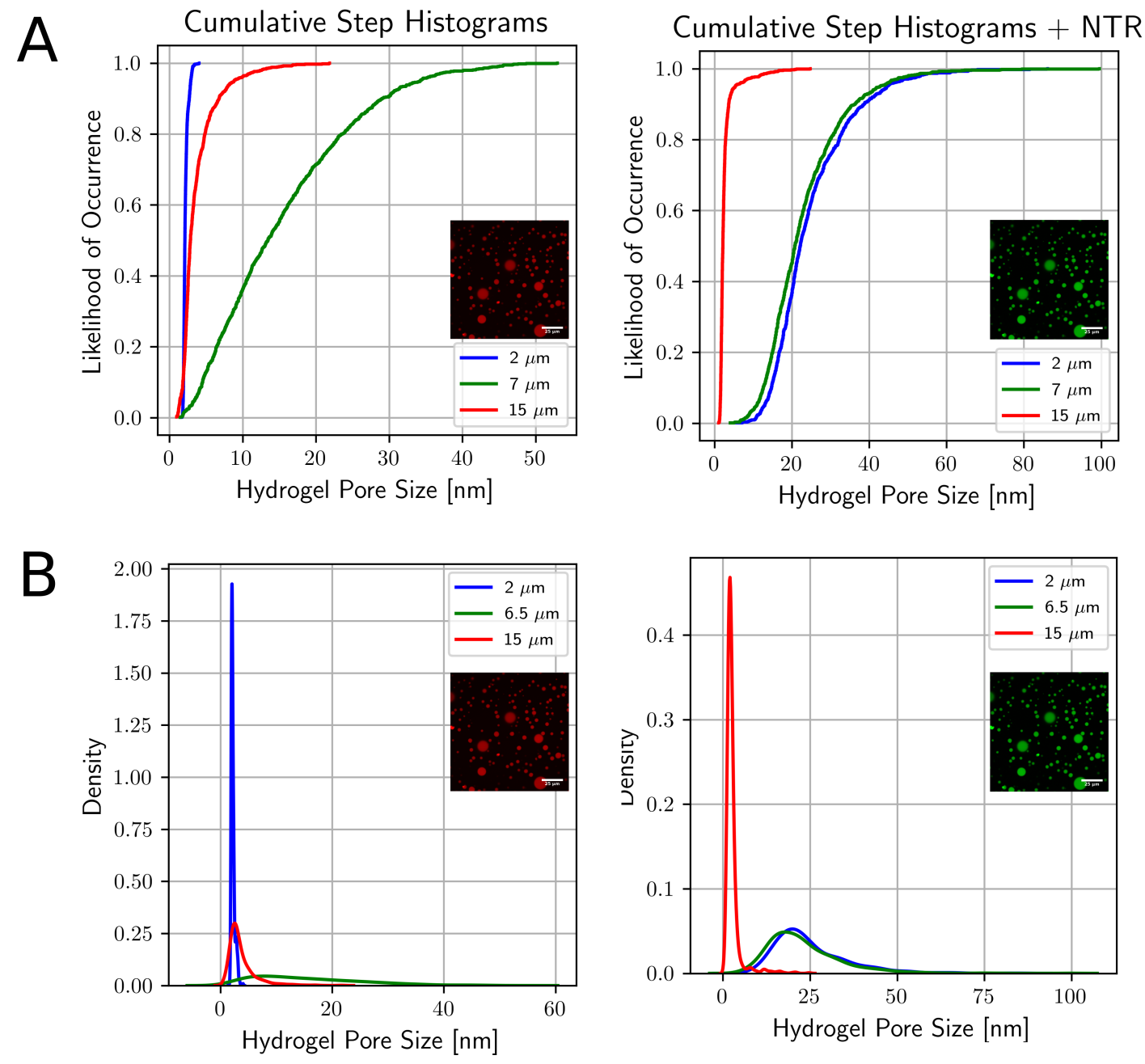

Figure 4.11: S2. AFM-based poro-elasticity measurements and the influence of NTR (NTF2) carried out on FG-Hydrogel droplets with a mean diameter of $50 \mu \mathrm{m}$ under variation of AFM-tip size: 2 blue, 6.5 green, and $15 \mu \mathrm{m}$ red diameter. A: Cumulative histograms showing the distribution of pore size of the FG-Hydrogel after indentation in the absence (left) and in the presence (right) of NTF2. B: Kernel density estimated distributions of A. Experiment performed in Assay buffer ( $50 \mathrm{mM}$ Tris, $150 \mathrm{mM} \mathrm{NaCl}$, $\mathrm{pH}=7.5)$. 
A
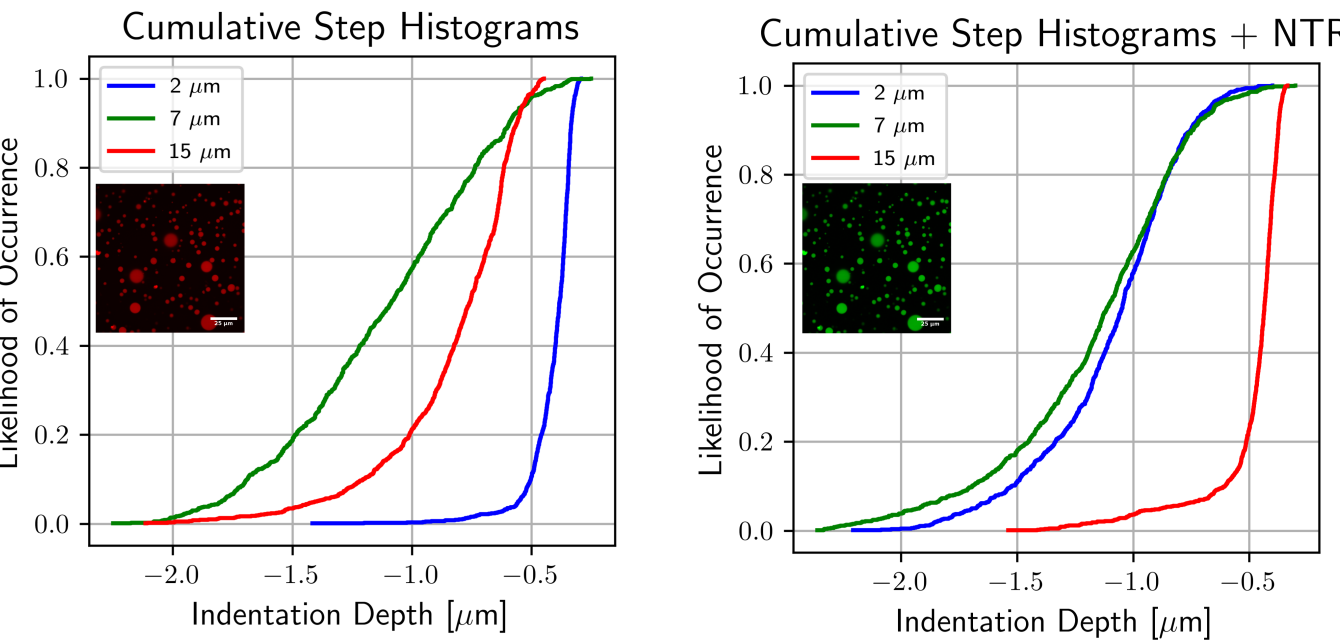

B
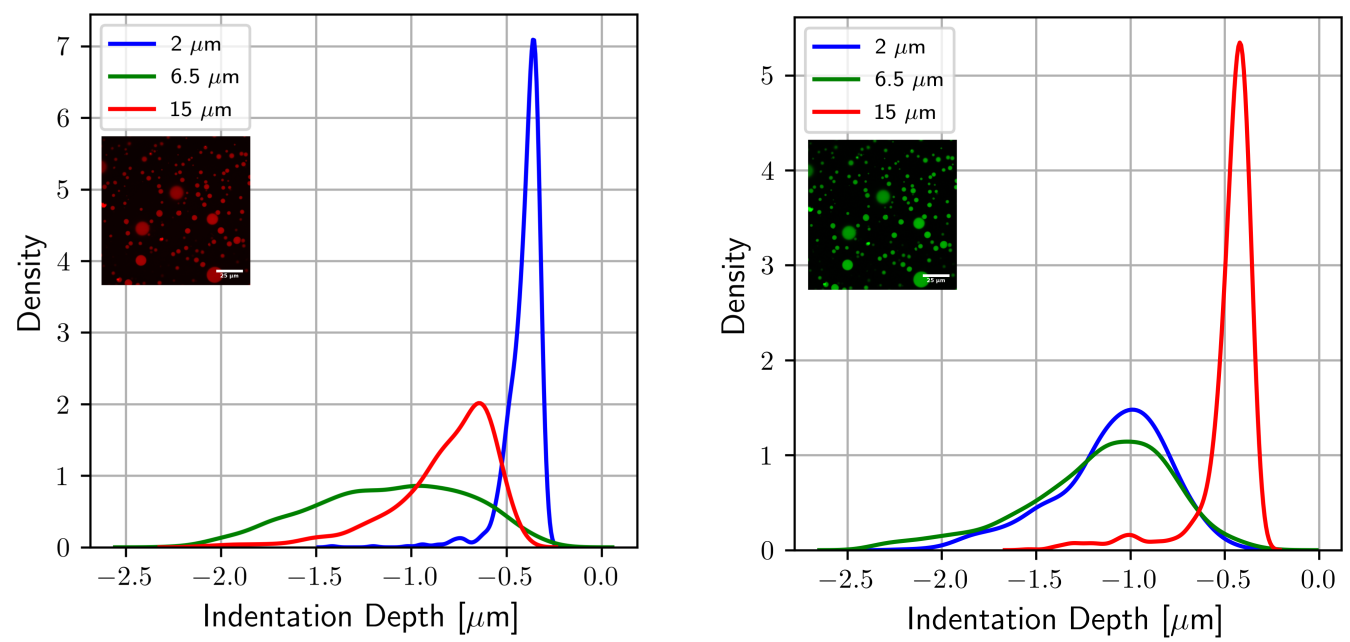

Figure 4.12: S3. AFM-based poro-elasticity measurements and the influence of NTR (NTF2) carried out on FG-Hydrogel droplets with a mean diameter of $50 \mu \mathrm{m}$ under variation of AFM-tip size: 2 blue, 6.5 green, and $15 \mu \mathrm{m}$ red diameter. A: Cumulative histograms showing the distribution of indentation depth of the FG-Hydrogel indentation in the absence (left) and in the presence (right) of NTF2. B: Kernel density estimated distributions of A. Experiment performed in Assay buffer $(50 \mathrm{mM}$ Tris, $150 \mathrm{mM} \mathrm{NaCl}$, $\mathrm{pH}=7.5)$. 

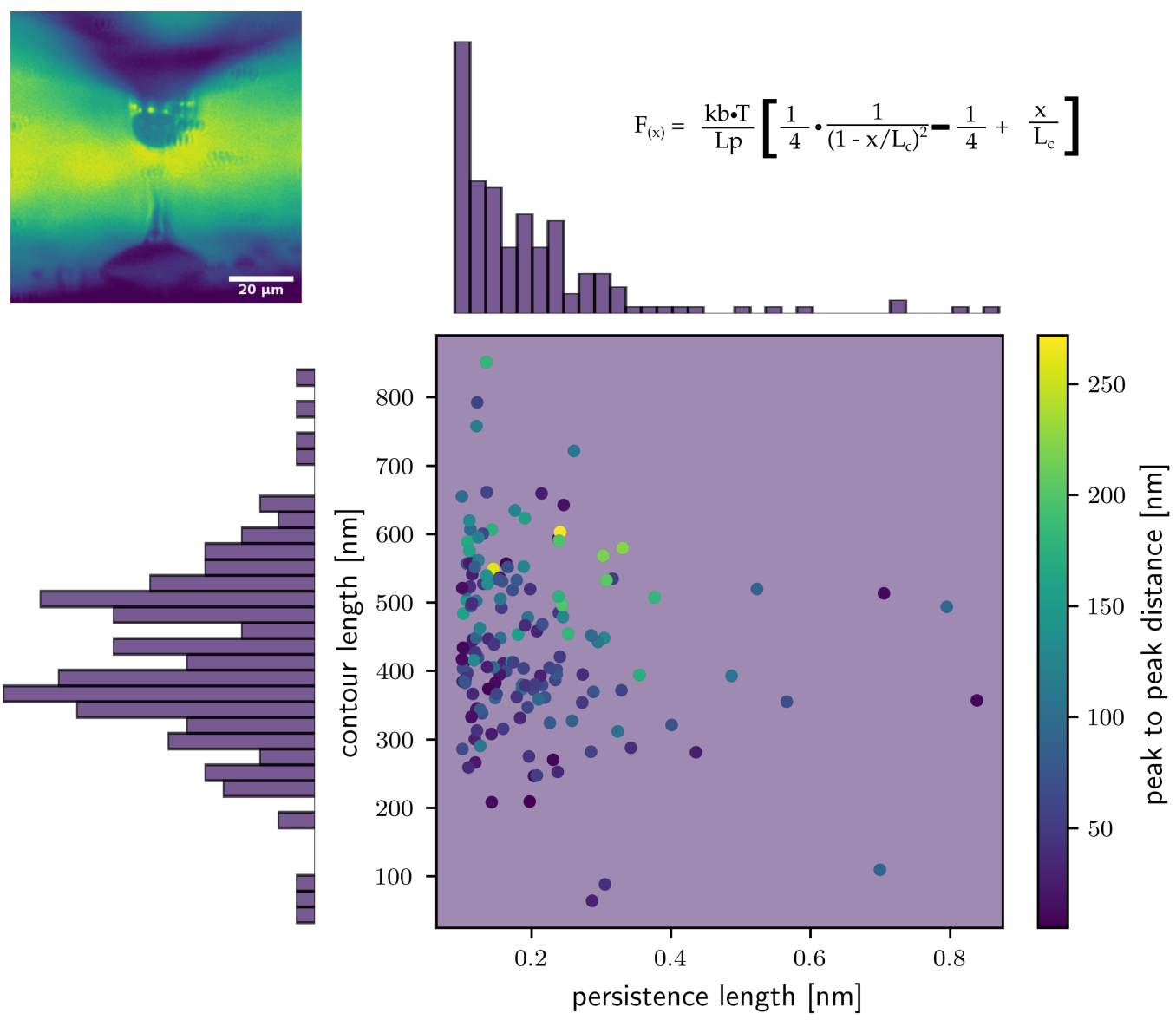

Figure 4.13: S4. Worm-like chain (WLC) analysis of Nup98 SMFS data from force extension of presumptive single filaments. Shown is a scatterplot of contour vs. persistence length obtained from fitting WLC model. Difference between peak-to-peak contour length is shown as color code. 


\section{Reference}

[1] Christian Ader, Steffen Frey, Werner Maas, Hermann Broder Schmidt, Dirk Görlich, and Marc Baldus. Amyloid-like interactions within nucleoporin FG hydrogels. PNAS, 2010. 107. doi:10.1073/pnas.0910163107.

[2] Simon Alberti, Amy Gladfelter, and Tanja Mittag. Considerations and Challenges in Studying Liquid-Liquid Phase Separation and Biomolecular Condensates. Cell, 2019. 176(3): 419-434. doi:10.1016/j.cell.2018.12.035.

[3] Helene Andersen, Ladan Parhamifar, A. Christy Hunter, Victor Shahin, and S. Moein Moghimi. AFM visualization of sub-50 nm polyplex disposition to the nuclear pore complex without compromising the integrity of the nuclear envelope. Journal of Controlled Release, 2016. 244: 24-29. doi:10.1016/j.jconrel.2016.11.008.

[4] Elizabeth E. Antoine, Pavlos P. Vlachos, and Marissa Nichole Rylander. Review of collagen i hydrogels for bioengineered tissue microenvironments: Characterization of mechanics, structure, and transport. Tissue Engineering - Part B: Reviews, 2014. 20(6): 683-696. doi:10.1089/ten.teb.2014.0086.

[5] Iker Valle Aramburu and Edward A. Lemke. Floppy but not sloppy: Interaction mechanism of FG-nucleoporins and nuclear transport receptors. Seminars in Cell and Developmental Biology, 2017. 68: 34-41. doi:10.1016/j.semcdb.2017.06.026.

[6] Anders Aufderhorst-Roberts, Daniel Baker, Richard J. Foster, Olivier Cayre, Johan Mattsson, and Simon D. Connell. Nanoscale mechanics of microgel particles. Nanoscale, 2018. 10(34): 16050-16061. doi:10.1039/c8nr02911c.

[7] W. Michael Babinchak, Raza Haider, Benjamin K. Dumm, Prottusha Sarkar, Krystyna Surewicz, Jin Kyu Choi, and Witold K. Surewicz. The role of liquid-liquid phase separation in aggregation of the TDP-43 low-complexity domain. Journal of Biological Chemistry, 2019. 294(16): 6306-6317. doi:10.1074/jbc.RA118.007222.

[8] Richard Bayliss, Katharina Ribbeck, Debra Akin, Helen M. Kent, Carl M. Feldherr, Dirk Görlich, and Murray Stewart. Interaction between ntf2 and xfxfg-containing nucleoporins is required to mediate nuclear import of rangdp. Journal of Molecular Biology, 1999. 293 3: 579-93.

[9] Joseph D. Berry, Matthew Biviano, and Raymond R. Dagastine. Poroelastic properties of hydrogel microparticles. Soft Matter, 2020. 16(22): 5314-5324. doi: $10.1039 / \mathrm{d} 0 \mathrm{sm} 00191 \mathrm{k}$. 
[10] D. Caccavo, S. Cascone, G. Lamberti, and A. A. Barba. Hydrogels: Experimental characterization and mathematical modelling of their mechanical and diffusive behaviour. Chemical Society Reviews, 2018. 47(7): 2357-2373. doi:10.1039/c7cs00638a.

[11] Sengqiang Cai, Yuhang Hu, Xuanhe Zhao, and Zhigang Suo. Poroelasticity of a covalently crosslinked alginate hydrogel under compression. Journal of Applied Physics, 2010. 108(11). doi:10.1063/1.3517146.

[12] Giorgia Celetti, Giulia Paci, Joana Caria, Virginia VanDelinder, George Bachand, and Edward A. Lemke. The liquid state of FG-nucleoporins mimics permeability barrier properties of nuclear pore complexes. Journal of Cell Biology, 2020. 219(1). doi:10.1083/jcb.201907157.

[13] Shanmei Cheng, Murat Cetinkaya, and Frauke Gräter. How sequence determines elasticity of disordered proteins. Biophysical Journal, 2010. 99(12): 3863-3869. doi:10.1016/j.bpj.2010.10.011.

[14] Charles N Cole and Christopher M Hammell. Nucleocytoplasmic transport: Driving and directing transport Nucleocytoplasmic transport involves assembly and movement across the nuclear envelope of cargo-receptor complexes that interact with the small GTPase Ran. The asymmetric distribution of Ran reg. Current Biology, 1998. 8: 368-372.

[15] Lau Corin. Models of transport through the nuclear pore. Nature Education, 2015. 8: 4-7. doi:10.1038/nature10318.

[16] Santiago O. Correa, Xiaolong Luo, and Christopher B. Raub. Microfluidic fabrication of stable collagen microgels with aligned microstructure using flow-driven co-deposition and ionic gelation. Journal of Micromechanics and Microengineering, 2020. 30(8). doi:10.1088/1361-6439/ab8ebf.

[17] Sujit S. Datta, Ho Cheung Shum, and David A. Weitz. Controlled buckling and crumpling of nanoparticle-coated droplets. Langmuir, 2010. 26(24): 18612-18616. doi:10.1021/la103874z.

[18] Jan Domke and Manfred Radmacher. Measuring the elastic properties of thin polymer films with the atomic force microscope. Langmuir, 1998. 14(12): 3320-3325. doi:10.1021/la9713006.

[19] Dorothee Dormann. FG-nucleoporins caught in the act of liquid-liquid phase separation. The Journal of cell biology, 2020. 219(1): 10-11. doi:10.1083/jcb.201910211. 
[20] O. K. Dudko, G. Hummer, and A. Szabo. Theory, analysis, and interpretation of single-molecule force spectroscopy experiments. Proc Natl Acad Sci, 2008. 105(41): 15755-15760. doi:10.1073/pnas.0806085105.

[21] Ahmed E. Elbanna and Jean M. Carlson. Dynamics of Polymer Molecules with Sacrificial Bond and Hidden Length Systems: Towards a Physically-Based Mesoscopic Constitutive Law. PLoS One, 2013. 8(4). doi:10.1371/journal.pone.0056118.

[22] Shana Elbaum-Garfinkle, Younghoon Kim, Krzysztof Szczepaniak, Carlos ChihHsiung Chen, Christian R. Eckmann, Sua Myong, and Clifford P. Brangwynne. The disordered $\mathrm{P}$ granule protein LAF-1 drives phase separation into droplets with tunable viscosity and dynamics. Proceedings of the National Academy of Sciences, 2015. 112(23): 7189-7194. doi:10.1073/pnas.1504822112.

[23] Mohammad Hadi Esteki, Ali Akbar Alemrajabi, Chloe M. Hall, Graham K. Sheridan, Mojtaba Azadi, and Emad Moeendarbary. A new framework for characterization of poroelastic materials using indentation. Acta Biomaterialia, 2020. 102: 138-148. doi:10.1016/j.actbio.2019.11.010.

[24] Jie Fang, Alexander Mehlich, Nobuyasu Koga, Jiqing Huang, Rie Koga, Xiaoye Gao, Chunguang Hu, Chi Jin, Matthias Rief, Juergen Kast, David Baker, and Hongbin Li. Forced protein unfolding leads to highly elastic and tough protein hydrogels. Nature Communications, 2013. 4. doi:10.1038/ncomms3974.

[25] Georg E. Fantner, Tue Hassenkam, Johannes H. Kindt, James C. Weaver, Henrik Birkedal, Leonid Pechenik, Jacqueline A. Cutroni, Geraldo A.G. Cidade, Galen D. Stucky, Daniel E. Morse, and Paul K. Hansma. Sacrificial bonds and hidden length dissipate energy as mineralized fibrils separate during bone fracture. Nature Materials, 2005. 4(8): 612-616. doi:10.1038/nmat1428.

[26] Zhe Feng, Menglong Zeng, Xudong Chen, and Mingjie Zhang. Neuronal Synapses: Microscale Signal Processing Machineries Formed by Phase Separation? Special Issue: Membrane-Less Organelles. Biochemistry, 2018. 57(17): 2530-2539. doi: 10.1021/acs.biochem.8b00313.

[27] Ben Fogelson and James P. Keener. Enhanced Nucleocytoplasmic Transport due to Competition for Elastic Binding Sites. Biophysical Journal, 2018. 115(1): 108-116. doi:10.1016/J.BPJ.2018.05.034.

[28] Steffen Frey and Dirk Görlich. A Saturated FG-Repeat Hydrogel Can Reproduce 
the Permeability Properties of Nuclear Pore Complexes. Cell, 2007. 130(3): 512-523. doi:10.1016/j.cell.2007.06.024.

[29] Steffen Frey, Ralf P Richter, and Dirk Görlich. FG-rich repeats of nuclear pore proteins form a three-dimensional meshwork with hydrogel-like properties. Science (New York, NY), 2006. 314(5800): 815-7. doi:10.1126/science.1132516.

[30] E. R. Griffis. Nup98 Is a Mobile Nucleoporin with Transcription-dependent Dynamics. Molecular Biology of the Cell, 2002. doi:10.1091/mbc.01-11-0538.

[31] Thomas Heida, Jens W. Neubauer, Maximilian Seuss, Nicolas Hauck, Julian Thiele, and Andreas Fery. Mechanically Defined Microgels by Droplet Microfluidics. Macromolecular Chemistry and Physics, 2017. 218(2): 1-19. doi:10.1002/macp.201600418.

[32] Bastian B. Hülsmann, Aksana A. Labokha, and Dirk Görlich. The permeability of reconstituted nuclear pores provides direct evidence for the selective phase model. Cell, 2012. 150(4): 738-751. doi:10.1016/j.cell.2012.07.019.

[33] Anthony A. Hyman, Christoph A. Weber, and Frank Jülicher. Liquid-Liquid Phase Separation in Biology. Annual Review of Cell and Developmental Biology, 2014. 30(1): 39-58. doi:10.1146/annurev-cellbio-100913-013325.

[34] Arkaitz Ibarra and Martin W. Hetzer. Nuclear pore proteins and the control of genome functions. Genes and Development, 2015. 29(4): 337-349. doi:10.1101/gad. 256495.114.

[35] Louise Jawerth, Elisabeth Fischer-friedrich, Suropriya Saha, Jie Wang, Mahdiye Ijavi, Shambaditya Saha, Anthony A Hyman, and J Frank. Protein condensates as aging Maxwell fluids. Science (New York, NY), 2019. 1323(December): 1-21.

[36] Larisa E. Kapinos, Rafael L. Schoch, Raphael S. Wagner, Kai D. Schleicher, and Roderick Y.H. Lim. Karyopherin-centric control of nuclear pores based on molecular occupancy and kinetic analysis of multivalent binding with FG nucleoporins. Biophysical Journal, 2014. 106(8): 1751-1762. doi:10.1016/j.bpj.2014.02.021.

[37] Tatyana G Kuznetsova, Maria N Starodubtseva, Nicolai I Yegorenkov, Sergey A Chizhik, and Renat I Zhdanov. Atomic force microscopy probing of cell elasticity. Micron, 2007. 38(8): 824-833. doi:10.1016/j.micron.2007.06.011.

[38] Aksana A. Labokha, Sabine Gradmann, Steffen Frey, Bastian B. Hülsmann, Henning Urlaub, Marc Baldus, and Dirk Görlich. Systematic analysis of barrier- 
forming FG hydrogels from Xenopus nuclear pore complexes. EMBO Journal, 2013. 32(2): 204-218. doi:10.1038/emboj.2012.302.

[39] Yang Lai and Yuhang Hu. Unified solution for poroelastic oscillation indentation on gels for spherical, conical and cylindrical indenters. Soft Matter, 2017. 13(4): 852-861. doi:10.1039/c6sm02341j.

[40] Edward A. Lemke. The Multiple Faces of Disordered Nucleoporins. Journal of Molecular Biology, 2016. 428(10): 2011-2024. doi:10.1016/j.jmb.2016.01.002.

[41] Arnaud Ruppert David levine susan hanson maureen Germain. Studying Protein Aggregation in the Context of Liquid. Physiology \& behavior, 2017. 176(3): 139-148. doi:10.21769/bioprotoc.3489.Studying.

[42] Roderick Y.H. Lim, Joachim Köser, Ning ping Huang, Kyrill Schwarz-Herion, and Ueli Aebi. Nanomechanical interactions of phenylalanine-glycine nucleoporins studied by single molecule force-volume spectroscopy. Journal of Structural Biology, 2007. 159(2 SPEC. ISS.): 277-289. doi:10.1016/j.jsb.2007.01.018.

[43] A. Loschberger, S. van de Linde, M.-C. Dabauvalle, B. Rieger, M. Heilemann, G. Krohne, and M. Sauer. Super-resolution imaging visualizes the eightfold symmetry of gp210 proteins around the nuclear pore complex and resolves the central channel with nanometer resolution. Journal of Cell Science, 2012. doi: $10.1242 /$ jcs.098822.

[44] Andrea Malandrino and Emad Moeendarbary. Poroelasticity of Living Tissues. Elsevier Inc., 2017. ISBN 9780128012383. doi:10.1016/b978-0-12-801238-3.99932-x.

[45] Emad Moeendarbary, Léo Valon, Marco Fritzsche, Andrew R. Harris, Dale A. Moulding, Adrian J. Thrasher, Eleanor Stride, L. Mahadevan, and Guillaume T. Charras. The cytoplasm of living cells behaves as a poroelastic material. Nature Materials, 2013. 12(3): 253-261. doi:10.1038/nmat3517.

[46] Pezhman Mohammadi, A. Sesilja Aranko, Laura Lemetti, Zoran Cenev, Quan Zhou, Salla Virtanen, Christopher P. Landowski, Merja Penttilä, Wolfgang J. Fischer, Wolfgang Wagermaier, and Markus B. Linder. Phase transitions as intermediate steps in the formation of molecularly engineered protein fibers. Communications Biology, 2018. 1(1). doi:10.1038/s42003-018-0090-y.

[47] Bracha Naim, David Zbaida, Shlomi Dagan, Ruti Kapon, and Ziv Reich. Cargo sur- 
face hydrophobicity is suf cient to overcome the nuclear pore complex selectivity barrier. Molecular Biology, 2009: 1-9. doi:10.1038/emboj.2009.225.

[48] KC Keir C Neuman and Attila Nagy. Single-molecule force spectroscopy: optical tweezers, magnetic tweezers and atomic force microscopy. Nature methods, 2008. 5(6): 491-505. doi:10.1038/nmeth.1218.Single-molecule.

[49] Victor N Nikolaevskiy. Theory and Applications of Transport in Porous Media (Book 8). 1995. ISBN 978-0792337935.

[50] Ramin Oftadeh, Brianne K. Connizzo, Hadi Tavakoli Nia, Christine Ortiz, and Alan J. Grodzinsky. Biological connective tissues exhibit viscoelastic and poroelastic behavior at different frequency regimes: Application to tendon and skin biophysics. Acta Biomaterialia, 2018. 70: 249-259. doi:10.1016/j.actbio.2018.01.041.

[51] Evgeny Onischenko, Jeffrey H. Tang, Kasper R. Andersen, Kevin E. Knockenhauer, Pascal Vallotton, Carina P. Derrer, Annemarie Kralt, Christopher F. Mugler, Leon Y. Chan, Thomas U. Schwartz, and Karsten Weis. Natively Unfolded FG Repeats Stabilize the Structure of the Nuclear Pore Complex. Cell, 2017. 171(4): 904-917.e19. doi:10.1016/j.cell.2017.09.033.

[52] M. L. Oyen. Mechanical characterisation of hydrogel materials. International Materials Reviews, 2014. 59(1): 44-59. doi:10.1179/1743280413Y.0000000022.

[53] Michelle L Oyen. Poroelastic nanoindentation responses of hydrated bone. Journal of Materials Research, 2018. 23(5): 1307-1314. doi:10.1557/JMR.2008.0156.

[54] Avinash Patel, Hyun O. Lee, Louise Jawerth, Shovamayee Maharana, Marcus Jahnel, Marco Y. Hein, Stoyno Stoynov, Julia Mahamid, Shambaditya Saha, Titus M. Franzmann, Andrej Pozniakovski, Ina Poser, Nicola Maghelli, Loic A. Royer, Martin Weigert, Eugene W. Myers, Stephan Grill, David Drechsel, Anthony A. Hyman, and Simon Alberti. A Liquid-to-Solid Phase Transition of the ALS Protein FUS Accelerated by Disease Mutation. Cell, 2015. 162(5): 1066-1077. doi:10.1016/j. cell.2015.07.047.

[55] Reiner Peters. Functionalization of a nanopore: The nuclear pore complex paradigm, 2009. doi:10.1016/j.bbamcr.2009.06.003.

[56] Elias M. Puchner, Gereon Franzen, Mathias Gautel, and Hermann E. Gaub. Comparing proteins by their unfolding pattern. Biophysical Journal, 2008. 95(1): 426-434. doi:10.1529/biophysj.108.129999. 
[57] Shruti Rattan and Alfred J. Crosby. Effect of Polymer Volume Fraction on Fracture Initiation in Soft Gels at Small Length Scales. ACS Macro Letters, 2019: 492-498. doi:10.1021/acsmacrolett.9b00086.

[58] Veronique Receveur-Brechot and Dominique Durand. How random are intrinsically disordered proteins? A small angle scattering perspective. Current protein $\mathcal{E}$ peptide science, 2012. 13(1): 55-75. doi:10.2174/138920312799277901.

[59] Felix Rico, Annafrancesca Rigato, Laura Picas, and Simon Scheuring. Mechanics of proteins with a focus on atomic force microscopy. J Nanobiotechnology, 2013. 11 Suppl 1(Suppl 1): S3. doi:10.1186/1477-3155-11-S1-S3.

[60] Kai D. Schleicher, Simon L. Dettmer, Larisa E. Kapinos, Stefano Pagliara, Ulrich F. Keyser, Sylvia Jeney, and Roderick Y.H. Lim. Selective transport control on molecular velcro made from intrinsically disordered proteins. Nature Nanotechnology, 2014. 9(7): 525-530. doi:10.1038/nnano.2014.103.

[61] Hermann B.roder Schmidt and Dirk Görlich. Nup98 FG domains from diverse species spontaneously phase-separate into particles with nuclear pore-like permselectivity. eLife, 2015. 4: 1-30. doi:10.7554/eLife.04251.

[62] Yongdae Shin and Clifford P. Brangwynne. Liquid phase condensation in cell physiology and disease. Science, 2017. 357(6357). doi:10.1126/science.aaf4382.

[63] George J. Stanley, Ariberto Fassati, and Bart W. Hoogenboom. Biomechanics of the transport barrier in the nuclear pore complex. Seminars in Cell and Developmental Biology, 2017. 68: 42-51. doi:10.1016/j.semcdb.2017.05.007.

[64] Daniel G T Strange, Timothy L. Fletcher, Khaow Tonsomboon, Helen Brawn, Xuanhe Zhao, and Michelle L. Oyen. Separating poroviscoelastic deformation mechanisms in hydrogels. Applied Physics Letters, 2013. 102(3): 3-7. doi:10.1063/1. 4789368.

[65] Richard C. Tolman. The Effect of Droplet Size on Surface Tension. The Journal of Chemical Physics, 1949. 17(3): 333-337. doi:10.1063/1.1747247\&TITLE=THE+EFFECT+ $\mathrm{OF}+\mathrm{DROPLET}+\mathrm{SIZE}+\mathrm{ON}+\mathrm{SURFACE}+\mathrm{TENSION} \& \mathrm{SUMMARY}=\& S O U R C E=$.

[66] Andrei Vovk, Chad Gu, Michael G. Opferman, Larisa E. Kapinos, Roderick Y.H. Lim, Rob D. Coalson, David Jasnow, and Anton Zilman. Simple biophysics underpins collective conformations of the intrinsically disordered proteins of the nuclear pore complex. eLife, 2016. doi:10.7554/eLife.10785. 
[67] Sarah Wälde and Ralph H. Kehlenbach. The Part and the Whole: Functions of nucleoporins in nucleocytoplasmic transport. Trends in Cell Biology, 2010. 20(8): 461-469. doi:10.1016/j.tcb.2010.05.001.

[68] Emily B. Walton, Sunyoung Lee, and Krystyn J. Van Vliet. Extending Bell's model: How force transducer stiffness alters measured unbinding forces and kinetics of molecular complexes. Biophysical Journal, 2008. 94(7): 2621-2630. doi: 10.1529/biophysj.107.114454.

[69] Raphael Zahn, Dino Osmanović, Severin Ehret, Carolina Araya Callis, Steffen Frey, Murray Stewart, Changjiang You, Dirk Goerlich, Bart W. Hoogenboom, and Ralf P. Richter. A physical model describing the interaction of nuclear transport receptors with FG nucleoporin domain assemblies. eLife, 2016. 5(APRIL2016): 1-21. doi:10.7554/eLife.14119.

[70] Joanna Zemła, Justyna Bobrowska, Andrzej Kubiak, Tomasz Zieliński, Joanna Pabijan, Katarzyna Pogoda, Piotr Bobrowski, and Małgorzata Lekka. Indenting soft samples (hydrogels and cells) with cantilevers possessing various shapes of probing tip. European Biophysics Journal, 2020. 49(6): 485-495. doi:10.1007/ s00249-020-01456-7. 


\section{CHAPTER 5}

\section{CELLULAR MECHANICS DURING MYOGENISIS OF MYOBLAST CELLS}

Cell mechanical properties, during migration, differentiation, reaction to external stimuli, and many diseases' pathogenesis, play an essential role in several cell biological processes. The mechanical properties of cells depend on the cytoskeleton's dynamic organization and its interaction with the extracellular environment. While remarkable research advances have been made in cell mechanics in the last decades, a practical mechanical model of single living cells is still wanted. This project aimed to research and measure the mechanical properties of muscle cells and check their potential changes in cytoskeleton disruption and pathology conditions in the context of fusion efficiency and the influence of membrane tension.

As we studied with AFM nanoindentation technique, we perfomed an experimental approach to decode the typical mechanical behaviors of living $\mathrm{C} 2 \mathrm{C} 12$ myoblasts as precursors muscle cells and myotubes, which are differentiated muscle cells.

The myoblast's mechanical behavior is an active process that depends intensely on the dynamics of the cytoskeleton [8, 17]. Therefore it is crucial bringing this into an artificial model system like colloidal probe force spectroscopy to study membrane fusion and the role of featuring biomolecules like Myomerger and Myomaker. This chapter tried to answer how the Myomerger ectodomain drives fusion pore formation by generating membrane stress.

Membrane fusion is a multi-step process that unites two membranes and their surrounding volumes upon completion [6]. It is necessary for various biological processes, including exocytosis, viral infection, and syncytial tissue growth [21, 22, 27]. By resolving energy barriers through the creation of fusion intermediates, protein fusion machines promote membrane merging. The commonly accepted mechanism for this 
is based on protein interactions anchored in each of the membranes. The proteins undergo a conformational shift that bends and brings the membranes close to each other. Examples of this mechanism involve intracellular vesicle fusion mediated by SNARE, discussed later in this thesis.

Typical mechanisms of membrane fusion involve transmembrane proteins that assemble into complexes and dynamically modify their conformation to bend membranes, resulting in membrane contact followed by mixing membrane lipids and mixing contents through opening a fusion pore [18]. Myomaker and Myomerger are structurally divergent from typical fusogenic proteins but control myoblast fusion and muscle formation [12]. It is shown in the literature that Myomaker and Myomerger independently mediate distinct phases in the fusion pathway [7]. Myomaker is involved in membrane hemifusion, and fusion pore formation involves Myomerger. Mechanistically, on the cell surface where the ectodomain stress membranes are needed, that Myomerger is required [3]. Also, it is shown that Myomerger drives fusion completion in heterologous systems independent of Myomaker and that there is no need for a Myomaker-Myomerger physical interaction for operation [19].

When retained in a growth factor weak medium, C2C12 myoblasts undergo myogenic differentiation, such as in low-serum conditions. Once $80 \%$ cell confluence is reached, C2C12 myoblast myogenic differentiation can be initiated by replacing the growth medium (GM) with a differentiation medium (DM); see Table 3.1.1] [7, 17, 24]. Every 24 hours, from 1 hour before changing the medium until 5 days after differentiation induction, we monitored their differentiation process on a collagen-coated petri dish by phase contrast and fluorescence microscopy. C2C12 myoblasts tended not to be connecting until inducing the differentiation, maintaining their characteristic morphology. Myoblasts were able to complete one full cell cycle 24 hours before substituting GM for DM, while the number of cells had increased. Cells then tended to be confluent, as shown in Figure 5.1 .

Even under these circumstances, some myoblasts can continue to proliferate. This expresses the in vivo self-renewal capability of myoblasts in vitro phenomenon [10]. In particular, some myoblasts undergoing mitosis, and thus are not subject to differentiation, were also detected within 120 hours during the observation period. A more elongated form and a more aligned organization were shown by $\mathrm{C} 2 \mathrm{C} 12$ cells on the second day of differentiation. On the third day, cells were still reshaping and showed isolated smaller cell organizations. Myoblasts fused their cell membranes over the following 24 hours and formed multinucleated elongated cells: the myotubes. Myotubes matured by the fusion of other myoblasts on the fifth day of differentiation, as they 


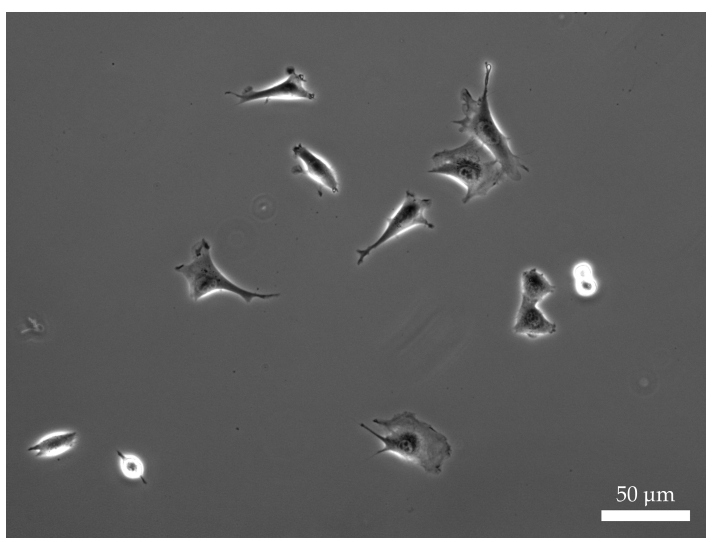

(a) single cells

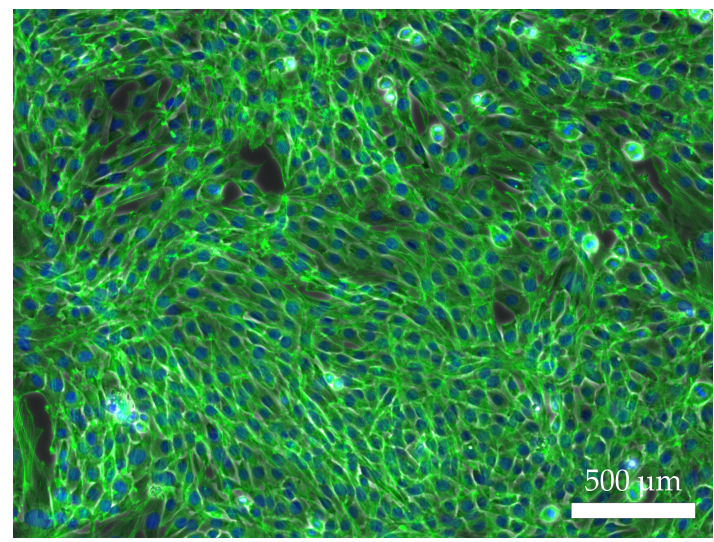

(b) confluent layer

Figure 5.1: Phase contrast and fluorescence images of $\mathrm{C} 2 \mathrm{C} 12$ on collagen-coated petri dishes. A: Phase contrast image of $\mathrm{C} 2 \mathrm{C} 12$ myoblasts adherent on a collagen coated petri dish. B: Fluorescence microscopy images of C2C12 myoblast F-actin microfilaments (green) stained with phalloidin-Alexa Fluor 488 and nuclei (blue) stained with DAPI. A confluent cell layer is shown.

display a higher number of centrally located nuclei. 


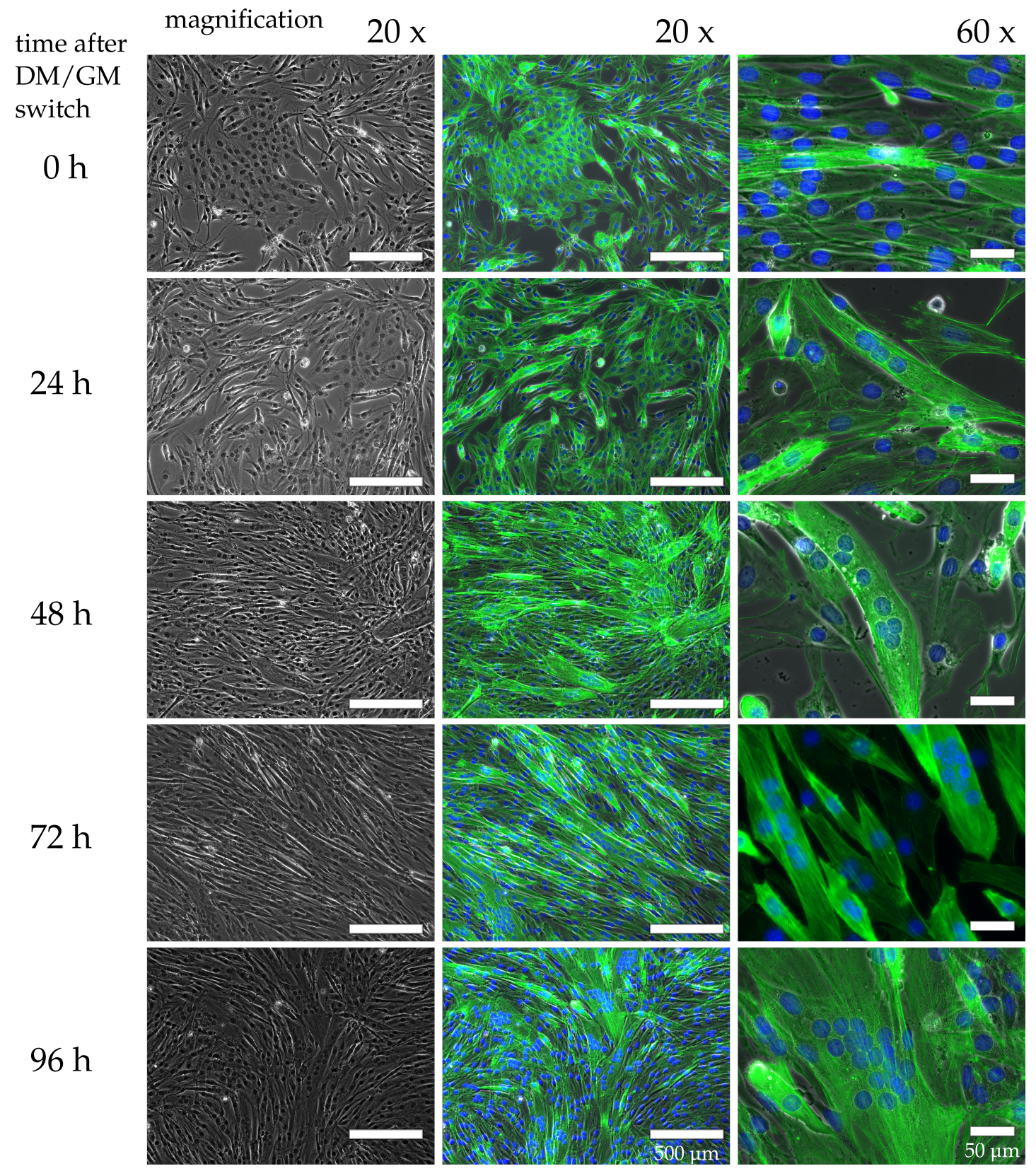

Figure 5.2: $\mathrm{C} 2 \mathrm{C} 12$ myogenic differentiation process. Phase contrast microscopy images captured with the low and large objective magnification. The observations were performed every 24 hours, starting before changing the medium until the fifth day of differentiation. Cells were grown on collagen-coated petri dishes. Fluorescence microscopy images of $\mathrm{C} 2 \mathrm{C} 12$ myoblast F-actin microfilaments (green) stained with phalloidin-Alexa Fluor 488 and nuclei (blue) stained with DAPI. 


\subsection{Atomic Force Microscopy indentation and retraction expe- riments}

One can define the mechanical properties of cells in several ways. A brief introduction is given in this section on how the mechanical activity of single, confluent, and differentiating $\mathrm{C} 2 \mathrm{C} 12$ cells is measured. The dynamics of $\mathrm{C} 2 \mathrm{C} 12$ cells were studied using AFM indentation (Fig. 5.3) followed by indenter retraction (Fig. 5.4). In force-indentation curves, the mechanical response of the indented cell is reflected. Additionally to the Hertzian mechanics, the tension model was applied to measure cells' mechanical properties in the context of cortical tension. Tether rupture forces from the retraction curves were obtained and analyzed to reveal membrane tension changes during the differentiation phase.

Force indentation and tether pulling experiments were performed with a NanoWizard III AFM (JPK Instruments). C-levers from MLCT-Cantilevers (Bruker AFM Probes, Camarillo, CA) with pyramidal indenter geometry were used. In order to facilitate adhesive contact between the cantilever tip and the membrane resulting in tether pulling, the cantilever chips were carefully washed with PBS (Biochrom) and isopropanol and incubated for 90 minutes in $2.5 \mathrm{mg} \mathrm{ml}^{-1}$ FITC-conjugated concanavalin A (SigmaAldrich, Steinheim, Germany) in PBS. The force curves were recorded in force-mapping mode at a tip velocity of $3 \mu \mathrm{m} \mathrm{s}^{-1}$, a set point of $1 \mathrm{nN}$ and a resolution of $2 \mu \mathrm{m} /$ pixel. The dwell time between extension and retraction of the cantilever was chosen to be $0.5 \mathrm{~s}$ in order to increase the probability of membrane adhesion to the cantilever tip and thereby promote tether pulling. In order to calibrate the received signal, the sensitivity and the spring constant of the cantilever were calculated using the thermal noise method [4]. Prior to analysis of the experimental data, the baselines of the force curves were corrected and the contact point determined by visual inspection. Force curves which did not feature a distinct contact point or exhibited a significant drop in force after indentation were excluded from analysis. The contact regimes of the processed curves were fitted with a polynomial function (see equation 2.17) calculating the force (F) from the indentation depth (d), using coefficients depending on the apparent cellular pre-tension $\left(\tilde{T}_{0}\right)$ and the apparent area compressibility modulus $\left(\tilde{K}_{A}\right)$ as fit parameters.

The mechanical properties for $\mathrm{C} 2 \mathrm{C} 12$ cells during different stages of differentiation are summarized in Table 5.1. There has been a comparative need to understand myoblast fusion mechanism and regulation compared to other events that precede it during differentiation. Plasma membrane fusion is a complex process that requires recognition, adhesion, cell signaling, cytoskeletal alterations, and membrane rearrangement [2, 12]. We observe that morphology and intracellular structure change dramatically during 
differentiation, leading to a very different cytoskeletal organization than in myoblasts single cells and, therefore, different mechanical reactions [23]. We observe this in our indentation experiments in that the stiffness of individual cells initially doubles from $12.96 \mathrm{kPa}$ to $25.02 \mathrm{kPa}$ as soon as a confluent cell layer has formed. These cells are considered to be the starting point for cellular fusion in our experiments. Fusion is triggered by switching to DM. At the same time, we observe an increase in cortical tension. As soon as the first cells are fused, and small cell fibers with nuclei less than three are formed, the cells' stiffness and tension decrease. We assume that this mechanical change is mainly caused by the rearrangement of the actin structure and the cytoskeleton after two cells' fusion [11, 13]. We also note that the context or confinement of a living cell has a precise impact on the measured elastic modulus [15].

\begin{tabular}{l|ccc} 
(Mean +- SD) & $E_{\text {Young }}[\mathrm{kPa}]$ & $\tilde{T}_{0}[\mathrm{mN} / \mathrm{m}]$ & $t_{\text {tether }}[\mathrm{mN} / \mathrm{m}]$ \\
\hline single cell & $12.96 \pm 9.15$ & $1.05 \pm 0.57$ & $0.13 \pm 0.07$ \\
confluent layer & $25.02 \pm 13.6$ & $1.82 \pm 0.75$ & $0.11 \pm 0.07$ \\
small fiber & $3.52 \pm 1.40$ & $0.54 \pm 0.22$ & $0.09 \pm 0.07$ \\
mature myotube & $17.3 \pm 1.72$ & $0.74 \pm 0.62$ & $0.06 \pm 0.04$
\end{tabular}

Table 5.1: Mechanical properties of $\mathrm{C} 2 \mathrm{C} 12$ cells from AFM-based indentation and retraction experiments.

During differentiation, the unique event is membrane fusion, which causes a breakdown of the cell membrane and some significant rearrangement of the cytoskeleton. Initially, fusion concerns mononucleated myoblasts; then, myoblast-myotube and myotube-myotube fusions begin to occur. Nowak et al. [13] demonstrated the following specific events that took place before and during myoblast fusion: (i) the evolution of the elongated bipolar form of myoblast fusion, (ii) the alteration of their membrane organization, and (iii) the dynamic modification of lamellipodia and filipodia in the formation of myotubes. The pre-fusing myoblasts have a bipolar elongated shape before they undergo plasma membrane fusion. Such re-organization of cell morphology requires non-muscle myosin II and actin dynamics, cadherins, integrins, and DOCK1. Genetic studies have also shown the vital role of actin in myoblast membrane fusion, particularly the genes related to the Arp2/3 complex and actin remodeling [20].

If the cells are given enough time during further differentiation to multinucleate myotubes form, one observes by fluorescence phalloidin staining that actin structures are realigned (see Figure 5.2), and the stiffness increases again. As mentioned at the beginning, cells have many possibilities to control their morphology and intracellular structures by expressing various factors [8, 9]. From this point of view, we were interested in the dynamic membrane tension generated by the cells in different stages. In the tether pulling retraction experiments, we did not observe any significant differences in 
membrane tension between the different stages. However, a general trend was observed in that the membrane tension from individual cells to matured myotubes decreased.

The transition from hemifusion-to-fusion was also suggested to be driven by fusion protein-independent forces, such as the global membrane tension. Precisely because cells go through complex changes during differentiation to control their fusion, we would like to further elucidate membrane tension during this process by examining this in the following section in a model system based on membrane-coated colloidal probes. 
A
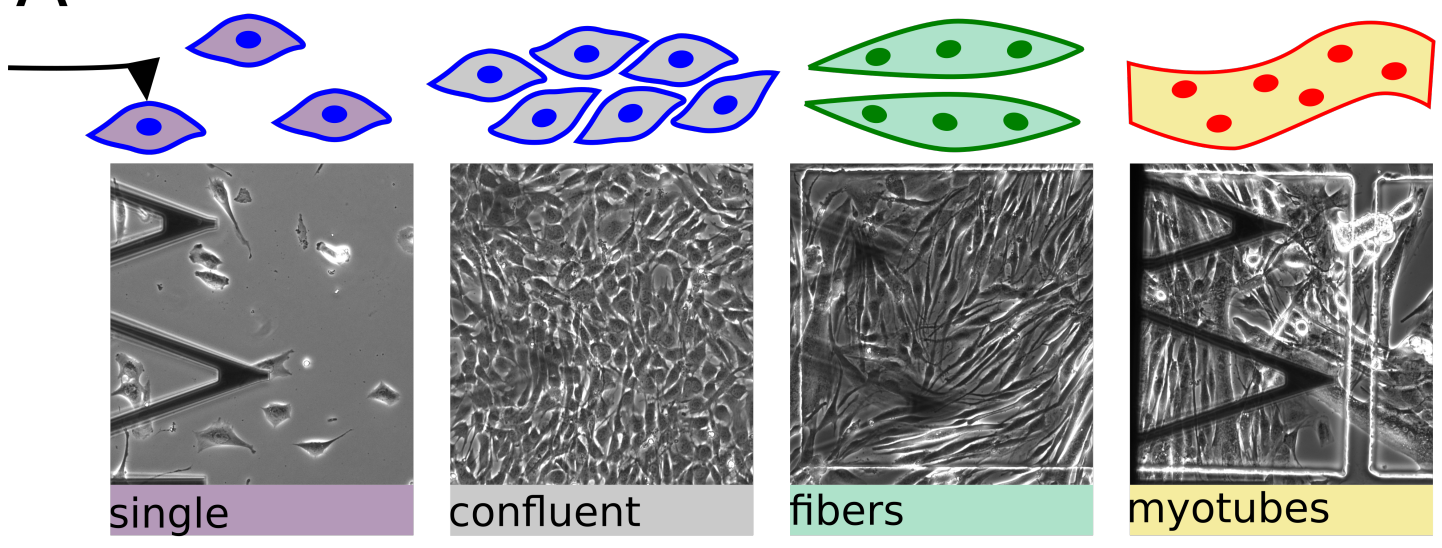

B
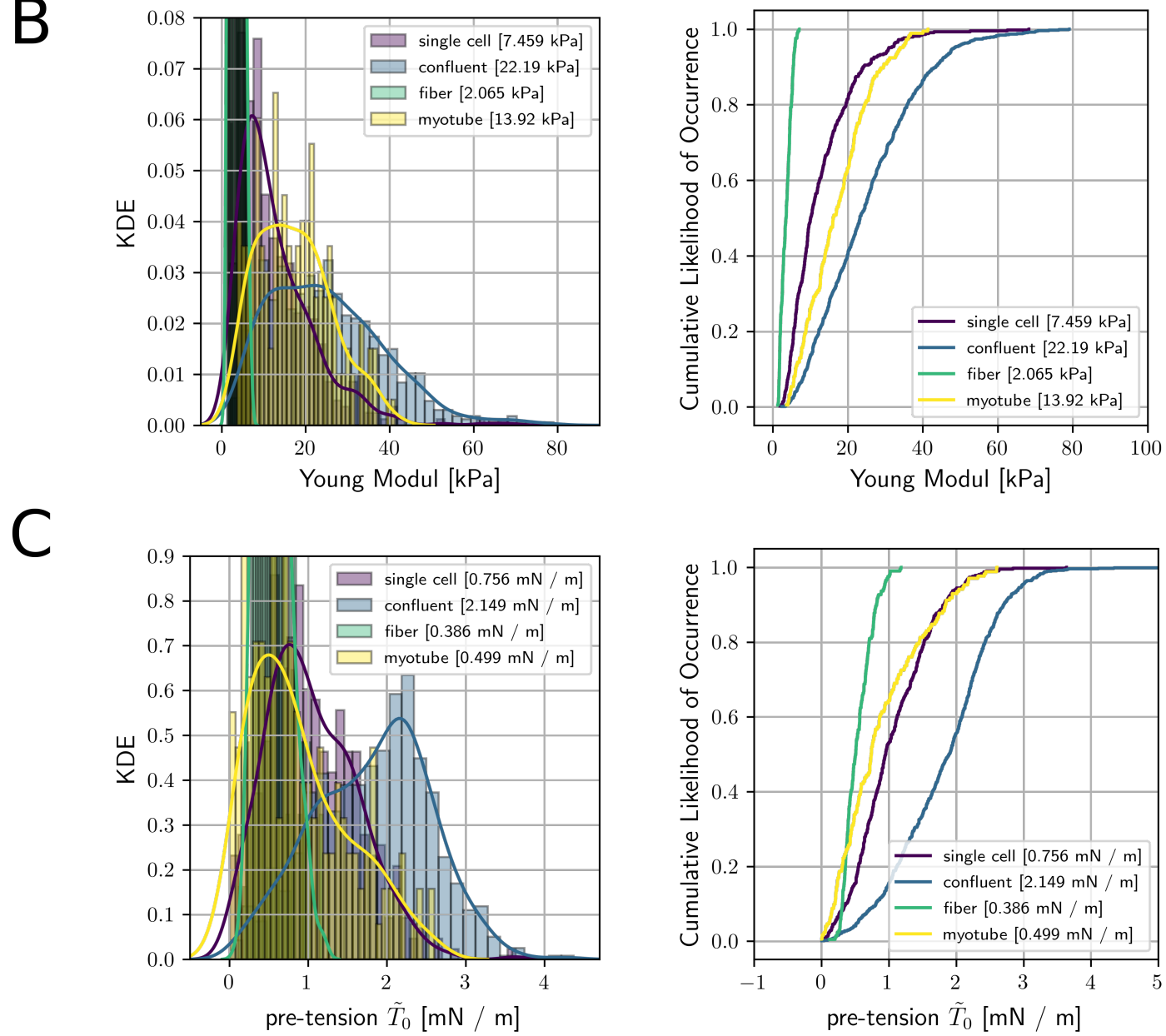

Figure 5.3: Mechanical properties of $\mathrm{C} 2 \mathrm{C} 12$ cells from indentation experiments. A: Illustrative phase-contrast images of $\mathrm{C} 2 \mathrm{C} 12$ cells in different states before and during differentiation; before indentation with a pyramidal tip on AFM-cantilever. We applied the Sneddon and tension model to the cells mechanical respose at different states: single cells before adding DM, confluent cell layer after adding DM, myoblast fibers after adding DM and matured multinucleated myotubes after adding DM. B: Histograms with KDE probability distribution (left) and cumulative distribution plots (right) of obtained Young Moduli. C: Histograms with kernel density estimated probability distribution (left) and cumulative distribution plots (right) of obtained cortical pre tension. 

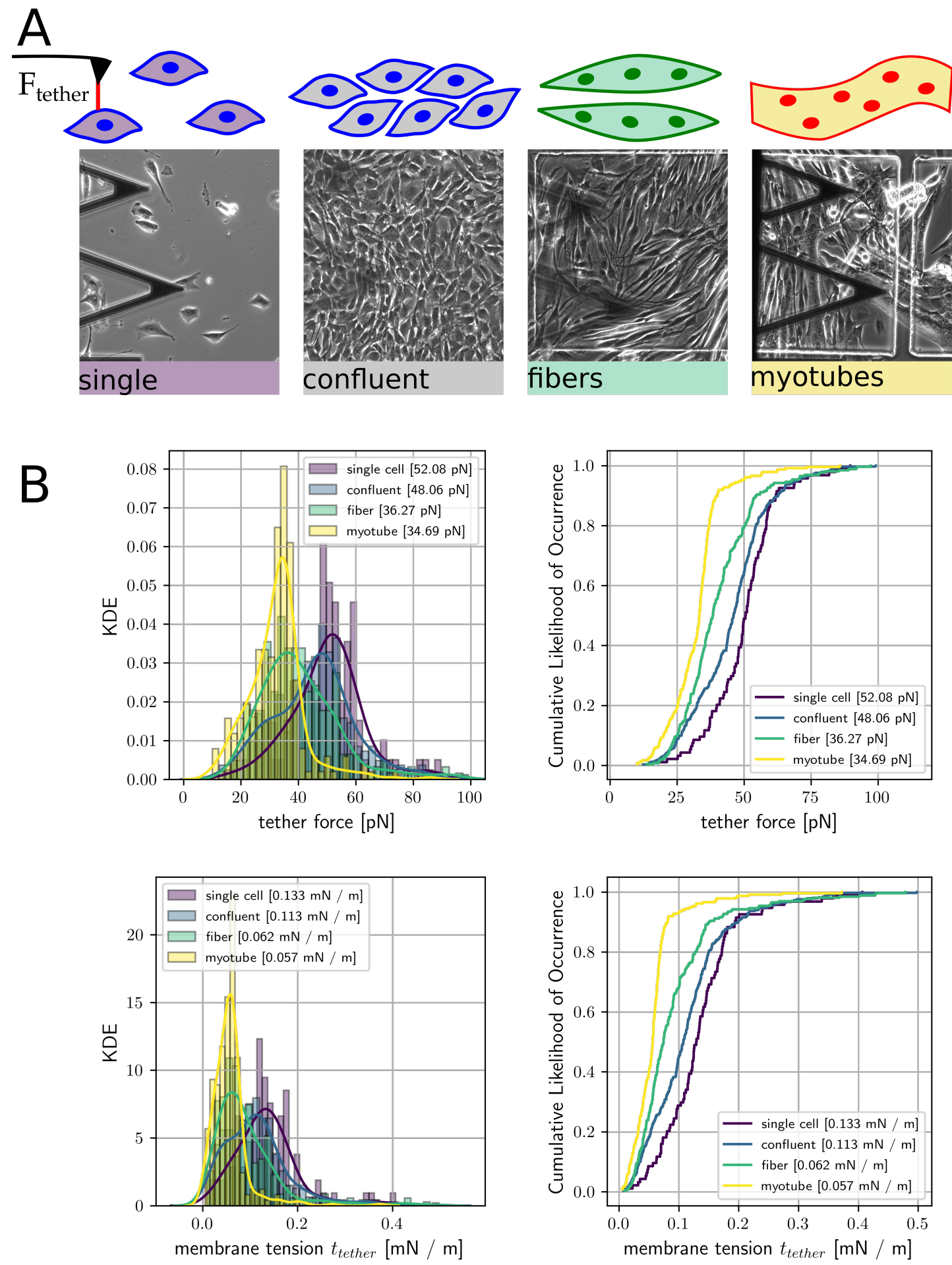

Figure 5.4: Mechanical properties of $\mathrm{C} 2 \mathrm{C} 12$ cells from retraction experiments. A: Illustrative phase-contrast images of $\mathrm{C} 2 \mathrm{C} 12$ cells in different states before and during differentiation; before retraction with a pyramidal tip on AFM-cantilever. We pull out membrane tether and monitored its rupture force at different states: single cells before adding DM, confluent cell layer after adding DM, myoblast fibers after adding DM and

B: Histograms with KDE probability distribution (left) and cumulative distribution plots (right) of obtained tether force (top) and the corresponding membrane tension (bottom). 


\subsection{Conversion into model systems based on colloidal probes}

Myoblast fusion, a critical process in the development and regeneration of the skeletal muscle, is one of the best-characterized examples of cell-cell fusion. Many proteins and lipids have been involved in myoblast fusion. Two muscle-specific proteins are essential for myoblast fusion: Myomaker and Myomerger. Myomaker is required for hemifusion, while the subsequent transition from hemifusion to complete fusion depends on Myomerger [16] . Myomerger expression in myoblasts is induced during myogenesis and is associated with the development of myoblasts' fusogenic potential. The transition from hemifusion to fusion was also suggested to be driven by proteinindependent fusion forces, such as global membrane tension [5].

In vitro fusion assays, including traditional bulk vesicle assays and single vesicle assays, reveal the molecular fusion mechanism as they allow for manipulations and observations that are not possible in vivo. A statistical investigation based on membrane-coated beads-assay in a $2 \mathrm{D}$ assembly is comparatively simple. This assay enables identifying the different phases of the fusion process which might be influenced by the presence of Myomerger.

With the bead-based assay, one can quantitatively control the composition of lipid bilayer components as we used DOPC:DOPE:DOPS:PIP2:Dye (60:18:16:5:1 mol\%, Dye: TR or Atto488) for coating bilayers on two populations of beads both incubated with different concentrations $(1,5,15,30$, and $60 \mu \mathrm{M}$ Myomerger-ectodomain in $100 \mu \mathrm{l}$ PBS buffer) of Myomerger and qualitatively characterized fusion events like docking, hemifusion, and full fusion. The populations can be distinguished by the fluorescence of an internal dye within the beads, while the fusion events can be identified by the fluorescence intensity of the bilayer at the bead's surface. Schematic illustration of scenarios after mixing membrane-coated beads labeled with Texas Red (red) and Atto488 (green) are shown in Figure 5.5A. Plain docking is followed by hemifusion and eventually by full fusion of the bilayer. The intensity analysis (Figure 5.5 B) is based on the two-channel images and is normalized to the two channels' intensity at the same pixel position [1]. The three peaks correspond to the first bead's boundary, two beads' contact zone, and the second bead's boundary. For instance, a fluorescent membrane surrounding a bead from one population (I, blue) in contact with a bead of the other population (II, red) is identified as a pure docking event. Simultaneously, a fluorescence (I) with half of the intensity found on the (II) was indicative of merging of the outer leaflet, i.e., hemifusion. Full fusion corresponds to both beads displaying equal fluorescence intensity in both channels. Efficiencies of docking, hemifusion, and complete fusion are shown in Figure 5.5D. It was found that full fusion rarely occurred. 
The advantage of this assay is that good statistics can be built up quickly and easily. Thus, in Figure 5.5D, we consider the results of the fusion bead assay of three regions of interest (ROI), 1738 beads divided into two populations of membrane coated beads in the presence of different concentrations of Myomerger per ROI. The beads were $5 \mu \mathrm{m}$ in diameter. This assay was used to answer whether to what extent the influence of Myomerger on the formation of fusion pores in cellular fusion is. We wanted to investigate whether the proportion of fully fused beads differs significantly from that of hemifusion. This is the step between pore formation and expansion or hemifusion and full fusion in cellular and artificial fusion. The experiments have shown that the addition of Myomerger to concentrations of $60 \mu \mathrm{M}$ did not increase fusogenicity. In general, the fusion events (hemifusion and full fusion) between the populations are less than $9 \%$. A slightly higher number of fusion events $6 \%$ hemifusion and $3 \%$ complete fusion) was observed for a low Myomerger concentration of $5 \mu \mathrm{M}$. This is also not surprising since Myomerger is said to influence predominantly late states of the fusion mechanism. Without hemifusion, no full fusion can be observed, which means that this proportion is deficient. Golani et al. have recently worked out how Myomerger can mainly change the membrane's intrinsic properties through a positive curvature [3] . That would have a rapid fusion rate from hemifusion to full fusion as a consequence. This is not accessible for this simple $2 \mathrm{D}$ assay. 


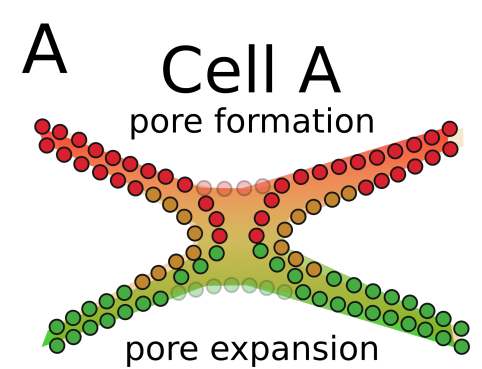

Cell B

B
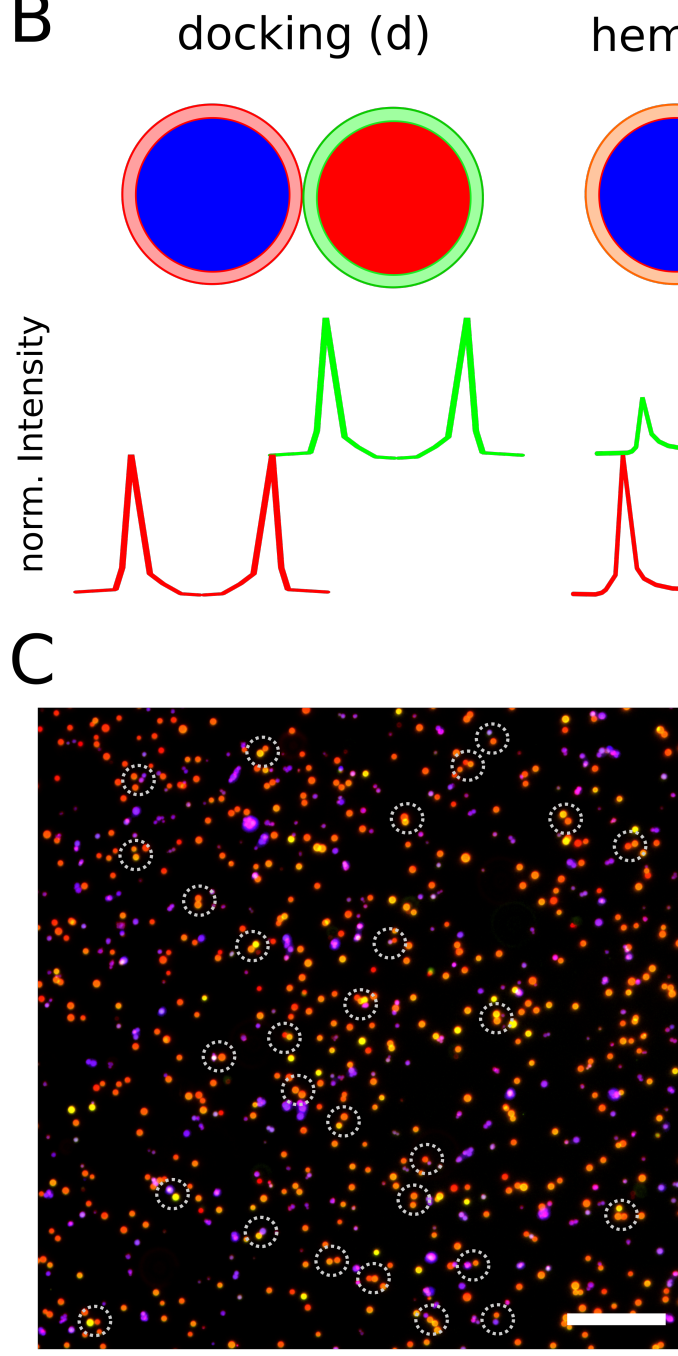

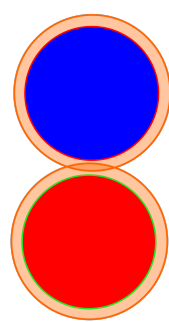

$5 \mu \mathrm{m}$
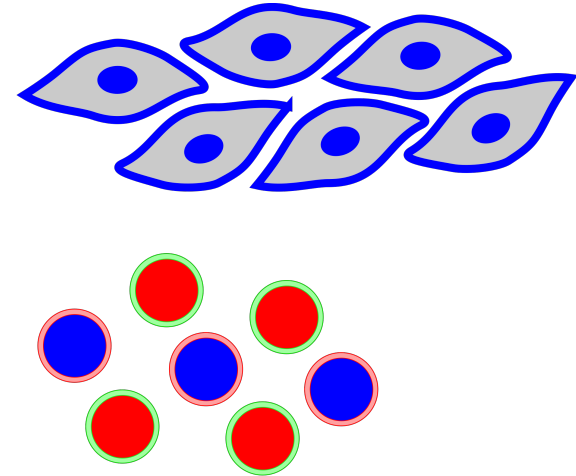

full fusion (ff)
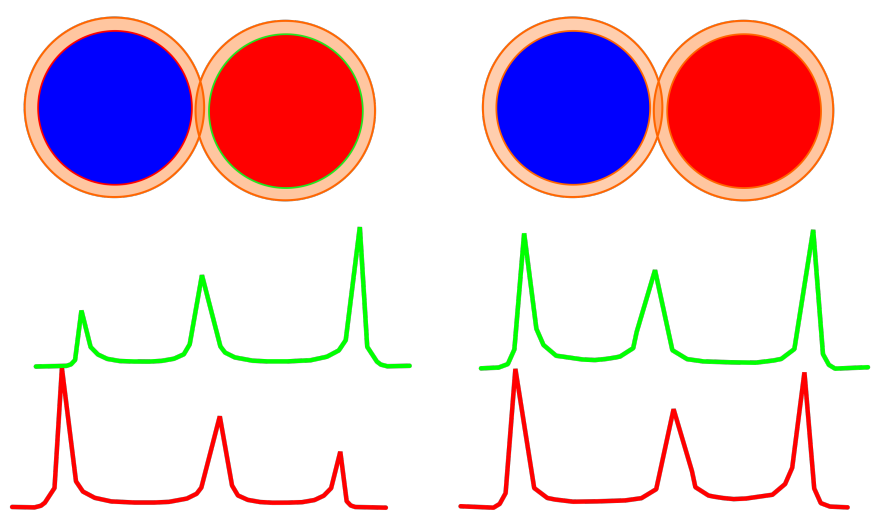

D

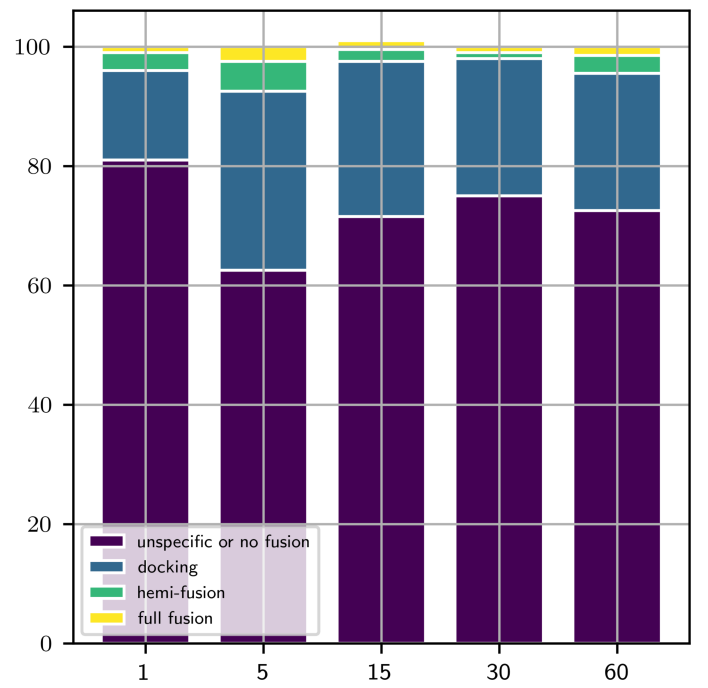

Figure 5.5: Membrane-coated beads-assay in a 2D assembly to elucidate the effect ectodomain myomerger on the mechanism of membrane fusion. A: Schematic reduction of cellular membrane fusion into model system of $2 \mathrm{D}$ assembly to study transition from pore-formation to pore-expansion. B: Schematic illustration of scenarios after mixing membrane-coated beads labeled with Texas Red (red) and Atto488 (green) together in the presence of myomerger. Distinction of fusion pairs is based on intensity analysis. C: Exemplary fluorescence image of 1738 membrane-coated beads of two population mixed together (left) and overall ratio of fusion events in dependence of myomerger concentration (right, 1-60 $\mu \mathrm{M}$ ). 


\subsection{Conclusion}

We, therefore, summarize that the mechanical properties of $\mathrm{C} 2 \mathrm{C} 12$ cells change in different stages of proliferation and the subsequent differentiation into muscle fibers through membrane fusion. Between these stages, the membrane tension is reduced, as observed through lower pull-off forces. Whether the membrane tension increases shortly before the fusion of two cells, thereby facilitating the fusion pores' expansion, is a complex process, which must be examined primarily through reduction as into an artificial model system. Here the 2D membrane-covered bead assay is only suitable to a limited extent for considering the influence of Myomerger on the fusion. However, we propose a different approach, which is presented in the next chapter in the context of SNARE-mediated membrane fusion. AFM or Optical Tweezer controlled application of membrane colloidal probes. Here, the fusion landscape's activation energy for the initial steps of the fusion could be reduced by applying a force so that the influence of Myomerger on the fusion rate from hemifusion to full fusion can then be made accessible [14, 25, 26].

\section{Reference}

[1] Chunxiao Bao, Gesa Pähler, Burkhard Geil, and Andreas Janshoff. Optical fusion assay based on membrane-coated spheres in a 2D assembly. Journal of the American Chemical Society, 2013. 135(3): 12176-12179.

[2] Alexis R. Demonbreun, Bridget H. Biersmith, and Elizabeth M. McNally. Membrane fusion in muscle development and repair. Semin Cell Dev Biol, 2015. 45: 48-56. doi:10.1016/j.semcdb.2015.10.026.

[3] Gonen Golani, Evgenia Leikina, Kamran Melikov, Jarred M. Whitlock, Dilani G. Gamage, Gracia Luoma-Overstreet, Douglas P. Millay, Michael M. Kozlov, and Leonid V. Chernomordik. Myomerger promotes fusion pore by elastic coupling between proximal membrane leaflets and hemifusion diaphragm. Nature Communications, 2021. 12(1): 1-18. doi:10.1038/s41467-020-20804-x.

[4] Jeffrey L. Hutter and John Bechhoefer. Calibration of atomic-force microscope tips. Review of Scientific Instruments, 1993. 64(7): 1868-1873. doi:10.1063/1.1143970.

[5] Ji Hoon Kim, Yixin Ren, Win Pin Ng, Shuo Li, Sungmin Son, Yee Seir Kee, Shiliang Zhang, Guofeng Zhang, Daniel A. Fletcher, Douglas N. Robinson, and Elizabeth H. 
Chen. Mechanical Tension Drives Cell Membrane Fusion. Developmental Cell, 2015. 32(5): 561-573. doi:10.1016/j.devcel.2015.01.005.

[6] Michael M Kozlov and Leonid V Chernomordik. Membrane tension and membrane fusion. Current Opinion in Structural Biology, 2015. 33: 61-67.

[7] Evgenia Leikina, Dilani G. Gamage, Vikram Prasad, Joanna Goykhberg, Michael Crowe, Jiajie Diao, Michael M. Kozlov, Leonid V. Chernomordik, and Douglas P. Millay. Myomaker and Myomerger Work Independently to Control Distinct Steps of Membrane Remodeling during Myoblast Fusion. Developmental Cell, 2018. 46(6): 767-780.e7. doi:10.1016/j.devcel.2018.08.006.

[8] Sandra B. Lemke and Frank Schnorrer. Mechanical forces during muscle development. Mechanisms of Development, 2017. 144: 92-101. doi:10.1016/j.mod.2016.11. 003.

[9] Bin Li, Michael Lin, Ying Tang, Bing Wang, and James H.C. Wang. A novel functional assessment of the differentiation of micropatterned muscle cells. Journal of Biomechanics, 2008. 41(16): 3349-3353. doi:10.1016/j.jbiomech.2008.09.025.

[10] Edward W. Li, Olivia C. McKee-Muir, and Penney M. Gilbert. Cellular Biomechanics in Skeletal Muscle Regeneration, volume 126. Elsevier Inc., 1 edition, 2018. ISBN 9780128092156. doi:10.1016/bs.ctdb.2017.08.007.

[11] Isma Mian, Willythssa Stéphie Pierre-Louis, Neha Dole, Renée M. Gilberti, Kimberly Dodge-Kafka, and Jennifer S. Tirnauer. LKB1 destabilizes microtubules in myoblasts and contributes to myoblast differentiation. PLoS One, 2012. 7(2). doi:10.1371/journal.pone.0031583.

[12] Douglas P. Millay, Dilani G. Gamage, Malgorzata E. Quinn, Yi-Li Min, Yasuyuki Mitani, Rhonda Bassel-Duby, and Eric N. Olson. Structure-function analysis of myomaker domains required for myoblast fusion. Proc Natl Acad Sci, 2016.

[13] Scott J. Nowak, Patrick C. Nahirney, Anna Katerina Hadjantonakis, and Mary K. Baylies. Nap1-mediated actin remodeling is essential for mammalian myoblast fusion. Journal of Cell Science, 2009. 122(18): 3282-3293. doi:10.1242/jcs.047597.

[14] Marieelen Oelkers, Hannes Witt, Partho Halder, Reinhard Jahn, and Andreas Janshoff. SNARE-mediated membrane fusion trajectories derived from forceclamp experiments. Proceedings of the National Academy of Sciences, 2016. 113(46): 13051-13056. doi:10.1073/pnas.1615885113. 
[15] Takahisa Okano, Satoh Shinichi, Oka Takahiro, and Matsuda Takehisa. Tissue Engineering of Skeletal Muscle.

[16] Malgorzata E. Quinn, Qingnian Goh, Mitsutoshi Kurosaka, Dilani G. Gamage, Michael J. Petrany, Vikram Prasad, and Douglas P. Millay. Myomerger induces fusion of non-fusogenic cells and is required for skeletal muscle development. Nature Communications, 2017. 8: 1-9. doi:10.1038/ncomms15665.

[17] Saravanan Rajan, Huan Chu Pham Dang, Haig Djambazian, Harry Zuzan, Yaroslav Fedyshyn, Troy Ketela, Jason Moffat, Thomas J. Hudson, and Rob Sladek. Analysis of early C2C12 myogenesis identifies stably and differentially expressed transcriptional regulators whose knock-down inhibits myoblast differentiation. Physiol Genomics, 2012. 44(2): 183-197. doi:10.1152/physiolgenomics.00093.2011.

[18] Herre Jelger Risselada, Carsten Kutzner, and Helmut Grubmüller. Caught in the Act: Visualization of SNARE-Mediated Fusion Events in Molecular Detail. ChemBioChem, 2011. 12(7): 1049-1055.

[19] Srihari C. Sampath, Srinath C. Sampath, and Douglas P. Millay. Myoblast fusion confusion: The resolution begins. Skelet Muscle, 2018. 8(1): 1-10. doi:10.1186/ s13395-017-0149-3.

[20] Gritt Schäfer, Susanne Weber, Anne Holz, Sven Bogdan, Sabine Schumacher, Arno Müller, Renate Renkawitz-Pohl, and Susanne Filiz Önel. The Wiskott-Aldrich syndrome protein (WASP) is essential for myoblast fusion in Drosophila. Developmental Biology, 2007. 304(2): 664-674. doi:10.1016/j.ydbio.2007.01.015.

[21] Ok Ho Shin. Exocytosis and synaptic vesicle function. Comprehensive Physiology, 2014.

[22] Patricia G. Spear. Virus-Induced Cell Fusion. Cell Fusion, 1987: 3-32. doi:10.1007/ 978-1-4757-9598-1_1.

[23] B. Städler, T. M. Blättler, and A. Franco-Obregón. Time-lapse imaging of in vitro myogenesis using atomic force microscopy. Journal of Microscopy, 2010. 237(1): 63-69. doi:10.1111/j.1365-2818.2009.03302.x.

[24] Zhixiong Tang, Huiling Qiu, Lan Luo, Nian Liu, Jiasheng Zhong, Kang Kang, and Deming Gou. miR-34b modulates skeletal muscle cell proliferation and differentiation. Journal of Cellular Biochemistry, 2017. 118(12): 4285-4295. doi: $10.1002 /$ jcb.26079. 
[25] Hannes Witt and Andreas Janshoff. Using Force Spectroscopy to Probe Coiled-Coil Assembly and Membrane Fusion. Springer New York, New York, NY. ISBN 978-14939-8760-3, 2018. 145-159. doi:10.1007/978-1-4939-8760-3_8.

[26] Hannes Witt, Filip Savić, Sarah Verbeek, Jörn Dietz, Gesa Tarantola, Marieelen Oelkers, Burkhard Geil, and Andreas Janshoff. Membrane fusion studied by colloidal probes. European Biophysics Journal, 2021. 50: 223 - 237.

[27] Qiao Zhang, Ajay A Vashisht, Jason O'rourke, Stéphane Y Corbel, Rita Moran, Angelica Romero, Loren Miraglia, Jia Zhang, Eric Durrant, Christian Schmedt, Srinath C Sampath, and Srihari C Sampath. ARTICLE The microprotein Minion controls cell fusion and muscle formation. Nature Communications, 2017. 8. doi: 10.1038/ncomms15664. 


\section{CHAPTER 6}

\section{SNARE MEDIATED MEMBRANE FUSION AND TRANSLATION INTO A COLLOIDAL SYSTEM}

In several fundamental biological processes, including viral infection, cell-cell fusion during fertilization, tissue development, and exo- and endocytosis, membrane fusion plays a central role [5, 20, 26, 36]. The fusion of membranes defines the process of merging two lipid bilayers in an aqueous environment. Since lipid bilayers typically do not attract each other, there is significant free energy input to overcome the fusion barriers to activation. In nature, membrane fusion is enabled by advanced proteins, through the assembling of coiled-coil structures that significantly minimize the associated energy barriers for membrane fusion [4]. These activation barriers include the removal of solvent molecules between the two opposing membranes, the splaying of lipids towards the formation of stalks, the expansion of stalks that could lead to hemifusion, and finally, the appearance of the fusion pore [29].

The main intention of this project was to obtain a detailed and quantitative image of the membrane fusion energy environment as a function of various biochemical and global physical concepts such as lipid composition, protein structure, membrane curvature, lateral strain, surface charge and presence of multivalent cations.

We accomplish this by using membrane-coated colloidal probes that combine the advantages of solid-supported membranes with complex three-dimensional geometry and ease of preparation and control. This combination makes them a robust model system that allows high-throughput and minimal reliance on fluorescent labels to visualize these complex biological processes. We demonstrate how colloidal probes can be combined with microscopy of atomic force and an optical tweezer to dissect membrane fusion topologies with great detail in spatial and temporal resolution during its pathway. Also, we demonstrate how we can measure interaction forces between 
membrane and protein as the formation of dynamic bonds of a calcium sensor, and ultimatively their effect on membrane fusion guidance or enhancement.

First we discuss different membrane topologies which can occur during the membrane fusion pathway, as shown in Figure 6.1 [5, 12]. Two separated lipid bilayers need to come into proximity during the membrane fusion process, followed by the merger of the proximal monolayers, known as hemifusion, which can then be extended and opened to establish a fusion pore. As hydration repulsion prevents membranes from coming into close contact, which is reduced by a decreased contact area, the protrusion can be described as being point-like. The hydrophilic and hydrophobic sections of the bilayer during the stalk formation form the hemifusion state. This hemifusion state is highly transient. In general, after stalk formation, two potential paths are conceivable: an immediate opening of a fusion pore or a detour involving the formation of an expanded stalk, a so-called diaphragm. This stalk hypothesis requires a free increase of energy due to stalk formation and hemifusion of $13 \mathrm{kBT}$, whereas complete fusion requires up to $46 \mathrm{kBT}$ of energy [21,22]. The core components of this fusion machinery are known to be SNAREs and can act independently of additional cytosolic accessory proteins to induce membrane fusion of vesicles.

Complete fusion events need evidence of effective lipid and content mixing. Several fusion assays using fluorescence spectroscopy were developed over time. Two separate vesicle populations are usually mixed in bulk fusion assays based on fluorescence spectroscopy, resulting in fluorescence intensity changes after effective lipid or material mixing. Membrane-dependent fluorophores or water-soluble fluorophores enclosed in the vesicle lumen are used depending on the type of fusion assay. The mixing and fusion of the vesicles' populations result in fluorophore dilution, observable by increasing fluorescence intensity.

By fluorescence microscopy of a region of interest, the fluorescence-labeled lipid bilayer covering our colloidal probe enables us to detect fusion events. This allows docking, hemifusion, and complete fusion to be quantified separately (Figure 6.2] [3]. 


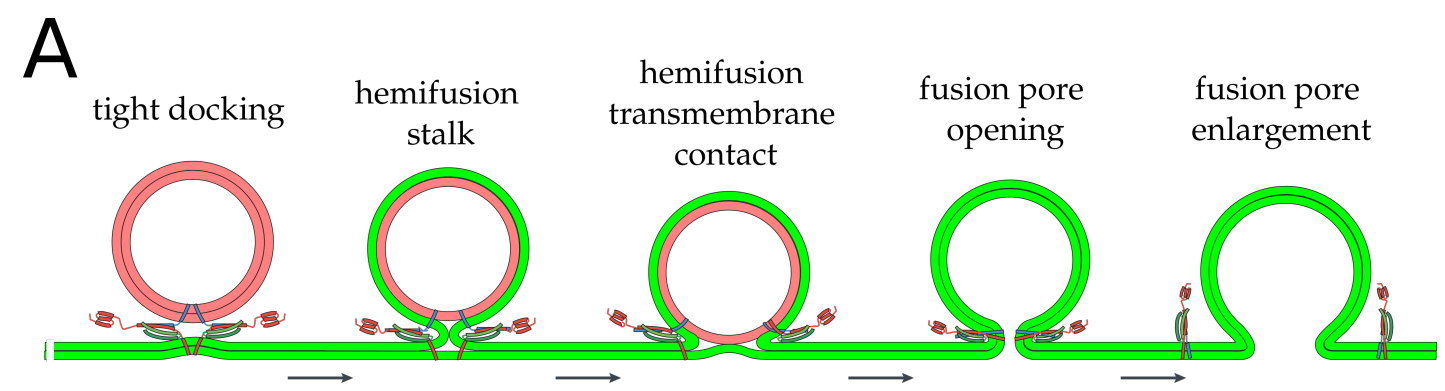

B

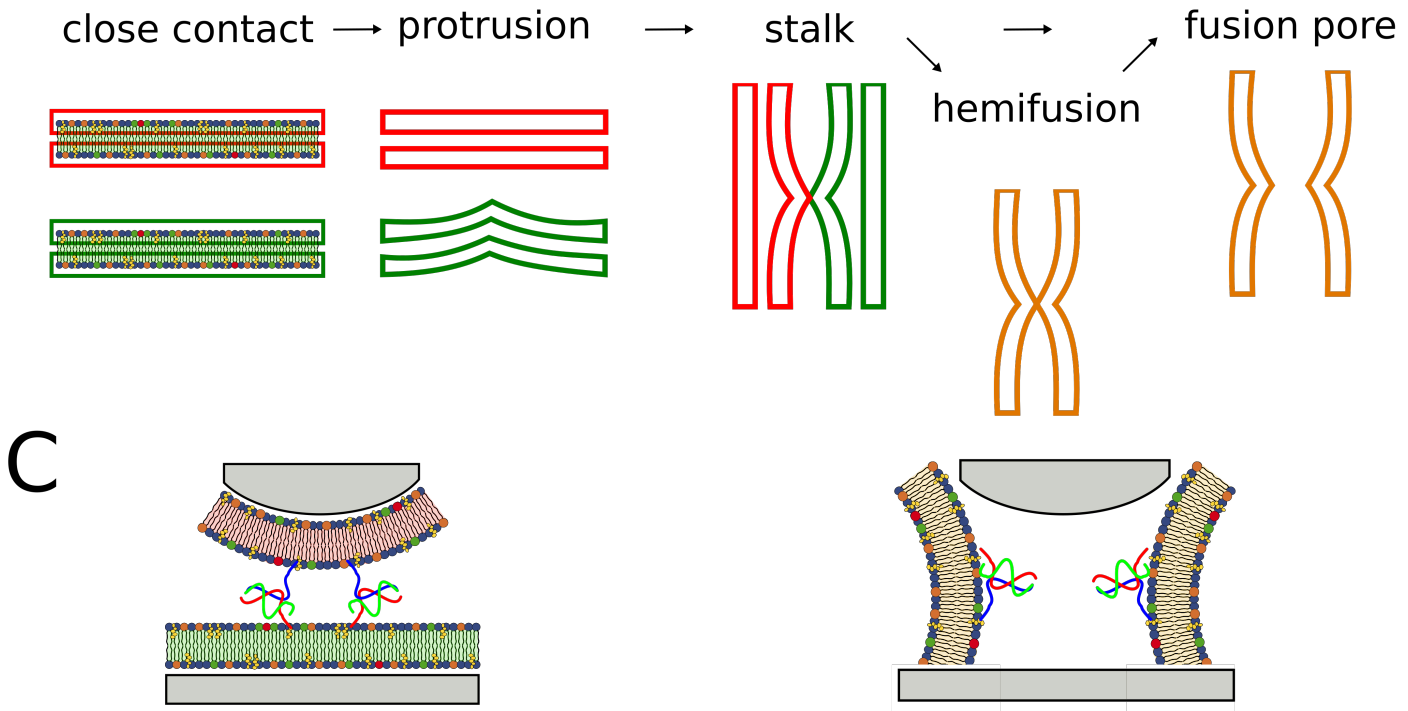

Figure 6.1: Schematic illustration of common membrane topology during membrane fusion pathway. A: Hypothetical transition states in SNARE-mediated fusion according to the stalk hypothesis on vesicular scale. B: From left to right: Membranes come into close contact until a point-like protrusion is formed. Subsequently, a hemifusion stalk is formed, which can be either expanded to a hemifusion diaphragm or open up to a full fusion pore directly. C: SNARE mediated membrane fusion. Schematic structure of the core complex consisting of syntaxin (red), synaptobrevin (blue) and SNAP-25 (green). SNARE complexes attached via transmembrane domains to bilayers on solid supports initiating membrane contact with subsequent fusion between probe and substrate. 
A

docking $(d)$

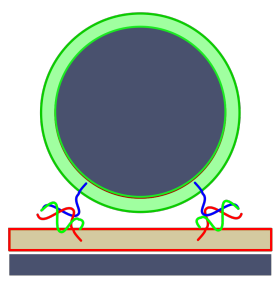

B

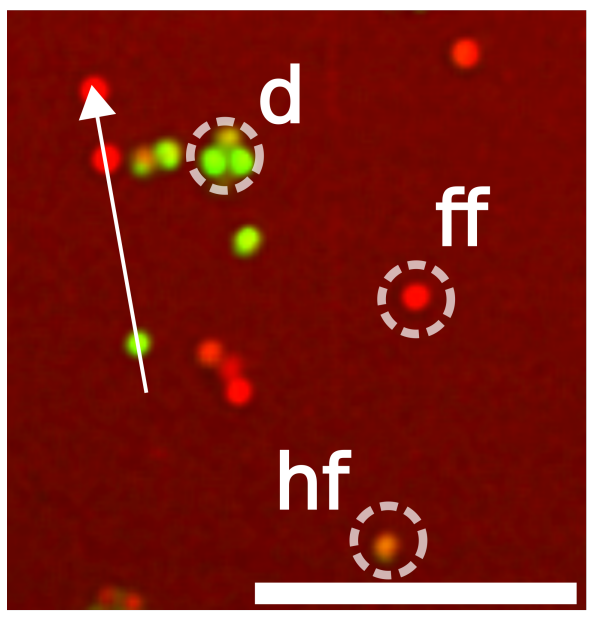

hemifusion (hf)

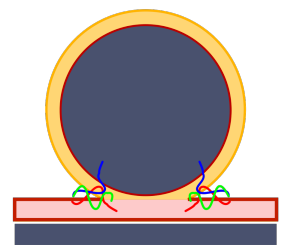

full fusion ( $f f$ )

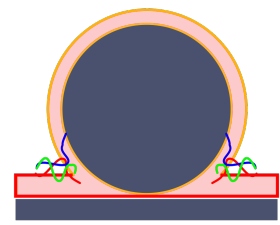

(ff)

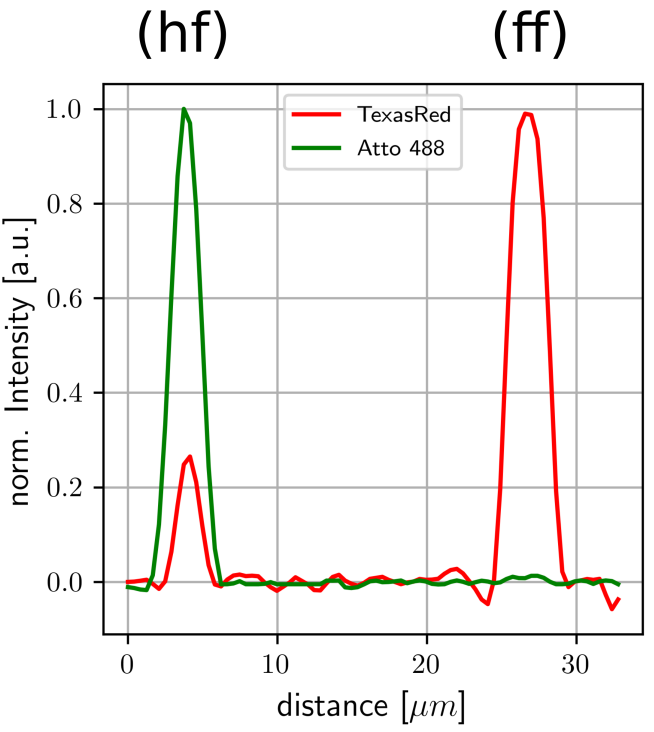

Figure 6.2: A: Schematic illustration of colloidal probes as membrane fusion assay in the presence of SNAREs. Membrane probes come into close distance before tether molecules zipper together to induce close contact between opposing membrane before resolving from hemifusion to full-fusion. B: Dual-channel fluorescence images as confirmation of lipid mixing upon membrane merging. Scale bar $10 \mu \mathrm{m}$. C: Intensity analysis using a line profile through a bead on a solid supported membrane shown in fluorescence micrograph. White arrow indicates course of analyzed line profile shown in graph. Peaks correspond to normalised fluorescence intensity. Hemifusion is shown in (hf) with a relative intensity ratio of the supported dye $\mathrm{I}_{\text {Rel }} \approx 0.5$ as expected when only the outer leaflets merge, while (ff) shows full fusion of both leaflets, $\mathrm{I}_{\text {Rel }} \approx$ is 1 . 


\subsection{Discrimination of fusion by identifying interactions and intermediates of membrane fusion mediated by SNAREs}

Colloidal probes serve as a tool to study membrane fusion in greater depth by integrating them into micromanipulation techniques such as optical tweezers or atomic force microscopy. The critical importance is that these approaches give experimental confirmation on the energy environment and the mechanical forces involved. Lipid bilayer fusion is typically stopped by significant energy barriers resulting from the hydration shell exclusion, the creation of strongly curved structures, and finally, the fusion pore expansion. Using membrane-coated silica spheres attached to an atomic force microscope's cantilevers, we measured the force-dependent lifetimes of fusion intermediates.

\subsubsection{Atomic Force Spectroscopy measurements}

This section presents membrane-coated bead colloidal probe microscopy as a label-free technique that serves to study fusion events and the lifespan of their intermediate states with subnanometer and millisecond resolution, as introduced by Oelkers and Witt et al. [29]. With a minimum of fluorescent dyes, atomic force microscopy offers a flexible way of studying membrane fusion. Additional knowledge can obtained from the deployment of external forces to discover the energy environment of membrane merging. Prominent intermediate states can therefore be allocated and quantified statistically.

The experiment was performed, as prepared in chapter 3.6 and results shown in Figure 6.3. Until a given force is achieved, two bilayers are brought into contact. The atomicforce microscope force feedback is turned off, and the cantilever deflection is tracked as a function of time after the yield force was achieved. In the presence of SNAREs, one observes that several nanometers of cantilever deflection leap stochastically within milliseconds. These steps can be assigned as fusion intermediates. Analysis of this data reveals step height distributions containing prominent recurrent fusion intermediates. The fusion of only two leaflets, such as resolves hemifusion in 2.1-4.2 $\mathrm{nm}$ and merger of one-step full fusion of outer and inner leaflets in 4.2-8.4 nm, distinguished by step heights corresponding to the removal of a single bilayer or two bilayers, respectively, are the most specific motions [29].

This approach shows that stalk formation, hemi-fusion, and complete fusion can be differentiated from the formation of the SNARE complex, and the fusion trajectories as the underlying energy landscape can be precisely reconstructed by calculating 
the lifetime distributions of intermediate states $\tau_{\text {life }}$ between fusion times $\tau_{\mathrm{d} 2 \mathrm{f}}$ in the time range of lower than a few hundreds of ms and the corresponding step height distribution $\delta_{\text {step }}$.

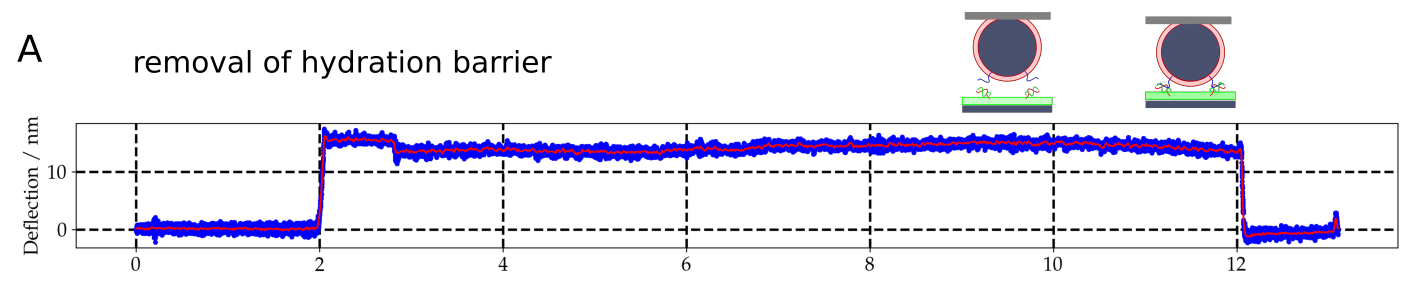

formation of hemifusion stalk
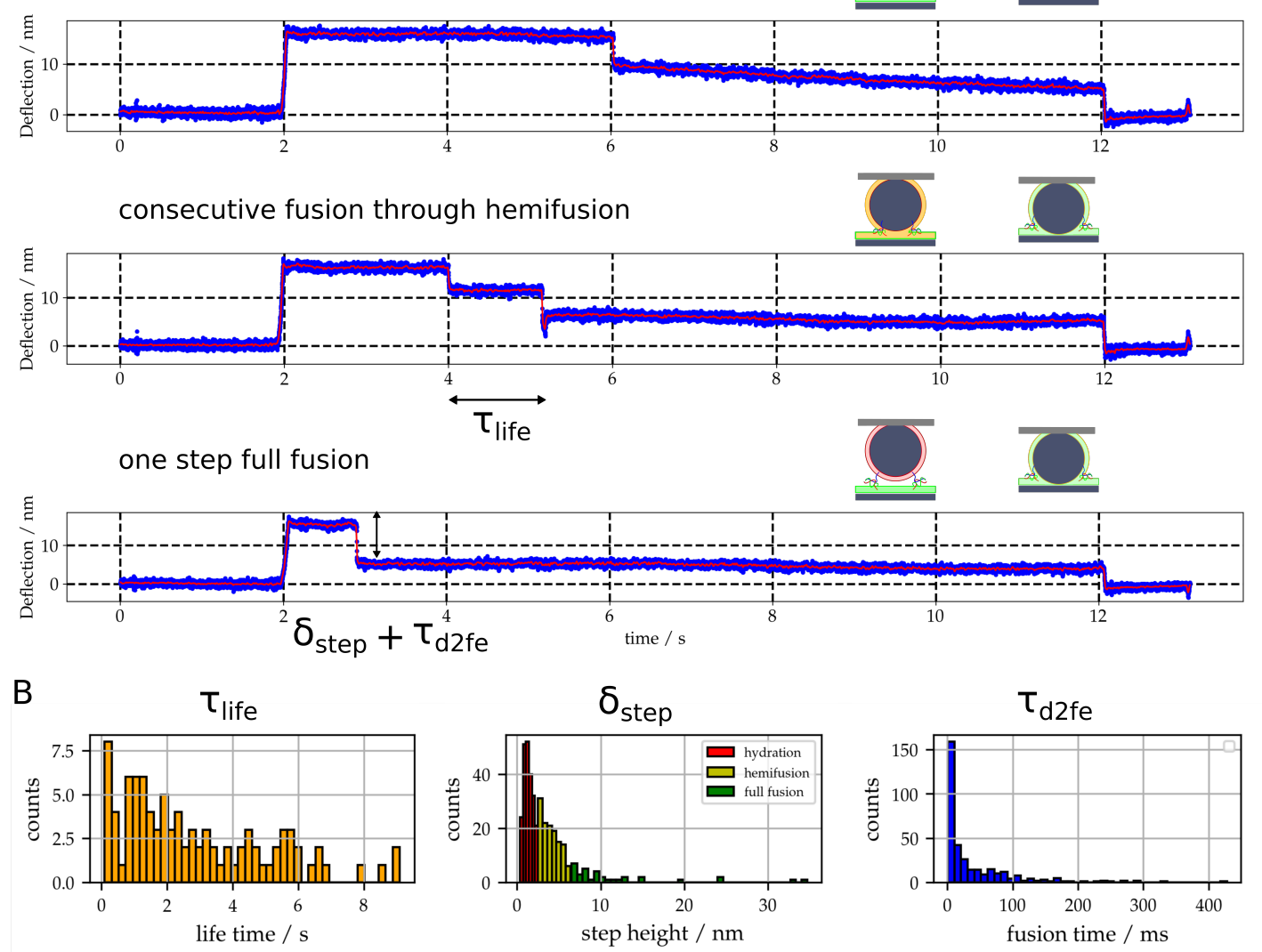

Figure 6.3: A: Illustration of the AFM setup equipped with the colloidal probes. The colloidal probe is covered with a lipid bilayer and brought into contact with the bilayer on the opposing planar surface, and both membranes reconstituted with native fusion proteins as indicated. Typical time traces are shown in the fusion experiment. Relevant transitions states to fusion intermediates can be identified by different step heights. SNARE complex formation, docking or removal of hydration barrier: 1-2 nm, hemifusion $4 \mathrm{~nm}$, and complete fusion 8-10 $\mathrm{nm}$ are observable fusion events. B: Fusion Landscape Histograms derived from AFM-CP time traces. Parameters obtained are lifetime $\tau_{\text {life }}$ between two individual steps $\approx 0.1-8 \mathrm{~s}$, step height distribution allocated to intermediates (hydration barrier $\approx 2 \mathrm{~nm}$, hemifusion $\approx 4 \mathrm{~nm}$, and full-fusion $\approx 8 \mathrm{~nm}$ ) and time past for individual steps from docking to full-fusion $t a u_{\mathrm{d} 2 \mathrm{f}} \approx 0.2-200 \mathrm{~ms}$.

As a function of external force, Witt et al. could demonstrate that force clamp tests help 
detect fusion events and calculate intermediate states, rates and its lifespan. Such states, such as the initial barrier crossing, but not all, are accelerated by external elastic energy, e.g., the phase from hemifusion to complete fusion [29, 44]. We discuss the distances involved in membrane fusion aided by SNARE proteins within these circumstances.

\subsubsection{Optical Tweezer measurements}

To understand the biological role of the involved proteins, a comparative evaluation of the free energy landscape of membrane fusion in the absence and existence of parts of the fusion machinery is of vital importance. Although theoretical studies are gradually converging towards a similar qualitative and quantitative image, experimental evidence is scarce mainly due to technical difficulties and the lack of adequate model systems. In this example, we are studying the influences of membrane fusion aided by SNARE proteins or external energy input. In this situation, optical tweezers have the required force resolution to catch the tiniest events associated with dezippering and crossing of the hydration barrier of coiled-coil complexes. During the past years multiple advantages in this field have been made.

Oddershed et al. conducted vesicle membrane modulation by optical tweezers [1]. Using a directed laser beam, thin unilamellar lipid vesicles with diameters down to $50 \mathrm{~nm}$ enclosing high refractive index sucrose cores may be optically trapped in three dimensions individually. Simultaneous quantitative measurements of the forces exerted on individual vesicles and their scale and structure are made possible by combined optical trapping and confocal microscopy. With nanometer spatial and $10 \mu$ s temporal resolution, individual vesicles' location in three dimensions is measured. In optical tweezers, a small unilaminar vesicle with an aqueous center is deformed at high laser power. As a result of the laser-induced surface stress, the vesicle could be found to undergo a budding transformation.

Yuval Elani et al. have developed controlled vesicle fusion experiments and sculpting biomimetic vesicle networks using optical tweezers [1]. At least two different liposomes are brought into contact by optical tweezers. A pulsed UV laser initiates the membrane's disruption in the contact interface, and all liposomes resolve into a single vesicle.

A flexible microfluidics framework for nanomanipulation and mechanical characterization of artificial cell membranes interfaced with optical tweezers was developed and implemented by Aubin-Tam et al.: A free-standing membrane composed of a mixture of dioleoylphosphatidylcholine (DOPC) and dipalmitoylphosphatidylcholine (DPPC) between two microchannels of a flow cell is formed there [6]. They combine optical 
tweezers with a flow cell that provides autonomous access to both membrane leaflets. The system offers independent and complex control over either side of the membrane's physiological conditions. The flow cell facilitates electrophysiology measurements, which can be used for tracking the membrane capacitance in real-time. The combination of free-standing membranes with optical tweezers causes the lipid bilayers to form nanotubes through nanomanipulation.

Zhang et al. and Keidel et al. have done the most similar studies to our approach [14, 45]. The first introduces an experimental system that uses dual-trap high-resolution optical tweezers and microfluidics to investigate controlled SNARE folding and assembly. A single SNARE complex is attached with a $2.260 \mathrm{bp}$ DNA handle to two polystyrene beads and pulled in the absence or presence of $\alpha$-SNAP in the solution. Two kinds of signals are obtained: curves of force-extension and trajectories of time-dependent extension at constant separations of traps or mean forces. Information on SNARE folding and unfolding transitions and $\alpha$-SNAP binding and unbinding have been extracted from these measurements. Besides, for synaptotagmin-1 (soluble Syt-1) and extended synaptotagmin-2, they performed this approach as a single-molecule force spectroscopy technique to measure membrane binding of $\mathrm{C} 2$ domains. $\mathrm{C} 2$ domains resisted unbinding forces of 2-7 $\mathrm{pN}$ from either protein and had binding energies of 4$14 \mathrm{kBT}$ per domain $\mathrm{C} 2$. The established properties of both proteins are recapitulated by control of bilayer composition or $\mathrm{Ca}^{2+}$. For analyzing protein-membrane interactions, the technique can be generally used. It is often difficult to research these interactions, mainly when they include multiple intermediates, multiple ligands, mechanical force, significant changes in energy, or protein aggregation.

Keidel, Bartsch and Florin introduce an assay on silica beads and coverslips for membrane fusion of solid assisted membranes [14]. Lipid bilayer fusion is provoked by getting an optically trapped bead into contact with a bilayer on a coverslip surface while observing the bead's thermal motion using the three-dimensional location detector, microsecond temporal, and nanometer spatial resolution. They demonstrate that shifts in the bead's three-dimensional position histograms and its diffusion rate will follow fusion progression. Any fusion intermediates, including transient fusion, stalk development, hemifusion, and a fusion pore completion, were observed. Connected to our AFM experiments, fusion intermediates are distinguished by axial and not lateral confinement of the bead's motion and independently by the shift in its diffusion rate due to the additional drag between the two membranes from the stalk-like connection. 


\section{Dissecting landscape of membrane fusion by optical tweezer measurements}

Comparing different strategies to realize Colloidal Probe or Force Spectroscopy experiments, in general, one notices one same principle. A probe is attached to a spring with a known spring constant $k_{c}$ allowing us to calculate the force $F$ by measuring the displacement $\Delta z$ of a spring using Hooke's law $F=k_{c} \Delta z$. The contrast between different force spectroscopic methods is how it realizes the spring and how $\Delta z$ is determined [9, 11, 13, 27, 40]. For typical cantilever one obtains spring constants in the range of 10-15 pN/nm, while optical trap stiffness is much lower. As shown in Figure 6.4 one obtains spring constants in the order of $0.35 \mathrm{pN} / \mathrm{nm}$ at maximum use of LASER power. In the low stiffness regime, one measures mostly confined motion with changes in the position distribution. In contrast, in high stiffness regime, one measures step-wise displacements down to a few nanometers (Figure 6.5), likewise obtained in our AFM approach.
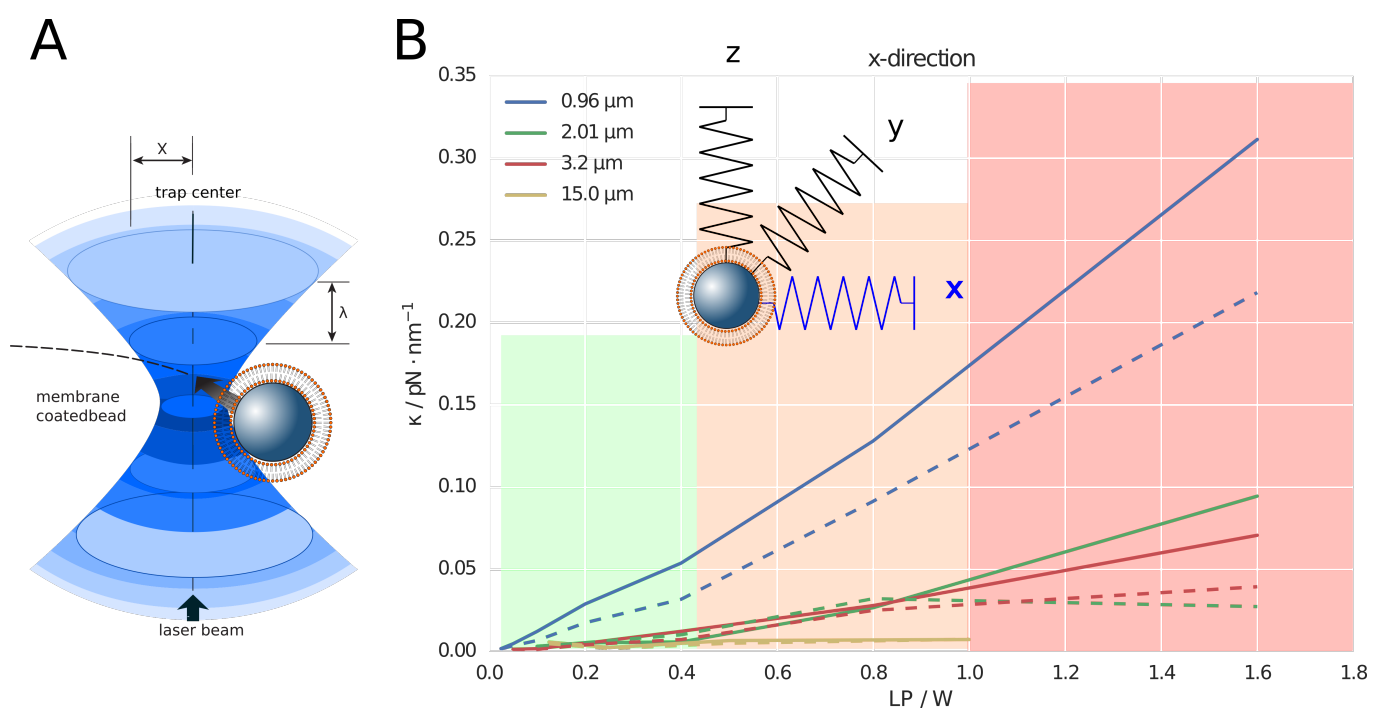

Figure 6.4: Overview of Membrane Coated Colloidal Probes Force Spectroscopy by Optical Tweezer. A: Schematic representation of the setup. The NanoTracker 2 optical tweezer (JPK Instruments, Berlin, Germany) build on an inverted Zeiss microscope equipped with a $5 \mathrm{~W} \lambda=1064 \mathrm{~nm}$ LASER and a water immersion objective (63x, CApochromat, NA = 1.2, Carl Zeiss, Jena, Germany). B: Spring constants of membrane coated silica beads as a function of applied LASER power for 4 different bead diameters.

We performed two approaches to identify, characterize, and distinguish fusion intermediates from protein-membrane interactions and adhesion of small membrane coated beads (1-5 $\mu \mathrm{m}$ diameter) with high and low spring constants. Figure 6.5 demonstrates a setup where a $1 \mu \mathrm{m}$ membrane-covered bead is brought into contact with a $15 \mu \mathrm{m}$ membrane-coated bead by the piezo controlled moving of the stage and the underlying substrate, which is a $15 \mu \mathrm{m}$ membrane-coated bead covalently bond to a PDMS substrate. Once contact is established, we observe a 2-stepwise shift in the mean displacement, 
which we correlate to previously assigned fusion intermediates. After these events have occurred, we were able to pull out a single membrane tether, which has been formed and elongated after fusion. Furthermore, one has to consider that at high laser powers and high trap stiffness it is most likely that fusion is accelerated by the LASER beam's external energy input. To obtain a comparable result, we furthermore performed additional experiments in the low stiffness regime, as shown in Figure 6.6. Here fusion is observed by reduced motion and lateral confinement, as previously observed by Keidel, Bartsch, and Florin.
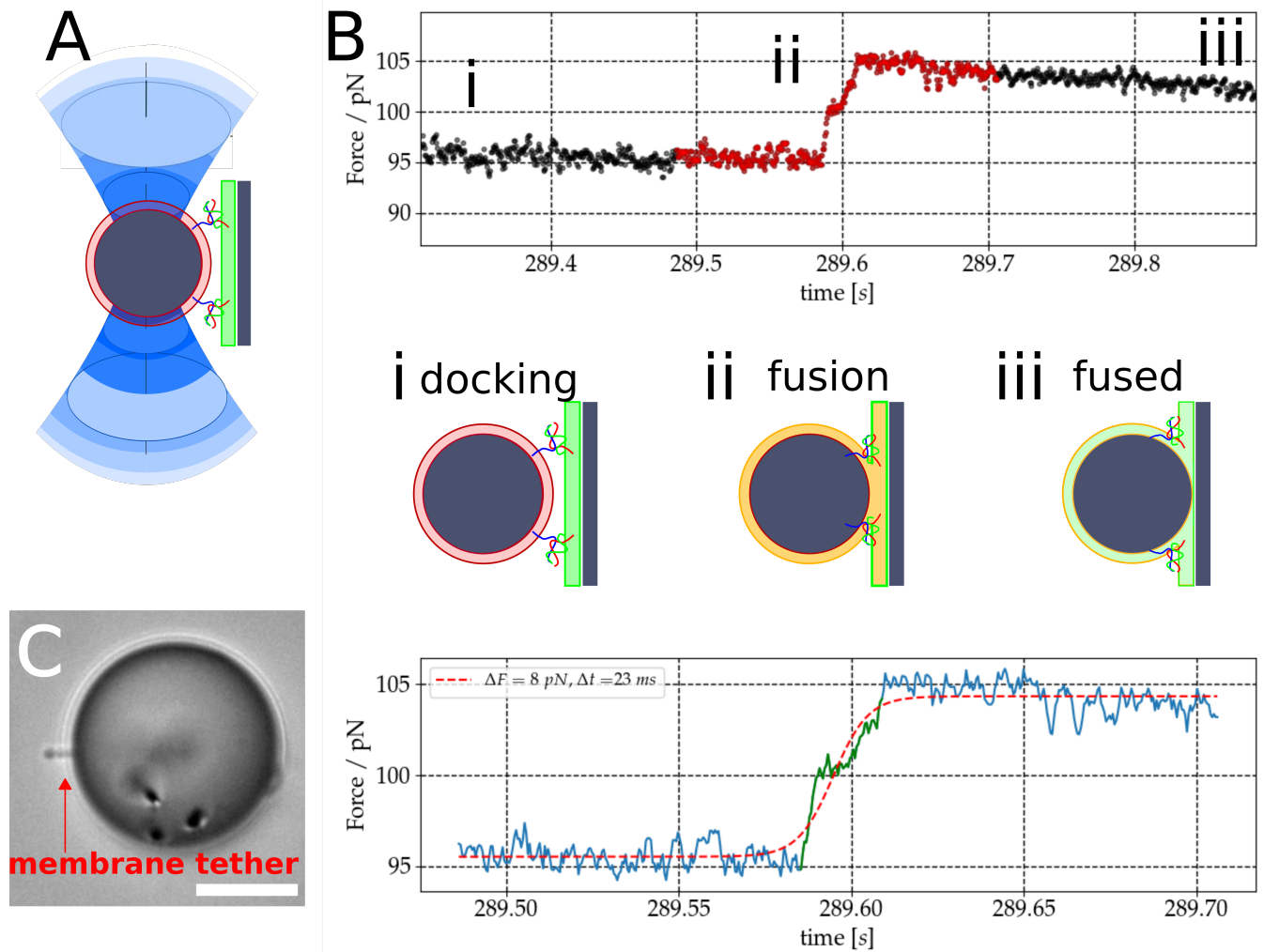

Figure 6.5: Overview of membrane coated colloidal probes force spectroscopy by optical tweezer. $1 \mu \mathrm{m}$ membrane-covered bead comes into contact with a $15 \mu \mathrm{m}$ membranecoated bead by OT. Membrane consists of DOPC:DOPE:DOPS:Chol:Dye-(Atto488 or TR)-DHPE (mol\%, ratio 55:22:11:11:1). A: Schematic representation of the setup. B: Representative time trace obtained from the fusion experiment. Range in which fusion step occurs was fitted with a generalized sigmoidal function to obtain step height and fusion time, as shown in the bottom plot. C: Bright field image of the experiment after retraction. Image shows lipid nanotube between colloidal probe in the optical trap and the supported bilayer. Scale Bar $7.6 \mu \mathrm{m}$

Figure 6.6 displays a second approach. Where a planar substrate with patterned membrane patches was used. The actual fusion events are detected and analyzed by measuring membrane-coated bead motion when brought in near contact with the patterned bilayers. Supported is a membrane patch containing the complement SNARE protein 
triggering membrane fusion. The bead is held above a membrane patch at a laser beam's fixed position, while the substrate is moved upwards via a piezo-stage, establishing contact between both membranes. Membrane fusion is then captured with microsecond resolution by monitoring the bead's displacement from its trap-center in $\mathrm{x}-, \mathrm{y}-$ and $\mathrm{z}$-direction. After a successful fusion, displacement of the bead in the lateral confinement is significantly decreased. Time-traces detect and reject multiple fusion events from hemifusion, docking to absolute fusion. As pictured above, we confirm that force-clamp experiments are effective to identify fusion events.

Furthermore, we analyzed time traces obtained from these experiments by PCA and PELT changepoint detection to automatically obtain location when the particle movements' motion in the optical trap changes. With that, we assign autonomously confined motion radii of the trapped colloidal probe to specific fusion events like docking, hemifusion, and full fusion by evaluating the 2-dimensional MSDs as shown in Figure 6.7 C (i) $24-30 \mathrm{~nm}$ (ii) $11-39 \mathrm{~nm}$ (iii) 4.5-5.4 nm (Supporting Figure 50 6.12). As indicated before, external elastic energy promotes some steps, such as the first boundary crossing (hydration barrier), but not the phase from hemifusion to total fusion. It is most likely that other membrane protein interactions are needed to accelerate later stages of fusion as hypothesized for the ectodomain of Myomerger in cellular membrane fusion. Nevertheless, to summarize, we note that the fusion of membrane coated colloidal probes is accompanied by a significant reduction in particle motion or confinement, which OT or HVPT can detect. Therefore we will use motion radii to discriminate protein-membrane and membrane-membrane interaction from fusion events in an almost force-free environment. We are deciphering synaptotagmin's role, and $\mathrm{PI}(4,5) \mathrm{P}_{2}$ 's influence on the mechanism of SNARE mediate membrane fusion in the following section. 

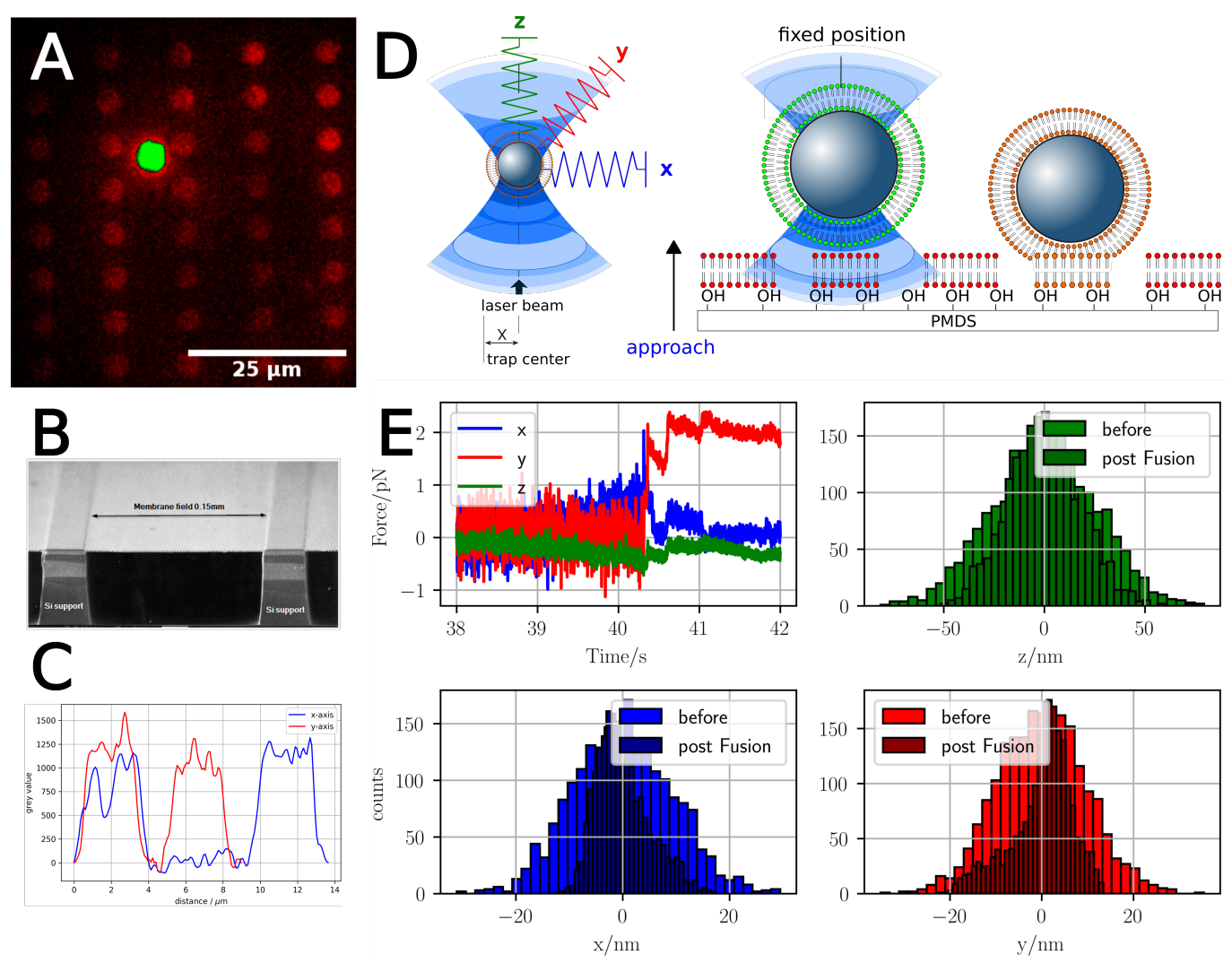

Figure 6.6: Assay to quantify SNARE-mediated membrane fusion using patterned membrane patches on oxidized PDMS supports. A: Membrane patches (red) were formed by spreading small unilamellar vesicles (SUVs) on hydrophobic patterns of PDMS bonded to glass, while a single membrane coated bead (green) was caught in an optical trap. B: Silica micro sieve chips with pore sizes of $5 \mu \mathrm{m}$ processed by Aquamarijn used to create hydrophobic PDMS patterns by oxygen-plasma activation of cured PDMS. C: Line plot of a representative fluorescence image of membrane patches. Shown are vertical (y-axis), and horizontal (x-axis) distances between membrane patches of 5 $\mu \mathrm{m}$ in size. A repetitive pattern of patches containing reconstituted SNARE proteins in a confined region is formed. D: Schematic illustration of the experimental approach. A silica microbead coated with a fluorescent-labeled membrane (green) containing reconstituted SNARE complexes is caught in an optical trap through a transparent PDMS layer of $100 \mu \mathrm{m}$ thickness bonded to a glass surface. On top is a membrane patch (red) containing the complement SNARE protein triggering membrane fusion. The bead is held above a membrane patch at a fixed position of the laser beam, while the substrate is moved upwards via a piezo-stage, establishing contact between both membranes. Membrane fusion then is captured with microsecond resolution through monitoring the displacement of the bead from its trap-center in $\mathrm{x}-, \mathrm{y}$ - and $\mathrm{z}$-direction. E: Timetraces of a representative fusion process and bead-position histograms before and after membrane fusion. The time-trace shows consecutive steps after contact representing docking and subsequent fusion of the underlying bilayer. Once fused the bead moves in a confined environment reducing its movement within the laser beam. 

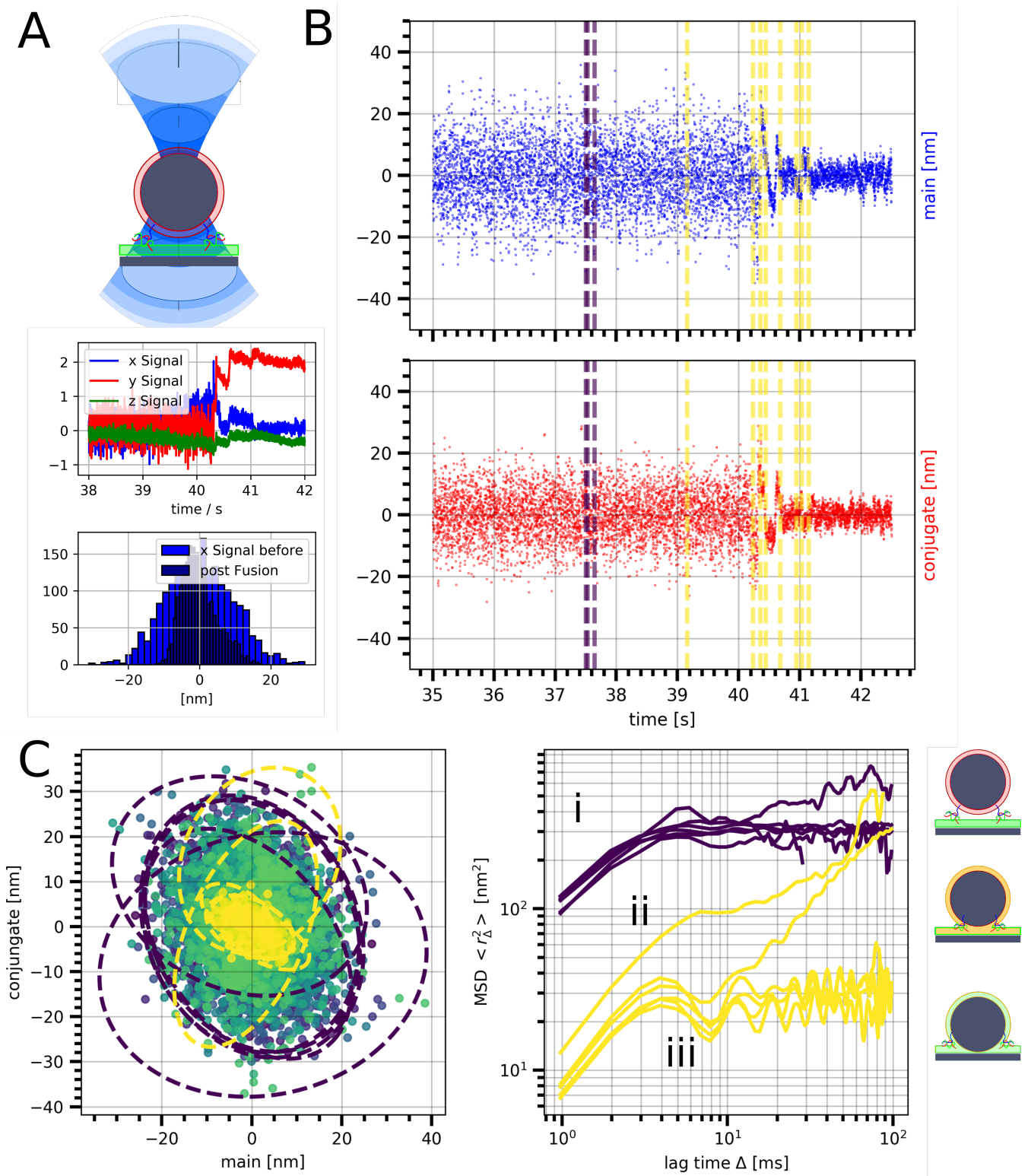

Figure 6.7: Illustrative result of membrane coated colloidal probes fusion assay by optical tweezer. A: Schematic representation of the setup. Time-traces of a representative fusion process and bead-position histograms before and after membrane fusion. The time-trace shows consecutive steps after contact representing docking and subsequent fusion of the underlying bilayer. Once fused the bead moves in a confined environment reducing its movement within the laser beam. B: Segmented particle motion of trapped probe. A PELT detection algorithm was applied on experimental data after PCA. C: States forwarded from (B) are analysed with MSD as function of time. 3 different confinement radii are observed: (i) $24-30 \mathrm{~nm}$ (ii) $11-39 \mathrm{~nm}$ (iii) $4.5-5.4 \mathrm{~nm}$. 


\section{Probing protein-membrane interactions for membrane fusion by optical tweezer measurements}

For investigating protein-membrane interactions, Zhang et al. showed that one could generally use optical tweezer techniques [45]. It is often difficult to research these interactions, mainly when they include multiple intermediates, multiple ligands, mechanical force, significant energy changes, or protein aggregation. Investigating properties associated with membrane-membrane and membrane-protein interactions caused by Syt-1 or Doc2B, Sorkin et al. used a dual trap approach [35]. They could inquire about membrane-membrane interaction and strength in these proteins' presence by taking phospholipid bilayer-coated beads into contact and subsequently pulling them apart. In this approach, this behavior is compared with that of Doc $2 b$, which includes a conserved $\mathrm{C} 2 \mathrm{AB}$ domain and causes membrane tethering and hemifusion. In a purely $\mathrm{Ca}^{2+}$ and protein-dependent way, they find that the soluble C2AB domain of Syt-1 significantly affects the frequency and strength of membrane-membrane interactions. These findings suggest that in the presence of $\mathrm{Ca}^{2+}$ sensors, OT-technique serves to monitor membrane-membrane interactions. Both fragments of $\mathrm{C} 2 \mathrm{AB}$ reliably induce a decrease in the membrane-bending modulus. This reduction of the energy needed for membrane deformation can lead to the fusion induced by $\mathrm{Ca}^{2+}$.

It is well-established that exocytosis of synaptic vesicles is triggered by $\mathrm{Ca}^{2+}$. Identification of the $\mathrm{Ca}^{2+}$ sensor that regulates the fusion reaction opened the door to tracing this transduction pathway, thus providing a means of elucidating the mechanism of regulated exocytosis. A 65-kDa protein, Syt, localized to synaptic vesicles was found in neurons and neuroendocrine cells. The primary sequence of Syt- 1 revealed a single membrane-spanning domain, a short intraluminal domain, and a large cytoplasmic domain consisting of tandem $\mathrm{C} 2$ domains, $\mathrm{C} 2 \mathrm{~A}$ and $\mathrm{C} 2 \mathrm{~B}$, connected by a linker. This protein is believed to bind specifically to phosphatidylinositol-4,5-bisphosphate $\left(\mathrm{PI}(4,5) \mathrm{P}_{2}\right)$ making up more than $80 \%$ of the total surface area in syx-1A microdomains [42]. It was found that $\mathrm{PI}(4,5) \mathrm{P}_{2}$ specifically connects the C2B domain of Syt-1 and syx-1A in a $\mathrm{Ca}^{2+}$-independent manner based on the polyanionic headgroup of $\mathrm{PI}(4,5) \mathrm{P}_{2}$ and the polybasic sequences in syx-1A (260-KARRKK-265) and C2B (324-KKKK-327) [25]. We now try to quantify the impact of $\mathrm{PI}(4,5) \mathrm{P}_{2}$ on fusion efficiency and lifetime of the various intermediates. For this purpose, we use a reconstituted system consisting of SNARE proteins and Syt-1 to imitate the situation of the synaptic vesicle and supported bilayers equipped with $\mathrm{PI}(4,5) \mathrm{P}_{2}$ and PS to enable the fusion triggered by $\mathrm{Ca}^{2+}(100 \mu \mathrm{M})$. The primary Syt-1 binding results is seen in Figure 6.8 .

Clearly, in the absence of protein, Syt-1 raises adhesive forces relative to the membranemembrane interaction. We are now investigating the effect of Syt-1 recognition by 
$\mathrm{PI}(4,5) \mathrm{P}_{2}$ on membrane fusion. Time traces of the bead-distance would allow us to recognize the fusion event by an immediate decrease in the motion when the membrane merges.
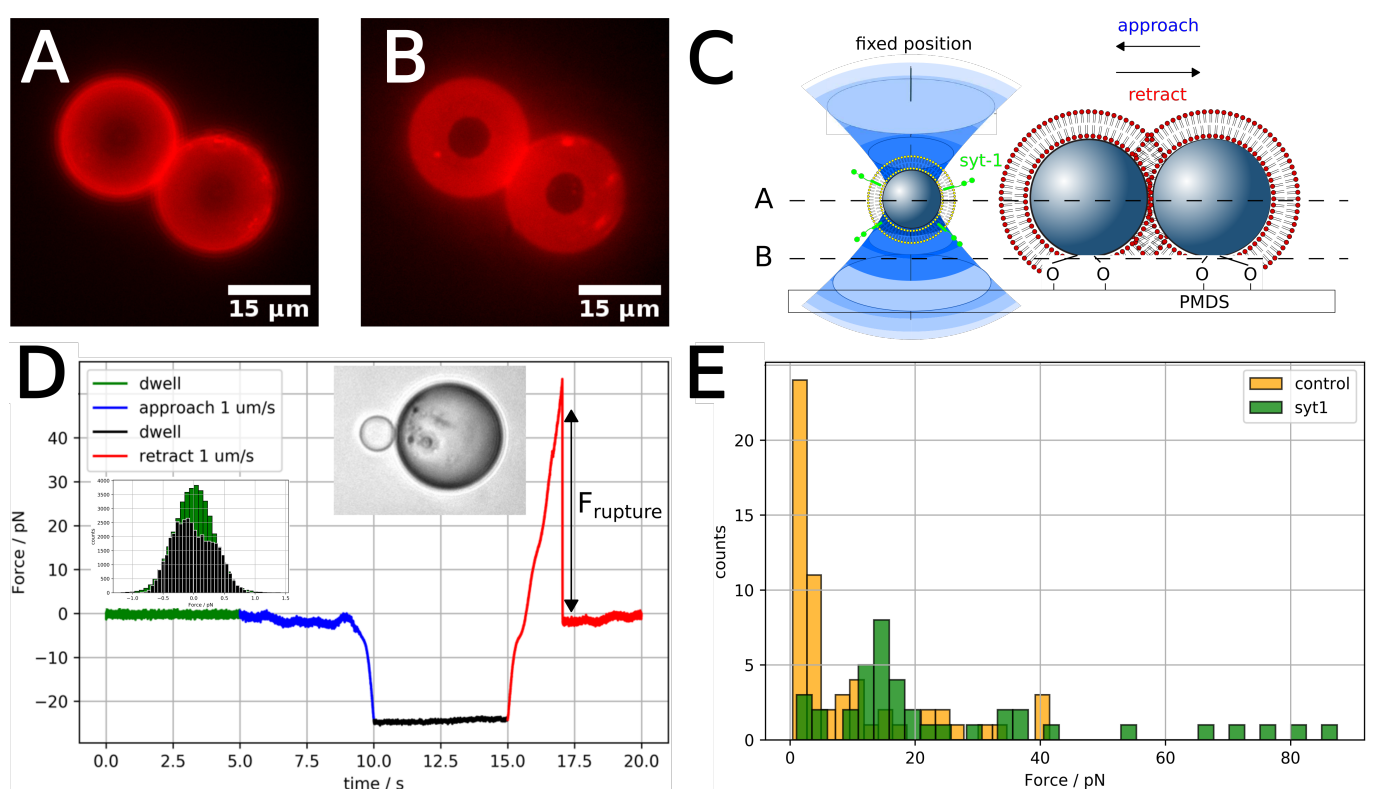

Figure 6.8: Binding properties and rupture forces between synaptotagmin and $\mathrm{PI}(4,5) \mathrm{P}_{2}$ - containing membranes. A-B: Fluorescence images of membrane-coated beads with a diameter of $15 \mu \mathrm{m}$ acting as the target membrane (membrane dye Texas Red). Focal plane at the bead center (A) and at the bottom (B) are shown. C: Schematic illustration of the experimental approach. One membrane coated bead with diameter of $4.75 \mu \mathrm{m}$ and reconstituted full-length synaptotagmin-1 (syt-1) is trapped within a focused laser beam and kept at a constant position. A piezo controlled stage moves the support with nanometer precision allowing to record approach and retraction curves, respectively. D: Representative force-time trace of single rupture events due to separation of Syt- 1 and $\mathrm{PI}(4,5) \mathrm{P}_{2}$. The experiment is split into four time-segments of 5 seconds duration each (color coded). 1: dwell used for calibration and force displacement relation (green). 2: approach phase with $1 \mu \mathrm{m} / \mathrm{s}$ speed where contact between the opposing membranes gets established (blue). 3: Application of a constant compression force $(22 \mathrm{pN})$ (black) 4: retraction phase with $1 \mu \mathrm{m} / \mathrm{s}$ speed (red). Successful binding is confirmed by an appreciable adhesion force dragging the trapped bead in the opposite direction. E: Distribution of rupture forces.

\subsubsection{Holographic Video Particle Tracking measurements}

This subsection introduces an approach where we add membrane coated beads free in solution and monitor their interaction with a solid-supported membrane on glass substrate. This method is a force-free approach to study membrane fusion and the influence of a calcium sensor by tracking the probe with HVPT and its capability to tether multiple beads to the opposing target membrane by membrane-protein interaction. The primary goal was to investigate the impact of $\mathrm{PI}(4,5) \mathrm{P}_{2}$ and synaptotagmin- 1 (syt- 1 ) on 
SNARE-mediated fusion in vitro. We performed two kinds of experiments, as shown in Figure 6.10. Firstly (Fig. 6.10, B) we tracked membrane coated beads consisting of DOPC:DOPE:DOPS:Chol:Dye-(Atto488 or TR)-DHPE (mol\%, ratio 55:22:11:11:1) doped with syb-1-116 and $\Delta \mathrm{N}$, respectively $(\mathrm{p} / \mathrm{l}=1: 500)$ and analyzed the trajectories with the particle motion's time-resolved MSDs after PCA and segmentation with PELT. Similar to the obtained results from optical tweezer measurements, we measured a decreased particle motion upon the progression from docking to fusion through merging of the lipid bilayers. Confined motion radii decreased from $r_{\text {conf, free }}>250 \mathrm{~nm}$ to $r_{\text {conf, contact }} \approx 50-250 \mathrm{~nm}, r_{\text {conf, docking }} \approx 15-45 \mathrm{~nm}$ and $r_{\text {conf, fusion }}<15 \mathrm{~nm}$, and we allocated them to fusion intermediates respectively (Fig. 6.9].

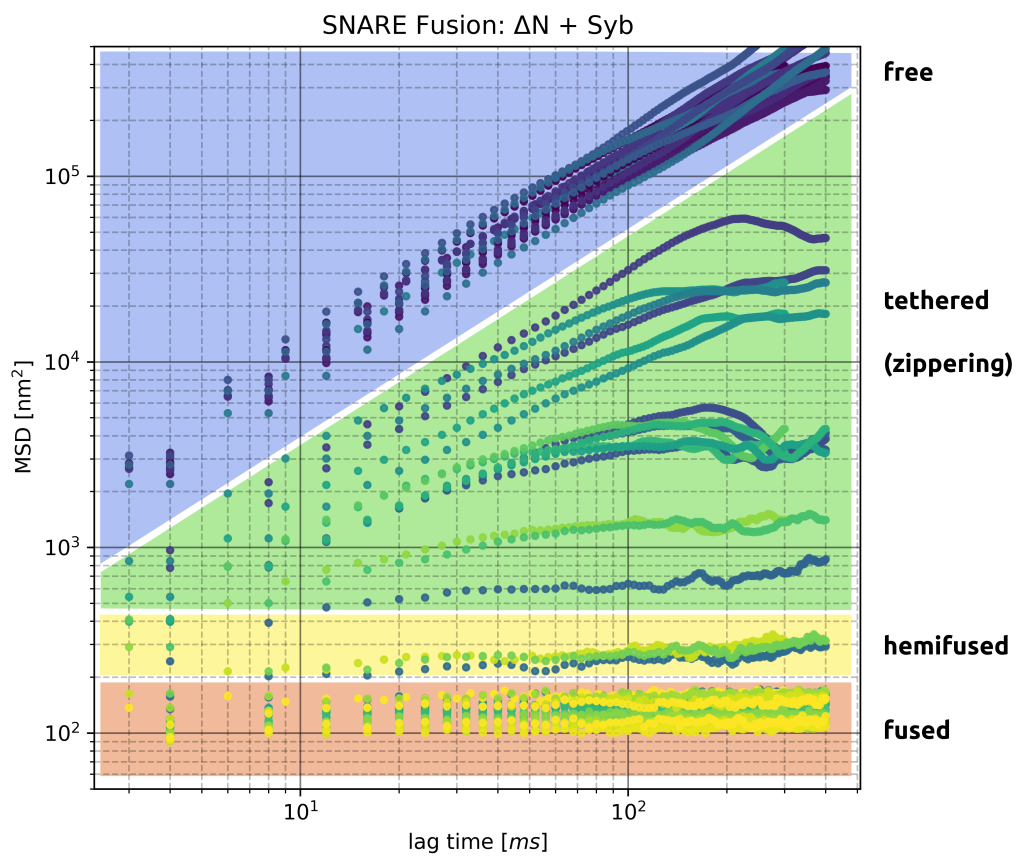

Figure 6.9: Mean square displacements of tracked membrane coated beads consisting of DOPC:DOPE:DOPS:Chol:Dye-(Atto488 or TR)-DHPE (mol\%, ratio 55:22:11:11:1) doped with syb-1-116 and $\Delta \mathrm{N}$, respectively $(\mathrm{p} / \mathrm{l}=1: 500)$. The particle motion's timeresolved MSDs after PCA and segmented with PELT. Confined motion radii decreased from $r_{\text {conf, free }}>250 \mathrm{~nm}$ to $r_{\text {conf, contact }} \approx 50-250 \mathrm{~nm}, r_{\text {conf, docking }} \approx 15-45 \mathrm{~nm}$ and $r_{\text {conf, fusion }}<15 \mathrm{~nm}$ color coded as a function of time.

The second approach (Fig. 6.10, C) was performed as a combination of Syt-1 mediated tethering and SNARE mediated fusion. Syt-1 tethering was investigated in the presence and the absence of the protein and additional calcium ions as a function of five different bilayers containing anionic lipids (PS) and different amounts of PI(4,5) $\mathrm{P}_{2}$; see Figure 3.9. Specifically, we deposited DOPC:POPE:Chol:TR-PE (50:29:20:1) membranes with reconstituted Syt- 1 on our microparticle against five separate target membranes on glass support to research the impact of PS and $\mathrm{PI}(4,5) \mathrm{P}_{2}$ on mem- 
brane recognition and also membrane fusion in the presence of SNAREs. We used DOPC:POPE:POPS:Chol:PIP $(4,5)_{2}$ :Atto488-DPPE varying with PS and PI(4,5) $\mathrm{P}_{2}$ concentration $\mathrm{N}$ (50:29:0:20:0:1, 50:19:10:20:0:1, 50-N:19:10:20:N:1 N=1\%, 2\%, 5\%) as the opposing target membrane (planar solid supported lipid bilayer). Via observing the particles' motion, we detect numerous confinement radii $\left(r_{\text {conf }}\right)$ and altered diffusive behavior over time when comparing different segments and their MSDs from the particles motion on different bilayers. We note that an increasing negative charge of the target membranes contributes to narrower radii of containment and closer interaction with the opposing membrane without membrane fusion involvement, as shown and discussed in the following section.

We reconstruct the membrane fusion landscape from all these measurements, starting with membrane-membrane and membrane-protein interaction till resolution into full fusion. As a marker of these states, we use measured confinement radii, as displayed in Figure 6.11.

For each confinement radius we fit a gaussian distribution to the bead's movement from its displacement and centered it respective to the radius of confinement $\Delta x+r_{\text {conf }}$ (see Supporting Figures 6.13 S1 and 6.14 S2). From that we obtain a reaction landscape during progression of time, which reflects all probe-substrate interactions. Figure 6.11 (C)-(G) schematically presents these interactions. Possible interactions between probe and substrate are: $C$ : Free movement without the interaction with a substrate under Brownian motion (free). D: Probe attaches to the target surface through electrostatic interaction and moves in a confined motion (EI: electrostatic interaction). E: Probe attaches to the surface through a Syt- 1 tether (syt) with $r_{\text {conf }}$, Syt $<r_{\text {conf }}$, EI. F: decreased confinement radius in which SNARE docking can happen (SNARE docking); $r_{\text {conf }}$, docking $<r_{\text {conf }}$, Syt. G: Radii where membrane fusion occurs (fusion) $r_{\text {conf }}$, fusion $<$ $11 \mathrm{~nm}$. We performed experiments on four independent repetitions for each bilayer to reconstruct these landscapes and accumulated 30 beads for each experiment. Results are represented as landscapes with a median trajectory, as shown in supporting Figure $6.15 \mathrm{~S} 3$, and will be discussed in the following section. 

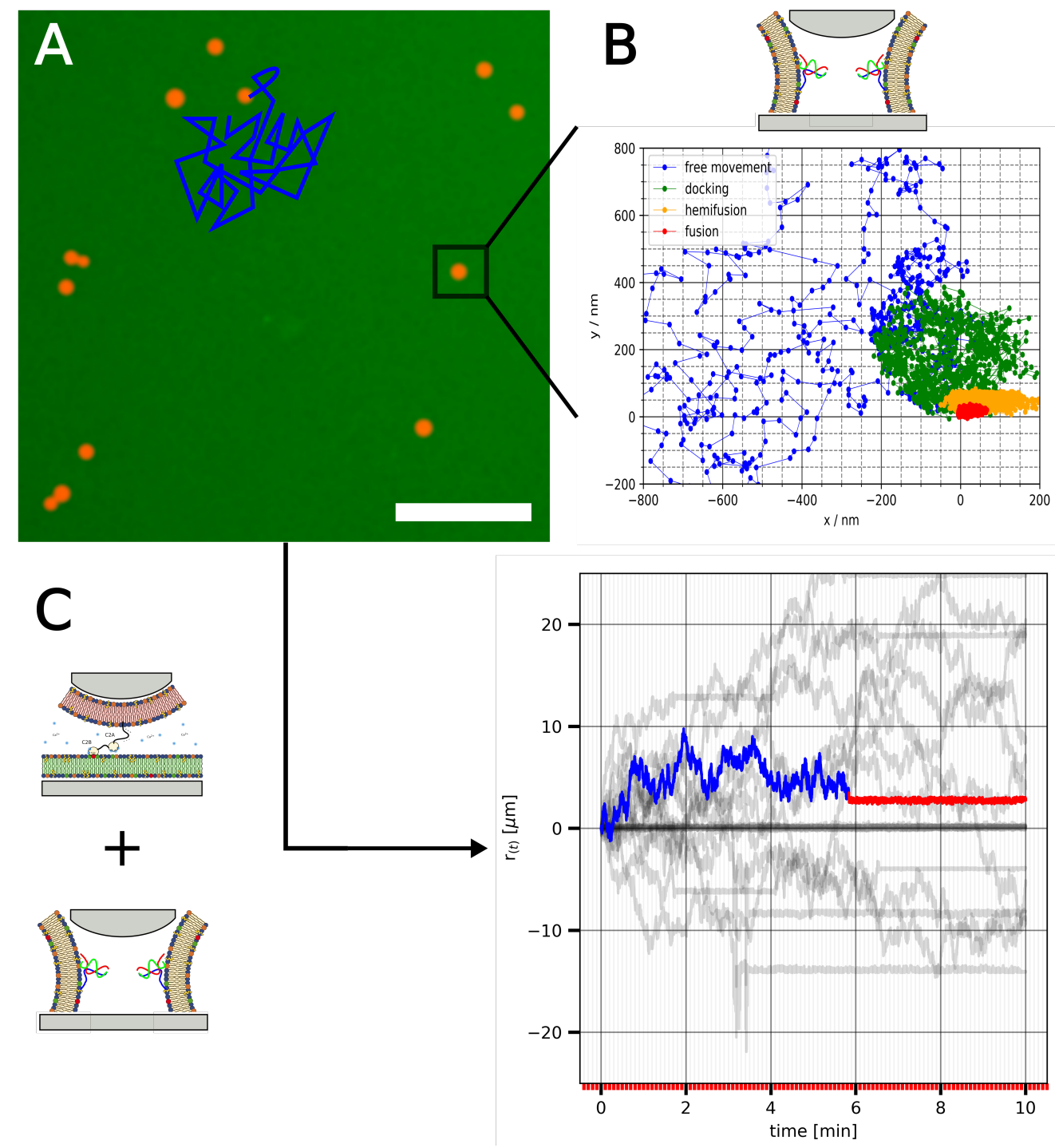

Figure 6.10: Experimental overview of HVPT colloidal probe assay to realize membrane interactions (C) or membrane fusion (B) and its combination. A: Dual-channel fluorescence image of a region of interest in which particle trajectories are obtained with HVPT. B: Single representative trajectory with of SNARE mediated membrane fusion resolve with 200 Frames per second. free particle diffussion, blue; docking, green; hemifusion, orange; full fusion, red. Membrane consisting of DOPC:DOPE:DOPS:Chol:Dye(Atto488 or TR)-DHPE (mol\%, ratio 55:22:11:11:1) doped with syb-1-116 and $\Delta \mathrm{N}$, respectively $(\mathrm{p} / 1=1: 500)$. C: Superposition of multiple trajectories of time-resolved coreconstitution experiment of Syt-1 $(\mathrm{p} / 1=1: 1000)$ and syb-1-116 $(\mathrm{p} / 1=1: 500)$ on a membrane coated probe (DOPC:POPE:Chol:TexasRed-DPPE: 50:29:20:1) against $\Delta \mathrm{N}(\mathrm{p} / 1=$ 1:500) on the target membrane side (DOPC:POPE:POPS:Cholesterol:PI(4,5) $\mathrm{P}_{2}$ :Atto488DPPE: 45:19:10:20:2:1). Trajectories of the control experiments without SNAREs are shown. Experiments are performed over a time course of 10 minutes with a resolution of 60 frames per second. After a particular time, membrane coated beads doped with Syt-1 attach to the $\mathrm{PI}(4,5) \mathrm{P}_{2}$ in the solid support membrane while its motion changes from free diffusive (blue) to a confined motion (red). 


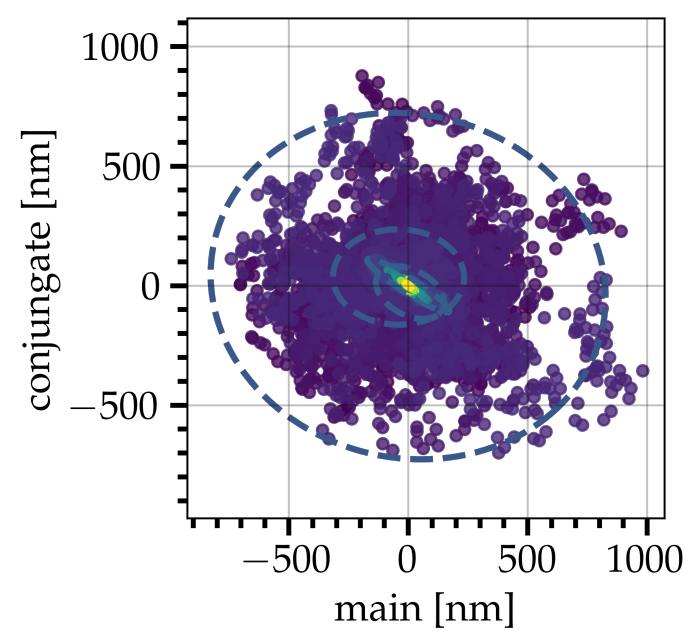

(a) smoothed trajectory + confinement radii

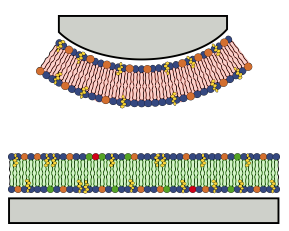

(c)

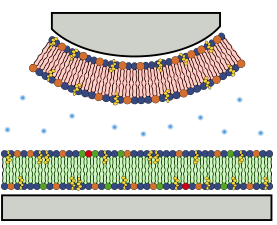

(d)

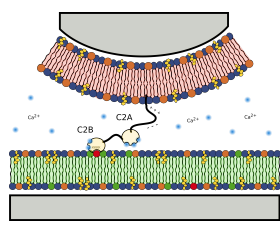

(e)

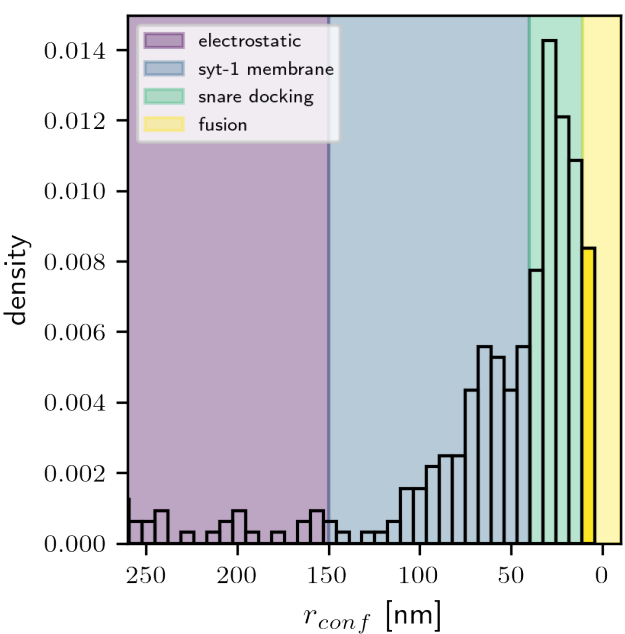

(b) $r_{\text {conf }}$ Distribution

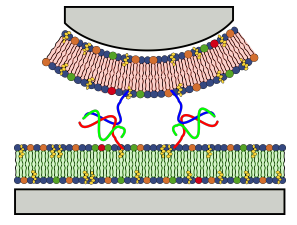

(f)

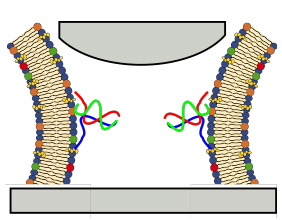

(g)

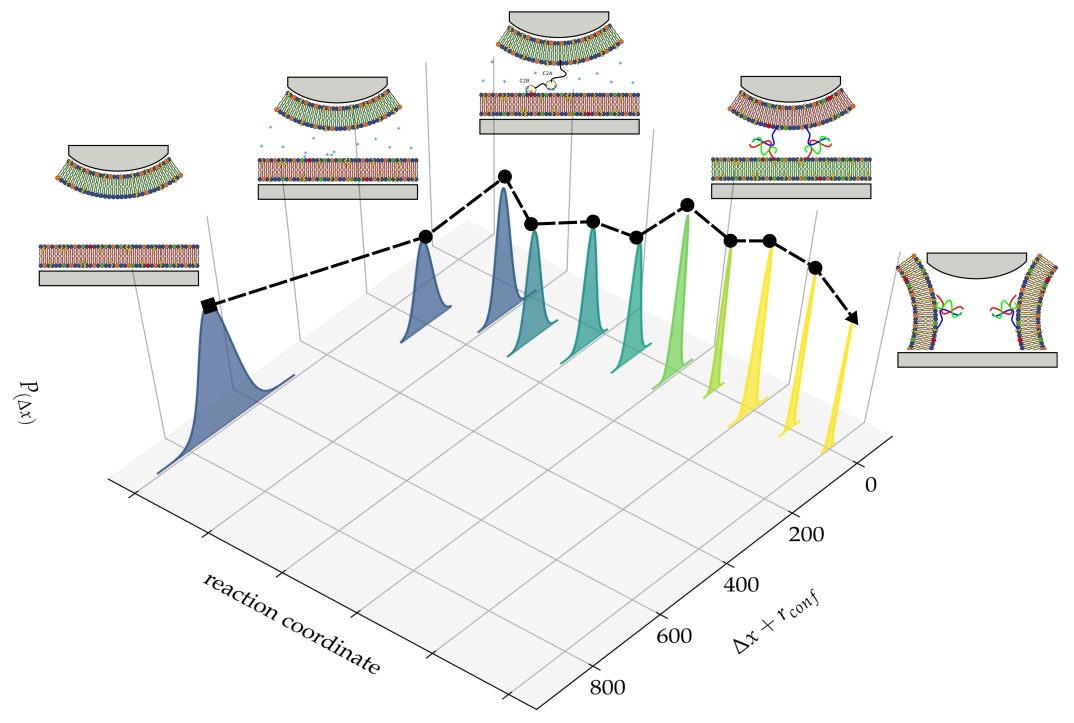

(h) landscape

Figure 6.11: Time-resolved landscape reconstruction of co-reconstitution fusion experiments with Syt- $1(\mathrm{p} / 1=1: 1000)$ and syb-1-116 $(\mathrm{p} / 1=1: 500)$ on a membrane coated probe (DOPC:POPE:Chol:TR-DPPE; 50:29:20:1) against $\Delta \mathrm{N}(\mathrm{p} / 1=1: 500)$ on the target membrane side (DOPC:POPE:POPS:Cholesterol:PI(4,5) $\mathrm{P}_{2}$ :Atto488-DPPE; 45:19:10:20:2:1). Schematic interactions: (c) free movement, (d) electrostatic interaction, (e) Syt-1 tethering and confined motion, (f) SNARE docking and (g) membrane fusion. 


\section{Supporting Figures:}
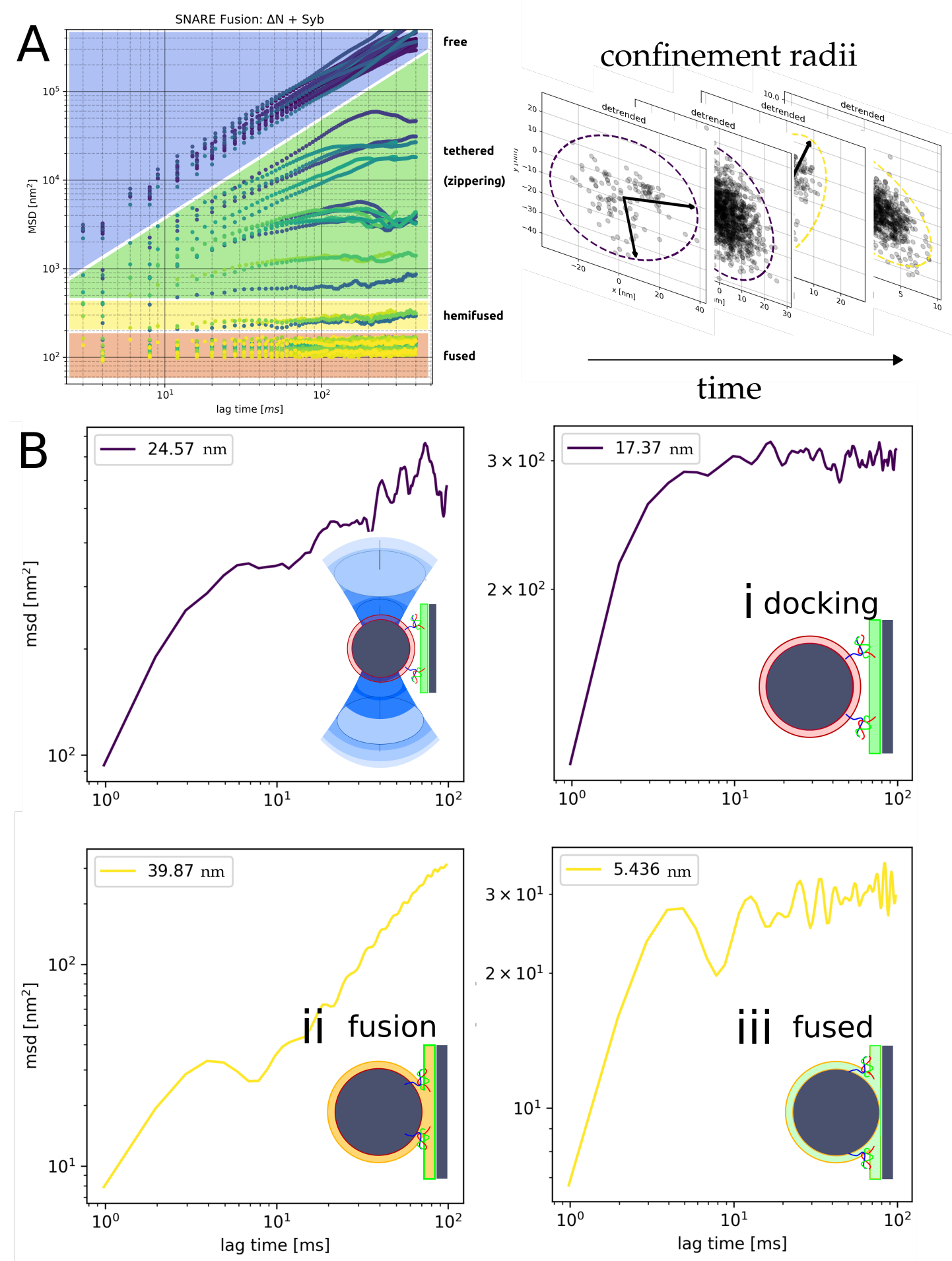

Figure 6.12: S0: PCA- and MSD-Analysis of optical tweezer data with confinement radii otbtained from fitted data before and after fusion. $1 \mu \mathrm{m}$ membrane-covered bead comes into contact with a $15 \mu \mathrm{m}$ membrane-coated bead by OT. Membrane consists of DOPC:DOPE:DOPS:Chol: Oregon-Green-(/-TR)-DHPE (mol\%, ratio 55:22:11:11:1). States from trajectories are analysed by MSD as function of time. 3 different confinement radii are observed: (i) $24-30 \mathrm{~nm}$ (ii) $11-39 \mathrm{~nm}$ (iii) $4.5-5.4 \mathrm{~nm}$. Note: Confinement radii of state (ii) are shifted due to an active process. In this context docking and merging of bilayers. 

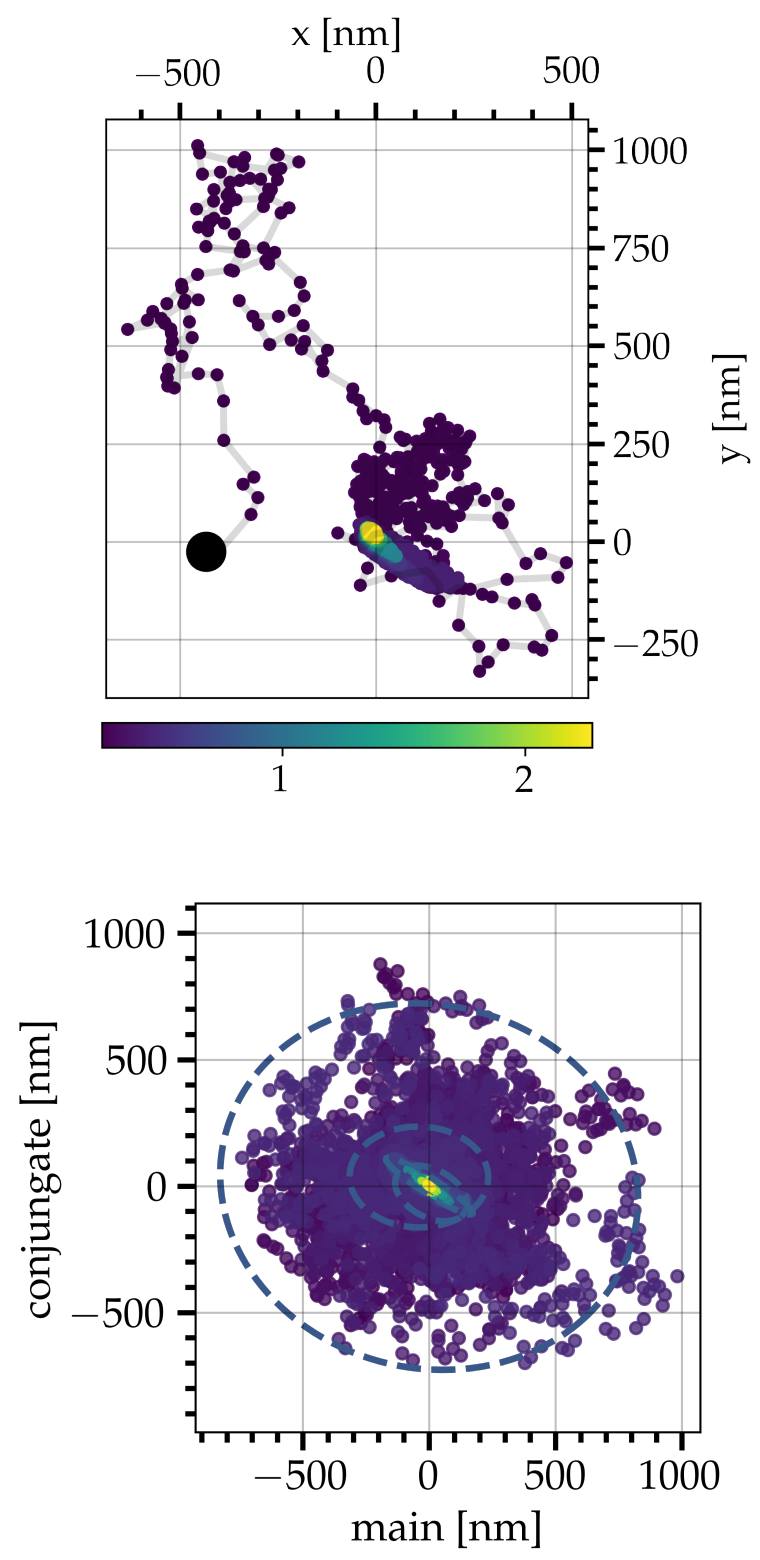

Figure 6.13: S1: Representative trajectory from HVPT fusion experiments with Syt-1 $(\mathrm{p} / 1=1: 1000)$ and syb-1-116 $(\mathrm{p} / 1=1: 500)$ on a membrane coated probe (DOPC:POPE:Cholesterol:TR-DPPE; 50:29:20:1) against $\Delta \mathrm{N}(\mathrm{p} / 1=1: 500)$ on the target membrane side (DOPC:POPE:POPS:Chol:PI(4,5)P ${ }_{2}$ :Atto488-DPPE: 45:19:10:20:2:1). Time-resolved and color coded confinement radii and motion data over a time course of 2 minutes, recorded with 200 frames per second. 

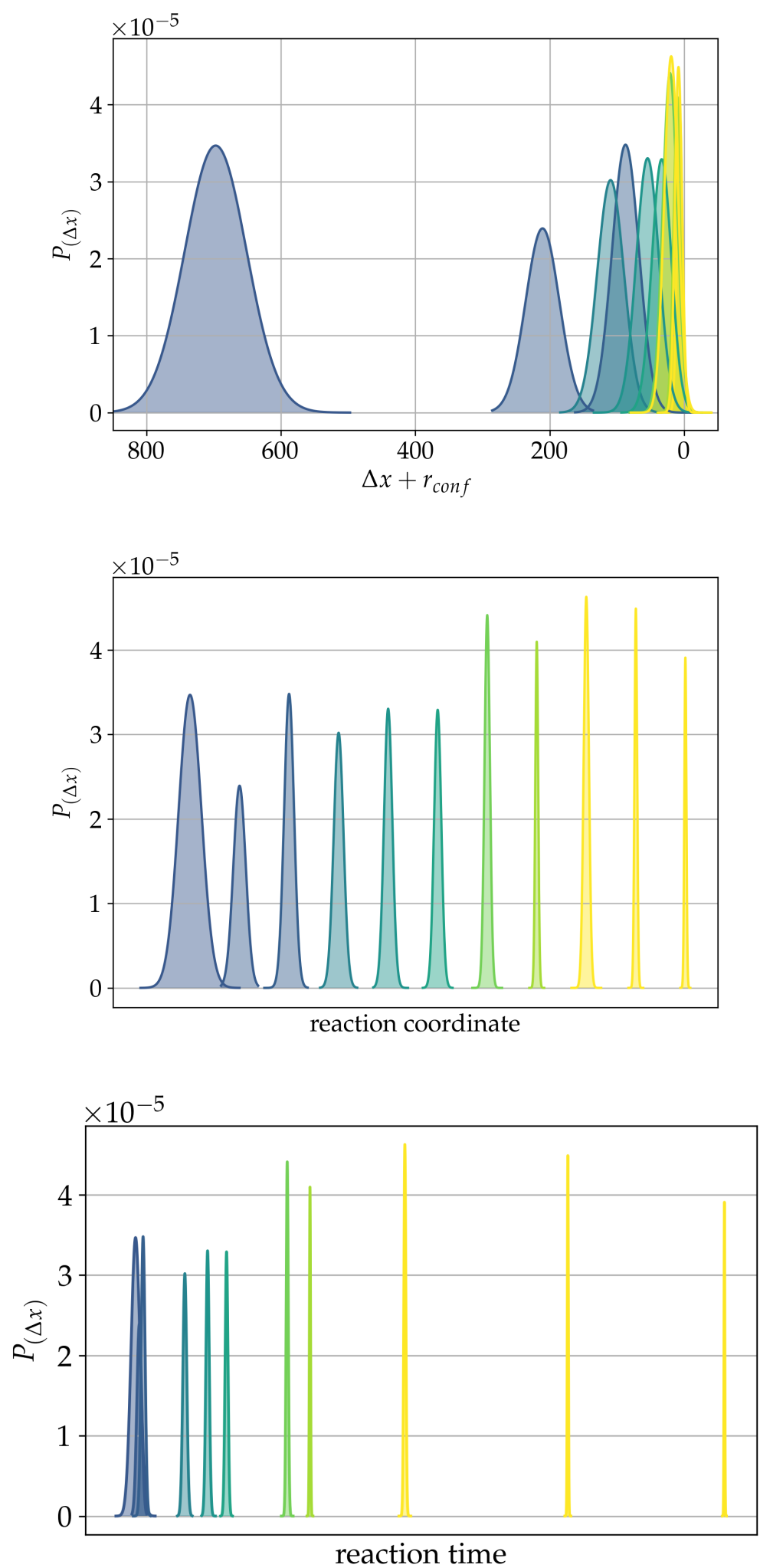

Figure 6.14: S2: Basis of landscape reconstruction: Gaussian distributions obtained from motion data and related to its confinement radius. Time-resolved and color coded confinement radii and motion data over a time course of 2 minutes, recorded with 200 frames per second. 

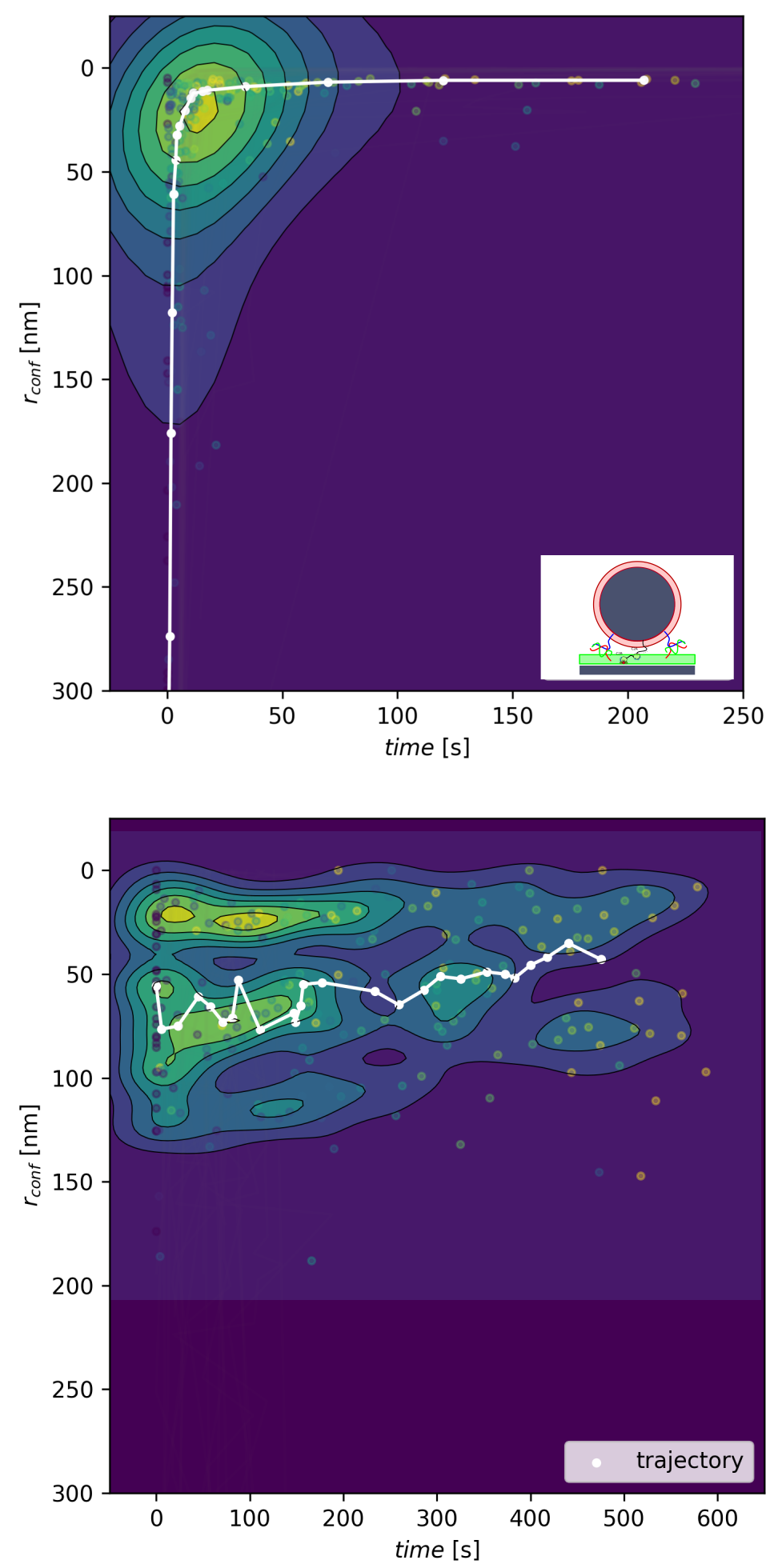

Figure 6.15: S3: Landscape reconstruction: KDE overlayed scatterplots of membrane coated bead trajectories (DOPC:POPE:Chol:TR-DPPE after contact to SSM (DOPC:POPE:POPS:Chol:PI(4,5) $\mathrm{P}_{2}$ :Atto488-DPPE : (48:19:10:20:2:1)) recorded with HVPT in the presence of SNAREs and Syt-1 (top) and in the absence of SNAREs while Syt-1 is present (bottom). Derived is a median trajectory from the confinement radius of the beads movement over a period of $600 \mathrm{~s}$; shown in white. 


\subsection{Influence of $\mathrm{PI}(4,5) \mathrm{P}_{2}$ for calcium-mediated and synaptotagmin- triggered exocytosis}

The mainframe of neuronal signal transmission in a chemical synapse is the $\mathrm{Ca}^{2+}$ -dependent synchronous secretion of neurotransmitter-loaded synaptic vesicles on a sub-millisecond time scale [23, 39]. To ensure this highly controlled synchronous process with $\mathrm{Ca}^{2+}$-influx, a sensitive $\mathrm{Ca}^{2+}$ sensor is required [2, 18]. Syt-1 is a major candidate for a neuronal exocytosis sensor of $\mathrm{Ca}^{2+}$, but the exact mechanism of how Syt-1 triggers $\mathrm{Ca}^{2+}$-evoked neurotransmitter release remains unclear [15, 34]. For this reason, to fully understand the mode of action of Syt-1, the study of the impact of Syt-1 on SNARE-mediated membrane fusion under well-defined in vitro conditions is of significant importance. It is well known that synaptic vesicle exocytosis is caused by $\mathrm{Ca}^{2+}$. The discovery of the fusion reaction regulating sensor $\mathrm{Ca}^{2+}$ unlocked the way to tracing this transduction pathway, offering a way to illustrate the controlled exocytosis mechanism [37]. In neurons and neuroendocrine cells, a 65-kDa protein, Syt-1, localized to synaptic vesicles, was identified [17].

A single membrane-spanning domain, a short intraluminal domain, and a broad cytoplasmic domain consisting of tandem $\mathrm{C} 2$ domains, $\mathrm{C} 2 \mathrm{~A}$ and $\mathrm{C} 2 \mathrm{~B}$, connected by a linker is revealed by the primary Syt-1 sequence [38]. It is suspected that this protein binds directly to phosphatidylinositol-4,5-bis-phosphate $\left(\mathrm{PI}(4,5) \mathrm{P}_{2}\right)$, which makes up more than $80 \%$ of the total surface area of the microdomains of syx-1A. $\operatorname{PI}(4,5) \mathrm{P}_{2}$ was found to directly connect the $\mathrm{C} 2 \mathrm{~B}$ domain of Syt- 1 and syx-1A in a $\mathrm{Ca}^{2+}$-independent manner based on the PI $(4,5) \mathrm{P}_{2}$ polyanion headgroup and the syx-1A (260-KARRKK-265) and C2BB syx-1A polybasic sequences (324-KKKK-327) [7, 24, 28, 30, 31, 38].

In this part, we determine the effect of $\mathrm{PI}(4,5) \mathrm{P}_{2}$ on the efficiency of membrane fusion and the lifetime of the different intermediates. For this reason, and to mimic the situation of the synaptic vesicle, the supported bilayers were composed with $\mathrm{PI}(4,5) \mathrm{P}_{2}$ and PS. We use a reconstituted system composed of SNARE proteins and Syt-1 and allow $\mathrm{Ca}^{2+}(100 \mu \mathrm{M})$ to cause fusion. In executing so, we refer to using two methods that use colloidal particles as a probe - initially, AFM-controlled investigations of the strength of adhesion between a $15 \mu \mathrm{m}$ membrane coated bead and planar solidsupport support, mediated by synaptotagmin are conducted. Then experiments to track membrane protein interactions before and during fusion using holographic video particle tracking of membrane coated beads with a size of $1 \mu \mathrm{m}$ in diameter are performed. 


\subsubsection{Atomic Force Spectroscopy - membrane interaction and forces of syn- aptotagmin}

We use POPC:Bodipy (99:1) with reconstituted Syt-1 (p/1 = 1:100) on our colloidal probe (AFM-CP-Cantilever) against a target membrane on glass support to study the adhesive interaction properties of Syt- 1 to an opposite membrane targeting $\mathrm{PI}(4,5) \mathrm{P}_{2^{-}}$ interaction DOPC:DOPS:PIP(4,5) 2 :TR (87:11:1:1) under buffer condition containing $\mathrm{Ca}^{2+}$-Ions. By analyzing AFM retraction curves, we obtain rupture forces representing different interaction modes between Syt- 1 and the target membrane according to its distance from the surface.

Force-distance measurements were carried out with an MFP3D (Asylum Research, Santa Barbara, CA, USA). The spring constants of the cantilevers were calibrated using the thermal noise method and were found to be in a range of 5-20 pN/nm. All force-distance cycles were operated with an approach velocity of $500 \mathrm{~nm} / \mathrm{s}$, a retraction velocity of $500 \mathrm{~nm} / \mathrm{s}$, a contact time of $10 \mathrm{~s}$ and $200 \mathrm{pN}$ loading force. The measurements were performed in HP150 buffer or in HP150-Ca buffer (20 mM HEPES, $150 \mathrm{mM} \mathrm{KCl}$, $\left.1 \mathrm{mM} \mathrm{CaCl}_{2}, \mathrm{pH} 7.4\right)$, respectively. For each set of parameters at least 200 force-distance cycles were performed ${ }^{1}$

It is assumed that, by a small water film, the membranes bind to the substrates and can thus be seen as rigid surfaces that can not be stripped off from the surface. It can be stated only by this presumption that the measured peaks are correlations with the protein or membrane and not the membrane's elasticity or other properties. While the membrane- and protein-functionalized spherical cantilever was in contact with the surface, an interaction between the two membranes has taken place, which has to be separated again. When the cantilever is removed from the surface, interactions or bonds between molecules can be broken by an applied force. This process is used in the force measurements of atomic force microscopy so that the interactions associated with synaptotagmin can be obtained in this experiment. Weak interactions are separated immediately. For strong interactions, it is likely that the protein through which the interaction exists initially unfolds, before finally, the interaction is softened by changing the conformation of the protein. The last possibility is pulling out tethers of the membrane if the interactions are stronger than the membrane's cohesion. The weakest interaction between the two membranes is always the bond that is ruptured. The measurements shown in Figure 6.16 reveal a complex interplay between synaptotagmin and the target membrane. In the following we discuss possible binding interactions between $\mathrm{C} 2 \mathrm{~B}$ and $\mathrm{PIP}_{2}, \mathrm{C} 2 \mathrm{~A} / \mathrm{C} 2 \mathrm{~B}$ and PS, and their combination:

\footnotetext{
${ }^{1}$ Experiments were planned and conducted by Marieelen Oelkers [29].
} 
Synaptotagmin's C2B domain can bind to $\mathrm{PIP}_{2}$ independently of calcium because of a region with high lysine density in the protein. Considering only $\mathrm{PIP}_{2}$ and no DOPS in the substrate membrane, only the $\mathrm{C} 2 \mathrm{~B}$ domain can interact with the membrane. $\mathrm{C} 2 \mathrm{~A}$ remains without interaction with the surface and only serves as a linker between $\mathrm{C} 2 \mathrm{~B}$ and the transmembrane domain. If the protein is now pulled away from the surface, tension will probably initially act only on the $\mathrm{C} 2 \mathrm{~A}$ domain. If the bond between $\mathrm{C} 2 \mathrm{~B}$ and $\mathrm{PIP}_{2}$ is weak, it would likely rupture if the force applied was low. However, if the interaction is stronger, there may be a chance that the C2A domain will unfold due to the force acting on it.

When negatively charged lipids and no $\mathrm{PIP}_{2}$ are present in the membrane, the $\mathrm{C} 2 \mathrm{~B}$ domain can interact with two binding sites via calcium ions with PS. The C2A domain has three binding sites for calcium-dependent binding with PS. Thus the binding between synaptotagmin and the membrane in a calcium environment is exerted by both domains. Without calcium, there should be no interaction with synaptotagmin with this membrane composition.

With both $\mathrm{PIP}_{2}$ and DOPS present in the membrane, all of these bonds can exist simultaneously in a calcium environment. Multiple ways in which the interactions are released when the cantilever is pulled off from the surface are possible. Since the C2B domain is connected to both $\mathrm{PIP}_{2}$ and PS, it can be assumed that it is more strongly bound to the membrane than only with one of the two bonds. If no calcium existed in the surrounding buffer with this membrane composition, the interactions would presumably behave like those with only $\mathrm{PIP}_{2}$ in the bilayer. Perhaps more unspecific interactions would exist due to the charged lipids.

Most importantly, it proves that detecting and partially identifying the individual interactions between synaptotagmin and the $\mathrm{PIP}_{2} / \mathrm{PS}$-containing membrane is possible. Thus, calcium-dependent membrane-membrane interactions suddenly reach the range of forces from the interaction between the $\mathrm{C} 2 \mathrm{~A}$ domain and the membrane, making this exact association impracticable. The interaction of the $\mathrm{C} 2 \mathrm{~A}$ domain is of different strength since it can rise from several cooperatively connected binding pockets. The possible variations of the $\mathrm{C} 2 \mathrm{~B}$ domain interaction are only a few nanometers apart so that they likewise cannot be separated from one another in a calcium environment.

Nonetheless, we observed that the C2A domain can unfold by pulling it apart, while the $\mathrm{C} 2 \mathrm{~B}$ domain is still bound to the membrane.

The comparison between $\mathrm{PIP}_{2}$-free and $\mathrm{PIP}_{2}$-containing bilayer in calcium containing environment, as shown in Figure 6.16, displays the following as a characteristic fin- 
gerprint of interactions when synaptotagmin and $\mathrm{PIP}_{2}$ are present. In Figure 6.16 an experiment is shown with PS and $\mathrm{PIP}_{2}$ in the substrate membrane without synaptotag$\min$ in the probe membrane at a retraction speed of $500 \mathrm{~nm} / \mathrm{s}$, hardly any interaction was observed since only electrostatic membrane-membrane interactions were available. These forces occur approximately between $5-200 \mathrm{pN}$ and are essentially unspecific and randomly distributed over a range of position distances up to $100 \mathrm{~nm}$ away from the surface. On the other hand, the Syt- 1 containing measurements show a complex fingerprint of membrane-membrane and membrane-protein interaction. According to $\mathrm{C} 2 \mathrm{~A}$ unfolding and $\mathrm{C} 2 \mathrm{~B}$ rupture events, these are evidence of successful tethering and bridging of Syt-1 between the opposing membranes; most evidently to $\mathrm{PIP}_{2}$.

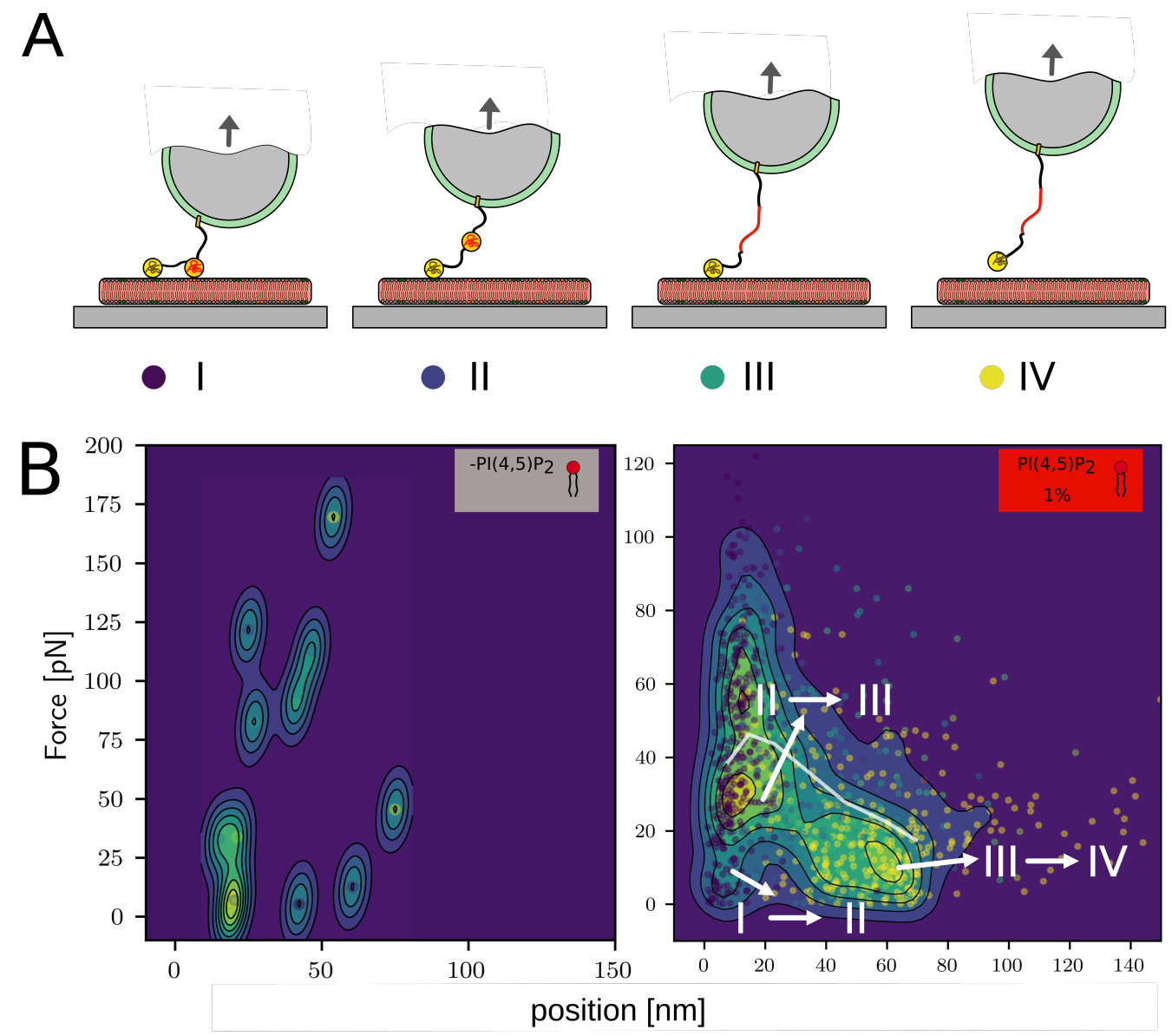

Figure 6.16: A: Schematic representation of AFM retraction sequence visualizing Syt-1 unfolding pathway and its ultimate detachment from the target membrane marked with yellow. I and II: Lifting of C2A domain. III: stretching and unfolding of C2A domain. IV. Rupture of the bond between $\mathrm{C} 2 \mathrm{~B}$ and $\mathrm{PIP}_{2}$. B Comparison of force-positionmap showing interactions between a colloidal probe, coated with a POPC membrane including Syt-1, and a planar PC membrane containing PIP $_{2}$ and PS in the absence (left) and with the presence of $\mathrm{PIP}_{2}$ (right) as calcium-Ions are in solution. Rupture forces are obtained from peaks of the force-distance curves' retraction path. Calcium concentration was $1 \mathrm{mM}$ and dwell time in contact was in between 3-10 s. 


\subsubsection{Interaction landscapes of synaptotagmin from tethered particle moti- on experiments and its implications on SNARE mediated membrane fusion}

In this part we discuss the interaction landscapes of synaptotagmin obtained from tethered particle motion experiments as introduced above for one single trajectory. Syt- 1 tethering was investigated in the presence and the absence of the protein and additional calcium ions as a function of five different bilayers containing anionic lipids (PS) and different amounts of $\mathrm{PI}(4,5) \mathrm{P}_{2}$. Specifically, we deposited DOPC:POPE:Chol:TR-PE (50:29:20:1) membranes with reconstituted Syt-1 on our microparticle against five separate target membranes on glass support to research the impact of PS and PI $(4,5) \mathrm{P}_{2}$ on recognition and also membrane fusion in the presence of SNAREs. We used DOPC:POPE:POPS:Chol:PIP $(4,5)_{2}$ :Atto488-DPPE varying with PS and PI(4,5) $\mathrm{P}_{2}$ concentration $\mathrm{N}$ (50:29:0:20:0:1, 50:19:10:20:0:1, 50-N:19:10:20:N:1 N=1\%, $2 \%, 5 \%$ ) as the opposing target membrane (Fig. 6.17, and Fig. 3.9). Further we look at the influence of synaptotagmin and calcium $(+100 \mu \mathrm{M})$ on the trajectories of SNARE mediated membrane fusion for a molar ratio of $\mathrm{PI}(4,5) \mathrm{P}_{2}$ at $\mathrm{N}=2 \%$ in the bilayer (DOPC:POPE:POPS:Chol:PIP(4,5)2:Atto488-DPPE, 50-N:19:10:20:N:1) (Fig. 6.18). For each construction, experiments were performed on a minimal of four different bilayer preparations and under the accumulation of at leat 40 single bead trajectories only bilayers with 95\% surface coverage and FRAP mobility were used. Landscape constructions can be found enlarged in the supporting figures (Fig: 6.22 -6.26 S:1-5)

First, we performed experiments without synaptotagmin as control. The interaction between the bead membrane and the planar membrane was lowest without the addition of PS. Most of the beads moved freely in solution and only they adhered to the surface of the membrane for a short period of time. This interaction was so weak that beads bound and re-bound. These beads moved in relatively weak confinement $r_{\text {conf }}>200 \mathrm{~nm}$. As soon as we introduced negative lipids into the target membrane, the electrostatic interactions between the two membranes were intensified so that the beads in smaller confinement radii would bind, and their mobility was, therefore, more restricted $r_{\text {conf }}<$ $200 \mathrm{~nm}$. For PI $(4,5) \mathrm{P}_{2}$ at $\mathrm{N}=2 \%$ we observe $r_{\text {conf }}<35-50 \mathrm{~nm}$. From this we conclude that the membrane surface charges and the surrounding ionic environment dictates whether and how strong membranes come into close contact. However, within this range of confinement radii, we don't observe lipid merging or evidence of membrane fusion via fluorescence microscopy. In literature, clustering and domain forming has also previously been the object of interest in many experiments, both computational and experimental, in structures involving charged lipids and ions [43]. For example, both in experiments and computer simulations, $\mathrm{PIP}_{2}$ clustering was observed, and 
the clustering behavior was found to rely on the existence of interacting membrane proteins. Furthermore, domain formation in PS/PC monolayers was found to be caused by $\mathrm{Ca}^{2+}$ in experiments and simulations [32]. Especially formation of nanodomains of clustered PIP 2 lipids was observed by Koldsø et al. [16].

Additional to this knowledge, we observe that an increasing negative charge of the target membranes on the planar support leads to substantially smaller confinement radii and, therefore, closer contact between the opposing membranes the presence of calcium ions strongly enhances this effect. Calcium affects mobility of bound beads to confinement values of $r_{\text {conf }} \approx 50 \mathrm{~nm}$ and below for increased negative charge. This observation conserves for experiments conducted in the presence and the absence of Syt-1 as well. A metastable bond between probe and membrane was observed in all protein-free experiments. Despite a small confinement radius and a significant reduction in mobility, bound or adhered beads could spontaneously detach from the surface.

When reconstituting Syt-1 into our membrane probe we observe confined particle motion with radii between the ones of pure bilayer experiments and electrostatic $\mathrm{Ca}^{2+}$ membrane bridging. $\mathrm{PI}(4,5) \mathrm{P}_{2}$ was found to specifically interact with Syt- 1 even in the absence of calcium ions, which indicates $\mathrm{PI}(4,5) \mathrm{P}_{2}$ to act as a guide for the $\mathrm{Ca}^{2+}$-sensor to sites of fusion, especially in molar concentration above $1 \%$. Despite some additional repulsion, Syt-1 stabilizes binding affinity towards the target membrane compared to the plain bilayers. Most of the beads adhere immediately to the opposite membrane surface so that immediate contact can be observed without the probe being pulled off by thermal fluctuations.

Again this effect is highly increased in the presence of $\mathrm{Ca}^{2+}$-Ions $r_{\text {conf }}<11-30 \mathrm{~nm}$. That is not surprising because the $\mathrm{Ca}^{2+}$ affinity of synaptotagmin is markedly increased by a specific interaction of its C2B domain with phosphatidylinositol [33, 41]. The addition of calcium ions ensures a similar overlap in all target membranes. Here the confinement radius is narrowed so that the bilayers are in a tightly docked state, but without membrane fusion.

We understand that it is precisely this close docking state, the high connection affinity, and the short membrane to membrane distance that will influence the reaction kinetics and the interplay between SNAREs and synaptotagmin.

For this reason, we carried out four further experiments in which we examined the influence of calcium and synaptotagmin on the lifetime between docking and membrane fusion triggered by SNAREs. The constructed fusion landscapes for these are shown in 
Figure 6.18. In these experiments, every contact between the two membranes lead to fusion and merging bilayers. As shown in Figure 6.1. membrane fusion is characterized by several steps. One can imagine that the merging and the contact between the two glass surfaces restrict mobility, particularly in this case to confinement radii smaller than $11 \mathrm{~nm}$. Over 100 seconds, all trajectories point to a confinement radius of $4 \mathrm{~nm}$. We show that this tethered particle motion's experimental approach reveals how Syt-1 aids tethering followed by syb1-116 $+\Delta \mathrm{N}$ induced membrane fusion. The critical factor is the time between docking and fusion. We extract this parameter by manual fitting with a sigmoidal function, as shown in Figure 6.21 S0. The obtained results will be summarized and discussed in the following part. 


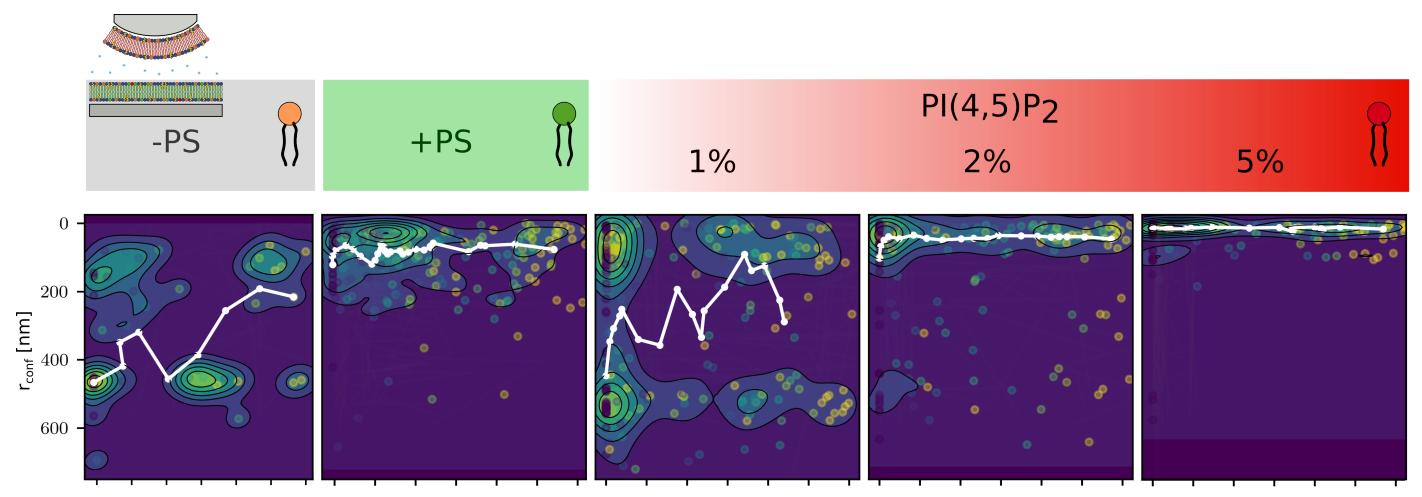

(a) Control without Syt-1 and without addition of calcium
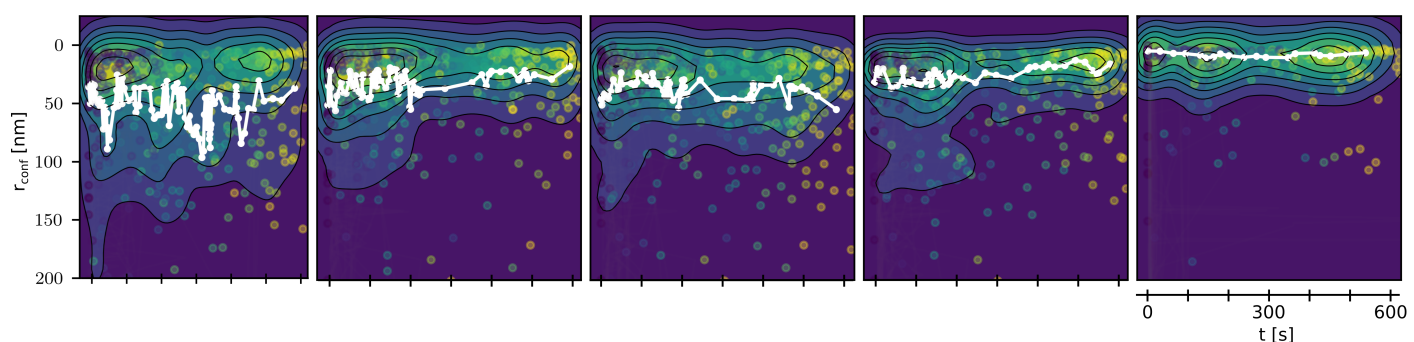

(b) Control without Syt-1 and addition of calcium

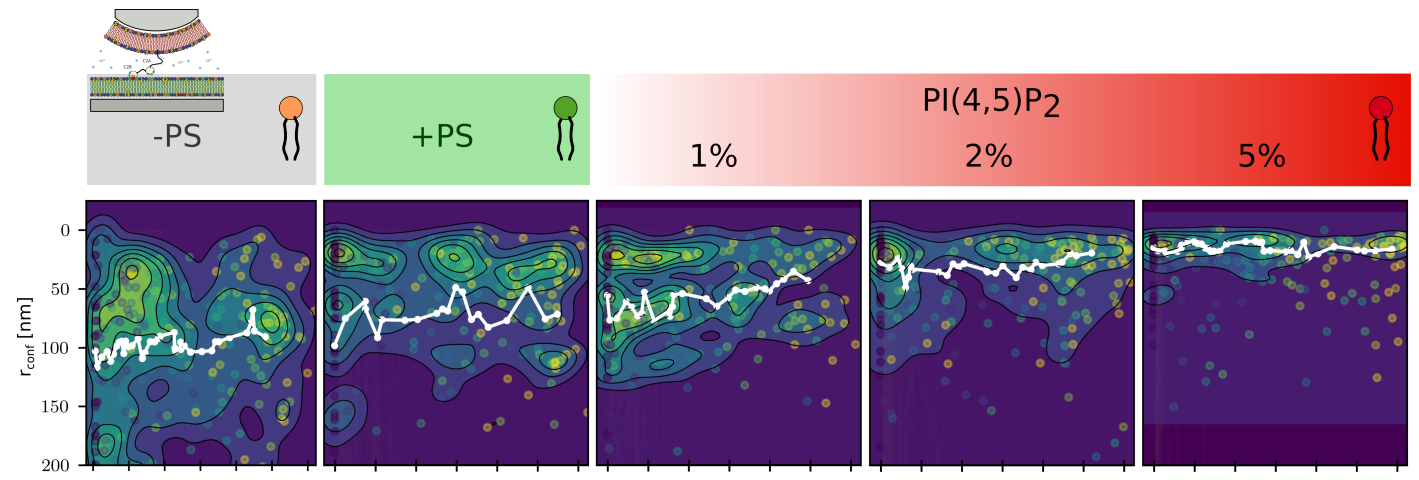

(c) Syt-1 without addition of calcium
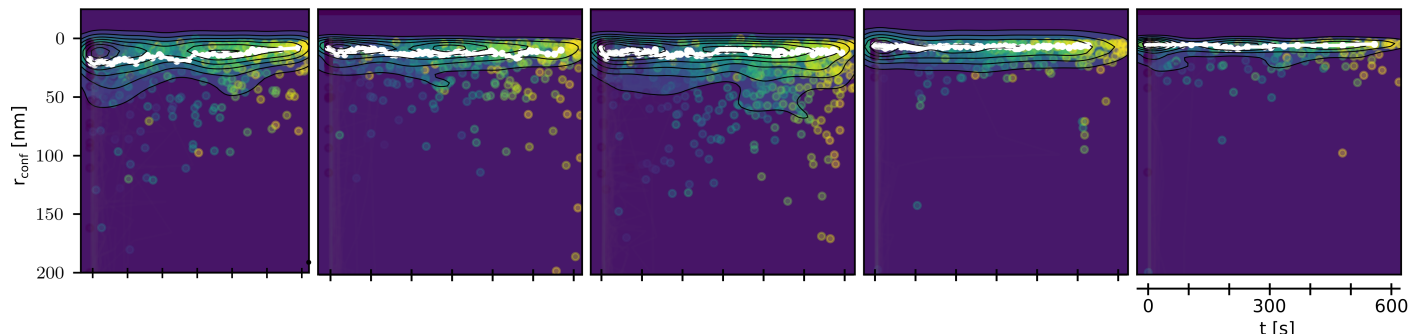

(d) Syt-1 with addition of calcium

Figure 6.17: Constructed interaction landscapes from TPM experiments in the presence and absence of f-Syt- 1 and calcium ions. Scatterplots of $r_{\text {conf }}$ as function of time from membrane coated bead trajectories (DOPC:POPE:Chol:TexasRed-DPPE either pure or doped with f-Syt-1: $\mathrm{p} / 1=1: 1000)$ after contact to SSM differing in PS and PIP $(4,5)_{2}$ molar ratios $n$ (DOPC:POPE:POPS:Chol:PI(4,5)P $\mathrm{P}_{2}$ :Atto488-DPPE: 50:29:0:20:0:1, 50:19:10:20:0:1, 50$n: 19: 10: 20: n: 1 n=1 \%, 2 \%, 5 \%)$ recorded with HVPT at $59.8 \mathrm{~Hz}$. Beads are monitored over a time period of $600 \mathrm{~s}$. Median trajectory is shown in white. 


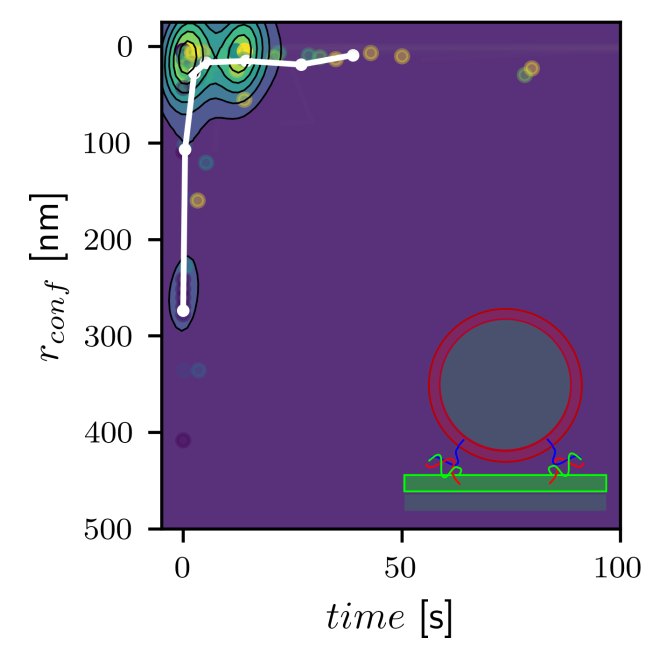

(a) SNAREs

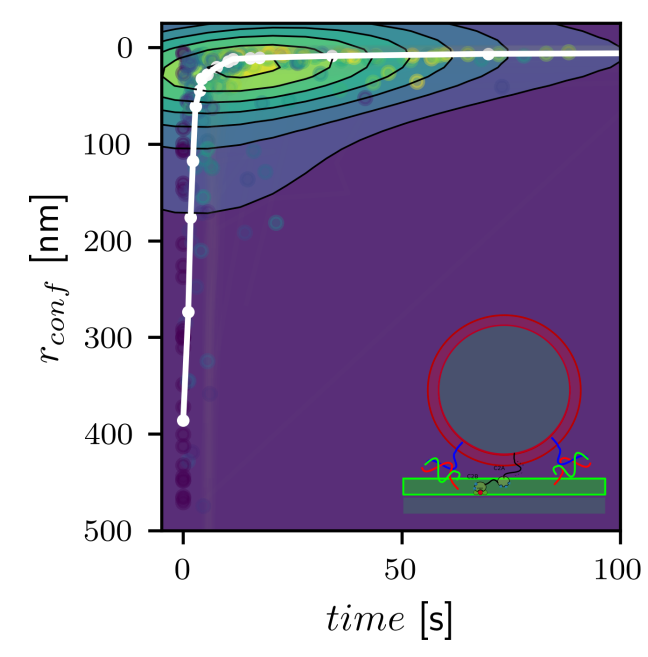

(c) Influence of Syt-1

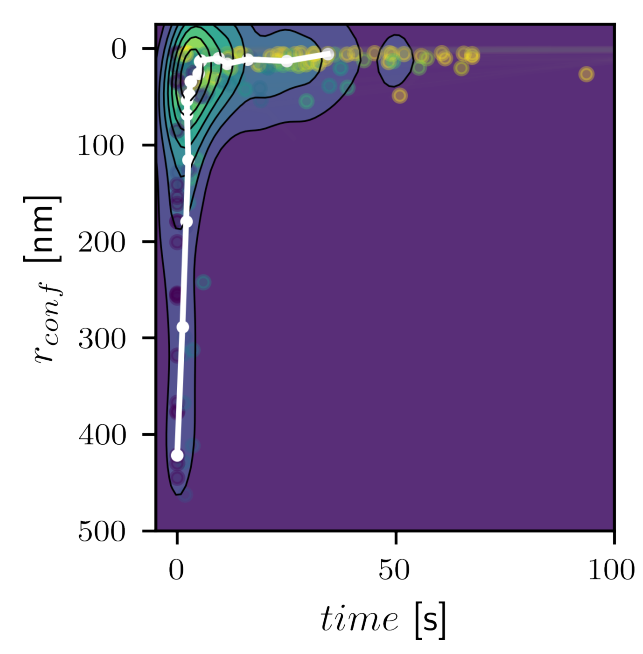

(b) Influence of calcium

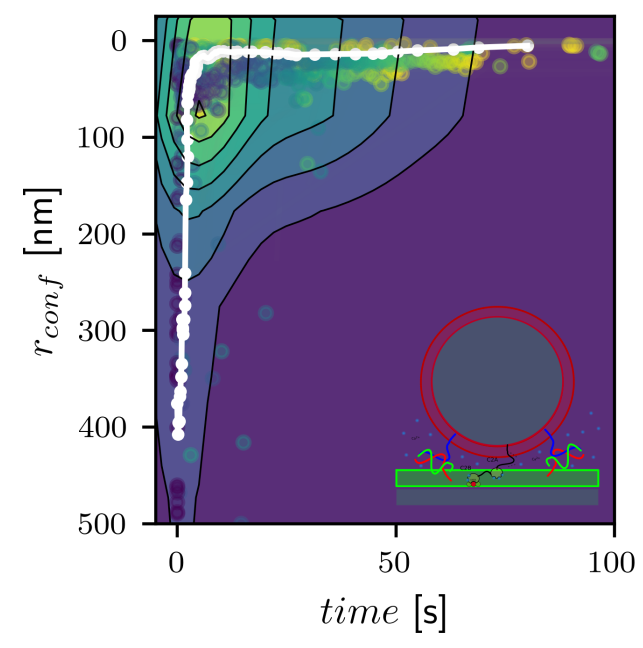

(d) Influence of Syt-1 and calcium

Figure 6.18: KDE overlayed Scatterplots of membrane coated bead trajectories (DOPC:POPE:Cholesterol:TexasRed-DPPE doped either with (a) syb1-116: $\mathrm{p} / 1=$ 1:500; $(\mathrm{b}+\mathrm{c})$ syb1-116: $\mathrm{p} / 1=1: 500$ and Syt-1: $\mathrm{p} / 1=1: 1000)$ after adhesion to SSM (DOPC:POPE:POPS:Cholesterol:PI(4,5)P $\mathrm{P}_{2}$ :Atto488-DPPE : (48:19:10:20:2:1), $\Delta \mathrm{N}: \mathrm{p} / 1=$ 1:500) recorded with HVPT. Derived is a median trajectory of membrane fusion from the confinement radius of the bead's movement over a period of $100 \mathrm{~s}$; shown in white. 
Last, we summarize the results from the tethered particle motion experiments and the influence of $\mathrm{PI}(4,5) \mathrm{P}_{2}$ on the SNARE-mediated membrane fusion for calcium-mediated and synaptotagmin-triggered exocytosis. The results are shown in a bar plot with a median estimator and are grouped by target bilayer composition, respectively (Fig. 6.19).

We observe that an increasing negative charge of the target membranes on the planar support leads to substantially smaller confinement radii and, therefore, closer contact between the opposing membranes the presence of $\mathrm{Ca}^{+2}$ strongly enhances this effect. In the absence of calcium and with the reconstitution of synaptotagmin, we observe faster and more stable confinement. Confinement radii are larger than on pure bilayer in the presence of calcium. Thus more mobility and more stable binding than in the control experiments in the presence of calcium were observed. This is why we conclude that synaptotagmin induces a charge-mediated binding and that it additionally binds to the membrane and tethers as soon as $\mathrm{PI}(4,5) \mathrm{P}_{2}$ is available.

If you consider the median radius of movement for the SNARE experiments on $\mathrm{PI}(4,5) \mathrm{P}_{2}$ $2 \%$ bilayers, this is greater than, for example, $\mathrm{PI}(4,5) \mathrm{P}_{2} 5 \%$. In this illustration, it must be ensured that all segments are included to form the distribution. Hence, it appears that SNAREs without co-reconstitution dock more densely in the distribution. However, this is not the case. Instead, states are mixed up. SNAREs show the most efficient fusion. There were hardly any previous states, such as the docking of synaptotagmin. SNAREs fuse to the target membrane almost immediately upon contact. Co-reconstituted SNAREs initially dock reasonably quickly to the membrane via synaptotagmin before fusion is triggered. Some remain in a waiting position if no calcium ions are available. This creates larger confinement radii. For this reason, we extracted the lifetime between docking and fusion and considered its fraction concerning the total number of segments (Fig. 6.21 and 6.27. S0 and S6). The observation of this lifetime does indeed show the influence of synaptotagmin on the SNARE-mediated membrane fusion. Not only is the docking affinity increased by the presence of synaptotagmin, but the time to fusion is also reduced (Fig. 6.20). We believe this course can be resolved in greater detail with methods such as optical tweezers; hence during an action potential, $\mathrm{Ca}^{2+}$ entering a presynaptic terminal triggers synaptic vesicle exocytosis and neurotransmitter release in less than a millisecond. With this approach, we produce results which, on the one hand, reproduce individual events and individual trajectories from vesicle experiments. On the other hand, we are able to produce results with good statistics comparable to bulk assays. 


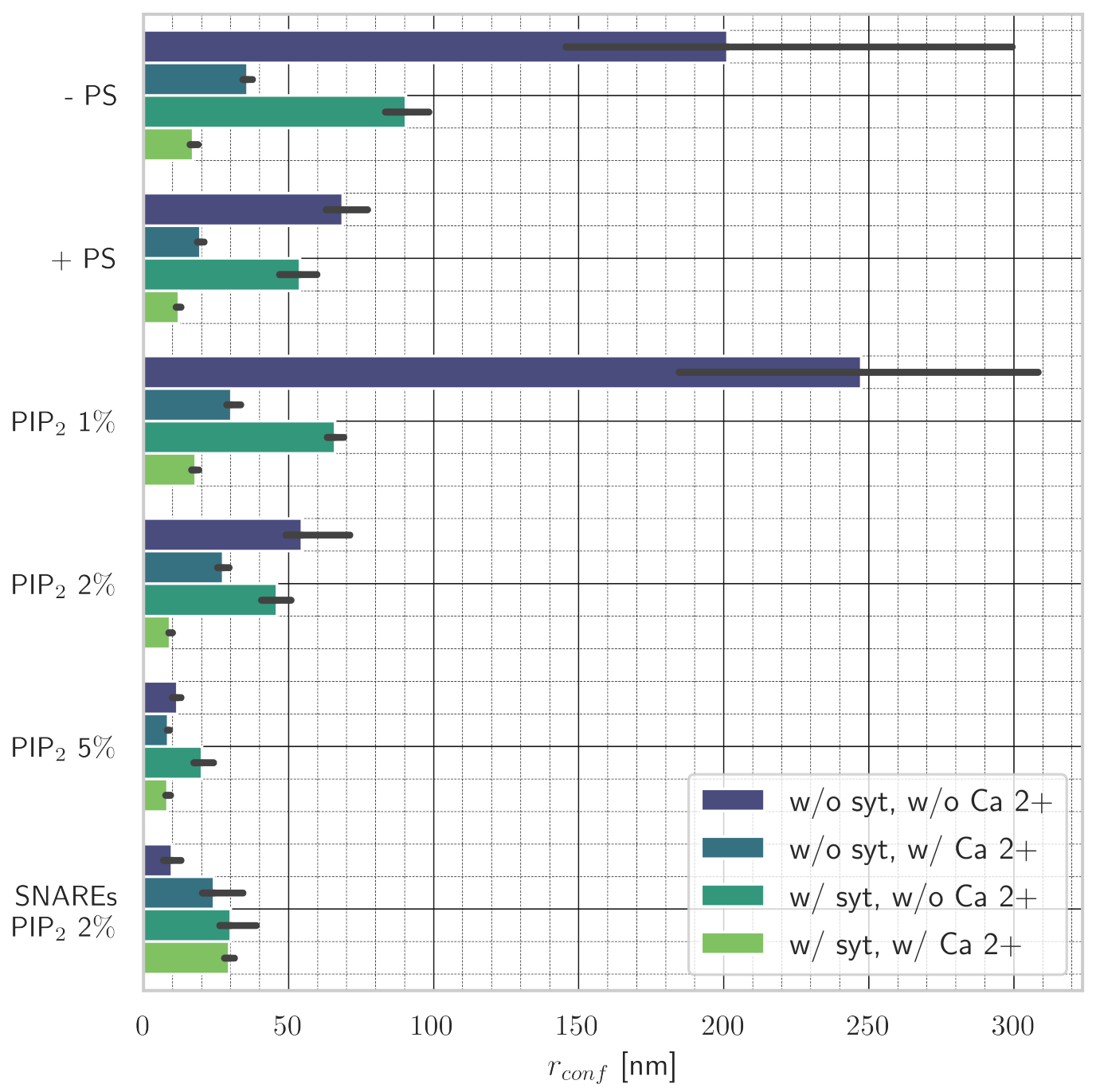

Figure 6.19: Influence of $\mathrm{PI}(4,5) \mathrm{P}_{2}$ on the SNARE mediated membrane fusion for calcium-mediated and synaptotagmin-triggered exocytosis. Median estimator of confinement radii $r_{\text {conf }}$ from TPM experiments against different membranes in the presence and absence of Syt-1, calcium ions, and SNARE complex $($ syb1-116 $+\Delta \mathrm{N})$. Results are grouped by composition of target membranes: (DOPC:POPE:POPS:Chol:PI(4,5)P ${ }_{2}$ :Atto488-DPPE: 50:29:0:20:0:1 (-PS), 50:19:10:20:0:1 (PS), 50-n:19:10:20:n:1 ( $\left.\left.\mathrm{PIP}_{2} n=1 \%, 2 \%, 5 \%\right)\right)$. Color-coded are presence and absence of affecting agents: Syt- 1 and $\mathrm{Ca}^{2+}$. 


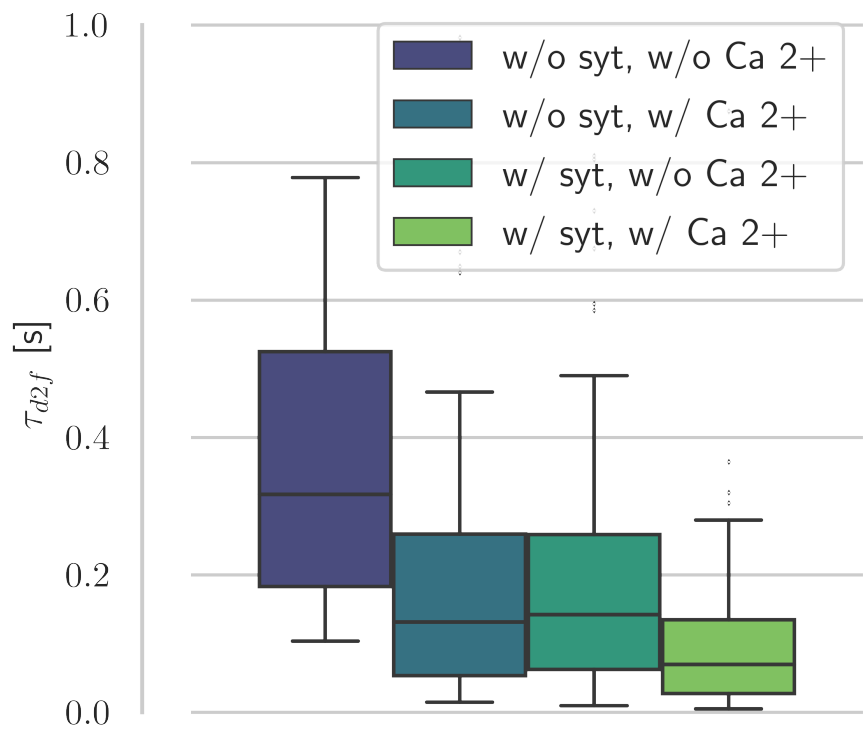

SNAREs, $\mathrm{PIP}_{2} 2 \%$

Figure 6.20: Impact of Syt-1 and calcium on the SNARE mediated membrane fusion time from docking to fusion $\tau_{\mathrm{d} 2 \mathrm{f}}$. Membrane coated bead: DOPC:POPE:Chol:TexasRed-DPPE doped either with syb1-116: $\mathrm{p} / 1=1: 500 ;$ syb1-116: $\mathrm{p} / 1=1: 500$ and Syt-1: $\mathrm{p} / 1=1: 1000$. Target membrane: DOPC:POPE:POPS:Chol:PI(4,5)P ${ }_{2}$ :Atto488-DPPE: 48:19:10:20:2:1 doped with $\Delta \mathrm{N} \mathrm{p} / 1=1: 500$. 


\section{Supporting Figures:}
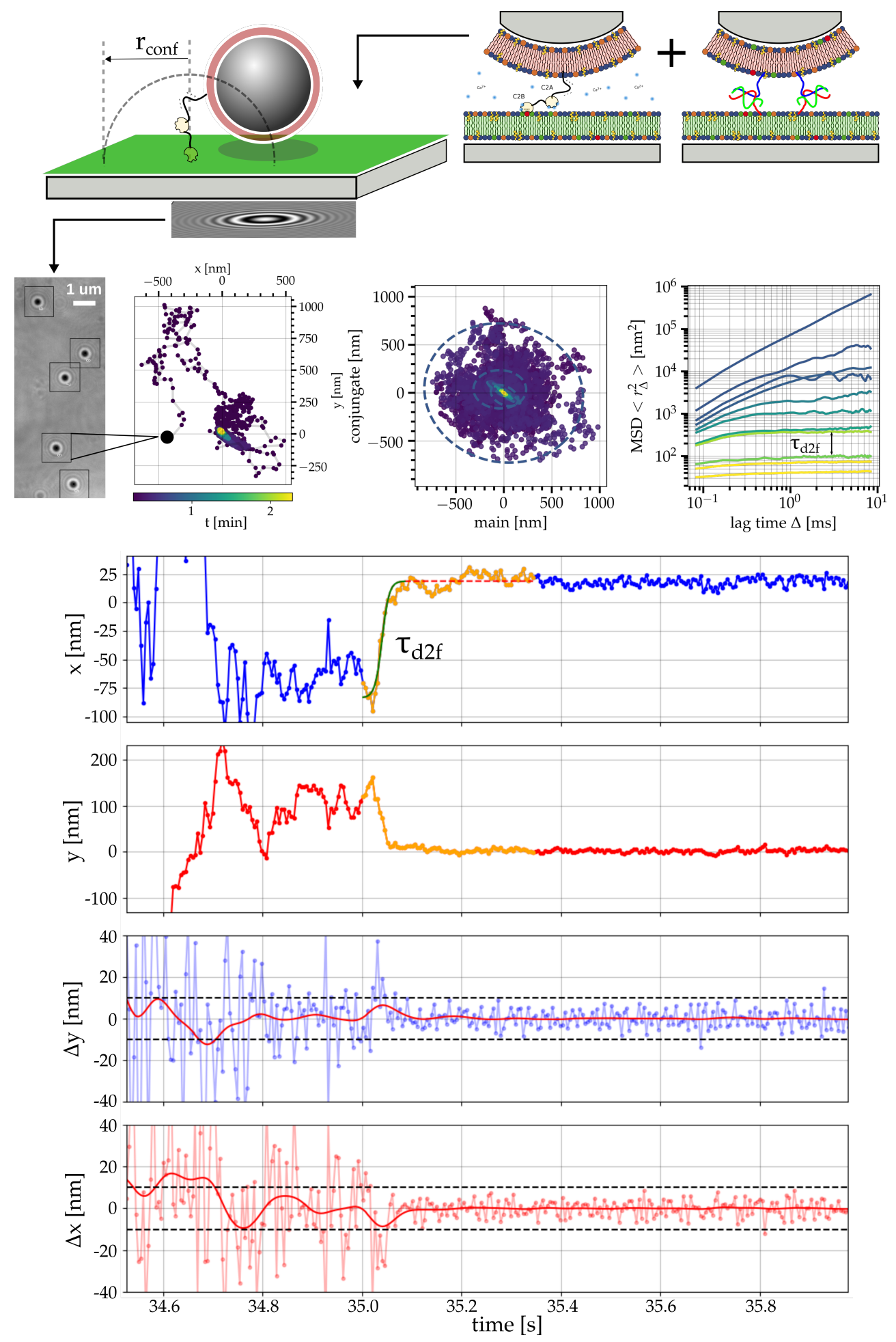

Figure 6.21: S0: The experimental approach of tethered particle motion to characterize Syt-1 aided tethering followed by syb1-116 $+\Delta \mathrm{N}$ induced membrane fusion, by analysis of bead trajectories with automated segmentation characterization by mean square displacement. The time from docking to fusion can be obtained from fitting of a generalized sigmoidal function. 

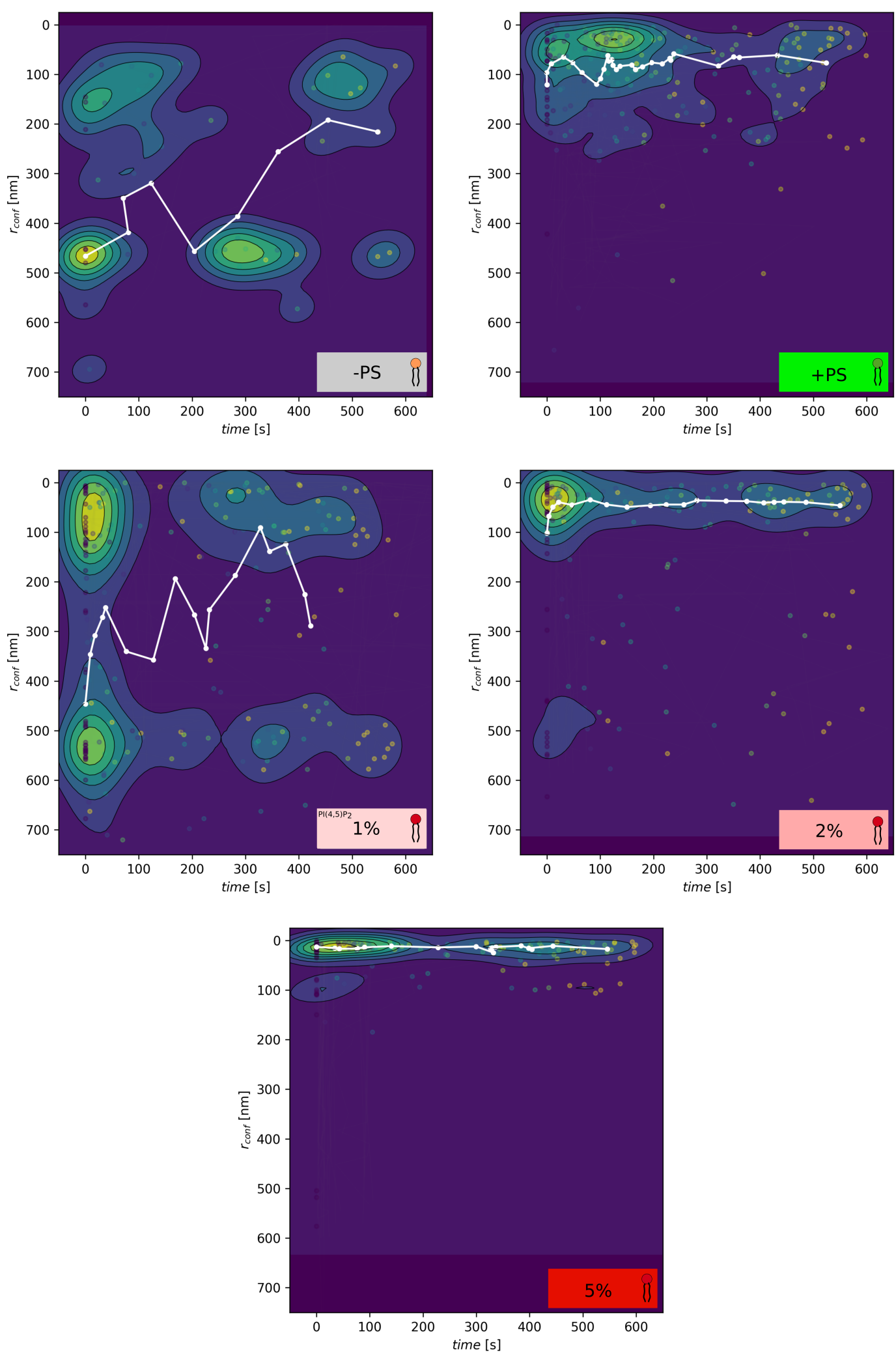

Figure 6.22: S1: Landscape construction from TPM experiments without Syt-1 and without addition of calcium recorded with HVPT at $59.8 \mathrm{~Hz}$, over a time period of $600 \mathrm{~s}$. Bilayers consisting of PC:PE:Chol:TR-DPPE; SSM varying with PS and PIP $(4,5)_{2}$ molar ratios $n$ (PC:PE:PS:Chol:PI(4,5)P 2 :Atto488-DPPE: 50:29:0:20:0:1, 50:19:10:20:0:1, 50-n:19:10:20:n:1 $n=1 \%$, $2 \%, 5 \%)$. Median trajectory is shown in white. 

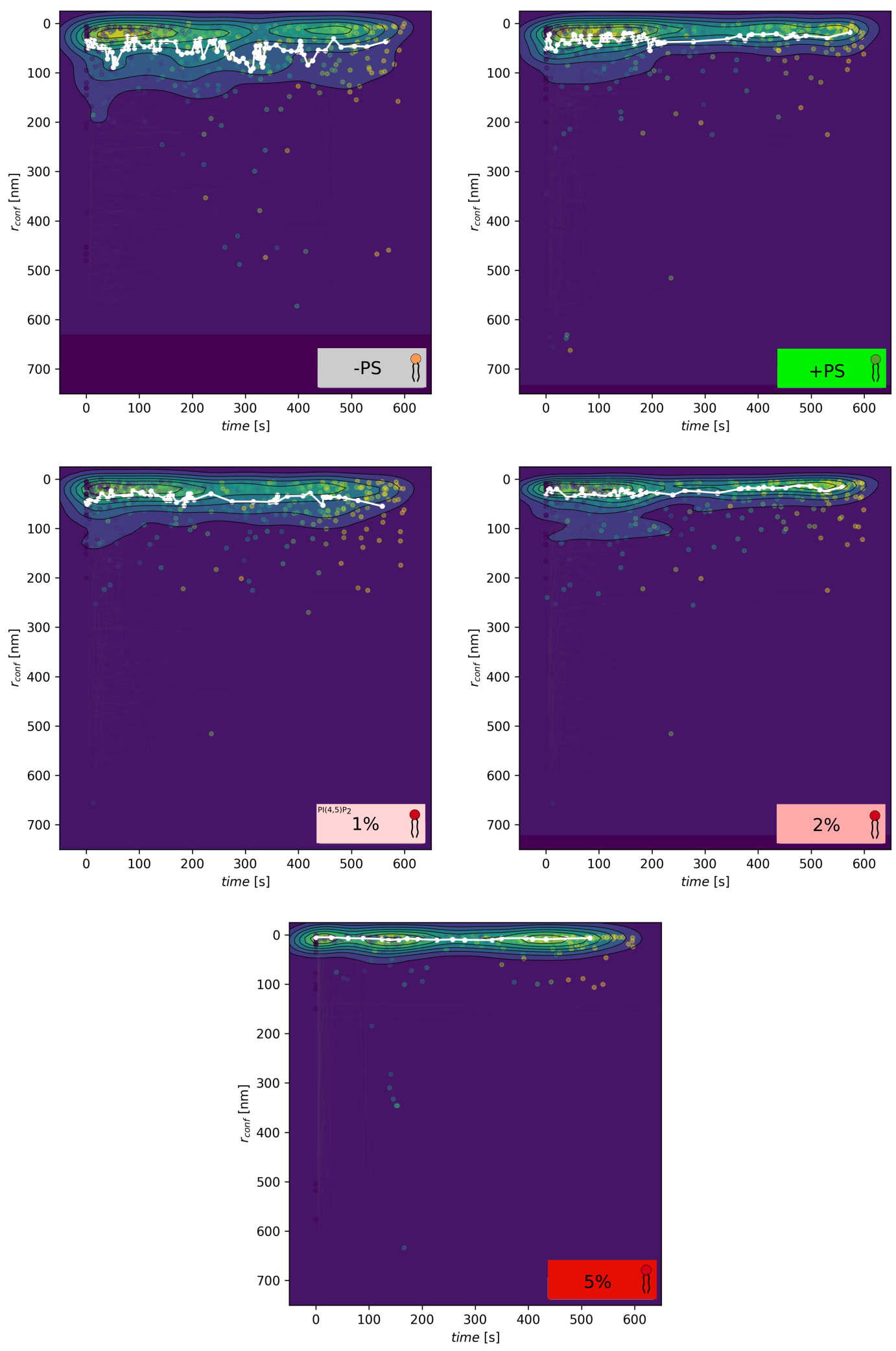

Figure 6.23: S2: Landscape construction from TPM experiments without Syt-1 and with addition of calcium recorded with HVPT at $59.8 \mathrm{~Hz}$, over a time period of $600 \mathrm{~s}$. Bilayers consisting of PC:PE:Chol:TR-DPPE; SSM varying with PS and PIP $(4,5)_{2}$ molar ratios $n$ (PC:PE:PS:Chol:PI(4,5)P 2 :Atto488-DPPE: 50:29:0:20:0:1, 50:19:10:20:0:1, 50- $n: 19: 10: 20: n: 1 n=1 \%$, $2 \%, 5 \%)$. Median trajectory is shown in white. 

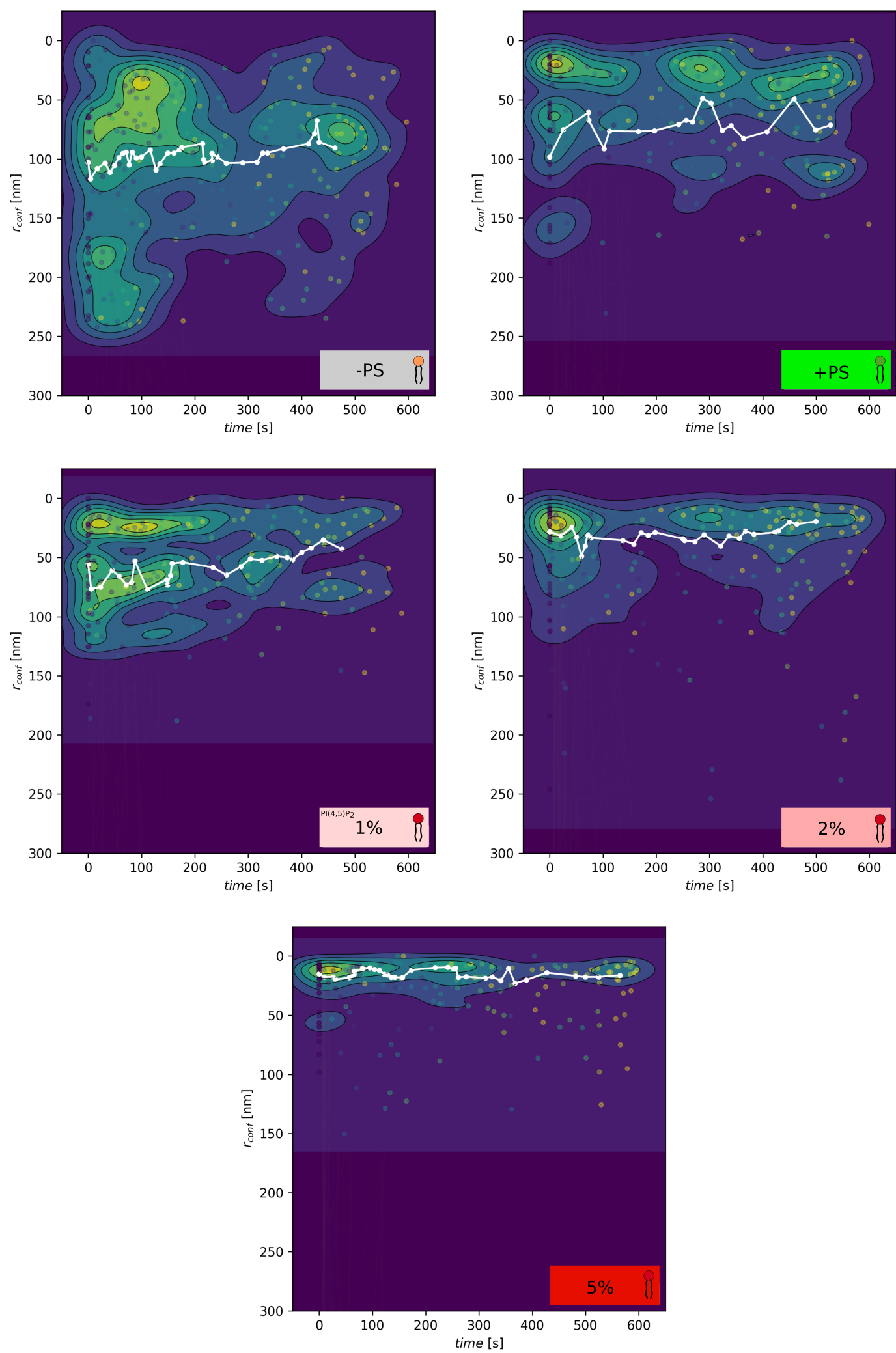

Figure 6.24: S3: Landscape construction from TPM experiments with Syt-1 and without addition of calcium recorded with HVPT at $59.8 \mathrm{~Hz}$, over a time period of $600 \mathrm{~s}$. Bilayers consisting of PC:PE:Chol:TR-DPPE; SSM varying with PS and PIP $(4,5)_{2}$ molar ratios $n$ (PC:PE:PS:Chol:PI(4,5)P 2 :Atto488-DPPE: 50:29:0:20:0:1, 50:19:10:20:0:1, 50-n:19:10:20:n:1 n=1\%, $2 \%, 5 \%)$. Median trajectory is shown in white. 

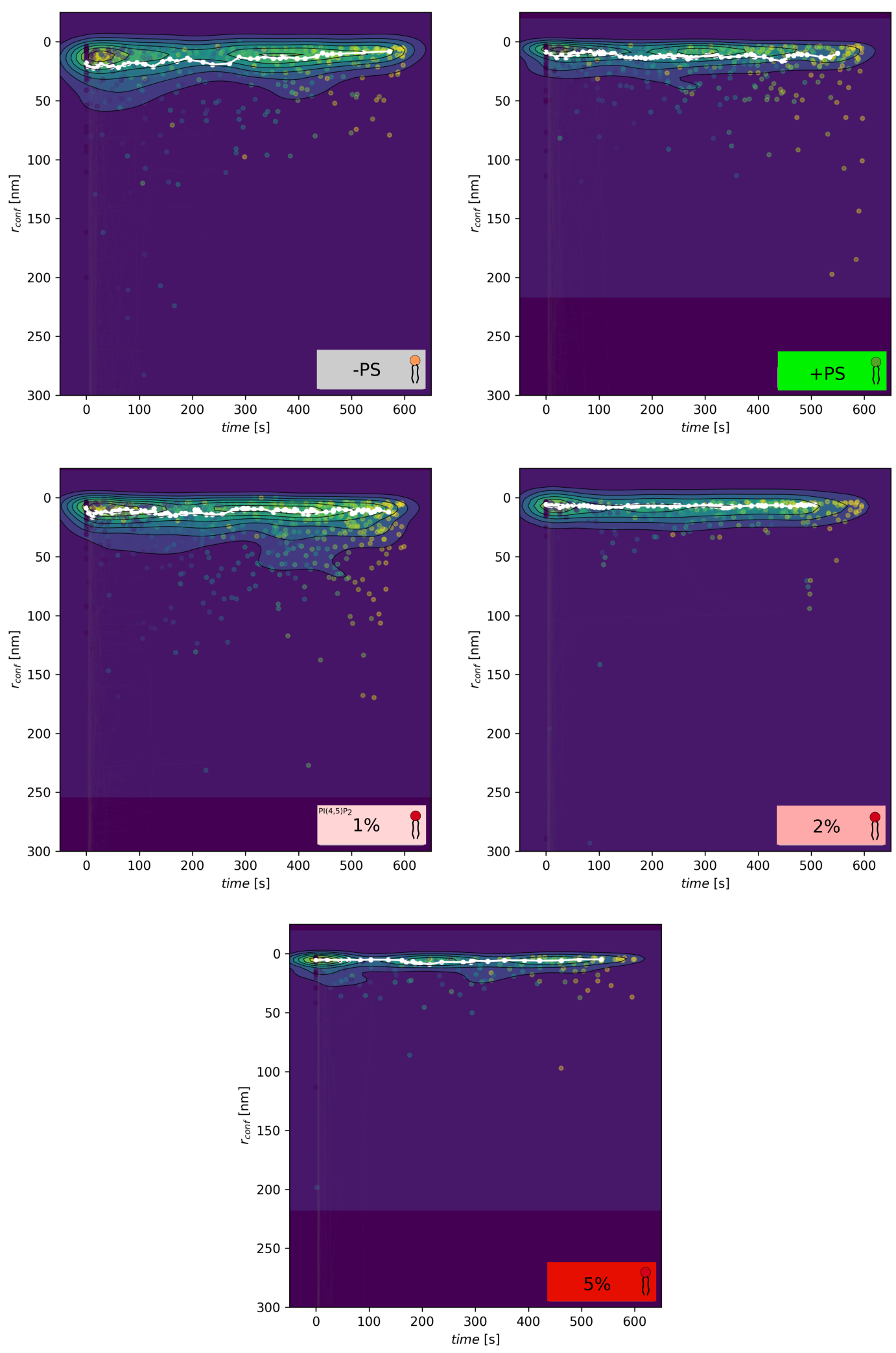

Figure 6.25: S4: Landscape construction from TPM experiments with Syt-1 and with addition of calcium recorded with HVPT at $59.8 \mathrm{~Hz}$, over a time period of $600 \mathrm{~s}$. Bilayers consisting of PC:PE:Chol:TR-DPPE; SSM varying with PS and PIP $(4,5)_{2}$ molar ratios $n$ (PC:PE:PS:Chol:PI(4,5)P 2 :Atto488-DPPE: 50:29:0:20:0:1, 50:19:10:20:0:1, 50-n:19:10:20:n:1 n=1\%, $2 \%, 5 \%)$. Median trajectory is shown in white. 


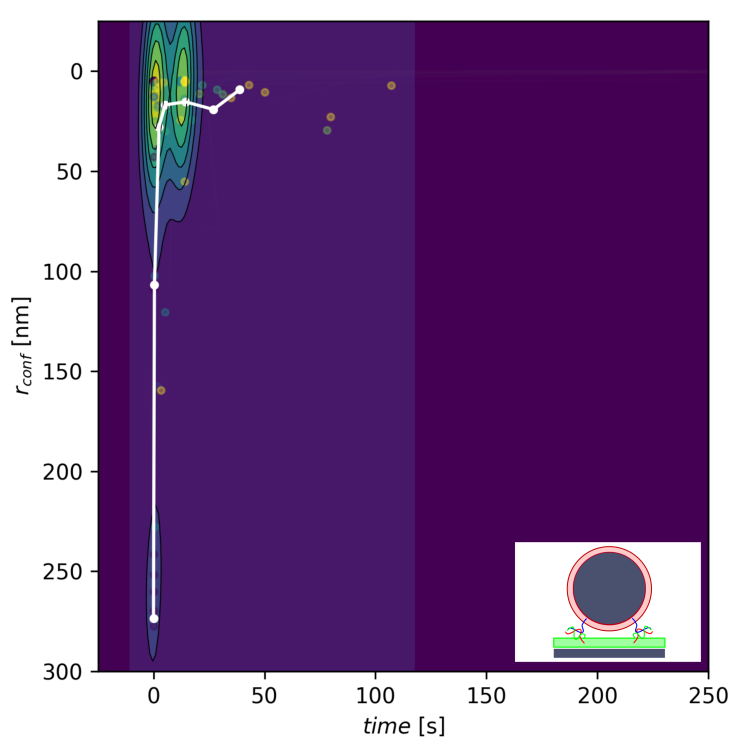

(a) SNAREs

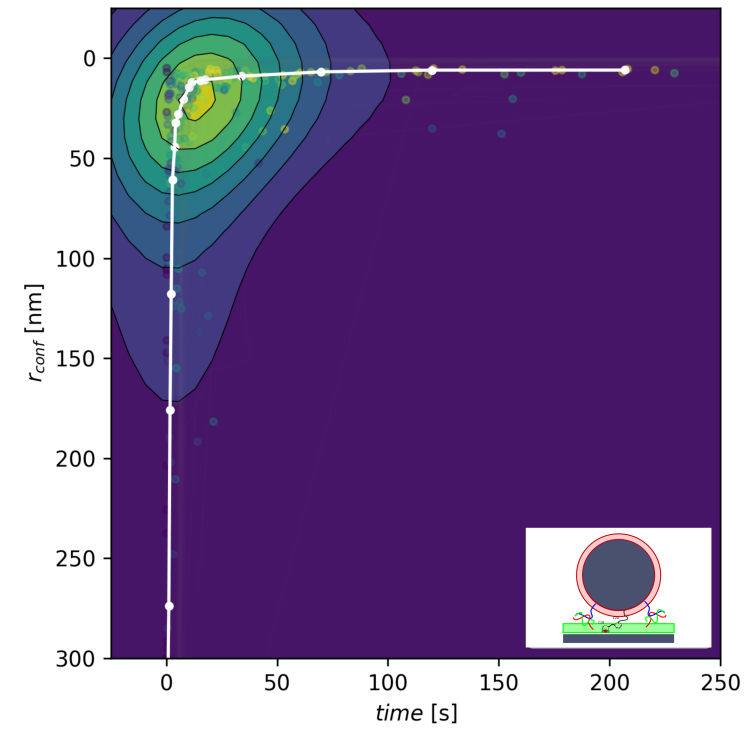

(b) Influence of f-Syt-1

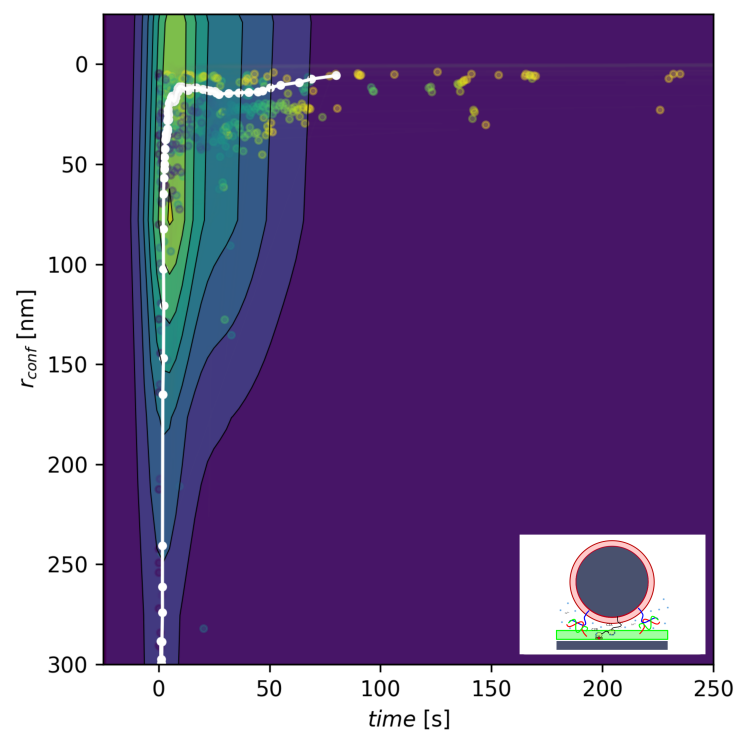

(c) Influence of f-Syt-1 and $\mathrm{CaCl}_{2}$

Figure 6.26: S5: Landscape construction of SNARE mediated membrane fusion and the influence of Syt-1 and calcium ions. Scatterplots of $r_{\text {conf }}$ as function of time from membrane coated bead trajectories (DOPC:POPE:Chol:TexasRed-DPPE doped either with syb1-116: $\mathrm{p} / 1=1: 500$; or syb1-116: $\mathrm{p} / 1=1: 500$ and Syt-1: $\mathrm{p} / 1=1: 1000)$; SSM (DOPC:POPE:POPS:Chol:PI(4,5)P 2 :Atto488-DPPE : $(48 / 19 / 10 / 20 / 2 / 1), \Delta N: p / 1=$ 1:500) recorded with HVPT. Derived is a median trajectory of membrane fusion from the confinement radius of the bead's movement over a period of $250 \mathrm{~s}$; shown in white. 


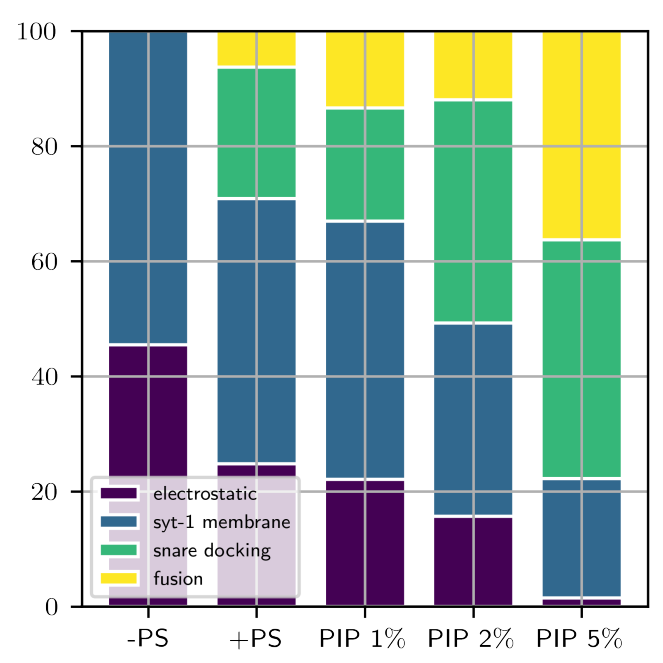

(a) Syt-1

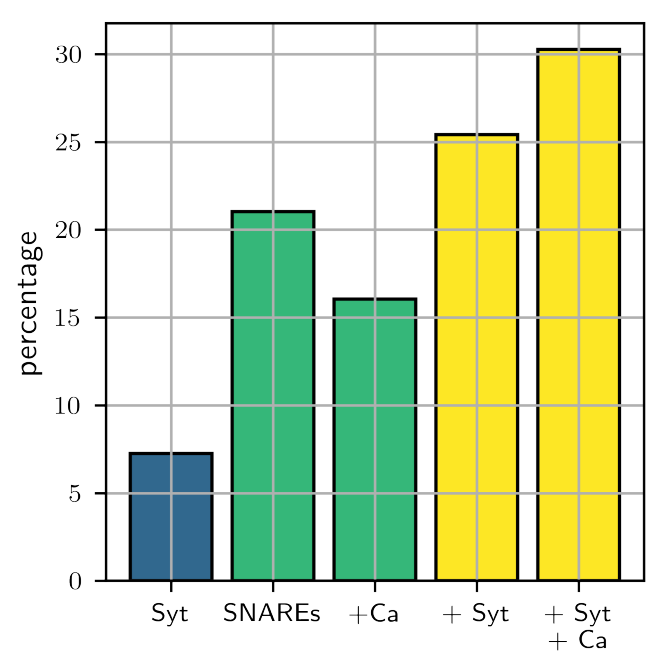

(b) SNARES

Figure 6.27: S6: Influence of $\mathrm{PI}(4,5) \mathrm{P}_{2}$ on interbilayer interactions and effects of calcium and synaptotagmin on SNARE mediated membrane fusion efficiency. (a): Shown are fractional percentages over all measured segments equivalent to electrostatic interaction, Syt-1-membrane interaction, SNARE docking and SNARE fusion derived from adhered beads. (b): Shown are fractional percentages over all measured beads only for segments capable of fusion $r_{\text {conf }}<11 \mathrm{~nm}$. 


\subsection{Conclusion}

Membrane-coated colloidal probes (CPM) combine the benefits of solid-supported membranes with defined three-dimensional geometry easy to prepare. This combination makes them a powerful model system that enables the visualization of dynamic biological processes with high throughput in TPM experiments and high temporal and spatial resolution in CPM experiments based on AFM and OT. We showed that the conversion of complex and intricate biological processes, such as SNARE mediated membrane fusion, into simple model systems can be performed using colloidal probes. With full control of lipid composition and inexpensive preparation of many probes simultaneously, efficient modeling can be carried out with the least possible stress on the proteins. In combination with optical microscopy, optical tweezers, atomic force microscopy, and colloidal probes together all provide a flexible way of studying membrane fusion with minimal reliance on fluorescent labels while being highly sensitive to its environment. These probes provide us an insight into enormously complex and regulated processes that depend on the smallest of analytes in solution.

For example, Hubrich et al. propose that the $\mathrm{Ca}^{2+}$-promoted insertion of the C2domains into the target membrane abolishes the intermediate fusion state resulting in a lack of intermediate fusion state in the presence of $\mathrm{Ca}^{2+}[10]$. We apply fundamental biophysical approaches to investigate whether the increased electrostatic interaction or interference with the membrane mechanics or, very likely, both influences membrane fusion rates. Thus, how it influences the course of the membrane merging. From TPM, we have gathered the range of motion of restricted movements. We reflected the principle from Visser et al. [?], who had demonstrated that the motion patterns of molecularly tethered particles are susceptible to the molecular system binding the particle to the substrate and the morphology near the molecular attachment point in the context of membrane fusion. In comprehensive, it is forthwith of interest to determine the exact distance between the membranes. We intuitively assume that smaller gaps between membranes lead to more friction and more hydrodynamic interaction between particles and substrate. In conventional TPM studies employing linear tethers, it is possible to establish a well-defined relation between the tether length and the magnitude of Brownian fluctuations of the bead and use this as a calibration curve. However, taken together, our data provide a mapping of the Syt-1 membrane binding energy and its role in SNARE-mediated membrane fusion. Furthermore, tethered particles can show a large variety of motion patterns. For example, double molecular tethering leads to stripe-shaped motion patterns, which we also observe. We speculate about small protrusions from the membrane tethers, cis insertion from the $\mathrm{C} 2$ domain into membranes, and a different number of Syt-tether or docking proteins capable of influencing 
merging. This approach can benefit from deciphering two significant contributions of membrane fusion: first, small membrane protrusions in the vicinity of the molecular bond and the number of tethers involved in tethering and ignition of membrane fusion. CPM as a combination of TPM and AFM results indicate that membranes can be closely linked with anionic phospholipids and polyanion $\mathrm{PI}(4,5) \mathrm{P}_{2}$ when Syt-1 is present and speculated trigger fusion by narrowing the gap between them. Our data shows that at this stage, full-length Syt-1 keeps the two membrane bilayers at a distance of a few nanometres, which is much shorter than the maximum length of one of the linkers is unstructured and fully stretched. This distance corresponds to that when the SNARE motifs begin to assemble and form the four-helix coil bundle, which means that Syt-1 holds the two membranes at this distance so that the fusion machine is ready for rapid initiation.

The investigations of this work with synaptotagmin have shown that it is possible to carry out colloidal probe investigations with the synaptotagmin anchored in a membrane and increase interactions between the protein and the membrane to detect. In addition to the various interactions, it also turned out that the unfolding of the C2A domain with this method and thus the force and position of the other interactions of the $\mathrm{C} 2 \mathrm{~B}$ domain with the membrane could be determined. Our approach aims to be consistent with C2A and C2B's physiological roles in mediating evoked neurotransmitter release focusing on trans interaction. But nether less, this approach can give a perspective on observations of the cooperative behavior of the C2 domains of Syt-1, which may explain the presence of multiple $\mathrm{C} 2$ domains in synaptotagmins and other C2-domain proteins. This confirmed that the Colloidal Probe model system is very well suited to detect and investigate interactions between proteins and membranes up to and including fusions.

Additional knowledge can be obtained from the deployment of external forces to discover the energy environment of membrane merging. Prominent intermediate states can be assigned precisely and quantified objectively with this toolbox of methods. Further down the road it might even be possible to reach areas of temporal resolution that can only be achieved through computer simulations. Colloidal probes have an excellent opportunity to provide further insights into the specific details of membrane fusion and its machinery, especially in the light of recent technological improvements, as high speed camera acquisition or new ways to manipulate colloidal probes are emerging. Acoustic force spectroscopy, where a standing acoustic wave is used to apply a force to microspheres, is one selected example facilitating high-throughput tests with tracking multiple colloidal probes simultaneously. Also, it is possible on the one hand to resolve individual events and, on the other hand, to reproduce bulk 
measurements using easily accessible statistics. Finally, besides the central SNARE complex, it would be of high importance to reconstitute other aspects of the fusion machinery or to introduce any kind of mutations. Furthermore, one could control the properties of the substrate by introducing additional curvature or trying to extend the control over membrane tension by using stretchable substrates like PDMS. This can answer basic questions such as why synaptic vesicle exocytosis specifically at the active zones is much quicker relative to other fusion systems, such as in endocrine cells [8, 19].

\section{Reference}

[1] Guido Bolognesi, Mark S. Friddin, Ali Salehi-Reyhani, Nathan E. Barlow, Nicholas J. Brooks, Oscar Ces, and Yuval Elani. Sculpting and fusing biomimetic vesicle networks using optical tweezers. Nature Communications, 2018. 9(1): 1-11. doi:10.1038/s41467-018-04282-w.

[2] Nils Brose, Alexander G. Petrenko, Thomas C. Sudhof, and Reinhard Jahn. Synaptotagmin: A calcium sensor on the synaptic vesicle surface. Science, 1992. 256(5059): 1021-1025. doi:10.1126/science.1589771.

[3] K. N. J. Burger and A. J. Verkleij. Membrane fusion. Experientia, 1990. 46(6): 631-644. doi:10.1007/BF01939702.

[4] Hans Jürgen Butt, Brunero Cappella, and Michael Kappl. Force measurements with the atomic force microscope: Technique, interpretation and applications. Surface Science Reports, 2005. 59(1-6): 1-152. doi:10.1016/j.surfrep.2005.08.003.

[5] Leonid V Chernomordik and Michael M Kozlov. Mechanics of membrane fusion. Nature structural $\{\mathcal{E}\}$ molecular biology, 2008. 15(7): 675-683.

[6] Aurora Dols-Perez, Victor Marin, Guillermo J. Amador, Roland Kieffer, Daniel Tam, and Marie Eve Aubin-Tam. Artificial Cell Membranes Interfaced with Optical Tweezers: A Versatile Microfluidics Platform for Nanomanipulation and Mechanical Characterization. ACS Applied Materials and Interfaces, 2019. 11(37): 33620-33627. doi:10.1021/acsami.9b09983.

[7] Clémence Gruget, Oscar Bello, Jeff Coleman, Shyam S. Krishnakumar, Eric Perez, James E. Rothman, Frederic Pincet, and Stephen H. Donaldson. Synaptotagmin-1 membrane binding is driven by the $\mathrm{C} 2 \mathrm{~B}$ domain and assisted cooperatively by the C2A domain. Scientific Reports, 2020. 10(1): 1-10. doi:10.1038/s41598-020-74923-y. 
[8] Natalia Gustavsson and Weiping Han. Calcium-sensing beyond neurotransmitters: functions of synaptotagmins in neuroendocrine and endocrine secretion. Bioscience Reports, 2009. 29(4): 245-259. doi:10.1042/BSR20090031.

[9] L. Han, B. Lui, S. Blumberg, J. Beausang, P. Nelson, and R. Phillips. Calibration of tethered particle motion experiments. arXiv, 2008: 1-16.

[10] Raphael Hubrich. Pore-spanning membranes - a versatile tool to analyze SNAREmediated single vesicle fusion. Dissertations at Georg-August-Universität Göttingen eDiss, 2018: .

[11] Jeffrey L. Hutter and John Bechhoefer. Calibration of atomic-force microscope tips. Review of Scientific Instruments, 1993. 64(7): 1868-1873. doi:10.1063/1.1143970.

[12] Reinhard Jahn and Richard H. Scheller. SNAREs - engines for membrane fusion. Nature Reviews Molecular Cell Biology, 2006. 7(9): 631-643. doi:10.1038/nrm2002.

[13] Douwe Kamsma, Ramon Creyghton, Gerrit Sitters, Gijs J.L. Wuite, and Erwin J.G. Peterman. Tuning the Music: Acoustic Force Spectroscopy (AFS) 2.0. Methods, 2016. 105: 26-33. doi:10.1016/j.ymeth.2016.05.002.

[14] Andrea Keidel, Tobias F. Bartsch, and Ernst Ludwig Florin. Direct observation of intermediate states in model membrane fusion. Scientific Reports, 2016. 6(March): 1-11. doi:10.1038/srep23691.

[15] Volker Kiessling, Alex J. B. Kreutzberger, Binyong Liang, Sarah B. Nyenhuis, Patrick Seelheim, J. David Castle, David S. Cafiso, and Lukas K. Tamm. A molecular mechanism for calcium-mediated synaptotagmin-triggered exocytosis. Nature Structural \& Molecular Biology, 2018. 25(5): 139-148. doi:https: //doi.org/10.1038/s41594-018-0130-9.

[16] Heidi Koldsø, David Shorthouse, Jean Hélie, and Mark S.P. Sansom. Lipid Clustering Correlates with Membrane Curvature as Revealed by Molecular Simulations of Complex Lipid Bilayers. PLoS Computational Biology, 2014. 10(10). doi:10.1371/journal.pcbi.1003911.

[17] Alex J.B. Kreutzberger, Volker Kiessling, Binyong Liang, Patrick Seelheim, Shrutee Jakhanwal, Reinhard Jahn, J. David Castle, and Lukas K. Tamm. Reconstitution of calcium-mediated exocytosis of dense-core vesicles. Science Advances, 2017. 3(7).

[18] Chao Chen Lin, Jan Seikowski, Angel Pérez-Lara, Reinhard Jahn, Claudia Höbartner, and Peter Jomo Walla. Control of membrane gaps by synaptotagmin-Ca $2+$ 
measured with a novel membrane distance ruler. Nature Communications, 2014. 5: 1-7. doi:10.1038/ncomms6859.

[19] Manfred Lindau and Wolfhard Almers. Structure and function of fusion pores in exocytosis and ectoplasmic membrane fusion. Current Opinion in Cell Biology, 1995. 7(4): 509-517. doi:https://doi.org/10.1016/0955-0674(95)80007-7.

[20] J. Paul Luzio, Sally R. Gray, and Nicholas A. Bright. Endosome-lysosome fusion. Biochemical Society Transactions, 2010. 38(6): 1413-1416. doi:10.1042/BST0381413.

[21] V. S. Markin, M. M. Kozlov, and V. L. Borovjagin. On the theory of membrane fusion. The stalk mechanism. General Physiology and Biophysics, 1984. 3(5): 361-377.

[22] Vladislav S. Markin and Joseph P. Albanesi. Membrane fusion: Stalk model revisited. Biophysical Journal, 2002. 82(2): 693-712. doi:10.1016/S0006-3495(02) 75432-5.

[23] T F J Martin. Prime movers of synaptic vesicle exocytosis. Neuron, 2002. 34(1): 9-12.

[24] Thomas F.J. Martin. Role of pi $(4,5) \mathrm{p} 2$ in vesicle exocytosis and membrane fusion. Subcellular Biochemistry, 2015. 59: 111-130.

[25] Dragomir Milovanovic, Alf Honigmann, Seiichi Koike, Fabian Göttfert, Gesa Pähler, Meike Junius, Stefan Müllar, Ulf DIederichsen, Andreas Janshoff, Helmut Grubmüller, Herre J. Risselada, Christian Eggeling, Stefan W. Hell, Geert Van Den Bogaart, and Reinhard Jahn. Hydrophobic mismatch sorts SNARE proteins into distinct membrane domains. Nature Communications, 2015. 6. doi:10.1038/ ncomms6984.

[26] Frauke Mücksch, Mevlut Citir, Christian Lüchtenborg, Bärbel Glass, Alexis Traynor-Kaplan, Carsten Schultz, Britta Brügger, and Hans Georg Kräusslich. Quantification of phosphoinositides reveals strong enrichment of PIP2 in HIV-1 compared to producer cell membranes. Scientific Reports, 2019. 9(1): 1-13. doi: 10.1038/s41598-019-53939-z.

[27] KC Keir C Neuman and Attila Nagy. Single-molecule force spectroscopy: optical tweezers, magnetic tweezers and atomic force microscopy. Nature methods, 2008. 5(6): 491-505. doi:10.1038/nmeth.1218.Single-molecule.

[28] Sarah B. Nyenhuis, Anusa Thapa, and David S. Cafiso. Phosphatidylinositol 4,5 
bisphosphate controls the cis and trans interactions of synaptotagmin 1. Biophysical Journal, 2019. doi:10.1016/j.bpj.2019.06.016.

[29] Marieelen Oelkers, Hannes Witt, Partho Halder, Reinhard Jahn, and Andreas Janshoff. SNARE-mediated membrane fusion trajectories derived from forceclamp experiments. Proceedings of the National Academy of Sciences, 2016. 113(46): 13051-13056. doi:10.1073/pnas.1615885113.

[30] Yongsoo Park, Javier M. Hernandez, Geert Van Den Bogaart, Saheeb Ahmed, Matthew Holt, Dietmar Riedel, and Reinhard Jahn. Controlling synaptotagmin activity by electrostatic screening. Nature Structural and Molecular Biology, 2012. 19(10): 991-999. doi:10.1038/nsmb.2375.

[31] Ángel Pérez-Lara, Anusa Thapa, Sarah B Nyenhuis, David A Nyenhuis, Partho Halder, Michael Tietzel, Kai Tittmann, David S Cafiso, and Reinhard Jahn. PtdInsP2 and PtdSer cooperate to trap synaptotagmin-1 to the plasma membrane in the presence of calcium. eLife, 2016. 5: 1-22. doi:10.7554/elife.15886.

[32] Sanja Pöyry and Ilpo Vattulainen. Role of charged lipids in membrane structures — insight given by simulations. Biochimica et Biophysica Acta (BBA) - Biomembranes, 2016. 1858(10): 2322-2333. doi:https://doi.org/10.1016/j.bbamem.2016.03.016. Biosimulations of lipid membranes coupled to experiments.

[33] Anand Radhakrishnan, Alexander Stein, Reinhard Jahn, and Dirk Fasshauer. The $\mathrm{Ca} 2+$ affinity of synaptotagmin 1 is markedly increased by a specific interaction of its C2B domain with phosphatidylinositol 4,5-bisphosphate. Journal of Biological Chemistry, 2009. 284(38): 25749-25760. doi:10.1074/jbc.M109.042499.

[34] Alpay B. Seven, Kyle D. Brewer, Liang Shi, Qiu Xing Jiang, and Josep Rizo. Prevalent mechanism of membrane bridging by synaptotagmin-1. Proceedings of the National Academy of Sciences of the United States of America, 2013. 110(34): 3243-3252. doi:10.1073/pnas.1310327110.

[35] Raya Sorkin, Margherita Marchetti, Emma Logtenberg, Melissa Piontek, Emma Kerklingh, Guy Brand, Rashmi Voleti, Josep Rizo, Wouter H. Roos, Alexander J. Groffen, and Gijs J. L. Wuite. Synaptotagmin-1 and Doc2b exhibit distinct membrane remodeling mechanisms. bioRxiv, 2019: 538405. doi:10.1101/538405.

[36] Thomas C Südhof. The Synaptic Vesicle Cycle. Annual Review of Neuroscience, 2004. 27(1): 509-547. 
[37] Thomas C. Südhof. A molecular machine for neurotransmitter release: Synaptotagmin and beyond. Nature Medicine, 2013. 19(10): 1227-1231. doi:10.1038/nm.3338.

[38] R. Bryan Sutton, Bazbek A. Davletov, Albert M. Berghuis, Thomas C. Sudhof, and Stephen R. Sprang. Structure of the first C2 domain of synaptotagmin I: A novel Ca2+/phospholipid-binding fold. Cell, 1995. 80(6): 929-938. doi:10.1016/ 0092-8674(95)90296-1.

[39] Joseph A. Szule, Jae Hoon Jung, and Uel J. McMahan. The structure and function of \&\#x2018;active zone material\&\#x2019; at synapses. Philosophical Transactions of the Royal Society B: Biological Sciences, 2015. 370(1672): 20140189. doi:10.1098/rstb. 2014.0189.

[40] Aartjan J.W. Te Velthuis, Jacob W.J. Kerssemakers, Jan Lipfert, and Nynke H. Dekker. Quantitative guidelines for force calibration through spectral analysis of magnetic tweezers data. Biophysical Journal, 2010. 99(4): 1292-1302. doi:10.1016/j. bpj.2010.06.008.

[41] Josep Ubach, Xiangyang Zhang, Xuguang Shao, Thomas C. Südhof, and Josep Rizo. Ca2+ binding to synaptotagmin: how many ca2+ ions bind to the tip of a c2-domain? The EMBO Journal, 1998. 17(14): 3921-3930. doi:https://doi.org/10. 1093/emboj/17.14.3921.

[42] Geert Van Den Bogaart, Karsten Meyenberg, H. Jelger Risselada, Hayder Amin, Katrin I. Willig, Barbara E. Hubrich, Markus Dier, Stefan W. Hell, Helmut Grubmüller, Ulf Diederichsen, and Reinhard Jahn. Membrane protein sequestering by ionic protein-lipid interactions. Nature, 2011. 479(7374): 552-555. doi: $10.1038 /$ nature10545.

[43] Geert van den Bogaart, Karsten Meyenberg, Herre Jelger Risselada, Hayder Amin, Katrin I. Willig, Barbara E. Hubrich, Markus Dier, Stefan W. Hell, Helmut Grubmüller, Ulf Diederichsen, and Reinhard Jahn. Membrane protein sequestering by ionic protein-lipid interactions. Nature, 2011. 479: 552 - 555.

[44] Hannes Witt and Andreas Janshoff. Using Force Spectroscopy to Probe Coiled-Coil Assembly and Membrane Fusion. Springer New York, New York, NY. ISBN 978-14939-8760-3, 2018. 145-159. doi:10.1007/978-1-4939-8760-3_8.

[45] Sylvain Zorman, Aleksander A Rebane, Lu Ma, Guangcan Yang, Matthew A Molski, Jeff Coleman, Frederic Pincet, James E Rothman, and Yongli Zhang. Com- 
mon intermediates and kinetics, but different energetics, in the assembly of SNARE proteins. eLife, 2014. 3. doi:10.7554/eLife.03348.001. 


\section{APPENDIX}

Here we present a List of Figures with shortened captions, a List of Abbreveations, Curriculum Vitae and lastly the Acknowledgement. 


\section{LIST OF FIGURES}

1.1 Schematic Illustration of Complex Systems $\ldots . . . . . . . . . .22$

1.2 Schematic Illustration of an Eukaryotic Cell and its Organelles. . . . . . . 3

1.3 Schematic Illustration of Common Phospholipid Structures and Geome-

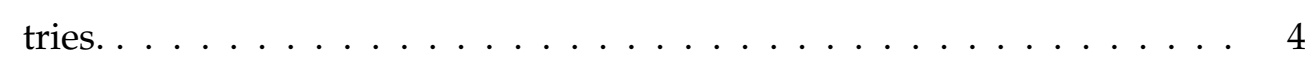

1.4 Illustration of formed Four-Helix Coiled Coil SNARE-Complexes: . . . . 6

1.5 Schematic Illustration of a Proposed Model of Tethering Induced by the C2-domains of Syt-1. . . . . . . . . . . . . . . . 6

1.6 Schematic Illustration of the Nuclear Pore Complex Structure: . . . . . . 8

2.1 Schematic Illustration of Experimental Setups to realize Colloidal Probe Force Spectroscopy: AFM, OT, and HVPT . . . . . . . . . . . . . . . 15

2.2 Experimental Setup to realize Colloidal Probe Force Spectroscopy by AFM 16

2.3 Experimental Approach for the Mechanical Evaluation of 2-Dimensional Spatial Indentation Experiments . . . . . . . . . . . . . . 17

2.4 Schematic Illustration of Indentation Experiment from AFM: Force vs.

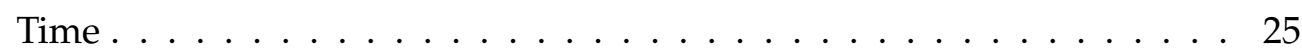

2.5 Schematic Illustration of an Indentation Experiment of a Poroelastic Material with a Spherical AFM-Tip. . . . . . . . . . . . . . . . . 26

2.6 Schematic Illustration of Experimental Setups to realize Colloidal Probe Force Spectroscopy by Optical Trapping (OT) . . . . . . . . . . . . . . . . 30

2.7 Comparison of Different Types of Illuminations: Bright-Field, Fluorescent Light, and Coherent Light Illumination. . . . . . . . . . . . . . 33

2.8 Holographic Particle Pattern. . . . . . . . . . . . . . . . . 34

2.9 Schematic Illustration of HVPT Setup to realize Colloidal Probe Force Spectroscopy by Holographic Video Particle Tracking . . . . . . . . . . 35

2.10 Schematic Illustration of a Tethered Particle Motion Experiment. . . . . . 36

2.11 Illustration of a Tethered Particle Motion Experiment and its Data Analysis 38

2.12 Different States of Particle Motion and their Categorization by Mean Square Displacement. . . . . . . . . . . . . . . . . . . . . . . . 39 
2.13 Schematic Workflow for the Analysis of a Tethered Particle Motion Experiment. . . . . . . . . . . . . . . . . 40

2.14 Application of PCA to the Analysis of Particle Motion. . . . . . . . . 42

2.15 Illustration of a Segmented Tethered Particle Motion Experiment. . . . . 43

3.1 Structural Formula and Absorption or Emission Spectrum from ATTO390, ATTO488 and Texas Red $\left.{ }^{\mathbb{B}}\right]$. . . . . . . . . . . . . . . . . . 61

3.2 Comparison of the Emission Spectra of the Fluorescent Des used . . . 62

3.3 Membrane Bilayer Patches on PDMS. . . . . . . . . . . . . . . . . . . 64

3.4 Vertical Bilayer Support for OT-Experiments. . . . . . . . . . . . . . . . . 64

3.5 Fluorescence Excitation of TexasRed labeled membrane-coated Glass Spheres Sedimenting through a Pipette Column. . . . . . . . . . . . . . 67

3.6 Fluorescence microscope image of ATTO 488 DOPE labeled membrane-

\begin{tabular}{|c|}
\hline coated glass spheres (left) with a particle size of $4.7 \mu \mathrm{m}$, with a corre- \\
\hline
\end{tabular}

sponding line profile (right). . . . . . . . . . . . . . . 68

3.7 Jablonski diagram . . . . . . . . . . . . . . . . . . . . . . . . . . . 69

3.8 Exemplary Representation of a FRAP Experiment. . . . . . . . . . . . . . 71

3.9 Exemplary Fluorescence Images of Target SSMs prepared on a Glass Sur-

\begin{tabular}{|c|}
\hline face to Study the Influence of PIP $(4,5)_{2}$ on SNARE mediated membrane \\
\hline
\end{tabular}

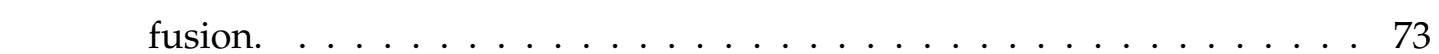

3.10 Images of a Borosilicate Glass Microsphere with a Diameter of $15 \mu \mathrm{m}$ glued to a tip-less Cantilever. . . . . . . . . . . . . . . . . 74

3.11 Phase Contrast Images of C2C12 Cells at Different Stages of Differentiation 75

4.1 Schematic Illustration of Liquid-Liquid Phase-Separation into Assemb\begin{tabular}{|l|}
\hline lies of Protein Droplets and the Partitioning of $r$ NTR2 into MacNup98A \\
\hline
\end{tabular} FG-Particle. . . . . . . . . . . . . . . . . . 82

4.2 Mechanical Approach by AFM with Colloidal Probe Force Spectroscopy. 87

4.3 Mechanical Response upon Indentation of FG-Hydrogel Particle with a Diameter of $20 \mu \mathrm{m} . \quad \ldots \ldots \ldots \ldots \ldots$. . . . . . . . . . 88

4.4 AFM-based Elasticity Measurements carried out on FG-Hydrogel Droplets. 91

$4.5 \quad$ AFM-based Poro-Elasticity Measurements and the Influence of NTR (NTF2) carried out on FG-Hydrogel Droplets. . . . . . . . . . . . . . . . . 94

4.6 Effect of NTF2 on the Poroelastic Diffusion Coefficient and the Connected Hydrogel Pore Size. . . . . . . . . . . . . . . . . . . . . 96

4.7 Comparison of the Effect of NTF2 on the Hydrogel Pore Size for two

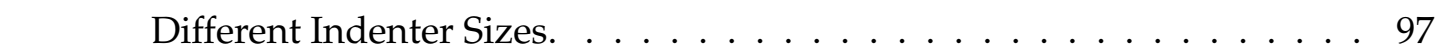

4.8 Influence of NTR on Bulk and Single Filament Rupture Events obtained from AFM Retraction Experiments. . . . . . . . . . . . . . . . . 100 
4.9 Determination of Mesh Size from Bulk Rupture Events and Discrimination from Single Filament Rupture Events. . . . . . . . . . . . . . . . . 102

4.10 S1: AFM-based Poro-Elasticity Measurements and the Influence of NTR (NTF2) carried out on FG-Hydrogel Droplets. . . . . . . . . . . . . . . . 104

4.11 S2: AFM-based Poro-Elasticity Measurements and the Influence of NTR (NTF2) carried out on FG-Hydrogel Droplets. . . . . . . . . . . . . . . 105

4.12 S3: AFM-based Poro-Elasticity Measurements and the Influence of NTR (NTF2) carried out on FG-Hydrogel Droplets. . . . . . . . . . . . . . . 106

4.13 S4: Worm-Like Chain (WLC) Analysis of Nup98 SMFS Data from Force Extension of Presumptive Single Filaments. . . . . . . . . . . . . . . 107

5.1 Phase Contrast and Fluorescence Images of C2C12 on Collagen-Coated Petri Dishes. . . . . . . . . . . . . . . . . . . . . . . . . . . . . . 118

5.2 C2C12 Myogenic Differentiation Process. . . . . . . . . . . . . . . . . . 119

5.3 Mechanical Properties of C2C12 Cells from Indentation Experiments. . . 123

5.4 Mechanical Properties of C2C12 Cells from Retraction Experiments. . . . 124

5.5 Membrane-Coated Beads-Assay in a 2D Assembly to Elucidate the Effect Ecto-Domain Myomerger on the Mechanism of Membrane Fusion. . . . 127

6.1 Schematic Illustration of Common Membrane Topology during Membrane Fusion Pathway. . . . . . . . . . . . . . . . . . . . 134

6.2 Schematic Illustration of Colloidal Probes as Membrane Fusion Assay in the Presence of SNAREs. . . . . . . . . . . . . . . . . . . . 135

6.3 Illustration of the AFM Setup equipped with the Colloidal Probes. . . . . 137

6.4 Overview of Membrane Coated Colloidal Probes Force Spectroscopy by Optical Tweezer. A: Schematic representation of the setup. The NanoTracker 2 optical tweezer (JPK Instruments, Berlin, Germany) build on an inverted Zeiss microscope equipped with a $5 \mathrm{~W} \lambda=1064 \mathrm{~nm}$ LASER and a water immersion objective (63x, C-Apochromat, NA = 1.2, Carl Zeiss, Jena, Germany). B: Spring constants of membrane coated silica beads as a function of applied LASER power for 4 different bead diameters. . . . 140

6.5 Overview of Membrane Coated Colloidal Probes Force Spectroscopy by Optical Tweezer. . . . . . . . . . . . . . . . . . . . 141

6.6 Assay to Quantify SNARE-mediated Membrane Fusion using patterned Membrane Patches on oxidized PDMS Supports. . . . . . . . . . . . . . . 143

6.7 Illustrative Result of Membrane Coated Colloidal Probes Fusion Assay by Optical Tweezer. . . . . . . . . . . . . . . . . . . . . . . . . . . 144

6.8 Binding Properties and Rupture Forces between Synaptotagmin and $\mathrm{PI}(4,5) \mathrm{P}_{2}$ containing Membranes. . . . . . . . . . . . . . . . . . . 146 
6.9 Mean Square Displacements of Tracked Membrane Coated Beads. . . . . 147

6.10 Experimental Overview of HVPT Colloidal Probe Assay to probe Mem-

brane Interactions and Membrane Fusion. . . . . . . . . . . . . . . . . . 149

6.11 Time-resolved landscape reconstruction of co-reconstitution fusion expe-

riments with Syt-1 and syb-1-116 on a membrane coated probe. . . . . . 150

6.12 S0: PCA- and MSD-Analysis of optical tweezer data with confinement

radii otbtained from fitted data before and after fusion. . . . . . . . . . 152

6.13 S1: Representative trajectory from fusion experiments with Syt-1 and

syb-1-116 on a membrane coated probe. . . . . . . . . . . . . . 153

6.14 S2: Basis of landscape reconstruction: Gaussian distributions obtained

from motion data and related to its confinement radius. Time-resolved

and color coded confinement radii and motion data over a time course

of 2 minutes, recorded with 200 frames per second. . . . . . . . . . . . 154

6.15 S3: Comparison of landscape reconstruction: KDE overlayed Scatterplots

of membrane coated bead trajectories. . . . . . . . . . . . . . . . . . 155

6.16 Schematic representation of AFM retraction sequence visualizing Syt-1

unfolding pathway and its ultimate detachment from the target membrane.159

6.17 Constructed interaction landscapes from TPM experiments in the pre-

sence and absence of $\mathrm{f}-\mathrm{Syt}-1$ and calcium ions. . . . . . . . . . . . . 163

6.18 KDE overlayed Scatterplots of membrane coated bead trajectories. . . . 164

6.19 Influence of $\mathrm{PI}(4,5) \mathrm{P}_{2}$ on the SNARE mediated membrane fusion for

calcium-mediated and synaptotagmin-triggered exocytosis. . . . . . . . 166

6.20 Impact of Syt-1 and calcium on the SNARE mediated membrane fusion

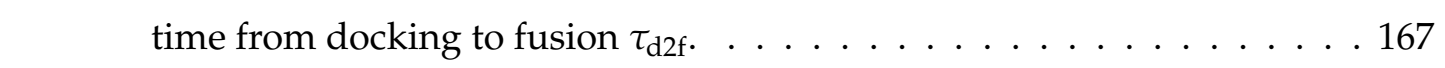

6.21 S0: The experimental approach of tethered particle motion to characterize Syt-

1 aided tethering followed by syb1-116 $+\Delta \mathrm{N}$ induced membrane fusion, by

analysis of bead trajectories with automated segmentation characterization by

\begin{tabular}{|c|}
\hline mean square displacement. The time from docking to fusion can be obtained \\
\hline
\end{tabular}

from fitting of a generalized sigmoidal function. . . . . . . . . . . . . 168

6.22 S1: Landscape construction from TPM experiments without Syt-1 and

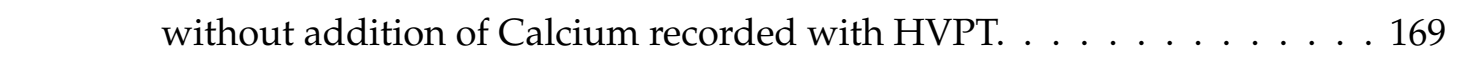

6.23 S2: Landscape construction from TPM experiments without Syt-1 and with addition of Calcium recorded with HVPT. . . . . . . . . . . . . . 170

6.24 S3: Landscape construction from TPM experiments with Syt-1 and without addition of Calcium recorded with HVPT. . . . . . . . . . . . . 171

6.25 S4: Landscape construction from TPM experiments with Syt-1 and with addition of Calcium recorded with HVPT. . . . . . . . . . . . . . . . 172 
6.26 S5: Landscape construction of SNARE mediated membrane fusion and the influence of Syt-1 and calcium ions. . . . . . . . . . . . . . . . 173

6.27 S6: Influence of $\mathrm{PI}(4,5) \mathrm{P}_{2}$ on interbilayer interactions and effects of calcium and synaptotagmin on SNARE mediated membrane fusion efficiency.174

Figure captions have been shortened, consult main text for full versions. 


\section{Abbreviations}

AFM

AFS

ALS

Arp2

ATTO 390

ATTO 488

BSA

$\mathrm{C} 2 \mathrm{AB}$

$\mathrm{C} 2 \mathrm{C} 12$

CCD

Chol

CMOS

CP

CPM

DAPI

DHPE

DI

DMEM

DNA

Doc2b
Atomic Force Microscopy

Acoustic Force Spectroscopy

Amyotrophic Lateral Sclerosis

Actin Related Protein 2/3 Complex

Fluorescence Dye ATTO 390 DOPE

Fluorescence Dye ATTO 488 DOPE

Bovine Serum Albumin

two C-terminal C2 domains - C2A and C2B of Syt-1

Immortalized mouse myoblast cell line

Charge coupled device, an electronic light sensor used in various devices including digital cameras

Cholesterin

Complementary Metal Oxide Semiconductor

Colloidal Probe

Colloidal Probe Microscopy based on AFM

4',6-diamidino-2-phenylindole, is a fluorescent stain that binds strongly to adenine-thymine-rich regions in DNA.

1,2-Dihexadecyl-sn-glycerophosphoethanolamine

Deionization Water

Dulbecco's Modified Eagle's Medium

Deoxyribonucleic Acid

Double C2-like domain-containing protein beta

DOCK1 a large ( $180 \mathrm{kDa})$ protein involved in intracellular signalling networks 
DOPC

DOPE

DOPS

DPPC

DPPE 1,2-Dipalmitoyl-sn-glycero-3-phosphoethanolamine

DTT Dithiothreitol

$\Delta \mathbf{N}$

EDTA Ethylenediaminetetraacetic Acid

EGTA Ethylene Glycol-bis( $\beta$-Aminoethyl Ether)-N,N, $\mathrm{N}^{\prime}, \mathrm{N}^{\prime}$-Tetraacetic Acid

FDC

FG

FITC

FM

FRAP

FRET

GUV

Hepes

HVPT

InvOLS

$\mathrm{KCl}$

KDE

KKKK

LLPS
Force Distance Curve

regions with a repeating pattern of two amino acids, phenylalanine $(\mathrm{F})$

and glycine $(\mathrm{G})$

Fluorescein

Force Modulation

Fluorescence Recovery After Photobleaching

Förster Resonance Energy Transfer

Giant Unilamellar Vesicle

Holographic Video Particle Tracking

Inverted Optical Lever Sensitivity

Potassium Chloride

Kernel Density Estimation

Poly Lysin Patch

Liquid Liquid Phase Separation
2-(4-(2-Hydroxyethyl)-1-piperazinyl)-ethansulfonsäure 


\begin{tabular}{|c|c|}
\hline LPC & Lysophosphatidylcholin \\
\hline LUT & Look up Table \\
\hline LUV & Large Unilamellar Vesicle \\
\hline MacNup98 & Macronuclear Nucleoporin MacNup98A \\
\hline MAD & Median Absolute Deviation \\
\hline MBP & Mannan Binding Protein \\
\hline MLCT & $\begin{array}{l}\text { Microlever AFM probes with Silicon Nitride cantilevers with Silicon } \\
\text { Nitride tips }\end{array}$ \\
\hline MLV & Multilamellar Vesicle \\
\hline MPI & Max Planck Institute \\
\hline MSD & Mean Squared Displacement \\
\hline Munc18 & Mammalian uncoordinated-18 \\
\hline n-OG & n-Octyl- $\beta$-D-Glucopyranosid \\
\hline NA & Numerical Aperture \\
\hline $\mathrm{NaCl}$ & Sodium Chloride \\
\hline NE & Nuclear Envelope \\
\hline NPC & Nuclear Pore Complex \\
\hline NTF2 & $\begin{array}{l}\text { Nuclear Transport Factor } 2 \text { is a protein that in humans is encoded by } \\
\text { the NUTF } 2 \text { gene }\end{array}$ \\
\hline NTR & Nuclear Transport Receptors \\
\hline OT & Optical Tweezer \\
\hline PBS & Phosphate Buffered Saline \\
\hline PC & Phosphatidylcholine \\
\hline PC12 & Cell line derived from a pheochromocytoma \\
\hline
\end{tabular}


PCA Principal Component Analysis

PDMS Polydimethylsiloxan

PE Phosphatidylethanolamin

PELT Pruned Exact Linear Time Algorithm

PIP2 Phosphatidylinositol-4,5-bisphosphat, PtdInsP2

PS Phosphatidylserine

PSD Position Sensitive Device

QI Quadrant Interpolation

RanGTP RAs-related Nuclear protein also known as GTP-binding nuclear protein Ran

ROI Region Of Interest

RT Room Temperature

SLFG Serin Leucin Phenylalanin Glycin

SMFS Single Molecule Force Spectroscopy

SNARE Soluble N-ethylmaleimide-sensitive-factor Attachment Receptor

SPT Single Particle Tracking

SSLM Solid Supported Liquid Membrane

SUV Small Unilamellar Vesicle

Syb-1-116 Synaptobrevin

SV Synaptic Vesicles

Syt1 calcium binding Synaptotagmin

TR $\quad$ Texas $\operatorname{Red}^{\circledR}$ DHPE

t-SNARE target-SNARE

TMD Trans Membrane Domain 
TPM

Tethered Particle Motion

v-SNARE

WLC

XCOR
vesicle-SNARE

Worm Like Chain

Cross Correlation Algorithm 